\title{
Investigation of visual motion sensitivity in the striatum of freely moving rats
}

PhD Thesis

Anett Nagy

Advisor:

Dr. Antal Berényi

Department of Physiology

Faculty of Medicine, University of Szeged

Szeged

2020 
I. Coding of self-motion-induced and self-independent visual motion in the rat dorsomedial striatum

Nagy A.J., Takeuchi Y. and Berényi A.

PLOS Biology 16 (6), e2004712. (2018)

IF: 8.386

II. Large-scale, high-density (up to 512 channels) recording of local circuits in behaving animals

Berényi A., Somogyvári Z., Nagy A.J., Roux L., Long J.D., Fujisawa S., Stark E., Leonardo A., Harris T.D., and Buzsáki G.

J. Neurophysiol. 111, 1132-1149. (2014)

IF: 2.396

A FURTHER PUBLICATION OF MINE, RELATED TO THE BASAL GANGLIA:

III. Direct projection from the visual associative cortex to the caudate nucleus in the feline brain

Nagy A.J., Berényi A., Gulya K., Norita M., Benedek G., Nagy A.

Neurosci. Lett. 503, 52-57. (2011)

IF: 2.180 


\section{TABLE OF CONTENTS}

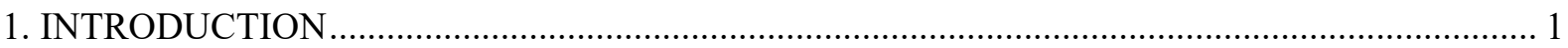

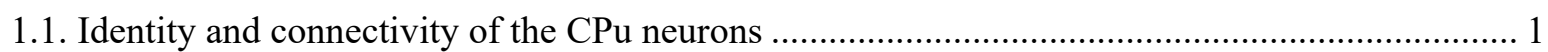

1.2. Technical challenges of the analysis of behavioral correlates of striatal neuronal networks....... 4

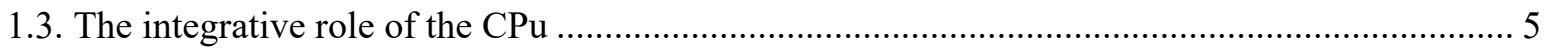

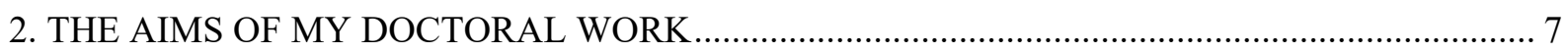

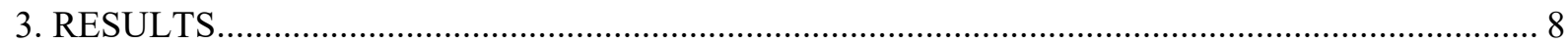

3.1 Dorsomedial striatum receives innervation from the visual cortex ........................................... 8

3.2 Large-scale extracellular recordings using multiplexed signal acquisition ................................ 11

3.3 Identity and connectivity of the recorded cells in the dorsomedial striatum ............................... 13

3.4 Striatal responses to self-independent visual stimulation .......................................................... 14

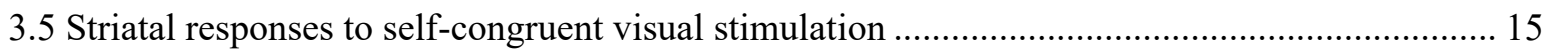

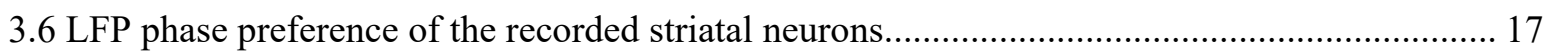

3.7 Phase-locking, space-coding and reward-related activity of the recorded neurons..................... 18

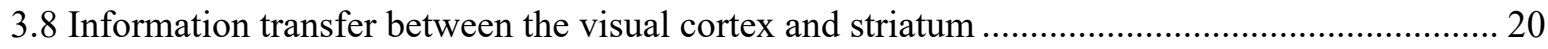

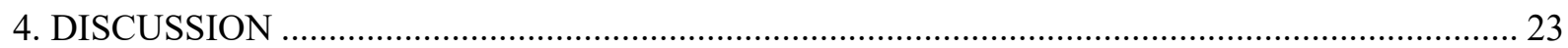

4.1. Considerations of striatal cell type identification from extracellular recordings ....................... 23

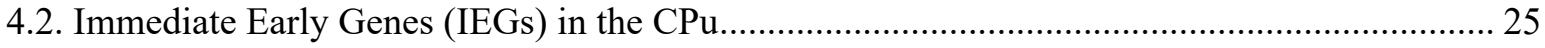

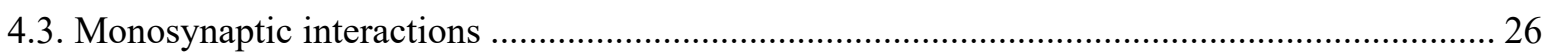

4.4. Origin and processing of the visual information in the dorsomedial striatum .......................... 27

4.5. Multimodal integration in the dorsomedial striatum - Network level oscillations ..................... 29

4.6. Multimodal integration in the dorsomedial striatum - Processing non-sensory modalities........ 31

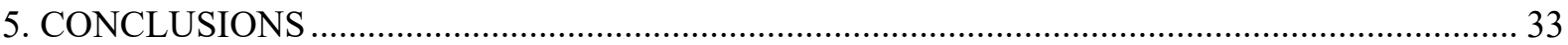

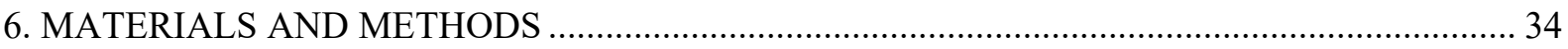

6.1. Development of multiplexing data acquisition hardware ........................................................... 34

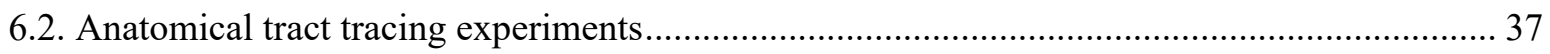

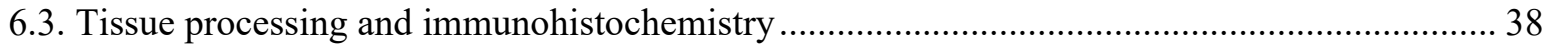

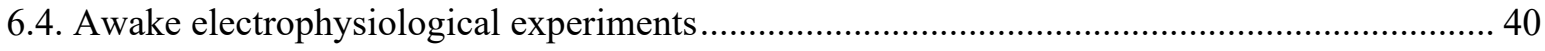

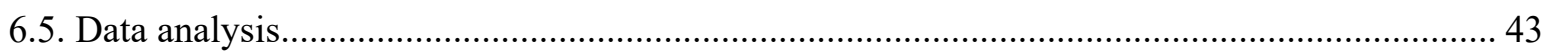

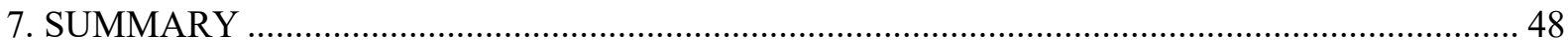

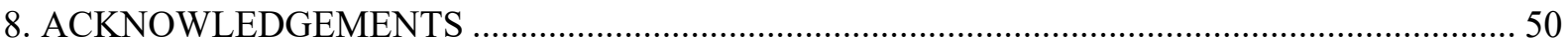

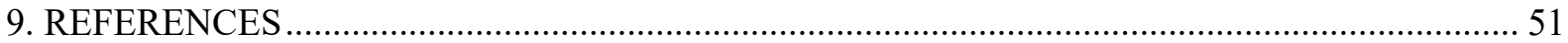




\section{LIST OF ABBREVIATIONS}

AP: anterior-posterior

a.u.: arbitrary units

aVis: active visual stimulation

BDA: biotinylated dextrane amine

BSA: bovine serum albumin

CCG: cross-correlogram

$\mathrm{CPu}$ : caudoputamen

CPU: central processing unit

CR: calretinin

DAPI: 4',6-Diamidino-2-phenylindole dihydrochloride

DAQ: digital-analog data acquisition

dB: decibel

DC: direct current

DLG: dorsolateral geniculate nucleus

DREADD: designer receptor exclusively activated by designer drugs

DV: dorsoventral

El: electrical stimulation evoked response

EM: expectation-maximization

EPSP: excitatory postsynaptic potential

FFN: fast-firing neuron

fMRI: functional magnetic resonance imaging

FR: firing rate

FSI: fast-spiking interneurons

GABA: gamma-aminobutyric acid

Hz: Hertz

IC: integrated circuit

IEGs: immediate early genes

IgG: immunoglobulin G antibody

ISI2s: interspike-intervals exceeding two seconds

L-DOPA: levodopa

LED: light-emitting diode
LFP: local field potential

LGN: lateral geniculate nucleus

LPMR: lateral-posteromedial thalamus

LTS: low-threshold spike

mCtx: motor cortex

MI: modulation index

ML: mediolateral

MSN: medium spiny neuron

NGS: normal goat serum

NOS: nitric oxide synthase

NPY: neuropeptide Y

PB: phosphate buffer

PBS: phosphate buffer saline

PBS-X: phosphate buffer saline containing

Triton X-100

PBS-XBSA: phosphate buffer saline

containing bovine serum albumin

PFA: paraformaldehyde

PFN: phasically firing neuron

PLTS: persistent low-threshold spike

Po: posterior

PSST: post-spike suppression time

PSTH: peristimulus time histogram

PV: parvalbumin

pVis: passive visual stimulation

SD: standard deviation

SNc: substantia nigra pars compacta

SO: somatostatin

SWR: sharp-wave ripple

TFN: tonically firing neuron

USB: universal serial bus

V1: primary visual cortex

V2: secondary visual cortex

vCtx: visual cortex

VLSI: very large-scale integration 


\section{INTRODUCTION}

To accommodate the ever-changing demands of survival and to maintain homeostasis, nervous systems must effectively utilize sensory information. These inputs represent the actual relation of the organism to its environment while performing goal-oriented motor activities. In order to select the behavior from the available innate or learned repertoire, which is likely to generate the highest reward value, the 'percept' must be compared to past experiences encoded in the brain as memory engrams. Although we believe that this very complex process is the holistic result of the cooperation of many circuits and regions of the brain, the striatum may still play an outstandingly important role in the sensory-motor translation. It funnels massively converging inputs from virtually all cortical areas and many subcortical structures (Hunnicutt et al., 2016) to the basal ganglia loop, and serves multiple aspects of cognition including decision-making, reward prediction and action planning (Wilson, 2014). Early concepts assumed that the striatum was purely responsible for movement-related functions, as the most common disorders, which are related directly or indirectly to the caudoputamen $(\mathrm{CPu}$, e.g., Parkinson's disease, Huntington's chorea, dyskinesia) show overwhelmingly motor deficits.

\subsection{Identity and connectivity of the CPu neurons}

Despite their extensive interconnections, the $\mathrm{CPu}$ processes information in a very distinct way from the cortex. The glutamatergic inputs originate from multiple cortical areas of both hemispheres, including sensory, motor, and prefrontal cortices. The internal circuitry of the $\mathrm{CPu}$ is shaped almost exclusively by GABAergic inhibitory neurons and a few cholinergic neuromodulator cells, almost all receiving cortical inputs (Fig. 1). Ninety-five percent of these GABAergic cells form the more or less anatomically homogenous group of medium spiny neurons (MSNs), but the remaining five percent of aspiny GABAergic cells were recently described as a mixture of at least eight morphologically, neurochemically and electrophysiologically different classes of cells (Tepper et al., 2010). So far, these latter types were only distinguished in slices and intracellular recordings of anesthetized animals. Determining the exact type of the neurons based purely on extracellularly recorded electrophysiological features of their spike trains is almost impossible. 


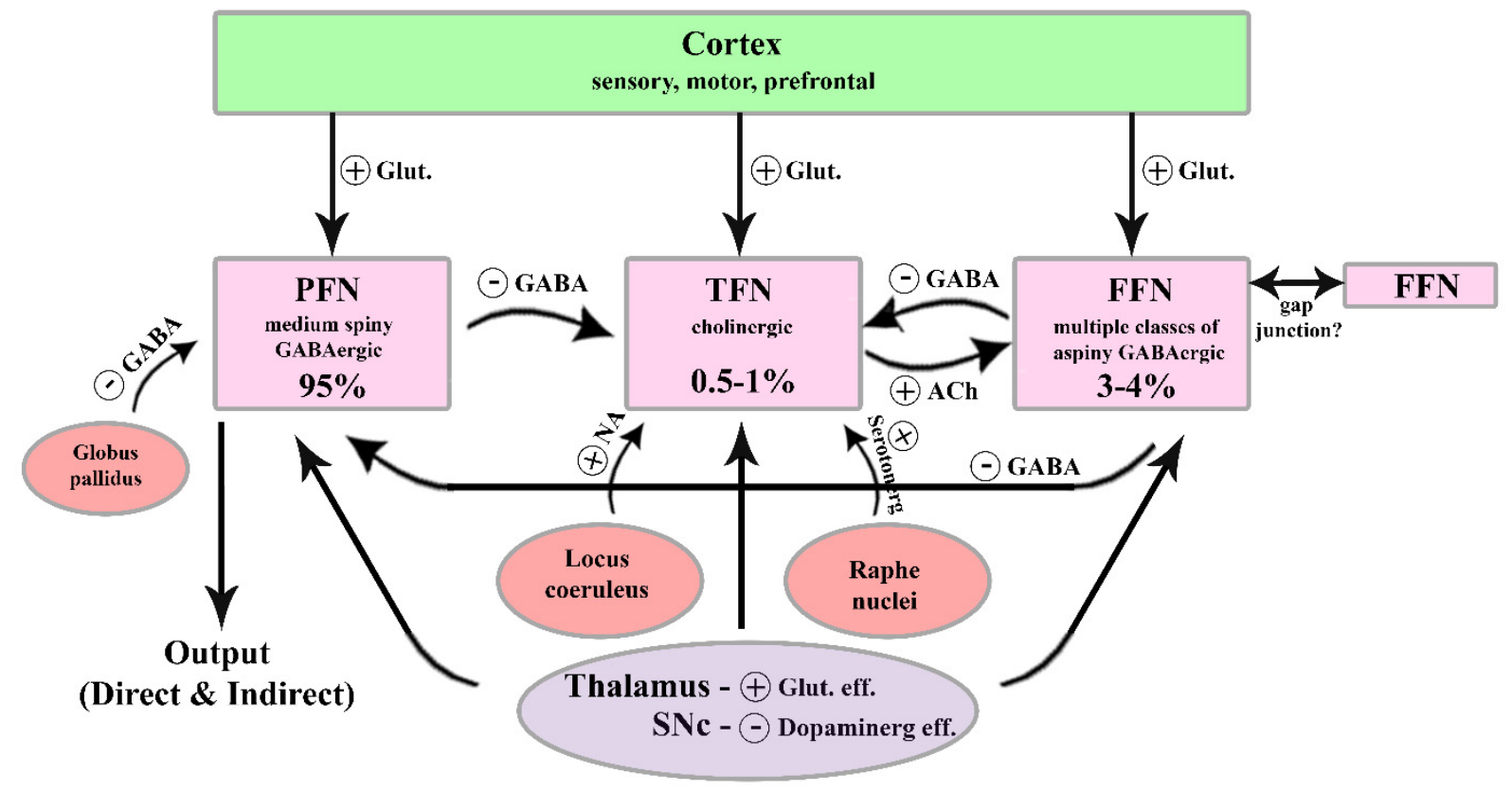

Figure 1. Schematic connectivity diagram of the three main physiological classes of striatal neurons. Abbreviations: SNc, substantia nigra pars compacta; PFN, phasically firing neuron; TFN, tonically firing neuron; FFN, fast-firing neuron

MSNs are divided into two main subpopulations according to their projection via the direct (expressing D1 receptors) or indirect (expressing D2 receptors) pathway (Gerfen et al., 1990). Direct pathway neurons facilitate motor activity, while indirect pathway neurons inhibit it (Reig and Silberberg, 2014). Both subpopulations receive glutamatergic inputs from the thalamus and dopaminergic inputs from the substantia nigra pars compacta (SNc). The anatomical group of MSNs are traditionally identified electrophysiologically as phasicallyfiring neurons (PFNs); however, this group is not homogenous regarding neither it's receptorrepertoire and connectivity (D1 and D2 neurons) (Chuhma et al., 2011), nor its behavioral correlates (Hernandez et al., 2013). Some studies found that the nigrostriatal activation of D1like receptors increases, while D2-like receptors decrease membrane excitability of striatal neurons (Zhou, Wilson and Dani, 2002). 
Cholinergic interneurons make up only $0.5-1 \%$ of the striatal neurons. These neurons are massive integrators; while they receive extensive glutamatergic excitation arising from the cortex and the centromedian and parafascicular thalamic nuclei, they are also modulated by excitatory serotonergic (Bonsi et al., 2007), noradrenergic (mainly in the ventral striatum) (Delfs et al., 1998), and inhibitory dopaminergic inputs (Bonsi et al., 2011). At the same time, they are also deeply embedded in the local intrastriatal circuitry by receiving inhibitory GABAergic innervation from both MSNs and aspiny GABAergic interneurons (Aosaki et al., 2010). As they maintain a relatively stable firing rate and they rarely emit bursts of action potentials, traditionally, they are classified in the electrophysiological recordings as tonically firing neurons (TFNs) (Apicella et al., 1991; Aosaki et al., 1994; Apicella et al., 1998). These cholinergic neurons can control the striatal dopamine release through the modulation of the nigrostriatal axon terminals and can excite both MSN and other GABAergic neurons through nicotinic receptors (Licheri et al., 2018).

The remaining $\sim 5 \%$ of the striatal neurons is composed of various subtypes of aspiny GABAergic interneurons expressing calcium-binding proteins parvalbumin (PV) or calretinin (CR), neuropeptides like somatostatin (SO), neuropeptide Y (NPY) or nitric oxide synthase (NOS) (see (Tepper et al., 2018) for a review). The PV-positive neurons of this group are classified in intracellular recordings as fast-spiking interneurons (FSIs) (Kawaguchi, 1993; Koós and Tepper, 1999), while the other classes are not convincingly classified yet. Striatal FSIs are electronically coupled by gap junctions (Russo et al., 2013). Previous studies found that these gap junctions serve more like brakes by shunting the postsynaptic charges instead of inducing hypersynchrony (Russo et al., 2013). Consequently, the network of FSIs act as filters, because they transduce information only if multiple inputs are present synchronously along the network (Koós and Tepper, 1999) while they can also precisely control spike timing in MSNs via GABAA signaling at perisomatic synapses. In studies based on extracellular electrophysiology measurements, these cells are grouped as fast-firing interneurons (FFNs); however, there is no one-to-one equivalence between FSIs and FFNs; latter is likely 'contaminated' by other aspiny GABAergic classes with more-or-less steady activity patterns (Berke, 2011). 


\subsection{Technical challenges of the analysis of behavioral correlates of striatal neuronal networks.}

Identifying these rare, sparsely occurring cell types and observing their contribution to network activity patterns in behaving animals require the simultaneous observation of a large number (i.e., hundreds) of neurons. While mapping the connectome of brain networks is advancing quickly (Lichtman and Denk, 2011) and innovations of perturbation methods (e.g., optogenetics, DREADDs, etc.) provide toolsets to identify and probe circuit components (Boyden et al., 2005; Lein et al., 2007; Yizhar et al., 2011; Madisen et al., 2012; Prakash et al., 2012; Tye and Deisseroth, 2012), recording methods for monitoring the activity of large numbers of neurons in local and global circuits in the behaving animal are lagging (Nicolelis $e t$ al., 1997; Buzsaki, 2004a; Alivisatos et al., 2012).

High-density, nano-machined microelectrodes ('silicon probes') (Wilson and McNaughton, 1993; Logothetis, 2003; Buzsaki, 2004a; Blanche et al., 2005; Du et al., 2011; Buzsaki, Anastassiou and Koch, 2012) offer the possibility to record electrical activity of local circuits from multiple structures at high temporal resolution and at the spatial resolution of single neurons in freely behaving animal. Moreover, these extracellular electrical recordings also give access to subcortical structures, not easily accessible with calcium imaging. While silicon probe technology is offering ever-larger site numbers (Du et al., 2009, 2011), its use in freely moving animal experiments is constrained by the burdens of the recording electronics. Larger channel count also scales the number of preamplifiers and connecting wires needed and quickly reaching a bulkiness that limits the movements of the animals. Consequently, most network level, large channel count recordings of the brain have been performed on either anesthetized or head-fixed preparations.

Sensory-motor processes, for example, visual, vestibular, proprioceptive, and whisker feedbacks of the self-motion can significantly alter network dynamics. Thus, in systems neuroscience experiments, the unrestrained behavior of the animals shall preferably be preserved. This requirement called for improvements and miniaturization at the level of headstage interconnects, signal multiplexing, the ultra-flexible connection between the animal and the recording equipment (Du et al., 2011; Szuts et al., 2011; Vandecasteele et al., 2012). Signal multiplexers can effectively 'compress' neuronal signals, and transmit them using tethers with 
less constraint, but previously used multiplexers had either low channel counts or they could not simultaneously record both unit and LFP signals due to their high- or low-pass filtering constraints (Olsson 3rd et al., 2005; Harrison, 2008; Du et al., 2011; Szuts et al., 2011; Viventi et al., 2011). During my doctoral work, we developed a high throughput integrated microelectronic system, including high-density, multiple-shank recordings of unit activity and LFP from multiple brain regions, using on-stage signal multiplexing methods. We demonstrated its features by investigating the multimodal integrative capacities of the striatal neuronal networks in freely moving rats.

\subsection{The integrative role of the CPu}

Although the principles of local connectivity of the striatal neuronal classes are preserved throughout the whole striatum, and their cortical inputs show massive convergence, detailed functional studies revealed that still there is a well-expressed functional segregation within the $\mathrm{CPu}$. While the ventral part is mainly associated with reward and motivation related processes (e.g., reinforcement learning, Kravitz et al., 2012), the dorsal striatum is involved in executive functions based on stimulus-response learning (Horvitz, 2009). The latter gives a framework to spatial working memory, also called procedural memory (Pennartz et al., 2011; Akhlaghpour et al., 2016).

The cooperation of the mediodorsal $\mathrm{CPu}$ and the hippocampus promotes action selection based on learned cognitive-spatial information, especially in cases where competitive cues are present (Devan and White, 1999). This observation is confirmed by a recent study of patients with focal ischemic lesions in the dorsal striatum, who performed worse in tasks involving speed-accuracy tradeoffs, suggesting that they were less capable of incorporating sensory percepts in the decision making processes (Winkel et al., 2016). Similarly, an fMRI study of patients with Huntington's disease revealed decreased striatal activity selectively for only some aspects of visual scenes (Nasr and Rosas, 2016). The $\mathrm{CPu}$ is likely to be operating on a rather complex, preprocessed representation of a multisensory environment. Still, to test the effect of these composite inputs on the striatal circuitry, the most promising way remained to investigate one-by-one how the individual sensory features and modalities are transferred to and processed by the striatal network. 
Motion or salient elements and stationary features of a visual scene are dissociated at very early stages of the visual system and processed further as separate streams (Goodale and Milner, 1992), however it seems that these two distinct representations (or at least some aspects of them) are simultaneously present in the $\mathrm{CPu}$. Flashing light could induce evoked responses in the striatal local field potential (LFP) in mice (Reig and Silberberg, 2014), and other studies revealed that a population of neurons in the primate caudate are sensitive to the 'what' and 'where' components of stationary images to select and guide high valence saccadic events (Yamamoto et al., 2012). Motion sensitivity of CPu neurons was also reported in cats (Nagypál et al., 2015) and monkeys (Yanike and Ferrera, 2014).

Distinguishing whether the visual dislocation of a perceived image happens due to the observer's self-motion or due to a moving object indeed, is a complex but crucial task to guide the behavior properly (Cuturi and Macneilage, 2014). Evolution offers a rather conserved solution to this issue: Monitoring a corollary discharge - an internal copy - of the motor commands helps to filter out the sensory consequences of a self-action (Sperry, 1950; Crapse and Sommer, 2008). Saccade induced sensory inputs are regulated in mammals by a tectothalamocortical (frontal eye field) corollary discharge pathway (Sommer and Wurtz, 2002); however, it is not known yet if a similar mechanism is in place for the complex recognition of moving objects and related action selection. From an evolutionary point of view, detecting moving objects in a calm environment when the sensory representation of the latter is not steady either has high importance; this information is guiding movements when reaching for prey or trying to avoid a predator. The striatum is a candidate to support such decisions, as it also receives proprioceptive and vestibular feedback, in addition to visual inputs (Hitier, Besnard and Smith, 2014).

There is no consensus in the literature regarding the origin of visual information that reaches the $\mathrm{CPu}$. Some studies suggest that the visual information is conveyed from the primary or secondary visual cortical areas (Khibnik, Tritsch and Sabatini, 2014; Reig and Silberberg, 2014), while some others suggest that the information is reaching through the visual associative cortex (Nagy et al., 2011) or through subcortical shortcuts, avoiding the visual cortex (for review, see Rokszin et al. (2010)). A common bottleneck of these reports is that they were all performed either in anesthetized or restrained animals, thus only a subdomain of the visual information processing was examined, and they were not suitably designed to investigate the self-motion induced components of the visual motion perception. 


\section{THE AIMS OF MY DOCTORAL WORK}

The central goals of my doctoral work were to examine the response characteristics of the dorsal $\mathrm{CPu}$ neurons in different visual environments and to observe to what extent does selfmotion influences the visual experience. For this, we initially developed and validated the technical requirements to simultaneously perform a controlled visual motion stimulation and large-scale high-density extracellular recordings from multiple brain areas in freely moving rats. In my current doctoral thesis, I summarized my experimental work and the results I reached to decipher 1) which aspects of visual motion information are reaching the caudate nucleus, 2) what is the origin of these inputs, and 3) how these inputs shape the activity of striatal neurons.

My specific aims were the followings:

- To identify the anatomical localization of visual motion-sensitive neurons in the $\mathrm{CPu}$ by detecting immediate-early gene products.

- To validate our newly developed multiplexed high-density extracellular recording technique in order to perform a large-scale unit activity and LFP recordings in animals moving freely during visual stimulation.

- To develop experimental approaches to control visual motion percepts in unrestrained animals.

- To quantify the sensitivity of the dorsal $\mathrm{CPu}$ neurons to self-independent and self-induced visual motion in unrestrained, freely moving rats.

- To identify the possible sources of the striatal visual information by tract-tracing, immunohistochemical methods, and electrical stimulation.

- To confirm the visual motion-related functional connectivity between the striatum and its identified input structure, and to describe the role of this pathway in shaping the visual-motion related visual responses.

As the purpose of this work is to give a compiled overview of my doctoral work, for better readability, I decided to briefly explain the methodology inline as presenting the main findings of my experiments, and I'll describe the precise technical details as the last chapter. 


\section{RESULTS}

\subsection{Dorsomedial striatum receives innervation from the visual cortex}

We performed our experiments on male Long-Evans rats $(\mathrm{N}=32,285-655 \mathrm{~g} ; 3-12$ months old). The multisensory integration capacity of neurons in the dorsomedial striatum has already been described in other species (Strecker et al., 1985; Graziano and Gross, 1993; Reig and Silberberg, 2014), and the intermediate part of the striatum along its rostrocaudal axis was reported to receive axons from both A18 and A18b secondary visual cortical areas, and to be the exclusive recipient of innervation from the primary visual cortex (V1) (Serizawa et al., 1994; Khibnik, Tritsch and Sabatini, 2014). To confirm this finding, we injected Fluoro-Gold to the dorsomedial $\mathrm{CPu}(\mathrm{N}=4$ rats) to identify regions with retrogradely labeled neuronal somata (Fig. 2A). Similarly to previous results obtained in mice and rats (Serizawa et al., 1994; Reig and Silberberg, 2014), we found that the neurons of the most rostral parts of the primary and secondary visual cortices (V1 and V2) sent strong projections to the injected area of $\mathrm{CPu}$ (Fig. 2A). Based on their large somata, their thick apical dendrites, and their restricted location to layer $\mathrm{V}$, the labeled neurons are putative pyramidal cells. We did not find labeled neurons at the caudal parts of the V1 nor the lateral geniculate nucleus (LGN), however, the lateralposteromedial and posterior nuclei of the thalamus were densely labeled.

The next step was to colocalize axons that originate from the visual cortex; we set out to strengthen the evidence of a functional cortical visual input to the visually responsive $\mathrm{CPu}$ neurons. We injected BDA at the same locations as the V2 described above. After one week of survival, the animals were placed into a $60-$ by- $60 \mathrm{~cm}$ large box whose $35 \mathrm{~cm}$ tall walls were constructed of four LCD monitors. Each stimulation trial began with presenting a non-patterned grey background for $500 \mathrm{~ms}$ (Blank screen), followed by $500 \mathrm{~ms}$ of sinusoidally modulated stationer grating pattern (Stationary grating). Lastly, the pattern was gradually moved perpendicularly to the grating's axis (Moving grating) for another $500 \mathrm{~ms}$. The spatial density of the grating and the velocity and direction of the motion was varied in a pseudorandom order, until each parameter combination was repeated at least 150 times (Clifford and Ibbotson, 2002; Gombköto et al., 2011; Nagy et al., 2010). After either seven hours of sensory deprivation ('control animals') or six hours deprivation followed by one-hour visual stimulation ('stimulated animals'), the rats were immediately over-anesthetized and perfused (Fig. 2B). Coronal sections of the brains were developed to visualize BDA positive axons and the expression of the cFos immediate-early gene protein in the neurons (Fig. 2C). 
A

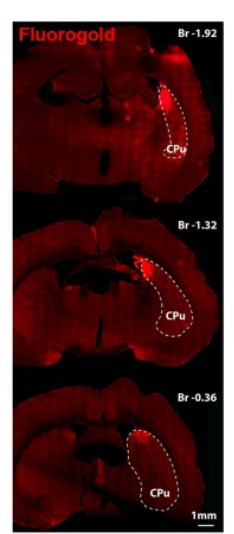

D

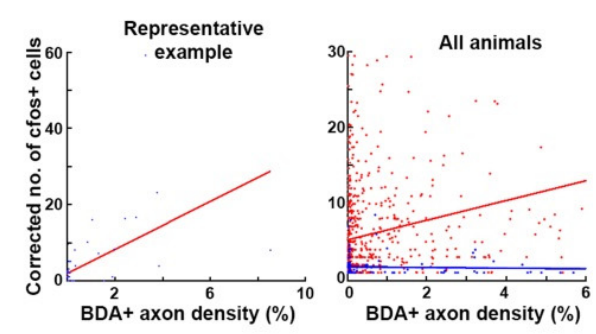

B

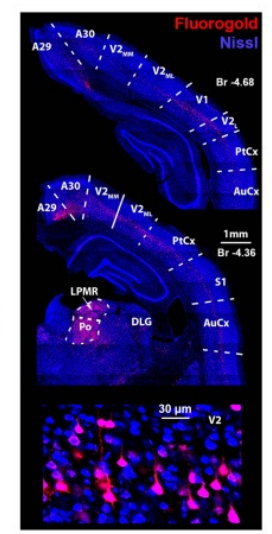

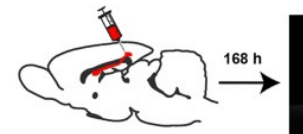

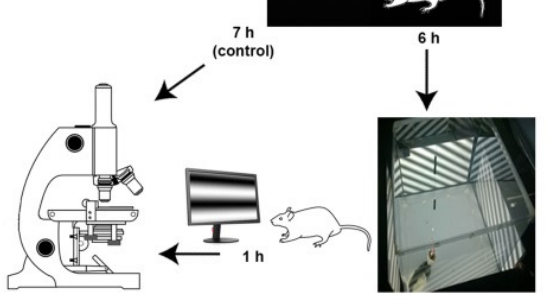

C
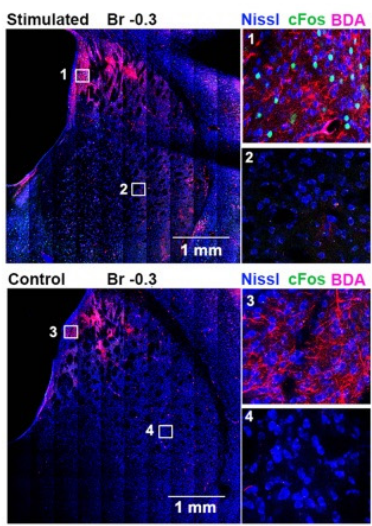

$\mathbf{E}$

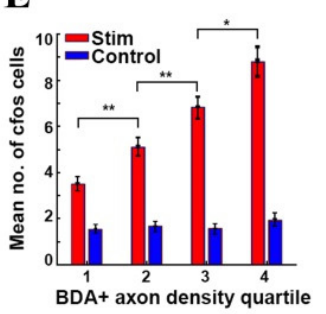

$\mathbf{F}$

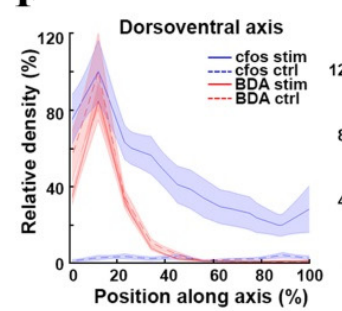

Mediolateral axis

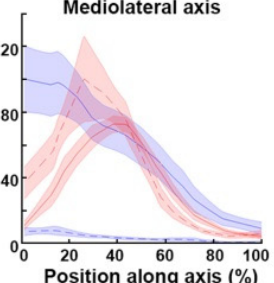

Figure 2. (A) Representative micrographic images of the injection sites of the retrograde tracer Fluoro-Gold in the dorsomedial striatum. Retrogradely labeled cell bodies were found in the primary and secondary visual cortices ipsilaterally, as well as in the posterior (Po) and lateral-posteromedial (LPMR) thalamus. Note the lack of labeling at the dorsolateral geniculate nucleus (DLG). (B) Experimental schematics. (C) Representative example sections of a visually stimulated (upper panels), and a control animal (lower panels). Blue, red, and green color channels are assigned to the fluorochromes of the fluoro-Nissl, BDA, and cFos immunohistochemistries, respectively. (D) Correlation of the normalized cFos and BDA densities in a representative stimulated animal (left panel) and in all animals (right panel). The red and blue colors represent data from the stimulated and sham animals, respectively. (E) Mean number of the $\mathrm{cFos}^{+}$neurons in the striatum calculated for the 4 BDA axon density quartiles. (F) cFos (blue) and BDA (red) density distribution in stimulated (solid lines) and control animals (striped lines). Left and right panels show the distributions along the respective DV and ML axes of the striatum.

Automated, unsupervised algorithms were used to quantify the BDA+ axon density and the number of cFos + neurons in 425 -by- $425 \mu \mathrm{m}$ squares. We found that axons originating from V2 neurons colocalized with cFos + neurons; BDA+ axon density was spatially correlating with the cFos + density in the stimulated animals (Stimulated: $\mathrm{R}=0.20, \mathrm{p}<0.005, \mathrm{~N}=696$ density pairs from five animals; Control: $\mathrm{R}=-0.03, \mathrm{p}=0.69, \mathrm{~N}=134$ density pairs from four animals, 
Pearson's linear correlation, Fig. 2D). The cFos expression was a result of the visual stimulation, as it was uniformly low at every location in the control animals (Stimulated: $3.51 \pm 0.30,5.12 \pm 0.42,6.84 \pm 0.50,8.82 \pm 0.66 \mathrm{cFos}^{+}$neurons found at consecutive BDA density quartiles, $\mathrm{p}=0.002,0.009,0.019$ for interquartile comparison, $\mathrm{N}=4 \times 174$ density values; Control: $1.55 \pm 0.19,1.69 \pm 0.19,1.59 \pm 0.22,1.94 \pm 0.28 \mathrm{cFos}+$ neurons found at consecutive BDA density quartiles, $\mathrm{p}=0.61,0.72,0.33$ for interquartile comparison, $\mathrm{N}=4 \mathrm{x}$ 34 density values; two-sample t-test; Fig. 2E). Both the BDA+ and cFos + locations were restricted to the dorsal part of $\mathrm{CPu}$, however, regarding the mediolateral extent, cFos activity was present at the medial and intermediate locations, while BDA was present mainly in the intermediate locations (Fig. 2F). Almost none of the cFos + neurons were parvalbumin-positive (anti-cFos and anti-PV double immunostaining in four sections of one rat, $\mathrm{N}=490$ and 406 positives for anti-cFos and anti-PV respectively, and only three double-positive neurons, data not shown).

In our preliminary experiments, we initially attempted to perform these studies on anesthetized rats, in combination with the recording of evoked single-unit responses during the visual stimulation. Surprisingly, despite the unequivocal innervation from the visual cortex, we could induce neither cFos expression nor any evoked neuronal response. The effect of visual stimulation was barely visible even in the stimulus triggered by evoked LFP waveforms. These findings stress the importance of recording visually induced neuronal responses in awake, freely moving animals. To analyze the intrastriatal connectivity as well as the visual information transfer from the cortex to the striatum requires acquiring a statistically meaningful number of neurons from multiple brain regions at the same time. To achieve this, we decided to perform extracellular recordings using multishank silicon electrodes, which can capture the activity of dozens of neurons simultaneously from deep brain regions also and can be iteratively advanced to sequential recording locations without significant tissue damage (Vandecasteele et al., 2012). A major challenge of the effective use of silicon probes in small animals is to reduce the volume and weight of the instrumentation (such as preamplifiers, cabling, etc.) between the probe and the recording equipment. 


\subsection{Large-scale extracellular recordings using multiplexed signal acquisition}

By the time of our experiments, recording signals from more than 32 or 64 recording sites were seriously constraining the behavior of the rats, due to the bulkiness of the needed channel-by-channel preamplifiers, and the corresponding thick cablings. To overcome this obstacle, we decided to develop an electrophysiology recording apparatus by deploying timedivision multiplexing to achieve miniaturization and signal compression (Olsson 3rd et al., 2005; Harrison, 2008; Szuts et al., 2011; Viventi et al., 2011). We decided to implement this concept using a 32-channel VLSI analog signal multiplexer ICs recently explicitly developed for biosignal acquisition purposes (Harrison, 2008) (RHA2132, INTAN Inc., www.intantech.com). To allow movement of the probe and preserve its small inertia, an ultraflexible polyimide-based cable was designed and served as an interconnect between the silicon probes and the multiplexing headstage. For the technical details of the implemented data acquisition solution, see Materials and Methods.

To validate this recording system, we compared the signal quality to a commercially available recording system (HST/32V-G20) preamplifier (Plexon Inc, Dallas, TX, USA) and Digital Lynx 16SX A/D converter (NeuraLynx Inc, Bozeman, MT, USA), serving as industrial standards. When the input channels were short-circuited with the reference pin of the headstage, the mean root-mean-square amplitude of $60 \mathrm{~Hz}$ noise was $7.2 \mu \mathrm{V}$ (range $=2-8.5 \mu \mathrm{V}$ ), which is approximately one order of magnitude higher than the half least significant bit of the A/D conversion, and was comparable to the reference system's noise level. The common noise component across channels in the recorded LFPs was significantly lower, especially in the lower frequency ranges, which is presumably a result of the serial transmission of the channels in the multiplexed line instead of the nonmultiplexed parallel transmission. The full range amplitude of the signal was approximately $20 \%$ smaller using the INTAN amplifier chips than that of the non-multiplexing control system, which can be explained by the lower input impedance of these chips. The mean correlation across the shanks gradually decreased at higher frequencies in our system $(\mathrm{R}=0.8,0.72$ and 0.23 for $>1,>10$, and $>100 \mathrm{~Hz}$, respectively), while slightly increased for the control system $(\mathrm{R}=0.55,0.72,0.75)$. We also recorded the same signals using both systems simultaneously by splitting the electrode leads before amplification. The single unit yield was larger in our system, more than triple single units were detected on a single shank, using our (test) and the control systems. Using this implementation 
of multiplexed signal recording, we could successfully demonstrate the feasibility of long-term 256 and 512-channel recordings in freely moving rats, and 64-channel recordings in mice (Figures 3 and 4).
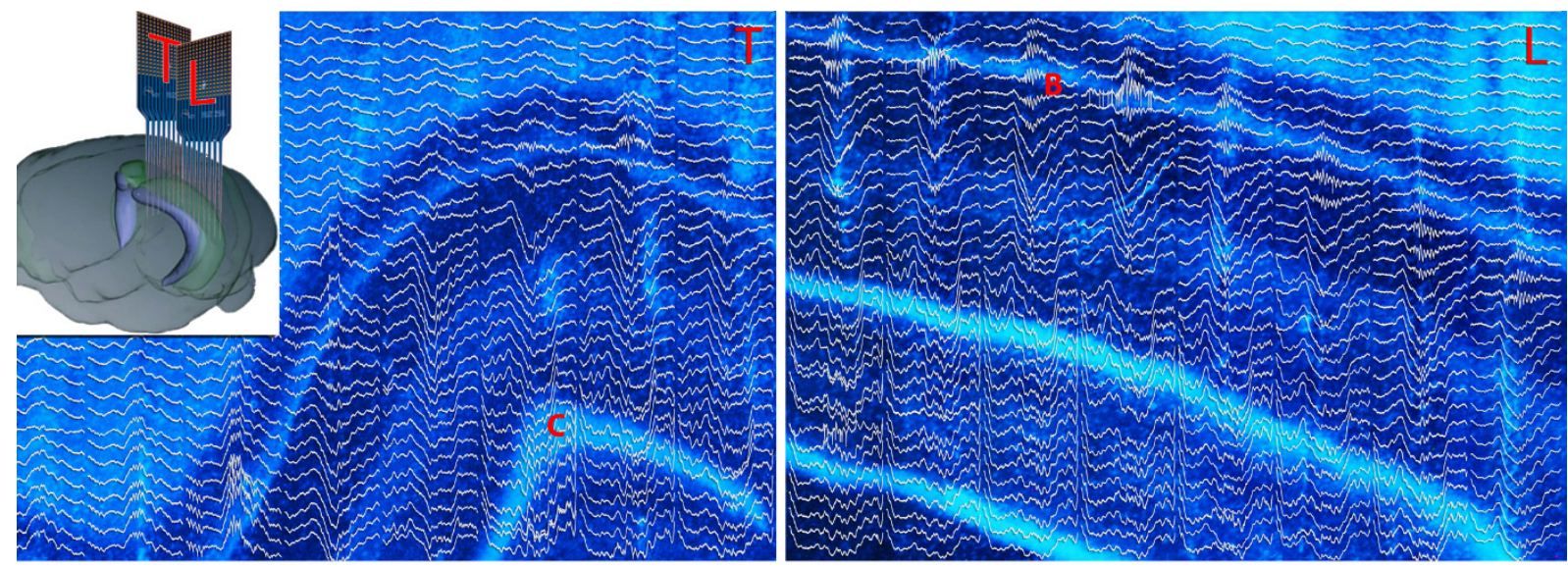

Figure 3. Chronic, 512-channel recordings in a freely moving rat. The figure shows the histological reconstruction of the hippocampal crosssections at the recording planes with superimposed traces of an SWR event (300 ms). Inset: orientation of 2 probes placed in the same hippocampus in the transversal $(\mathrm{T})$ and longitudinal $(\mathrm{L})$ axes. ' $\mathrm{B}$ ' and ' $\mathrm{C}$ ' denotes two example landmark positions of cellular layers where maximal spike amplitudes were detected.

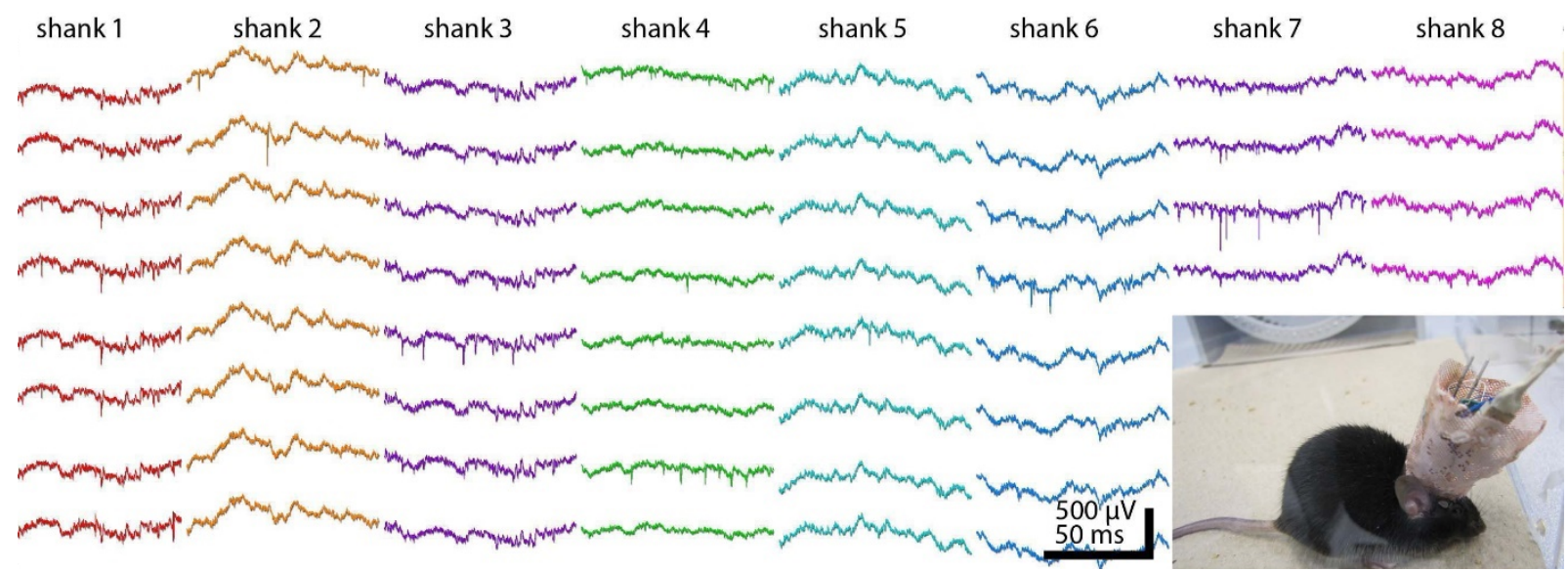

Figure 4. Chronic unit and LFP recordings from a freely moving mouse using an 8-shank 64-site silicon probe. One hundred-millisecond epoch from each shank are shown. Inset: headstage with silicon probe, microdrive, and 64-channel signal multiplexer surrounded by copper mesh shielding. 


\subsection{Identity and connectivity of the recorded cells in the dorsomedial striatum}

To test the responsivity of the striatal neurons to moving visual stimuli, we performed large-scale extracellular recordings with the help of the aforementioned multiplexed data acquisition approach, while the animals were exposed to the same visual stimulation protocol as in the cFos expression experiments (Fig. 2B). Using either tetrodes or silicon electrodes ('silicon probes'), we recorded the activity of altogether 734 neurons from unrestrained rats performing various behavioral and visual tasks $(\mathrm{N}=42$ sessions in six animals). The recorded single units were isolated from the compound electrical activity based on their action potential waveforms using a semi-automated clustering method (Kadir, Goodman and Harris, 2014; Rossant et al., 2016). Noisy units or multiunits were discarded, while clusters meeting our predefined isolation quality criteria (Berényi et al., 2014) were classified based on their electrophysiological features as phasically firing (PFNs; $\mathrm{N}=467 ; 63.62 \%$ of all isolated single units), fast firing (FFNs; $\mathrm{N}=163 ; 22.21 \%$ ) and tonically firing neurons (TFNs; $\mathrm{N}=56$; $7.63 \%$ ). These classes putatively match the morphological and neurochemical groups of medium spiny, parvalbumin-positive, and cholinergic aspiny neurons, respectively (Barnes et al., 2005; Berke, 2008). In six percent of the recorded single units, the classification was ambiguous due to their meager firing rate, and those units were discarded from further analysis. The overrepresentation of FFNs in our recordings compared to the reported anatomical proportions (Oorschot, 2010) was attributed to the less reliable classification of the more sparsely firing PFNs in short sessions, as they either were missed during the manual cluster refinement or were classified as 'ambiguous/unknown'.

The recorded neurons were sparsely connected. Our cross-correlogram-based analysis revealed a relatively large number of electrical synapses between fast-firing neurons $(6.77 \%$ of all theoretically possible pairs, Vandecasteele et al., 2007), and a monosynaptic excitation exerted by the TFNs on FFNs (4.84 \% of all possible pairs), but not on PFNs (Luo et al., 2013). FFNs caused the very few detected inhibitory interactions on PFNs or FFNs. 


\subsection{Striatal responses to self-independent visual stimulation}

In the next step, we mapped the response profiles of the recorded striatal neurons to the visual stimulation paradigm (Fig. 2B). Neurons were considered responsive if they significantly altered their firing rate during either the Stationary or the Moving condition for at least two of the parameter combinations. Twenty-five of the 289 neurons were considered responsive to this passive, self-motion independent visual stimulation ( $\mathrm{pVis}$ stimulation, $\mathrm{pVis}+$ neurons). In general, we found no systematic preference toward any of the tested directions of motion, and some neurons responded similarly to all directions, while others preferred only a few adjacent directions or two opposing ones (Fig. 5A).

Regarding the spatial resolution of the grating and the velocity of the motion, neurons were more sensitive to broader stripes sliding with a high velocity compared to the dense, slowly shifting gratings $(\mathrm{p}<0.005$ for all comparisons but thick vs. medium moving stripes, where $\mathrm{p}=0.92 ; \mathrm{N}=200$ in each group; paired t-test). To describe the general responsiveness of these neurons, we averaged the modulation indices of the stationary and moving conditions (calculated against the blank condition) across all stimulus conditions. Surprisingly, the response combinations occupied only a restricted subset of the available state-space: the $\mathrm{pVis}+$ neurons either increased their firing in the presence of the stationary grating or became inhibited by the moving gratings or the combination of these (Fig. 5B).

A

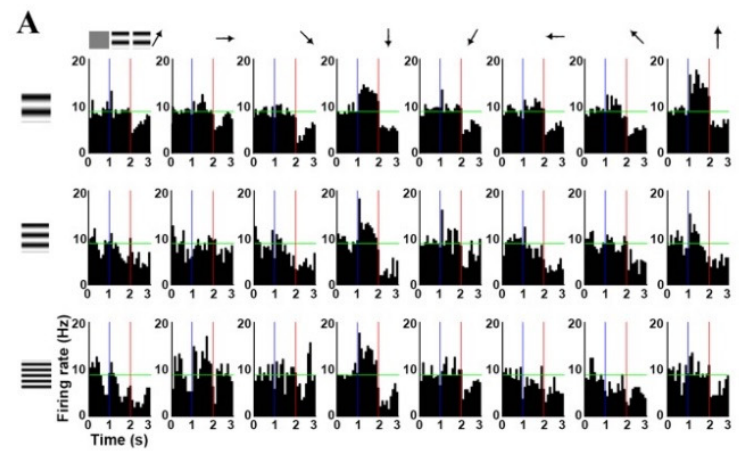

B

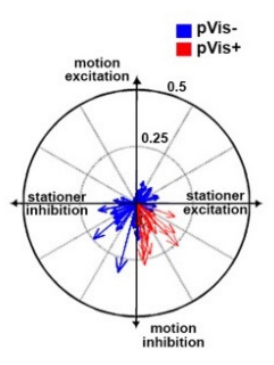

C

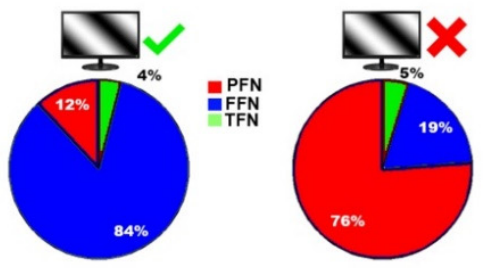

Figure 5. (A) PSTHs of a pVis + striatal neuron's responses during visual stimulation using $3 \times 8$ parameter combinations. The first third of each PSTH represents the activity of the neuron while watching a uniform grey image, middle and right third reflects the responses during still and moving stripes, respectively. Horizontal green lines represent the spontaneous firing rate of the same neuron. (B) Response vectors of $p V i s+$ (red) and pVis(blue) neurons. Modulation indices (MIs) of the 24 stimulus conditions are averaged for each cell, and still and moving MIs are assigned to the $\mathrm{X}$ and $\mathrm{Y}$ components of each response vector. (C) Distribution of cell types among the $\mathrm{pVis}+$ (left) and pVis- (right) neurons. 
To verify the cortical innervation of the recorded neurons, we delivered brief electric impulses to V2 using a bipolar stimulation electrode and evaluated the stimulation-triggered response profiles of the striatal cells (see Materials and Methods). The group of $\mathrm{pVis}+$ neurons and the group of neurons responsive to V2 electrical stimulation (El+ neurons) were strongly overlapping; 20 of $25(80 \%)$ pVis + neurons were sensitive to electrical stimulation, while the same applied to only 14 of $252(6 \%)$ pVis- cells. Importantly, the profiles of the electrically evoked activities showed that both monosynaptic and polysynaptic responses (data not shown).

The majority of both pVis + and El+ neurons were FFNs (84 \%; 12\% PFNs and one neuron was classified as TFN.). In contrast, the distribution of cell types among pVis- neurons resembled the histological proportions of these classes (76 \% PFN, $19 \% \mathrm{FFN}$, and $5 \% \mathrm{TFN}$ ) (Fig. 5C).

\subsection{Striatal responses to self-congruent visual stimulation}

The aforementioned moving visual stimuli were presented while the animals were either standing still or their movements were discordant from the visual input. As these passive visual stimuli may be processed differently from the optic flow of natural scenes, which is concordant with the other senses (e.g., the proprioception), we decided to investigate the visual responsivity of $\mathrm{CPu}$ neurons during self-motion, too. In contrast to experiments on head-fixed animals placed in a virtual environment, we aimed to generate a realistic visual experience congruent with the movements of the animals (active visual stimulation - aVis), which preserves all proprioceptive and exteroceptive aspects of the movements. We trained the animals to run in a linear maze back and forth for water reward. To keep control of the visual environment seen by the rat during each run, we built the walls of the linear maze from translucent plexiglass and placed it in a frame equipped with two perpendicular mirrors along the whole track. An LCD projector projected computer-generated visual scenes onto the walls and the floor of the linear maze with the help of mirrors to avoid shading by the rat. Before each trial, the projected scene was randomly changed to either a uniformly grey ('Blank maze') or a grated pattern ('Striped maze’) (Fig. 10E). 
$685 \mathrm{CPu}$ neurons were tested in this task (41 sessions in five rats, including the neurons, also tested in the $\mathrm{pV}$ is environment), and in marked contrast to the small proportion of $\mathrm{pVis}+$ neurons, 325 of them (62\% PFN, $26 \%$ FFN and $11 \%$ TFN) displayed a significant change in their firing rates in response to the striped environment (aVis+ neurons, Fig. 6A). Importantly, all $\mathrm{pVis}+$ neurons were also responsive in this task; thus the $\mathrm{pVis}+$ cells can be considered a subgroup of the aVis + neurons. For simplicity, from here on, we will call those cells which were only responsive in the $\mathrm{aVis}$ environment but not in the $\mathrm{pVis}$ one (i.e., $\mathrm{pVis}-\mathrm{aV}$ is + neurons) as aVis + neurons. Only 23 of the aVis + neurons modulated their firing rates as a function of the instantaneous running speed $(\mathrm{p}<0.05$, Pearson's linear correlation; number of modulated neurons: 3 of $25 \mathrm{pVis}+, 20$ of $221 \mathrm{aVis}^{+}, 26$ of $\left.233 \mathrm{aVis}-\right)$, and the velocity of the animals were identical in the two environments $(83.3 \pm 18.9 \mathrm{~cm} / \mathrm{s}$ vs. $85.0 \pm 12.3 \mathrm{~cm} / \mathrm{s}, \mathrm{n}=718$ and 649 for blank and striped conditions, respectively; $\mathrm{p}=0.15$, two-sample Kolmogorov-Smirnov test) (Fig. 6B).

A

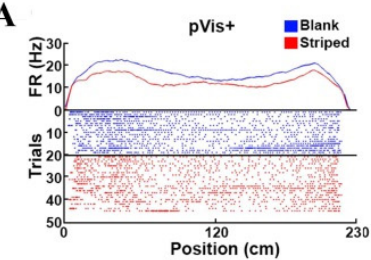

B

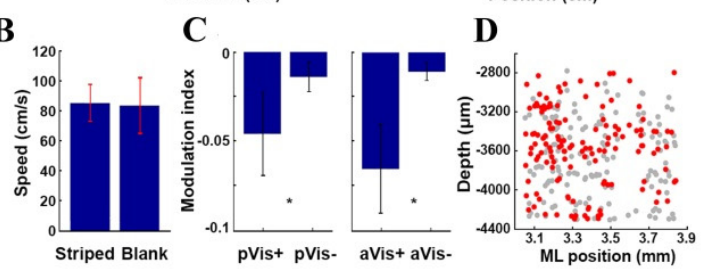

$\mathbf{E}$

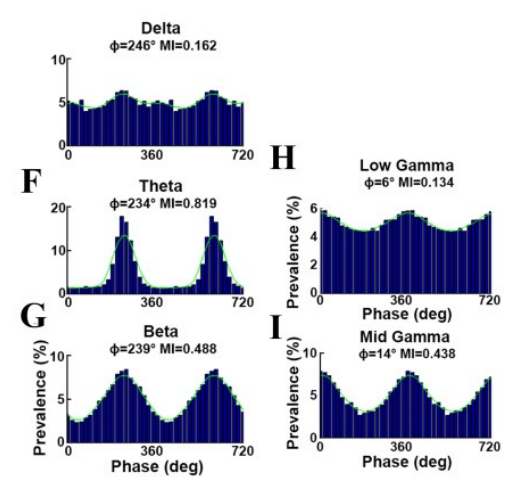

$\mathbf{J}$

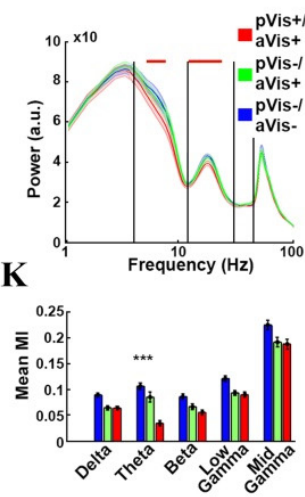

Figure 6. (A) Raster plots and place dependent firing rates of two example FFNs recorded during the same session, simultaneously. Blue dots and curve represent trials during blank maze running; the red ones show the firing pattern during striped maze. (B) Comparison of the mean running speeds in the striped and blank environment. (C) Mean of the modulation indices of pVis + and pVis- neurons (left panel) and aVis + and aVis- neurons (right panel) in the linear maze with the dynamic visual environment. (D) Location of the aVis + neurons (red dots) in the coronal plane. Grey dots denote the location of the non-responsive neurons. aVis + neurons were distributed more uniformly than the pVis + cells. (E-I) Phase coupling of a representative neuron to the delta, theta, beta, low gamma, and high gamma bands, respectively. Mean preferred phase values and modulation indices are shown above each plot. Green curves mark the smoothed distributions. (J) Mean LFP spectral power at locations of the $\mathrm{pVis}^{+}$(red), aVis + (green) and pVis-/aVis- somata (blue). Red horizontal lines show significant segments. (K) Mean modulation indices of the pVis-/aVis- (blue), aVis + (green) and pVis + (red) neurons. pVis + neurons were less coupled to the phase of the theta and beta-band oscillations. 
These two observations led us to conclude that the firing rate change was induced by the altered visual input and not the motion itself. In agreement with their behavior during the passive visual stimulation experiment, $\mathrm{pVis}+$ neurons decreased their firing rate during running in the striped linear maze. The visual modulation of the aVis + neurons was similar to the response profile of the $\mathrm{pVis}+$ neurons $(\mathrm{MI}=-4.7 \pm 2.3 \%$ and $-1.4 \pm 0.8 \%, \mathrm{p}=0.047, \mathrm{~N}=12$ and 31 for $\mathrm{pVis}+$ and $\mathrm{pVis}-$ neurons, respectively, and $\mathrm{MI}=-6.9 \pm 2.7 \%$ and $-1.0 \pm 0.5 \%$, $\mathrm{p}=0.041, \mathrm{~N}=133$ and 50 for aVis + and aVis- neurons, respectively, two-sample KolmogorovSmirnov test) (Fig. 6C), however, their presence was not restricted to the mediodorsal segment of the $\mathrm{CPu}$, but were distributed more uniformly (Fig. 6D).

\subsection{LFP phase preference of the recorded striatal neurons}

Neurons of the CPU were generally phase-locked to the characteristic oscillations of the surrounding local field potentials, suggesting that the recorded units are strongly embedded into the local neuronal networks. An example neuron, displaying particularly strong phase coupling to all investigated frequency bands, is shown in Fig. 6E-I. In order to see if these local ensembles are exposed to and/or generating oscillations of varying intensities depending on their visual responsivity, we compared the spectral profiles of the LFPs at the locations of the $\mathrm{pVis}+$ and $\mathrm{aVis}+$ neurons to those recorded at other locations where we found only $\mathrm{pVis}-/ \mathrm{aVis}-$ neurons. Unexpectedly, we found that low and mid-gamma band power, which are generated mainly by local networks were similar in all three groups $(p>0.05$ for all frequencies within band), but theta and beta powers were significantly weaker at recording locations where $\mathrm{pVis}+$ neurons were present ( $\mathrm{p}<0.05$ for frequencies between $5.5-8 \mathrm{~Hz}$ and $12-25 \mathrm{~Hz}$, paired t-test with Bonferroni correction). Delta band power showed no location-dependent difference ( $p>0.05$ for all frequencies within the band, Fig. 6I).

The $\mathrm{pVis}+$ and $\mathrm{aVis}+$ neurons were in general more weakly coupled to oscillations than the pVis-/aVis- ones (mean MIs, delta: $10.84 \pm 0.50$ vs. $7.89 \pm 0.50$ and $7.85 \pm 0.41$; theta: $12.89 \pm 0.76$ vs. $10.37 \pm 1.16$ and $4.29 \pm 0.65$; beta: $10.48 \pm 0.58$ vs. $8.12 \pm 0.65$ and $6.80 \pm 0.58$; low-gamma: $14.64 \pm 0.64$ vs. $11.36 \pm 0.53$ and $10.93 \pm 0.59$; high-gamma: $26.96 \pm 1.10$ vs. $23.02 \pm 1.12$ and $22.57 \pm 1.12$ for $\mathrm{pVis-/aVis-} \mathrm{vs.} \mathrm{aVis}+$ and $\mathrm{pVis}+$ neurons, respectively), and consistently with the weaker LFP power, this difference in phase coupling was very prominent in case of the $\mathrm{pVis}+$ neurons in the theta band $(\mathrm{p}<0.001, \mathrm{n}=145$ and 25, Kolmogorov-Smirnov test, Fig. $6 \mathrm{~J}, \mathrm{~K})$. Phase preferences of $\mathrm{pVis}-\mathrm{aVis}-, \mathrm{aVis}+$ and $\mathrm{pVis}+$ neurons were similar at all five frequency bands ( $\mathrm{p}>0.05$ for all comparisons). 


\subsection{Phase-locking, space-coding and reward-related activity of the recorded neurons}

The dorsomedial part of the intermediate striatum is known to integrate highly heterogeneous, multimodal inputs in mice (Hunnicutt et al., 2016), thus we were interested if pVis + and/or aVis + neurons possess more integrative features than the rest of the recorded neurons. A significant number of neurons showed spatial location selective activity, similar to the place cells (O'Keefe and Dostrovsky, 1971) of the hippocampal formation. All of these neurons had one single place field similar to the proximal CA1 and CA3c regions of the hippocampus (Oliva et al., 2016); however, their 'place-fields' were relatively large, usually occupying more than one-third of the maze. Although the activity of these 'place-cell-like' neurons was modulated by the presence of striped patterns in the linear maze to a similar extent as the activity of those, which were active at every location, the spatial coding ability of the pVis + neurons were slightly but not significantly worse than the pVis- neurons, and the presence of the striped pattern did not influence it. The spatial coding ability of the aVis + neurons were slightly, but not significantly worse compared to the $p V i s+$ neurons $(p>0.05$ for all comparisons, paired t-test across conditions and Kolmogorov-Smirnov test across neurons; $\mathrm{N}=35$ aVis,+ 31 aVis-, 21 pVis + and 45 pVis- FFNs (Oliva et al., 2016)). The location of the place fields were stable and independent of the presence of the striped pattern, suggesting that the animal considered the two conditions spatially identical, despite the very different visual percept (mean Rho $=0.86 \pm 0.13 ; p<0.05$ in 51 of 53 neurons, Pearson's-linear correlation of 20 spatially binned firing rate pairs per neuron).

The strength of the modulatory effect of stripedness on $\mathrm{pVis}+$ neurons was similar in trials running in one direction in the maze, as while running backwards $(R=0.63, p<0.005$, $\mathrm{N}=22$ modulation index pairs, Pearson's linear correlation). The same applies for pVisneurons too, however, there was slightly more variance between the modulation indices during forward and backward running trials $(\mathrm{R}=0.43, \mathrm{p}<0.05, \mathrm{~N}=57$, Pearson's linear correlation). Similar to hippocampal place cells, a subset of the recorded neurons also possessed phase precession, but due to the very wide place fields, usually the spiking of these neurons precessed more than one full theta cycles, and the space-phase coupling was noisier than hippocampal phase precessing pyramidal cells in general. Phase precession with respect to other frequency bands was negligible. These findings are similar to the properties of ventral striatal neurons (van der Meer and Redish, 2011). 
Although reward-related activity and decision-making are traditionally associated with the ventral striatum, more than half of the recorded neurons expressed some reward-related change in their activity. We detected the moment when the rats reached the water ports with a millisecond precision, but even within a single session, the activity patterns of the neurons were not consistent in terms of their delay and duration (Hernandez et al., 2013). Hence, we could only classify them as inhibitory or excitatory responses. Peri-reward LFP spectra were qualitatively similar at $\mathrm{pVis}+$, aVis + and $\mathrm{pVis}-/ \mathrm{aVis}-$ locations. Theta and mid gamma-band power permanently decreased, while beta, low-gamma, and high-gamma bands $(65-90 \mathrm{~Hz})$ were transiently increased when the animal reached the reward site, especially at the aVis + and pVis + locations. These findings are similar to the peri-reward LFP spectral profile of the ventral striatum (van der Meer et al., 2010), and match with the earlier reports about findings in the dorsomedial striatum (DeCoteau et al., 2007).

Overall, the pVis + neurons were more likely to express place-field-like (36 vs. $18 \%$ ), phase precessing (20 vs. $4 \%$ ), or reward-related activity (91\% vs. $51 \%$ ) than their pVis- peers (Fig. 7A-C). The aVis + neurons were less sensitive to these non-visual modalities $(22 \%, 6 \%$, and $61 \%$ for place-field, phase precession, and reward modalities, respectively). It is worthwhile to note that the majority of the $\mathrm{pVis}+$ neurons responded with a salient inhibition when reaching reward ( $87 \%$ inhibition vs. $4 \%$ excitation), while a solid proportion of the aVis + neurons was also capable of reacting with an excitation $(21 \%$ excitation vs. $40 \%$ inhibition and $40 \%$ non-responsive). The reward sensitive pVis- neurons were either inhibited or excited by the reward in a comparable proportion (29\% vs. $22 \%)$. The number of nonsensitive (35\%, 101 neurons), unimodal (32\%, 92 neurons) and multimodal (33\%, 96 neurons) neurons to the visual, spatial or reward-related sensitivity were similar; however, the $\mathrm{pVis}+$ neurons were more likely found to be multimodal than the aVis + cells (Fig. 7D). 
A

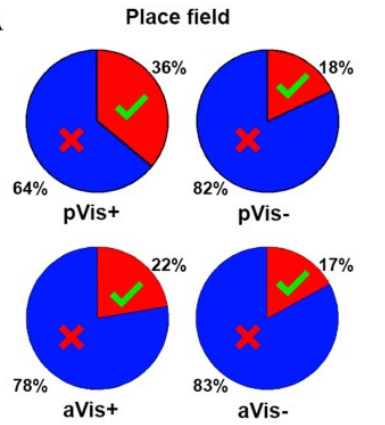

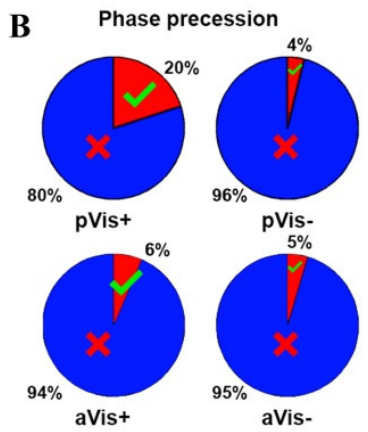

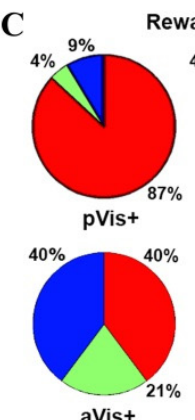

aVis+
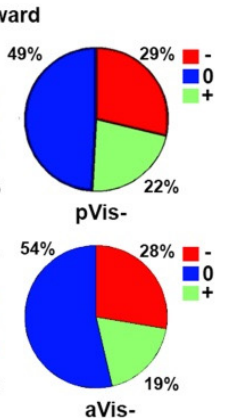
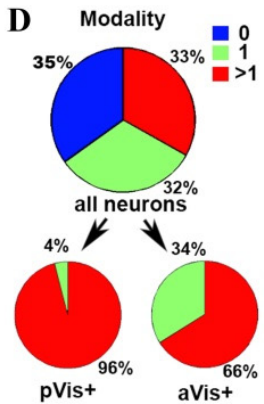

Figure 7. (A) Proportion of neurons with (red) and without (blue) place field like firing pattern among pVis + (top left), pVis- (top right), aVis+ (bottom left) and aVis- (bottom right) neurons. (B) The proportion of precessing (red) and non-precessing (blue) neurons for at least one of the investigated LFP bands. (C) Distribution of the various peri-reward activity patterns among neurons. Blue, green, and red colors denote no change, peri-reward excitation, and peri-reward inhibition, respectively. Panels on ' $\mathrm{B}$ ' and ' $\mathrm{C}$ ' represent the same cell classes as on 'A'. (D) The proportion of non-modulated (blue), unimodal (green), and multimodal (red) neurons in the dorsomedial striatum (top). Lower panels represent the occurrence of uni- and multimodality among pVis + (bottom left) and aVis + (bottom right) cells.

\subsection{Information transfer between the visual cortex and striatum}

We had to restrict our analysis regarding the direct functional cooperation of these structures to local field potential investigations between $\mathrm{V} 2$ and $\mathrm{CPu}$ due to several technical constraints. Although confirming the direct monosynaptic cell-to-cell interactions would provide the ultimate evidence on the information flow between the visual cortex and the $\mathrm{CPu}$, but it is almost impossible to reveal these in intact animals. Cross-correlation based analyses are very unreliable with sparsely firing neurons due to the low number of spikes even if extensive recordings are done for many hours, and optogenetics-based transsynaptic excitation/inhibition is also problematic due to the unknown synaptic transmission efficacy. Moreover, V1 is known to project only to a very thin medial strip of the $\mathrm{CPu}$ along its ventricular wall (Khibnik, Tritsch and Sabatini, 2014), and this is extremely difficult to investigate using our electrophysiological approaches in freely moving animals. 
In two animals, we implanted an additional 32-channel silicon probe into area V2 and recorded both single units and local field potentials while the animals were exposed to the aVis stimulation protocol in the linear maze (Fig. 8A). The frequency spectra of the LFPs recorded at the $\mathrm{CPu}$ and at $\mathrm{V} 2$, and their coherence were identical in striped and blank trials (except 80-90 Hz in V2, Fig. 8B, coherence data not shown), suggesting that the visual environment does not alter gross information transfer between these structures. However, traditional correlation and coherence analyses cannot reveal sparse, package-like increases in the synchrony of discrete frequency bands, especially if they are lagging because of the low correlation level of the asynchronous majority of the signal hides it.

To solve this bottleneck, we developed a time and frequency-resolved cross-coherence estimation, which is particularly sensitive to lagging, intermittent changes in synchrony such as bursty transmissions (Fig. 8C). Using this method, we found that there are transient oscillations in the mid-gamma band that appear coherently in both V2 and the $\mathrm{CPu}$ with a constant time lag (V2 leading with approx. $30 \mathrm{ms,} \mathrm{Fig.} \mathrm{8D).} \mathrm{A} \mathrm{similar} \mathrm{but} \mathrm{weaker} \mathrm{coherent} \mathrm{delayed} \mathrm{pattern} \mathrm{was}$ present between $\mathrm{V} 2$ and the motor cortex (mCtx, Fig. 8E). In contrast to the vCtx-mCtx coherence, which was similar both during running in the blank and the striped maze (Correlation index $=0.058 \pm 0.004$ and $0.054 \pm 0.006$ for blank and striped conditions, respectively; $\mathrm{p}=0.37 ; \mathrm{N}=64$ recording site pairs), the vCtx coherence with the $\mathrm{CPu}$ became significantly weaker when the striped pattern was present (Correlation index $=0.11 \pm 0.004$ and $0.07 \pm 0.003$ for blank and striped conditions, respectively; $\mathrm{p}<0.001 ; \mathrm{N}=96$ location pairs, Fig. $8 \mathrm{~F}$ ), suggesting the presence of a population level communication 'channel' which is influenced by the visual environment. 
A
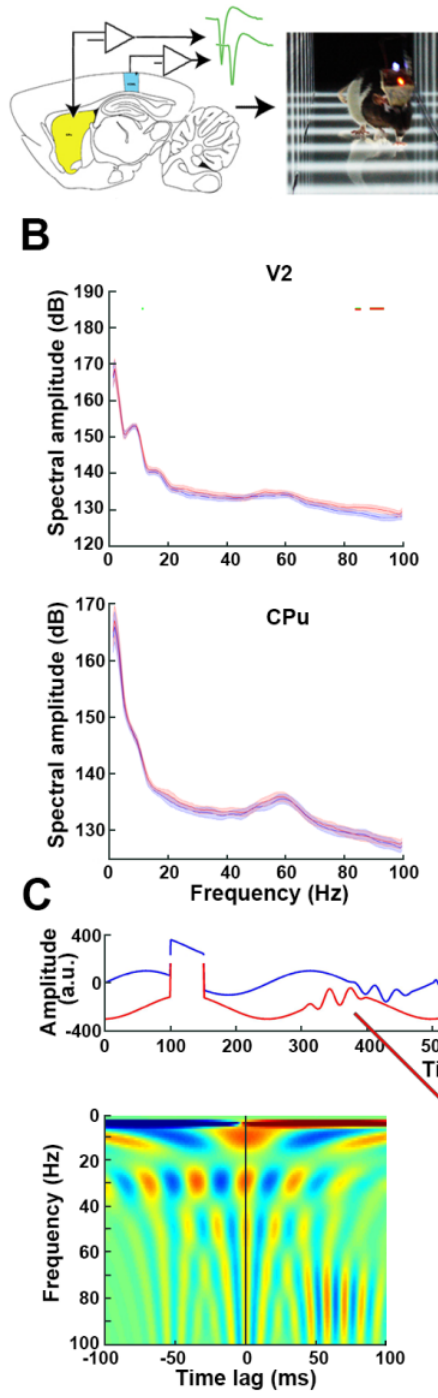
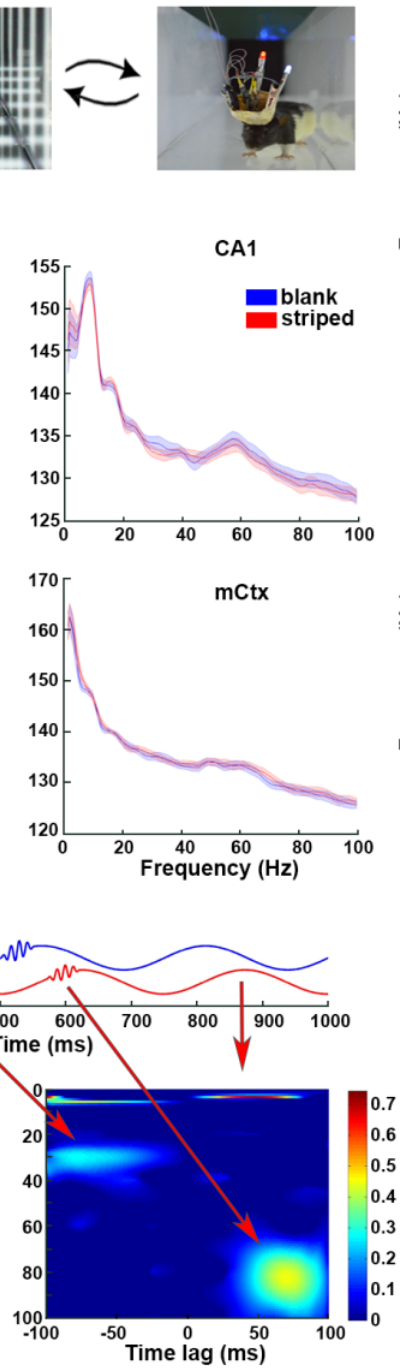

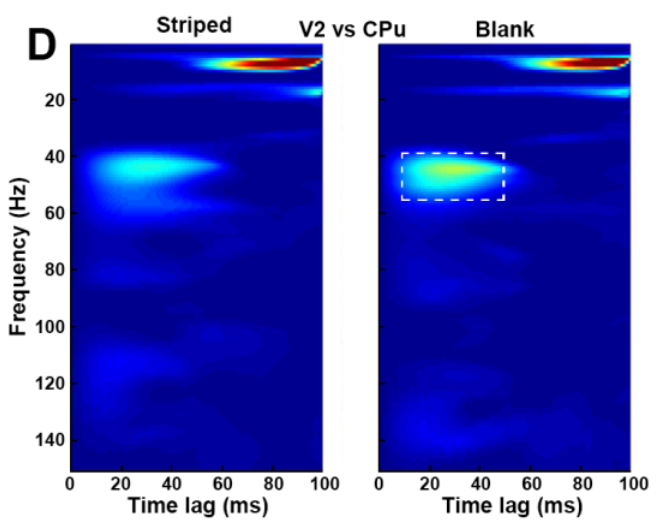

E

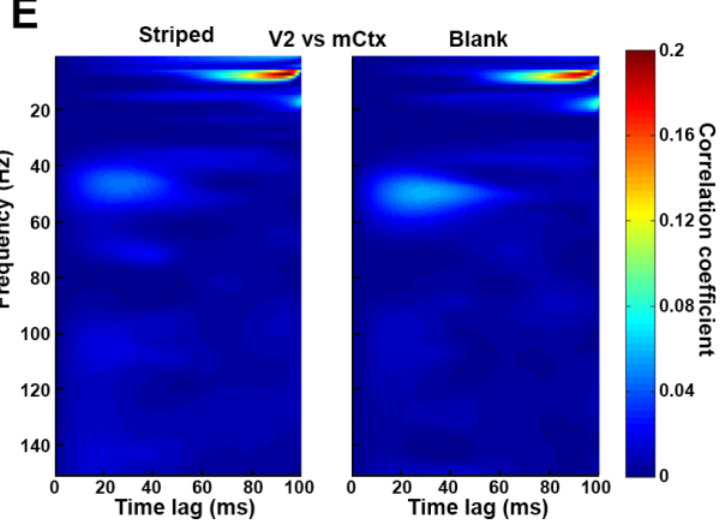

F
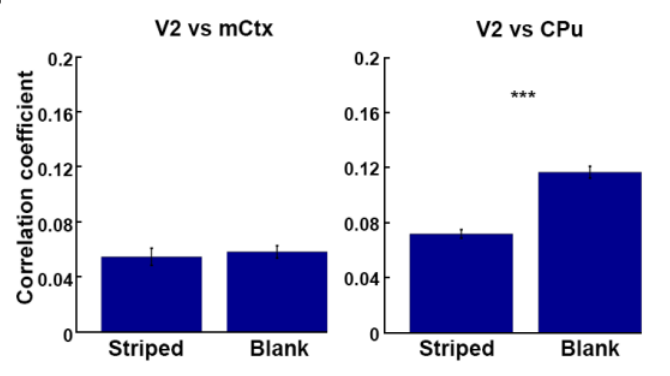

Figure 8. (A) Experimental schematics showing the simultaneous recording of the LFPs and single-unit activity in the V2 and the dorsomedial CPu. (B) Mean raw spectra of the V2 (top left), CPu (bottom left), dorsal CA1 (top right), and motor cortex (bottom right). (C) Demonstration of the concept of our time-lag and frequency-resolved cross-correlation estimation method. (D) The average time and frequency-resolved coherence maps across all sessions and animals for trials in the striped (left) and blank (right) environment. (E) Same as on (D) when comparing V2 LFP with the LFP of the motor cortex, serving as a control. (F) Comparison of the maximal coherence values between 40-55 Hz and 5-50 ms. Left and right panel compares the coherences of the motor cortex vs. V2, and CPu vs. V2, respectively, during running in striped and blank environments. 


\section{DISCUSSION}

The main conclusion of my work is that in addition to the small distinct group of striatal neurons that are responsive to both self-motion independent (passive) and self-motion induced (active) visual stimuli, there is a larger subset of striatal cells that only sense self-motion induced visual changes. Considering this new information, it is essential to investigate visual motion-related sensory processing in freely moving animals, instead of in anesthetized or headfixed preparations. The perceptual capacity represented by these two cell groups is likely essential for survival, as they allow for recognition of incongruently moving objects (such as prey) in a visual environment, which is continuously changing by itself due to the self-motion of the observer. Although the two functional groups share many electrophysiological properties, our results suggest that $\mathrm{pVis}+$ neurons were overwhelmingly FFNs.

\subsection{Considerations of striatal cell type identification from extracellular recordings}

The anatomical group of MSNs are traditionally identified electrophysiologically as PFNs; however, this group is not homogenous, neither regarding its receptor-repertoire and connectivity (D1 and D2 neurons, Chuhma et al., 2011) nor on its behavioral correlates (Hernandez et al., 2013). A small, but still well-characterized subset of the non-MSN group is cholinergic. They elicit both nicotinergic excitation and post-synaptic neuromodulation (Zhou, Wilson and Dani, 2002), and as they maintain a relatively stable firing rate and rarely burst, they are traditionally identified electrophysiologically as TFNs (Aosaki et al., 1994). The rest of the $\mathrm{CPu}$ neurons are aspiny GABAergic interneurons. This ambiguous, heterogenic group may play an orchestrating role in shaping striatal function since they express an extensive influence on the rest of the network (Sharott et al., 2009). Observing the function of aspiny GABAergic interneurons in awake animals would first require establishing a reliable classification criteria from extracellular spike trains. This would require the use of optogenetic tagging in behaving animals, as the easy-to-measure features (e.g., spike-width, burstiness, etc.) overlap with other cell types, and their behavioral responses vary with specific innervations of the given striatal region. Cross-correlation analysis of monosynaptically or electrically coupled neuron pairs may also give a hint towards cell identity (spike transmission efficacy, excitation/inhibition), but reliable quantification of excitatory transmission requires many hours of continuous recording, especially if either one of the cells is low-firing (Schwindel et al., 2014). 
We were able to isolate significantly fewer PFNs and more FFNs in our recordings (similarly to Thorn and Graybiel, (2014)) as was expected from histological studies. While intracellular and patch-clamp recordings provide a uniform unbiased sampling (or at least one not influenced by electrophysiological properties of the neurons), the voltage traces of neurons with very low firing rates are frequently either discarded during the classification process or excluded from the analyses, due to numerical approximation issues. PFNs are particularly sparsely firing neurons; thus they are the most vulnerable to false-exclusion or skipped detection. In contrast, all well detectable fast and irregular firing neurons are uniformly classified as FFNs, although this feature is confirmed only for parvalbumin-positive fastspiking interneurons (FSIs) (Mallet et al., 2005; Berke, 2008), not for the rest of the GABAergic aspiny neuron classes: The various types of the tyrosine-hydroxylase positive (but still GABAergic) neurons cannot be reliably distinguished from FSIs by their electrophysiological properties (Tepper et al., 2010; Ibanez-Sandoval et al., 2011), even if their connectivity is strikingly different. However, there is solid evidence that persistent low-threshold spike (PLTS)/low-threshold spike (LTS) neurons generate low-threshold calcium bursts (Sharott et al., 2009). We could not identify such bursts in any neurons of the FFN group at all; thus it is not likely that these groups contaminate it.

Observing the function of the classes distinguished in slice recordings in awake animals would first require establishing reliable classification criteria from extracellular spike trains, for example, with the help of optogenetic tagging. Unfortunately, most of their easy-to-measure features (e.g., spike-width, burstiness, etc.) are overlapping; thus, the higher number of classes one wants to distinguish, the more features to be investigated simultaneously. This extensive testing (recording during multiple behavioral states, e.g., resting, locomotion, still alertness, sleep, etc.) is rarely feasible due to technical limitations of obtaining stable recording quality of the same neurons for the duration of the testing sequence, or the finite capacity of the animals to cooperate. Another aspect of functional heterogeneity is that the responses of a given class to behavioral tests (e.g., auditory, visual stimulation, reward-related activity) and their network interactions (e.g., phase coupling to LFP oscillations) may also depend on the intraCPu location of the cells, since their topographical cortical afferentation may be substantially different. Cross-correlation analysis of monosynaptically or electrically coupled neuron pairs may also give hints on the identities (spike transmission efficacy, excitation/inhibition), but this approach 
faces the same bottleneck; reliable quantification of these measures requires many hours of continuous recording, especially if either one of the cells are a low-firing one (Schwindel et al., 2014). In most cases, these technical challenges make the identification unfeasible; future advances in multimodal optical identification (e.g., expressing multiple optoproteins simultaneously, each being selective for a given cell type, and excitable only with a narrow bandwidth of light) might allow in vivo screening relatively quickly, and refine results obtained from extracellular recordings.

\subsection{Immediate Early Genes (IEGs) in the CPu}

Conclusions about the visual responsivity of striatal neurons based on the results of the cFos expression experiments alone should be interpreted with caution. Although the $\mathrm{CPu}$ is known to express IEGs in response to cortical stimulation (Berretta, Parthasarathy and Graybiel, 1997), they are not uniformly expressed among different cell types, only increases of firing rates can be reliably described (Hughes and Dragunow, 1995) and lastly, it is not possible to distinguish mono- and polysynaptic activation. We chose to detect cFos over JunB, FosB or other IEGs, because of its very low constitutive expression in the $\mathrm{CPu}$, and because most cell lines express it upon stimulation (Berretta, Parthasarathy and Graybiel, 1997).

The $\mathrm{pVis}+$ neurons were inhibited by visual motion, which cannot explain the induced cFos expression. We speculate that it was either induced by the blinking light or the stationary grating parts of the stimulation sequence (i.e., more than half of the $\mathrm{pVis}+$ neurons were excited by the change of brightness, and they were also typically excited by the stationary gratings). An alternative explanation is that these neurons were activated through disinhibition, as the $\mathrm{pVis}+$ neurons were mainly FFNs, but the cFos + cells were PV-. In this latter case, the cFos + cells could be mainly medium spiny neurons, which are known to receive inhibitory innervation from the FFNs. Future studies may reveal the exact identity of these neurons (e.g., distinguishing between direct and indirect pathway MSNs by staining for dynorphin and encephalin, respectively, (Graybiel, 1990)), and transsynaptic tracing techniques may reveal if they receive direct synaptic inputs through the BDA-labelled cortical axons. 


\subsection{Monosynaptic interactions}

Although the FSIs provide powerful perisomatic inhibition to surrounding MSNs in slice (Koós and Tepper, 1999), we only observed a few examples of this interaction (1\% of the theoretically possible FFN-PFN connections; see also Berke (2011)). FSIs and neurogliaform cells are known to connect with peers from the same class through electrical synapses; however, the functional role of such a syncytium is debated. Network models of FSI neurons suggest that these gap junctions instead serve as shunts when sporadic inputs reach the interconnected network, but have negligible effect when inputs are synchronous (Hjorth, Blackwell and Kotaleski, 2009). We found a high number of synchronous excitatory FFN pairs $(6.77 \%$ of the maximum possible), but in most cases, the height of the zero time-lagged CCG peaks showed less than ten percent synaptic transmission efficacy, and not an all-or-none coupling. Experiments on dopamine-depleted animals further controvert this strong coupling by showing that striatal L-DOPA treatment normalized the firing rate of projection neurons, while the elevated activity of the FSIs was maintained (Hernandez et al., 2013). These findings oppose the speculation that a high level of synchrony among FFNs boosted by high spontaneous firing rates induces a potent inhibition on their targets.

We couldn't detect inhibitory synapses between PFN-PFN and PFN-FFN pairs, which confirms the earlier finding that MSN-MSN interactions are weaker, because they target distal dendrites (Koos, Tepper and Wilson, 2004), and that MSNs do not reciprocally innervate FSIs (Chuhma et al., 2011). In contrast, cholinergic TFNs could evoke monosynaptic activation of FFNs, but not PFNs, which is in agreement with the earlier studies showing that FSIs can be excited by nicotinic agonists (Luo et al., 2013). Recent experiments suggest that TFNs can induce a massive GABAergic inhibition on MSNs, either through FFNs or through co-release from nigrostriatal dopaminergic terminals (Nelson et al., 2014).

Altogether, these findings support the concept that the role of gap junctions are to build an 'input filter', which is sensitive to synchronized cortical inputs, rather than to provide a universal 'brake' (Hjorth, Blackwell and Kotaleski, 2009). However, the few excitatory connections we found between PFNs and FFNs or TFNs, which contradict the known transmitter/receptor repertoire of the given neurons, are stressing out the limitations of CCG analysis on low spiking cell pairs. 


\subsection{Origin and processing of the visual information in the dorsomedial striatum}

Hunnicutt et al. (2016) reported that neurons of the dorsomedial part of the mouse striatum respond to multiple sensory modalities, and they integrate auditory and visual inputs with emotional and contextual information arriving from the amygdala and the hippocampal formation, respectively. We found that aVis + neurons were sensitive to global (but not local) luminance changes. This finding is in agreement with earlier reports on the large receptive fields of striatal cells spanning almost the whole visual field in cats (Gombkötö et al., 2011). These results suggest that the striatum receives a preprocessed 'global percept' of the visual environment, instead of a partial pixelated representation. We found that a possible source of this information may be the visual cortex, which is supported by earlier reports on a direct connection between the visual cortex and dorsocaudal striatum in rats (Serizawa et al., 1994; Khibnik, Tritsch and Sabatini, 2014). This glutamatergic pathway was found to innervate the matrix but not the striosomes selectively, and to terminate mainly on MSNs (Khibnik, Tritsch and Sabatini, 2014; Hunnicutt et al., 2016). This is in agreement with the putatively PFN nature of the neurons monosynaptically excited by electrical stimulation in the current study; however, it cannot explain why most of the pVis + neurons were FFNs.

FFNs also receive direct cortical inputs, but due to their high spontaneous firing rates, it is harder to detect an induced excitation, even if they are otherwise more sensitive to cortical inputs than MSNs (Berke, 2011). The inhibition of the FFNs exerted by visual stimuli suggests either an indirect cortical effect through other striatal interneurons (signal inversion) or an already negative coding scheme of the cortico-striatal afferents. This latter scenario may be realized through the complex neurons of the $\mathrm{V} 1$, and the so-called ' $\mathrm{D}$ ' cells of the extrastriate cortex with maintained spontaneous firing rates, which are decreased when visual motion is perceived (Gizzi et al., 1990). Such a decrease in the cortical drive may explain the motioninduced decline of spiking in the $\mathrm{pVis}+$ neurons.

An earlier report (Nagy et al., 2010), found that caudate nucleus neurons of the cat prefer similar low spatial and high temporal frequencies to what we report here regarding the $\mathrm{pVis}+$ neurons, which suggests a conserved tuning across species. Regarding its afferents, the anterolateral part of area 18a (lateral extrastriate cortex) of the mouse, which is the homolog of V2L and V2LM in the rat (Sereno and Allmann, 1991), is linked to the processing of self- 
motion cues (Andermann et al., 2011), and also expresses similar spatiotemporal sensitivity. Besides, Montero and Jian, (1995) showed that while cFos expression could be induced in the $\mathrm{V} 1$ and the anteromedial and lateromedial part of area 18 by both stationary and moving scenes, LM was only sensitive to moving stimuli. Seventy-five percent of the V2 neurons we recorded were modulated by the stripedness of the linear maze, similar to the aVis $+\mathrm{CPu}$ neurons. These cells did not respond to blinking light stimulation at all (also found by Berke, (2008)), which suggests that the visual information may originate from a higher-order visual structure that is not sensitive to primitive on-off luminance transitions.

The various features of visual information serving motion perception and sensorimotor feedback, seem to be rather distributed functionally also. Lesioning V1 impairs orientation discrimination and optic flow perception in rats; however, approaching salient visual targets is surprisingly intact in these animals. V2 lesions cause no such impairment, or any behavioral deficit at all (Petruno, Clark and Reinagel, 2013), which raises the possibility of a non-cortical mechanism for integrating the sensory percept with the motor behavior.

One possible subcortical pathway that can convey representation of the visual world to the $\mathrm{CPu}$ originates in the lateral-posteromedial nucleus of the thalamus, which projects to the same subregions of both in the $\mathrm{CPu}$ and the V2 that we investigated here (Kamishina et al., 2008; Rubio-Garrido et al., 2009). This region may play an essential supplemental role in the visual representation of context (Roth et al., 2015), however, its effect on the $\mathrm{CPu}$ is yet unknown. An alternative candidate is a retina - superior colliculus - intralaminar thalamus $\mathrm{CPu}$ pathway (Coizet, Overton and Redgrave, 2007), which converges with tectonigral dopaminergic signals to enhance the detection of unpredictable salient signals (Redgrave, Prescott and Gurney, 1999). Our finding that almost all pVis + neurons were excitable by electrical stimulation of the V2 in our current study, strengthened by the gamma-band coherence of these structures that is modulated by the visual percept, suggests that a major source of the visual information in the $\mathrm{CPu}$ is rather cortical, but the lack of electrical excitability of aVis + neurons also predicts a complementary subcortical input. 
The significantly higher number and more uniform intrastriatal distribution of the aVis + neurons compared to the less frequent, and spatially restricted $p V i s+$ cells offers the idea of multiple parallel visual streams, whose recipients are selectively responsible for self-induced and self-independent motion perception respectively, and the different behavioral and cortical states during the $\mathrm{pVis}$ and the aVis tasks may also bias the properties of the two cell groups (McGinley et al., 2015). These concepts are only partially supported by our results, though; the overwhelming presence of FFNs in both groups, the similar response profile in the striped maze, the major overlap between the two groups, and other investigated properties, propose that the pVis + neurons are more likely a subtype of the aVis + group. Given that the aVis + neurons located at the more ventral and lateral parts of the $\mathrm{CPu}$ do not receive direct V2 input, they cannot be entrained by electrical stimulation, and do not express cFos during passive visual stimulation; they are likely to receive their visual innervation differently. The route may either be the aforementioned subcortical pathways, or an intrastriatal indirect pathway driven by cortical inputs, which target the dorsomedial $\mathrm{CPu}$.

\subsection{Multimodal integration in the dorsomedial striatum - Network level oscillations}

Interactions between multiple circuits of the brain are orchestrated by coherent LFP oscillations serving as 'semaphores', which are efficient filters for information transfer (Buzsaki, 2004b). We found that the neurons of V2 express oscillations in the mid-gamma band (called gamma-50 by van der Meer and Redish, (2009)). Such patterns also appear in the CPu with approx. 20-30 ms delay, which we interpret as a sign of information transfer between the visual cortex and the striatum. This delayed synchrony is not present at other frequency bands (except for theta band), and it quickly decays even if the signals are further lagged with delays matching an integer number of cycles, which suggests that the detected events are discrete packages of a few gamma cycles, and not a persistent gamma oscillation. The lag of this gamma epoch is in good agreement with the latency of the striatal EPSPs compared to the V1 evoked potentials after visual stimulation reported by Reig and Silberberg, (2014) and matches the delay of the monosynaptic responses recorded in our electrical stimulation experiment. It is possible that its purpose is to offer highly excitable time-slots when the $\mathrm{CPu}$ neurons become more sensitive to cortical information; however, it is doubtful whether these gamma patterns are related only to the visual cortical interactions, or multiple inputs are converging to the $\mathrm{CPu}$ during these short temporal windows. 
We assume that these gamma events in the $\mathrm{CPu}$ are likely to be generated by the evoked gamma frequency bursts of FSIs (Schulz et al., 2011), since similar PV+ interneurons are responsible for gamma oscillations in the hippocampus and cortex (Klausberger et al., 2003). This hypothesis is compatible with our finding that the majority of $\mathrm{pVis}+$ neurons are FFNs, underpinning that their modulation through sensory inputs may affect the generated gamma events. Visual stimulation decreases the firing rate of the afferenting visual cortical neurons and the FFNs; thus, we assume that this decreased cortical 'driving constrains' allows for a more independent gamma generation, which can explain the weaker gamma coherence in the striped maze. Similarly, the weak theta phase coupling reflects the relatively steady responsivity of the $\mathrm{pVis}+$ neurons, which is a prerequisite for the reliable, quick recognition of moving objects.

The mechanistic understanding of the generation and effect of the various LFP oscillations detectable within the $\mathrm{CPu}$ are unclear, mainly because of the weak phase coupling and the lack of any ordered geometrical alignment of the striatal neurons. Computational models and observations in Parkinson's disease suggest that the $\mathrm{CPu}$ can generate beta-band oscillation on its own, through the interplay between FSIs and MSNs under the control of dopamine neuromodulation (Kondabolu et al., 2016). The picture is not so evident in the case of gamma oscillations. Van der Meer et al., 2010, found that at least part of the ventral striatal gamma activity is locally generated; however, the $\mathrm{CPu}$ network lacks the necessary inhibitoryexcitatory loop that could generate these rhythms alone (Bartos, Vida and Jonas, 2007; Fries, Nikolić and Singer, 2007). Along these lines, rhythmicity is likely to be generated elsewhere (e.g., visual and cortices or hippocampal formation), and the striatal neurons only resonate with these inputs, and resemble the oscillations. The sudden switches between 50 and $80 \mathrm{~Hz}$ gamma rhythms along the whole extent of the ventral striatum still suggests the presence of coherent internal mechanisms (van der Meer and Redish, 2009).

Although theta power in the striatal LFP spectra is very strong, and theta related neuronal features such as theta phase precession is present in a good number of neurons, in vitro studies could not reveal any cellular properties that may support the local generation of the theta rhythms (Taverna, Canciani and Pennartz, 2007). Since this phase precession has less uncertainty if calculated using the phases of hippocampal theta rhythm instead of local phase 
values (van der Meer and Redish, 2011), CPu likely receives and resembles a theta modulated activity from the hippocampus or connected structures (e.g., prefrontal cortex), (Jones and Wilson, 2005; Siapas, Lubenov and Wilson, 2005), rather than generating the rhythm by itself. Similarly, delta oscillations are imposed by corticostriatal neurons driving striatal interneurons and MSNs (Wilson and Kawaguchi, 1996; Mallet et al., 2005). The proxy and local generation of lower and higher frequency oscillations, respectively is reinforced by the observation that selective activation of TFNs selectively increases the power of the beta and gamma bands, but not the lower frequency bands (Kondabolu et al., 2016). The solid phase locking of the recorded neurons to gamma oscillations in this study also supports this idea. Along the same lines, the decoupling of FFNs from theta oscillation in response to visual stimulation may be at least partially explained if these neurons are not taking part in the theta generation per se.

\subsection{Multimodal integration in the dorsomedial striatum - Processing non-sensory modalities}

Despite the remarkable topographic organization of the striatal afferentation, its functionality is not strictly separated by sharp borders, but rather forms overlapping gradients (van der Meer et al., 2010). The dorsomedial part is considered mainly as an associative zone, serving multisensory integration (Thorn and Graybiel, 2014). This segment of the CPu receives a broad repertoire of sensory modalities, as well as other sources reflecting the internal dynamics of the brain (Reig and Silberberg, 2014; Wilson, 2014).

As the animals were running in the linear track, we found a substantial number of neurons possessing place field-like activity. A few of them showed theta phase precession as well. This is more likely a projection of the intrahippocampal phase precession and not a locally, de novo generated feature since it is more strongly coupled to the hippocampal theta phases than to the striatal theta. Nonetheless, this latter can also, at least partially, be a volume conducted oscillation reflecting the hippocampal theta, as they are highly coherent, but striatal theta is also modulated by, e.g., reward signals to some extent (van der Meer and Redish, 2011). While the phase precession of the ventral striatal 'ramp' neurons is determined by their massive innervation through the fimbria (van der Meer and Redish, 2011), the mediodorsal CPu also receives hippocampal innervation through at least four pathways that can explain its behavior. The subiculum innervates the medial and caudal part of the $\mathrm{CPu}$, either directly or through the entorhinal cortex, and there are also indirect connections through the medial prefrontal cortex 
and the posterior cingulate cortex (Devan and White, 1999). The robust phase shifting of the precessing neurons, in relation to the hippocampal theta, suggests that it is more likely the former, more direct pathways convey the phase precession.

This afferentation may also explain the presence of 'place field' like behavior (O'Keefe and Dostrovsky, 1971) found in many $\mathrm{CPu}$ neurons. The experimental settings of the current study cannot reliably differentiate if the recorded firing pattern is purely related to a spatial location, or if reward prediction also shapes them as the animals are approaching the water ports (van der Meer and Redish, 2009). Nevertheless, the simultaneous presence of a visual contextdependent and independent activity (i.e., the presence of the striped pattern did not alter the spatial location of the place fields, but could modulate the intra-place field firing rates) also points out the integrative nature of the neuronal activity patterns in the striatum. In agreement with the findings of van der Meer and Redish, (2009), medium gamma (gamma-50) power expressed a substantial increase upon the reception of the reward, while high gamma (gamma80) showed an abrupt decline after a gradual buildup. We observed similar properties regarding an increased beta power, and a drastic drop in theta rhythm. The latter may be explained by the known linear relation of running speed and theta power in the hippocampal formation (Geisler et al., 2007), however, reaching reward, even when the exploring animal was not running, can result in a drop in theta power (Horst and Laubach, 2013).

The reward-related activity of the $\mathrm{CPu}$ is an integral part of the hippocampal, anteromedial prefrontal cortex, and the dopaminergic subcortical reward systems. The interaction of these inputs with the sensorimotor components provides a plethora of information to associate places with reward, and learn optimal survival strategies (van der Meer and Redish, 2011). Traditionally, the hippocampus and the striatum were considered as two independent but complementary memory systems, responsible for episodic and procedural memory, respectively (DeCoteau et al., 2007). An alternative concept is that the CPu should be better considered as an integrator that has the chance to adjust procedural motor functions based on the internal representations of 'past experiences' (i.e., through hippocampal episodic and spatial memory, (Akhlaghpour et al., 2016)) and the 'present circumstances' (i.e., through the contribution of sensory systems). Our findings regarding the coexistence and simultaneous, conjunctive expression of sensory-related and contextual/spatial (e.g., hippocampal) features in the $\mathrm{CPu}$ may support this latter concept. 


\section{CONCLUSIONS}

Conclusively, differential innervation distinguishes $\mathrm{pVis}+$ cells from the larger pool of self-motion induced visual perceptors (i.e., aVis + neurons) and establishes them as a separate functional group. We propose that the aVis+ neurons may provide robust feedback on the dislocation of the gait caused by the movements of the animal, while the successive comparison of the activity of aVis + and $\mathrm{pVis}+$ neurons can reveal the presence of independently moving third-party objects such as prey or predators in a non-stationary but neutral visual environment. This process may be fundamental in detecting self-independent moving objects and selecting the appropriate action to challenge them. Further experiments are needed to gain evidence and verify these hypotheses; however, it is clear from our current results that such sophisticated distinctions can only be made if experiments are carried out on freely moving animals, where the congruency of the changes in the visual environment can be arbitrarily decoupled from the other modalities. Anesthetized or head-restrained arrangements or even passive visual stimulation of freely moving animals are bound to do a poor job mapping the differences in self-motion induced and self-independent visual perception. 


\section{MATERIALS AND METHODS}

All experiments were approved by the Ethical Committee for Animal Research at the Albert Szent-Györgyi Medical and Pharmaceutical Center of University of Szeged (No. XIV/218/2016 and XIV/471/2012). 32 male rats (Long-Evans, 3-12 months old) were used in this study.

\subsection{Development of multiplexing data acquisition hardware}

\section{Signal multiplexing and processing}

A major challenge of the effective use of silicon probes in small animals is to reduce the volume and weight of the instrumentation between the probe and the recording equipment. Multiple connectors, traditional preamplifiers, and multi-strand cables for each recording site are not viable for high-density probes; the large volume and weight of these components and the increased tension of the connecting cable significantly limit the behavior of the small rodent. A logical solution to these problems is the deployment of time-division multiplexing (Olsson 3rd et al., 2005; Harrison, 2008; Szuts et al., 2011; Viventi et al., 2011). To this end, we used 32 channel VLSI analog signal multiplexers explicitly developed for brain recording applications (Harrison, 2008) (RHA2132, INTAN Inc., www.intantech.com). Multiplexers are soldered to a custom-designed lightweight printed circuit board (Fig. 9A) to transmit up to 32, 64 , or 256 channels. To allow movement of the probe and to preserve its small inertia, an ultraflexible polyimide-based cable was designed and served as an interconnect between the probe and the multiplexing headstage (Fig. 9A).

Each of the electrical signals acquired by the silicon probe is amplified and band-pass filtered (gain $=200 \times$, cutoff frequencies of the high-pass and low-pass filters are $0.3 \mathrm{~Hz}$, $-6 \mathrm{~dB} /$ octave and $10 \mathrm{kHz},-18 \mathrm{~dB} /$ octave, respectively) (Fig 9B). The broadly tuned cutoff frequencies allow the recording of broad-band signals (LFP and unit activity simultaneously) from all recording sites. The signal multiplexer chips were programmed to switch sequentially between channels every $1.5 \mu \mathrm{s}$, so that a sweep of 32 channels corresponds to $48 \mu \mathrm{s}$ (Fig 9C, D). To accomplish a $50 \mu$ s cycle length ( 20 complete scan cycles in a millisecond), the last channel of the 32 channel block was transmitted for two more microseconds before looping back to the first channel of the subsequent cycle. The post-loop $2 \mu$ s time was used to protect against the desynchronization of the multiplexing sequence. Potential desynchronization of the multiplexing and demultiplexing sequence may occur if the clock signal accidentally advances 
the sequence of the multiplexed signal. Such accidental 'jumps' are avoided by resetting the binary counters to zero (binary code 00000) during the $2 \mu$ s post-loop time. This clock supervision mechanism ensured that even if the clock code is corrupted for any reason, only one sample is misplaced in the sequence of the parallel-decoded channels.

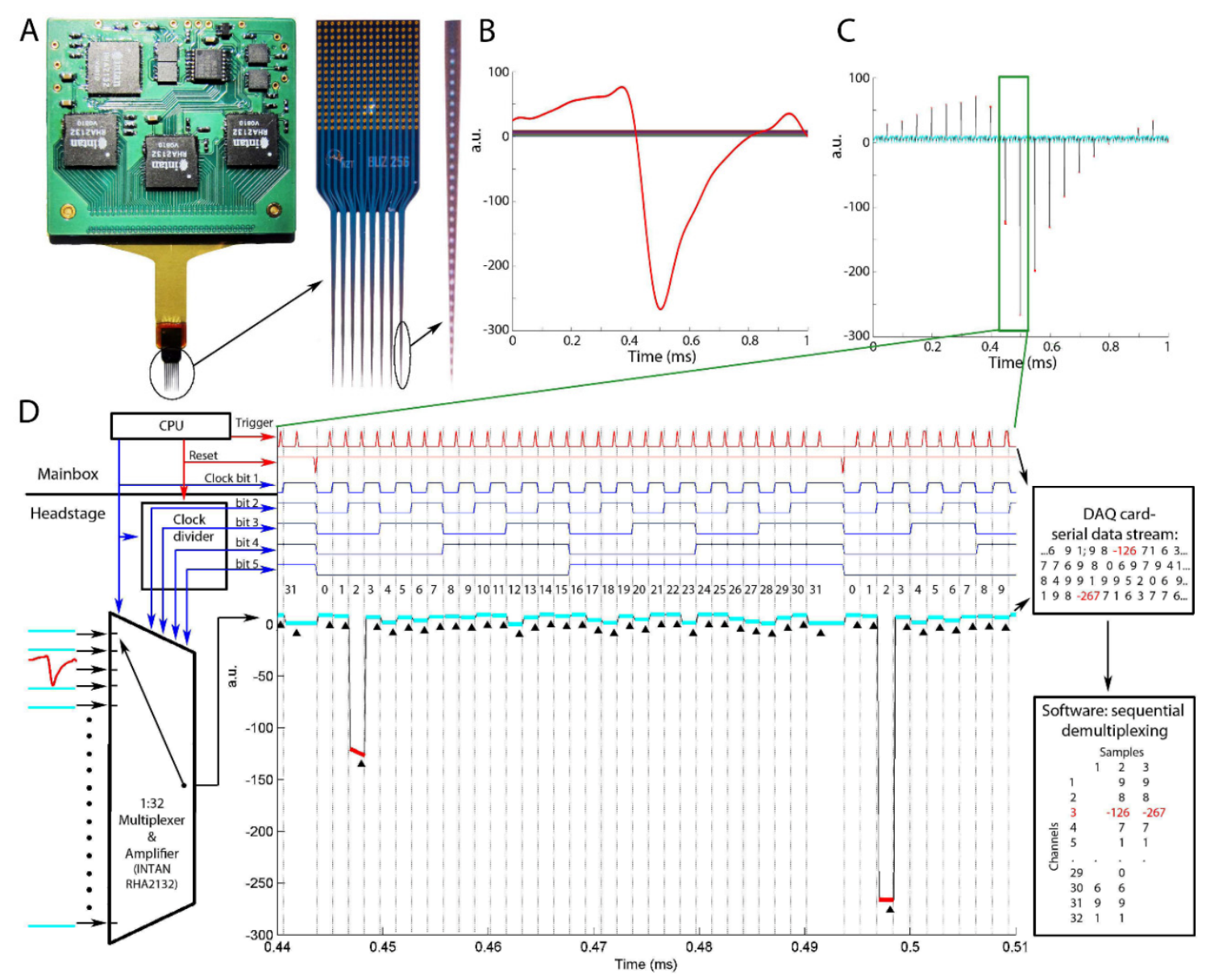

Figure 9. System overview. (A) High-channel-count (256 sites) silicon probe connected to a printed circuit board via a flexible polyimide ribbon cable. The printed circuit board contains eight separate 32-channel signal multiplexers (4 on each side) and accessory circuit elements. (B) A simulated headstage input signal is illustrating a spike waveform on 1 of the 32 channels and various levels of direct current (DC) on the remaining 31 channels (1 ms). a.u., Arbitrary units. (C) Time-shared multiplexed signal transmitting the 32 channels shown in B. (D) Circuit schematics and working principle of the multiplexer and a zoomed segment of the time epoch indicated by a rectangle in $\mathrm{C}$. CPU, central processing unit.

We found that the relatively high output impedance of the RHA-2132 multiplexer chip was not sufficient to reliably transmit signals over long data cables (over 5 meters), typically needed in behaving experiments. To improve signal settling time and to circumvent the 
capacitive load of the cable, high precision operation amplifiers (MCP622; Microchip, USA; slew rate: $27 \mathrm{~V} / \mu \mathrm{s}$ ) were added in series to buffer the multiplexed signals. With the added buffering, the signal settled in less than $100 \mathrm{~ns}$ to $1 \%$ precision after each switch of the multiplexer chip even when up to 15 meter-long cable, consisting of 36-gauge (110 $\mu \mathrm{m})$ Litz wires, was used. The ultra-light and ultra-flexible cable allowed for a free movement of the animals (Vandecasteele et al., 2012).

To achieve synchronous channel advancement on every multiplexer chip without adding further weight and surface area to the head stage, the common clock signal $(640 \mathrm{kHz}, 50 \%$ duty cycle) is generated by a programmable integrated circuit in an external controller box ('main box'). A five-bit binary clock code was generated by a synchronous binary counter (74HC163; Texas Instruments, USA) on the head stage to address the channels of the multiplexers within blocks of 32. In the main box, the multiplexed signals were further amplified (gain $=2$, total gain $=400 \times$ ), and their baseline was corrected by the subtraction of a preset DC value. The multiplexed signals (Fig. 9C, D) were sampled by a high-speed digital-to-analog converter (PCI-6133; National Instruments, USA). Sampling was initiated by the main box's clock generator microchip $125 \mathrm{~ns}$ before the subsequent channel switch to exploit the available signal settling time fully. The electrophysiological recording setup was combined with a universal serial bus (USB) web camera recording system for the continuous monitoring of the animal's position and behavior. The system was also furnished with a custom-designed analog demultiplexing circuitry, which provided real-time access (lag $<50 \mu \mathrm{s})$ to any selected electrode signal for closed-loop applications and feedback purposes.

\section{Software control}

To generate the clock and trigger signals, the programmable integrated circuit was activated by the recording software only after the digital-analog data acquisition (daq) task was properly set up, and was idle for the external trigger to start sampling. The recording software program acquired samples from the buffer in chunks of $100 \mathrm{~ms}(100 \mathrm{~ms} \times 20$ samples per ms $\times$ 32 channel $=64000$ samples per multiplexed line $)$. This $100 \mathrm{~ms}(10 \mathrm{~Hz})$ readout frequency offered a practical compromise between minimizing the time requirement introduced by the frequent updates of the processing code headers, yet provided a smooth, real-time-like data display on the computer screen. After the buffer has been filled, the acquired data was read out and was reordered to a $32 \times 2000$ size matrix (Fig. 9D). 


\section{Physical dimensions}

During recording, the animal was connected to the recording system through an ultraflexible cable (Vandecasteele et al., 2012) (twelve $127 \mu \mathrm{m}$ diameter insulated copper wires, $455 \mu \mathrm{m}$ total diameter cable). For recording from smaller size silicon probes or tetrodes targeting multiple brain structures, we constructed 32 and 64-channel versions of the multiplexer headstage (32-channel 0.9 gram; $13 \mathrm{~mm} \times 15 \mathrm{~mm} \times 3 \mathrm{~mm}$; 64-channel: 1.2 gram; $13 \mathrm{~mm} \times 19 \mathrm{~mm} \times 3 \mathrm{~mm}$, respectively). These headstages were connected to the probe output by high-density connectors (Omnetics, Inc.) during the experiments. We also designed two versions of a 256-channel multiplexer headstages. One of them was permanently connected to the 256-channel silicon probe and was worn chronically by the rat ( 4.7 grams; $34 \mathrm{~mm} \times 39 \mathrm{~mm}$ $\times 2.5 \mathrm{~mm}$ ), while the other one was detachable in a 'sandwich' fashion, using high-density Panasonic connectors ( 3 grams; $19 \mathrm{~mm} \times 34 \mathrm{~mm} \times 3 \mathrm{~mm}$ ).

\subsection{Anatomical tract tracing experiments}

Long-Evans rats $(260-580 \mathrm{~g})$ of both sexes were anesthetized with 1-3\% isoflurane and then mounted on a stereotaxic apparatus. Atropine $(0.1 \mathrm{mg} / \mathrm{kg}$, s.c.) was administered immediately after the anesthesia induction. Stages of anesthesia were maintained by confirming the lack of vibrissal movements and nociceptive reflex. The rectal temperature was maintained at $35-37{ }^{\circ} \mathrm{C}$ (TMP-5b; Supertech, Pecs, Hungary). A small incision on the skin was then made for craniotomy after subcutaneous lidocaine injections.

\section{Anterograde tract-tracing of corticostriatal neurons}

A craniotomy above the right secondary visual cortex (V2) was made $4.0-5.0 \mathrm{~mm}$ posterior from the bregma, 2.0-3.0 mm lateral of the midline (Paxinos and Watson, 2013). 10\% biotinylated dextran amine (BDA) (D1956; Thermo Fisher Scientific, Waltham, MA) in $0.1 \mathrm{M}$ phosphate-buffered saline (PBS) was injected into the V2 according to the following four stereotaxic coordinates: (in mm) AP -4.50, ML 2.25; AP -4.50, ML 2.75; AP -5.00, ML 2.25; AP -5.5, ML 2.25. using an auto-nanoliter injector (Nanoject II; Drummond Scientific, Broomall, PA). For each coordinate, $0.3 \mu \mathrm{l}$ of the BDA solution was ejected at $0.7,0.85$, and $1 \mathrm{~mm}$ below the pia at a rate of $0.09 \mathrm{nl} / \mathrm{s}$. After ejections, the capillary tip was held in the position for at least five minutes and then gently retracted. The craniotomy was covered with a sterile bone wax, and the skin wound was sutured. After one-week of survival, the rats were deeply anesthetized, perfused, and the brains were processed (see below). 


\section{Retrograde labeling of striatal afferents}

Four Long-Evans rats were anesthetized and then mounted on a stereotaxic apparatus as described above. A craniotomy above the striatum $(\mathrm{CPu})$ was made. A glass capillary filled with $4 \%$ fluorogold (Fluoro-Gold; Fluorochrome, Denver, CO) in $0.9 \%$ saline was installed with an auto-nanoliter injector (Nanoject II) and then inserted into the caudate-putamen according to the following coordinate: (in mm) AP $-1.25-1.75$, ML 3.6-4.0, and DV 3-4 from the Bregma, midline, and pia, respectively. For each coordinate, $0.15-0.30 \mu 1$ of the solution was gently ejected. After one-week survival, the rats were transcardially perfused, fixed, $60 \mu \mathrm{m}$-thick coronal brain sections were prepared, and processed as described below.

\section{Tracing experiments in combination with visual stimulation}

Nine rats underwent the BDA injection of the anterograde tracing, as described above. After one-week of survival, the rats were reared in complete darkness overnight, and then five of them were exposed to the same passive visual stimulation used in the freely moving electrophysiological experiments (see below). The four other animals were left in the darkness for the same duration. After that, the rats were immediately anesthetized, perfused, and their brains were processed for cFos and BDA immunohistochemistry as described below. In one animal, we also tested the identity of cFos + cells with immunohistochemistry against parvalbumin.

\subsection{Tissue processing and immunohistochemistry}

Following the termination of the experiments, animals were deeply anesthetized. All animals were transcardially perfused first with $0.9 \%$ saline solution followed by $4 \%$ paraformaldehyde solution. Brains were sectioned in 50-70 $\mu \mathrm{m}$ thick coronal slices (VT1000S Vibratome). The following staining procedures were performed at room temperature.

\section{Immunohistochemistry for retrograde tracing}

Sections were incubated successively with $10 \%$ NGS in PBS-X for $30 \mathrm{~min}, 1: 2000$ diluted anti-fluorogold polyclonal antibody (AB153-I; EMD Millipore, Billerica MA) in PBS-XG for $48 \mathrm{~h}$, and $4 \mu \mathrm{g} / \mathrm{ml}$ Alexa Fluor 633-conjugated goat anti-rabbit IgG (A-21071; Thermo Fisher Scientific) in PBS-XG for $2 \mathrm{~h}$. Sections were counter-stained with a fluoro-Nissl solution for $60 \mathrm{~min}$, washed with PBS, mounted and coverslipped. 
Immunohistochemistry for the anterograde tracing combined with visual stimulation

Animals were transcardially perfused with physiological saline followed by $4 \%$ paraformaldehyde (PFA) and $0.2 \%$ picric acid in $0.1 \mathrm{M}$ phosphate buffer $(\mathrm{PB})(\mathrm{pH} 7.2-7.3)$. After removal, brains were postfixed overnight, embedded in $4 \%$ agarose, coronally sectioned at $50 \mu \mathrm{m}$-thick using a vibrating blade microtome, and harvested in PBS. All incubations were followed by washing with PBS containing $0.3 \%$ Triton X-100 (PBS-X). Sections were incubated successively with $10 \%$ normal goat serum (NGS) in PBS-X for $30 \mathrm{~min}, 1 \mu \mathrm{g} / \mathrm{ml}$ rabbit anti-cFos polyclonal antibody (ABE457; EMD Millipore) (Kim et al., 2014) in PBS-X containing $1 \%$ NGS and $0.02 \%$ sodium azide (PBS-XG) overnight, and a mixture solution containing $4 \mu \mathrm{g} / \mathrm{ml}$ Alexa Fluor 488-conjugated goat anti-rabbit IgG (A-11034; Thermo Fisher Scientific) and $2 \mu \mathrm{g} / \mathrm{ml}$ Alexa Fluor 555-conjugated streptavidin (S-21381; Thermo Fisher Scientific) in PBS-XG for $2 \mathrm{~h}$. Sections were then mounted on glass slides, counter-stained with a fluoro-Nissl solution (N-21479; Thermo Fisher Scientific) for $40 \mathrm{~min}$, washed with PBS, and finally coverslipped with $50 \%$ glycerol and $2.5 \%$ triethylene diamine in PBS (Takeuchi et al., 2017).

cFos and parvalbumin (PV) double immunohistochemistry

Sections were incubated successively with $3 \%$ bovine serum albumin (BSA) in PBS-X for 30 min, a mixture of 1st antibodies (rabbit anti-cFos at 1:000: ABE457; EMD Millipore and mouse anti-PV at 1:3000: PV235; Swant, Marly, Switzerland) in PBS-X containing 1\% BSA (PBS-XBSA) overnight, and a mixture solution containing $4 \mu \mathrm{g} / \mathrm{ml}$ Alexa Fluor 488-conjugated goat anti-rabbit IgG and $4 \mu \mathrm{g} / \mathrm{ml}$ Alexa Fluor 594-donkey anti-mouse IgG (A-21203; Thermo Fisher Scientific) in PBS-XBSA for $2 \mathrm{~h}$. Sections were then mounted, counterstained, and coverslipped.

\section{Confocal microscopy}

Images of fluorescently stained sections were captured using a laser scanning confocal microscopy (LSM880; Carl Zeiss). Tile images of $5 \times 5$ and $10 \times 10$ for the visual cortex and the striatum were acquired, respectively. Each tile of $10 \mu \mathrm{m}$ optical thicknesses was taken using a Plan-Apochromat $20 \times / 0.8$ M27 objective lens (Carl Zeiss), $1.03 \mu$ s pixel time, and four times frame average at $512 \times 512$ resolution. The power of the lasers was $0.4-0.7 \mathrm{~mW}$, which did not induce noticeable fading. 


\subsection{Awake electrophysiological experiments}

Surgery and recordings

Implantation surgery was described in detail earlier (Vandecasteele et al., 2012). Briefly, the animals were anesthetized with isoflurane anesthesia, and one or several craniotomies were performed under stereotaxic guidance. Then, 64-channel silicon probes (NeuroNexus, Ann-Arbor) were implanted in the dorsal striatum (Fig. 4A and C). In two animals, an additional 32-channel silicon probe was implanted in the visual cortex of the same hemisphere. In each of two other animals a bipolar stimulation electrode made of two $50 \mu \mathrm{m}$ thick parylene insulated tungsten wires was also implanted in the visual cortex (AP $4.5 \mathrm{~mm}$ posterior from Bregma; ML $2.8 \mathrm{~mm}$; DV 0.5 and $2 \mathrm{~mm}, 1500 \mu \mathrm{m}$ distance between the tips of the wires, $500 \mu \mathrm{m}$ uninsulated part at each tips). Silicon probes were mounted on custom-made micro-drives to allow their precise vertical movement after implantation. The probes were inserted above the target region, and the micro-drives were attached to the skull with dental cement. The craniotomies were sealed with sterile silicone. Two stainless steel screws were drilled bilaterally over the cerebellum serving as ground and reference for the recordings. Several additional screws were driven into the skull and covered with dental cement to strengthen the implant. Finally, a copper mesh was attached to the skull with dental cement and connected to the ground screw to act as a Faraday cage. After recovery, the probes were moved gradually in $150 \mu \mathrm{m}$ steps per day until the desired position was reached. The $\mathrm{CPu}$ was identified physiologically by the increased number of spiking units. The exact electrode locations were identified post-hoc from histological sections.

\section{Data acquisition}

The operated animals were housed individually in cages and recorded during different navigational tasks. One animal was also recorded during sleep in his home cage. Neuronal recordings were performed daily by connecting the probes to the signal multiplexing headstages attached to a thin and light cable pending from the room ceiling on a trolley system that allowed free movement of the animal (Fig. 10B). The spatial position of the rats during behavioral sessions was monitored using video tracking of two LEDs fixed to the headstage at 30 frames per second, with approx. $3 \mathrm{~mm}$ resolution. The wide-band signal $(0.2-10 \mathrm{kHz})$ acquired at 20K Sample/s using the multiplexer recording system detailed above (KJE-1001, Amplipex Ltd, Hungary). During offline processing, it was low-pass filtered and downsampled to $1250 \mathrm{~Hz}$ for LFP analysis, and high-pass filtered ( $>0.5 \mathrm{kHz}$ ) for spike detection. 


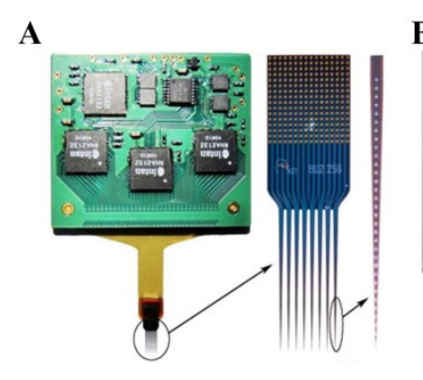

D

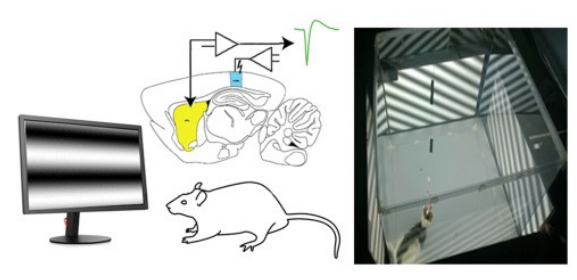

B

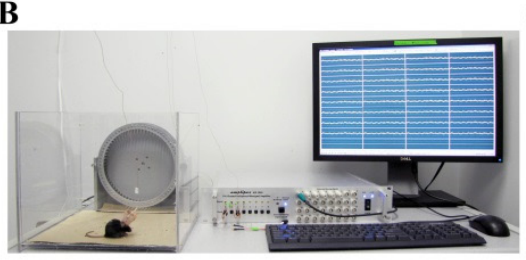

$\mathbf{E}$

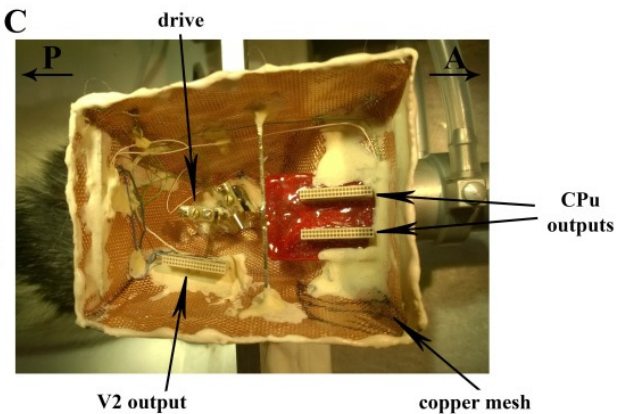

copper mesh

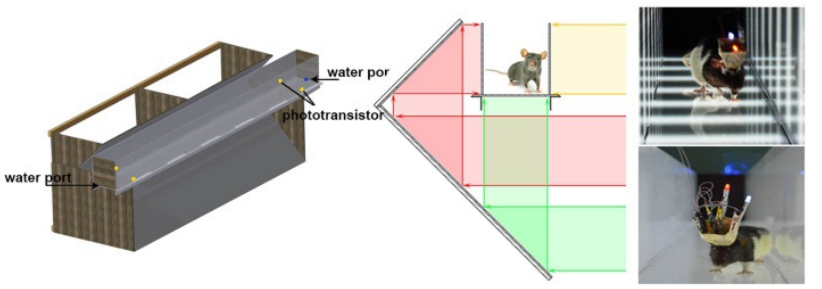

Figure 10. (A) The 256-site silicon probe. Recording sites are arranged vertically at $50-\mu \mathrm{m}$ steps, providing sufficient spatial resolution for unit clustering, yet large vertical coverage $(1550 \mu \mathrm{m})$. (B) Schematic arrangement of the recording setup. (C) The image in surgery about the implanted 64-channel CPu probe and the 32-channel V2 cortical probe. (D) Schematic design of the self-independent visual environment. (E) Schematic design of the self-motion induced visual environmental setup.

Electrical and passive (non-self-motion-induced or self-independent) visual stimulations

A home cage-like visual stimulating environment was created to test the neuronal responses to visual stimuli while the animal was resting. A $60 \times 60 \mathrm{~cm}$ rectangular wooden platform was surrounded by four 24" LED monitors (24M45H-B, LG, Seoul, South Korea) serving as approx. $30 \mathrm{~cm}$ tall walls, and the rats were placed in this stimulation box (Fig. 10D). One stimulus trial consisted of an initial one-second isoluminant gray screen, one second of full-screen stationary grating with sinusoidally modulated luminance in a given orientation ( 8 orientations in $45 \mathrm{deg}$ steps), and with a given spatial density (170, 85, $42.5 \mathrm{~mm} /$ cycle), and finally one second of moving grating, where the grating as mentioned earlier, was sliding orthogonal to its orientation with a given temporal velocity $(1200,600$ and $300 \mathrm{~mm} / \mathrm{s})$. The spatial densities and temporal velocities of the gratings were chosen to match the spectral preference of the caudate neurons reported earlier, when viewed from the center of the cage, and were combined in a way that gave a constant sliding speed of approx. seven cycles/s (Nagy et al., 2010). The frame rate of the monitors was set to $60 \mathrm{~Hz}$; thus, the grating was sled with approx. one-ninth of a cycle in each consecutive frame, which gave the percept of a smooth 
motion. A pseudorandom sequence of visual stimulus trials was generated by random combinations of direction/orientation (eight) and spatial/temporal properties (three), interleaved with trials of blinking full-screen light stimulation at $1 \mathrm{~Hz}$ for three seconds. The final sequence of 2500 three-second long trials was played on all four monitors simultaneously. Each parameter combination was repeated 100 times. The pictures on the monitors were always identical, and the room was otherwise completely dark. The visual stimulation was synchronized with the neuronal data by simultaneously recording a digital code displayed at the top-left corner of the screens by a photodetector. This $1 \times 1 \mathrm{~cm}$ large visual code was not visible to the animals as these parts of the monitors were covered. Animals' behavior was recorded using a web camera, and trials, where they were sleeping instead of random exploration or quiet wakefulness, were excluded. The same stimulation sequence was used for the initial screening of instrastriatal cFos distribution.

In animals with stimulating electrodes in the visual cortex, we also tested single unit and evoked potential responses in the $\mathrm{CPu}$ for electrical stimulation. Bipolar electrical pulses (300 $\mu$ s long $500 \mu \mathrm{A}$ pulses repeated every $300 \mathrm{~ms}$ for five minutes during each session) were delivered, and $\mathrm{CPu}$ recordings were performed while the animals were kept in the same stimulating cage as above with the monitors displaying the uniform gray screens.

\section{Active (self-motion-induced) visual stimulation in the linear maze with a dynamically updatable visual environment}

In order to investigate the responses of $\mathrm{CPu}$ neurons to visual motion induced by the self-motion of the animals, we constructed a linear maze, in which the visual environment could be randomly and instantaneously swapped between uniform grayness and sinusoidally modulated gratings, while the animal was running for water rewards in the maze (Fig. 10E). The visual scene was projected onto the walls and floor of the maze using a video projector (MW663, BenQ, Taipei, Taiwan) in an otherwise dark room. To prevent the animals casting a shadow and blocking the projection on parts of the environment, which is a bottleneck of projecting from above, the maze was constructed of translucent acrylic glass, and the scene was projected on these walls from outside, using a dual mirror system (Fig. 10E). The rats were trained to run back and forth in this maze to collect small water rewards. These droplets were automatically delivered at water-ports located at the two ends of the maze when the animal passed through the infrared gates at the opposite side. These gates were located $10 \mathrm{~cm}$ away 
from the water-ports. After each run, the projected scene was randomly swapped between a uniform gray image and a stationary grating. To match the preferred spatial density of the passive stimulation, fourteen grating cycles were projected along the full length $(200 \mathrm{~cm})$ of the maze. As the rats were running at ca. $85 \mathrm{~cm} / \mathrm{s}$ speed, the temporal velocity of the apparent visual motion ( $\sim 6$ cycles/s) was comparable to that used in the passive stimulation environment.

\section{Post-mortem verification of the electrode locations}

Following the termination of the experiments, animals were deeply anesthetized. All animals were transcardially perfused first with $0.9 \%$ saline solution followed by $4 \%$ paraformaldehyde solution. Brains were sectioned in $60 \mu \mathrm{m}$ thick slices (VT1000S Vibratome, Leica, Wetzlar, Germany) in the coronal plane, and stained using 4',6-Diamidino-2phenylindole dihydrochloride (DAPI) (D8417, Sigma-Aldrich). Locations of the electrode tracks were identified using epifluorescent microscopy (AxioImager, Carl Zeiss, Oberkochen, Germany).

\subsection{Data analysis}

\section{Statistics and data availability}

All data analyses were performed in MATLAB (Mathworks, Natick, MA), unless otherwise noted. For statistical testing, Student's t-test, Wilcoxon rank-sum test, or Kolmogorov-Smirnov tests were used, depending on the distribution of the data, and the sample size (two-tailed). To compensate for the cumulative error due to multiple comparisons, Bonferroni correction was employed. One, two or three asterisks on the figures denote significance levels $<0.05,<0.01$ and $<0.005$, respectively. Modulation indices were always calculated as the ratio of the difference and the sum of two measures.

\section{Image processing}

Separate color channels of the raw micrographic images were exported as 8-bit tiff files. For cFos and PV double immunostaining, cFos-positive, PV-positive, and cFos and PV-double positive neurons were manually counted using the Cell Counter plugin of ImageJ software (NIH, Bethesda, MD). For the estimation of BDA labeled axon density and cFos density, the following automated procedure has been employed: For each slice, a binary mask layer was created in Photoshop (Adobe Systems, San Jose, CA) to void the extra-CPu regions (non-CPu mask) and the apparent artifacts of the BDA channel (intra-CPu mask, e.g., blood vessels). The 
BDA, the cFos, and the mask channels were saved as separate grayscale image files. The cFos image was preprocessed in ImageJ (v1.50). ImageJ (parameters: width: 14 px, minimum distance $7 \mathrm{px}$, threshold 1, detect dark peaks) marked the cFos + somata as red pixels on the grayscale image. All the three image files were loaded in MATLAB, and divided into $512 \times 512$-pixel subregions $(10 \times 10$ pieces $)$. The cFos density was measured at the corresponding image as the number of red (somata) pixels in each subregion, which were not masked in the mask map, divided by the non-masked area. BDA image was thresholded using the mean +4 SD luminosity level of the overall non-masked image. Pixels brighter than this threshold were considered as BDA labeled axons. BDA density was calculated the same way as for the cFos density. Intra-CPu masks were covering not more than $2 \%$ of the total $\mathrm{CPu}$ area at all slices, and were uniformly distributed both along the dorsoventral and mediolateral axes, thus did not affect the measured densities. Data of subregions smaller than 2600 pixels (due to the non-CPu mask) were discarded. The mediolateral and dorsoventral extent of each $\mathrm{CPu}$ slice were normalized when analyzing multiple slices together.

\section{Spike sorting and unit classification}

Neuronal spikes were detected from the digitally high-pass filtered raw signal $(0.5-5 \mathrm{kHz})$ by a threshold crossing-based algorithm (Spikedetekt2; https://github.com/klustateam/spikedetekt2). Detected spikes were automatically sorted using the masked EM algorithm for Gaussians mixtures implemented in KlustaKwik2 (Kadir et al., 2014; https://github.com/klusta-team/klustakwik/), followed by manual adjustment of the clusters using KlustaViewa software (Rossant et al., 2016; https://github.com/klustateam/klustaviewa/) to obtain well-isolated single units. Multiunit or noise clusters were discarded for the analysis. Cluster isolation quality was estimated by calculating the isolation distance and interspike interval index for each cluster (Berényi et al., 2014); poor quality clusters were discarded. Striatal neurons were classified as reported by Schmitzer-Torbert and Redish, 2008 (Schmitzer-Torbert and Redish, 2008), assisted by monosynaptic excitatory and inhibitory interactions between simultaneously recorded, well-isolated units (Oliva et al., 2016). Briefly, five parameters were calculated: Spike-width and spike trough-to-peak time (Berényi et al., 2014), firing rate (FR), the proportion of interspike-intervals exceeding two seconds (ISI2s), and post-spike suppression time (PSST, see Schmitzer-Torbert and Redish, 2008). Neurons with more than $2 \%$ ISI2s, broad spikes, and low FR and PSST were classified 
as TFNs, those with less than $2 \%$ ISI2s, narrow spikes, high FR and low PSST were considered as FFNs, and finally, those with less than $2 \%$ ISI2s, low FR and high PSST were classified as TFNs. Spike waveforms were extracted $([-1-+1] \mathrm{ms}$ around the threshold crossings) from the raw, unfiltered recordings, and their linear trends were removed.

\section{Cross-correlation analysis of single units}

Cross-correlation (CCG) analysis has been applied to detect putative monosynaptic connections (Fujisawa et al., 2008). CCG was calculated as the time-resolved distribution of spike transmission probability between a reference spike train and a temporally shifting target spike train. Co-occurrence counts were normalized by the firing rates of both neurons to get a joint intensity value. A window interval of 2-5 ms with a $1 \mathrm{~ms}$ bin size was used for detecting sharp peaks or troughs, as identifiers of putative monosynaptic connections. Electrical synapses were also detected as 0-1 ms lagged peaks between spike trains of neurons recorded on separate shanks, and less sharp peaks between 6-20 ms were considered as polysynaptic connections. Cell pairs with significant correlations were identified using a jittering method (Fujisawa et al., 2008). For each cell pair, surrogate data sets were constructed by randomly and independently jittering each spike's timestamp with a uniformly distributed value in the range of $[-5,5] \mathrm{ms}$, 1000 times. $99 \%$ acceptance bands were calculated from the surrogate data set CCGs for each bin; multiple comparison error was corrected by introducing 'global significance bands' (Fujisawa et al., 2008). The reference cell of a pair was considered to have an excitatory or inhibitory connection with the referred neuron if any of its CCG bins reached above or below these global bands within the considered time window. Physiologically meaningless detections were not discarded to judge the level of estimation error

\section{Effect of the passive visual stimulation on the FRs}

Peristimulus time histograms (PSTHs) were constructed from the action potential timeseries, triggered by the visual stimulation sequence. Firing rates and modulation indices were calculated for the periods during uniformly grey, stationary grating and moving grating stimulation, and their combinations, respectively. 


\section{Spiking activity in the linear maze}

Coordinates of the detected LEDs were linearized by projecting them onto the axis of the linear maze, smoothed using a 10 frames wide Gaussian kernel, and rounded to $1 \%$ precision. Running trials were considered as periods in the recordings when the animals were traversing from 10 to $90 \%$ of the maze or vice versa. Automated trial extraction was performed by detecting $50 \%$ crossings in the time series of the linearized positions, and each of the crossing detections was tracked backward and forward in time until the velocity dropped below $5 \mathrm{~cm} / \mathrm{s}$. Trials where either of these automatically detected start and stop positions were between 10 and $90 \%$ (i.e. the rat stopped in the middle of the maze) were discarded. Running directions were arbitrarily named 'forward' and 'backward' runs. Spatially resolved firing rate histograms and raster plots were compensated by the occupancy frequency of each bin (Fujisawa et al., 2008). Modulation indices were calculated to express the effect of stripedness on the neuronal firing rates by comparing the FRs during 'striped' and 'blank' trials.

\section{Spatial location related activity in the linear maze}

Rate map, spatial information, selectivity, and sparsity (Oliva et al., 2016) were calculated for each direction separately. A Gaussian kernel $(\mathrm{SD}=5 \mathrm{~cm})$ was applied to both raw maps of spike and occupancy, and a smoothed rate map was constructed by dividing the smoothed spike map by the smoothed occupancy map. A place field was defined as a continuous region, of at least $15 \mathrm{~cm}$, where the firing rate was above $10 \%$ of the peak rate in the maze, the peak firing rate was $>2 \mathrm{~Hz}$ and the spatial coherence was $>0.7$. Place fields with fewer than 50 spikes, those that included the turning position of the track and truncated or overlapping fields were discarded. Place fields in both running directions in the linear track were treated independently. The stability of place fields across conditions was tested by calculating the spatial correlation of the binned mean firing patterns in the striped and uniform trials.

\section{LFP spectral analysis and spike - LFP coupling}

Power spectrum and time-resolved spectral analysis were performed on the low-pass filtered and downsampled (1250 S/s) LFP signals using multitaper spectral analysis. Spectra were whitened by multiplying with the corresponding frequencies, and expressed as decibels (dB). For all local spike-LFP coupling analysis, the median of the local LFP signals recorded on the same shank has been used. For the phase coupling of the CPU neurons to V2 LFP, the median LFP of all V2 recording sites was used instead. Subjectively noisy LFP channels due 
to recording sites with too high impedance were discarded. LFP signals were filtered in five frequency bands (delta: [1-4 Hz], theta: [4-12 Hz], beta: [12-30 Hz], low-gamma: [30-45 Hz], mid-gamma: [45-80 Hz]) with an eighth-order zero phase-lag Butterworth filter, and phase values were obtained using Hilbert-transform. No $50 \mathrm{~Hz}$ notch filtering was used in the data acquisition or during the data analysis. LFP-phase histograms were constructed by taking the corresponding instantaneous LFP phase values corresponding to the isolated single unit spikes, and their deviation from the circular uniform distribution was tested using the Rayleigh-test. The bin-size of the histograms was $18^{\circ}$, and was smoothed by calculating a 5-bin wide moving average of the histograms. The phase values corresponding to the peaks and troughs of the smoothed distributions were taken as preferred and unpreferred phases, respectively. Phase modulation indices (MI) were calculated from smoothed occurrence frequencies of spikes at the preferred and unpreferred phases, to account for possible asymmetric distributions:

$$
M I=\frac{F R_{\max }-F R_{\text {min }}}{F R_{\max }+F R_{\min }}
$$

\section{Cross-region LFP activity analysis}

Median LFP signals were calculated for each recording shank (eight recording sites per shank, eight $\mathrm{CPu}$ shanks, and four cortical shanks). Granger causality was estimated for each $\mathrm{V} 2$ and $\mathrm{CPu}$ signal pairs. Time and frequency-resolved cross-correlation were calculated for each shank pairs (32 in total) the following way: Both signals were iteratively filtered between one and $100 \mathrm{~Hz}$ in $1 \mathrm{~Hz}$ steps, with an eighth order, zero phase-lag passband filters, and their cross-correlations were calculated for $[-100,+100] \mathrm{ms}$ lags, in $2 \mathrm{~ms}$ steps. The magnitudes of the cross-correlograms were taken as the absolute values of its Hilbert transforms for each frequency. To remove the additive distortion caused by the symmetric correlation of the synchronous events (e.g. common noise), the minima of each symmetric time-bin pairs have been subtracted from the same bins. 


\section{SUMMARY}

In my doctoral thesis, my main goal was to summarize the experiments that I performed to investigate the visual responsivity of the $\mathrm{CPu}$ neurons, in order to get a better understanding of the role of $\mathrm{CPu}$ in the complex process of vision. Existing studies focus mainly on skeletomotor and oculomotor functions (i.e., saccades), so the available information about its role in visual and other sensory information processing is very limited. Due to their technical limitations, most studies employed anesthetized or restrained animal models in their experiments. Thus, my primary goal was to establish more naturalistic circumstances during the observation of the striatal activity, yet, keep control of most environmental and behavioral aspects of our experiments. I set out to investigate the striatal neurons and their networks in freely moving rodents, while precisely control their visual experience.

To establish the technical background of these experiments, we had to advance the current state of the art of technology at two points. To minimize the restraint on the behavior of the animals while recording a large number of neurons simultaneously, which latter is a prerequisite of network analysis, we had to reduce the constraints introduced by the headmounted recording system and interconnects. We implemented this by building an innovative novel biosignal amplifier and data acquisition system using time-sharing multiplexing. This technology compresses the signal in a 1:32 ratio, and transmits the neuronal signals in order of magnitude less wires than the traditional neuronal amplifier systems. We validated this new system by comparing the quality of its recordings to commercially available systems as 'industrial standard' and demonstrated that even 512-channel recordings are feasible in rats in a chronic, long-term experiment.

To construct a behavioral testing apparatus where the visual environment can be instantaneously and dynamically altered while the animal can perform realistic exploration (in contrast to the commonly used virtual environments), I designed a maze, where patterns on the transparent walls and floor are projected from the backsides using mirror systems. This way, we could achieve the generation of almost perfect scenes without any blind spots and shadows caused by the animals themselves by blocking the projections with their bodies. 
Using retrograde and anterograde tract-tracing techniques, I identified V1 and V2 as brain areas that may be candidates to send visual information to the striatum and confirmed the dorsomedial part of the caudal striatum as the intrastriatal recipient zone of their efferent fibers. By detecting the product of immediate early gene $\mathrm{cFos}$, we revealed that neurons of this region could indeed be excited through projecting moving visual patterns in the animals' visual fields (i.e., through the passive visual stimulation of the animals), although this effect was only present if the animals were non-anesthetized. It is worth underscoring that visual responsivity of the visual thalamus and primary visual cortex is still preserved during light anesthesia, but according to our results, the striatal processing becomes diminished.

To examine the fine-scale details of the neuronal identities, connectivity, and response profiles in the dorsomedial striatum, I also perform extracellular electrophysiological recordings in experimental environments with various behavior and visual stimulation tasks. Three main classes of neurons (i.e., fast-, phasically-, and tonically firing) were isolated based on their electrophysiological features. Surprisingly, only 25 of the tested 289 neurons were responsive to the passive, self-motion independent visual stimulation. These $\mathrm{pVis}+$ neurons either increased their firing in the appearance of stationary gratings or became inhibited by the movement of the gratings. The majority of them were fast-firing neurons. In this work, I also discuss the obstacles of matching the electrophysiological classification of these neurons to the known histochemical classes.

In the linear maze, however, where the self-motion of the animals induced the visual motion of the wall-projected stationary stripes, the same perceived visual stimulation could entrain an additional $\sim 40 \%$ of all recorded neurons (aVis + cells). These neurons showed a significant change in their firing rate while running in the 'striped' maze compared to the trials with isoluminant uniformly grey walls. Since all pVis + neurons were responsive in this task, from this point, all $\mathrm{pVis}+$ neurons were considered as a subgroup of $\mathrm{aVis}+$ neurons. The same response profile was experienced in the linear maze as during the passive visual stimulation: responsive neurons decreased their firing rate while running in the striped maze.

Lastly, I investigated the functional role of the V2-CPu pathway. The majority ( $80 \%$ ) of the pVis + neurons could also be excited by the electrical stimulation of V2. Moreover, the neurons responsive to this electrical proxy-entrainment were restricted to the same dorsomedial part of the striatum. The time and frequency-resolved cross-coherence estimation of the V2 and 
CPU LFPs revealed that both structures express transient oscillations in the mid gamma band that appear coherently in both $\mathrm{V} 2$ and the $\mathrm{CPu}$ with a constant time lag. This time-resolved gamma coherence became significantly weaker (i.e., the activities of the two structures became less coupled) when the animals perceived self-induced visual motion of the stripes. I also observed, that the visually active $\mathrm{CPu}$ neurons were strongly coupled to these intrastriatal gamma oscillations, but were far less influenced by the theta oscillations, the other prominent rhythm of the striatum.

As the visually active striatal neurons were commonly expressing spatial coding, reward-related activity and other sensory modalities (as reported earlier), I speculate that this gamma synchrony is establishing an effective integration of these multiple information modalities in the $\mathrm{CPu}$, by shaping a proper temporal framework of excitable time-windows to receive synchronized spiking inputs from multiple origins.

My findings emphasize on the integrative and holistic nature of the higher-order sensory processing. Taken, that behavioral adaptation of any organism is established by the continuous adjustment of the effectors based on the percept of the sensors through a gradual sensory-motor translation of inputs to outputs; this is not surprising. Conclusively, the investigation of these processes shall be done in the most possible naturalistic circumstances, rather than using oversimplified reductionist approaches.

\section{ACKNOWLEDGEMENTS}

I wish to express my sincere gratitude to Professors György Benedek, Gyula Sáry, Gábor Jancsó and Dr. Attila Nagy for providing me the opportunity to work in the Department of Physiology and the doctoral program. I acknowledge with thanks the kind of patronage, loving inspiration, and timely guidance, which I have received from my supervisor Dr. Antal Berényi. I would also like to thank some of the people who gave a hand in the creation of this work: Dr. Yuichi Takeuchi and Mari Takeuchi, their help in histology was indispensable while I was expecting my first daughter. This is the kind of support that people never forget. I also wish to express my gratitude to Anikó Jász and Tamás Gyurkovics and other staff members of the Physiology Department who rendered their help during the period of my project work, particularly Gabriella Dósai, whose assistance was essential during my early lab work. 


\section{REFERENCES}

Akhlaghpour, H. et al. (2016) 'Dissociated sequential activity and stimulus encoding in the dorsomedial striatum during spatial working memory’, eLife, 5. doi: 10.7554/eLife.19507.

Alivisatos, A. P. et al. (2012) 'The brain activity map project and the challenge of functional connectomics', Neuron. 2012/06/26, 74(6), pp. 970-974. doi: 10.1016/j.neuron.2012.06.006.

Andermann, M. L. et al. (2011) 'Functional specialization of mouse higher visual cortical areas', Neuron, 72(6), pp. 1025-1039. doi: 10.1016/j.neuron.2011.11.013.

Aosaki, T. et al. (1994) 'Responses of tonically active neurons in the primate's striatum undergo systematic changes during behavioral sensorimotor conditioning.', The Journal of neuroscience : the official journal of the Society for Neuroscience, 14(6), pp. 3969-84. Available at: http://www.ncbi.nlm.nih.gov/pubmed/8207500.

Aosaki, T. et al. (2010) 'Acetylcholine-dopamine balance hypothesis in the striatum: An update', Geriatrics and Gerontology International, 10(SUPPL. 1). doi: 10.1111/j.14470594.2010.00588.x.

Apicella, P. et al. (1991) 'Neglect of contralateral visual stimuli in monkeys with unilateral striatal dopamine depletion.', Behavioural brain research, 46(2), pp. 187-95. Available at: http://www.ncbi.nlm.nih.gov/pubmed/1786125.

Apicella, P. et al. (1998) 'Influence of predictive information on responses of tonically active neurons in the monkey striatum.', Journal of neurophysiology, 80(6), pp. 3341-4. Available at: http://www.ncbi.nlm.nih.gov/pubmed/9862929.

Barnes, T. D. et al. (2005) 'Activity of striatal neurons reflects dynamic encoding and recoding of procedural memories', Nature. 2005/10/21, 437(7062), pp. 1158-1161. doi: 10.1038/nature04053.

Bartos, M., Vida, I. and Jonas, P. (2007) 'Synaptic mechanisms of synchronized gamma oscillations in inhibitory interneuron networks.', Nature reviews. Neuroscience, 8(1), pp. 4556. doi: 10.1038/nrn2044.

Berényi, A. et al. (2014) 'Large-scale, high-density (up to 512 channels) recording of local circuits in behaving animals.', Journal of neurophysiology, 111(5), pp. 1132-49. doi: 10.1152/jn.00785.2013.

Berke, J. (2008) 'Uncoordinated firing rate changes of striatal fast-spiking interneurons during behavioral task performance.', The Journal of neuroscience : the official journal of the Society for Neuroscience, 28(40), pp. 10075-80. doi: 10.1523/JNEUROSCI.2192-08.2008.

Berke, J. D. (2011) 'Functional properties of striatal fast-spiking interneurons.', Frontiers in systems neuroscience, 5(June), p. 45. doi: 10.3389/fnsys.2011.00045. 
Berretta, S., Parthasarathy, H. B. and Graybiel, a M. (1997) 'Local release of GABAergic inhibition in the motor cortex induces immediate-early gene expression in indirect pathway neurons of the striatum.', The Journal of neuroscience : the official journal of the Society for Neuroscience, 17(12), pp. 4752-4763.

Blanche, T. J. et al. (2005) 'Polytrodes: high-density silicon electrode arrays for large-scale multiunit recording', $J$ Neurophysiol. 2004/11/19, 93(5), pp. 2987-3000. doi:

10.1152/jn.01023.2004.

Bonsi, P. et al. (2007) 'Endogenous Serotonin Excites Striatal Cholinergic Interneurons via the Activation of 5-HT 2C, 5-HT6, and 5-HT7 Serotonin Receptors: Implications for Extrapyramidal Side Effects of Serotonin Reuptake Inhibitors', Neuropsychopharmacology, 32(8), pp. 1840-1854. doi: 10.1038/sj.npp.1301294.

Bonsi, P. et al. (2011) 'Centrality of striatal cholinergic transmission in Basal Ganglia function.', Frontiers in neuroanatomy, 5(February), p. 6. doi: 10.3389/fnana.2011.00006.

Boyden, E. S. et al. (2005) 'Millisecond-timescale, genetically targeted optical control of neural activity', Nat Neurosci. 2005/08/24, 8(9), pp. 1263-1268. doi: 10.1038/nn1525.

Buzsaki, G. (2004a) 'Large-scale recording of neuronal ensembles', Nat Neurosci. 2004/04/29, 7(5), pp. 446-451. doi: 10.1038/nn1233.

Buzsaki, G. (2004b) 'Neuronal Oscillations in Cortical Networks', Science, 304(5679), pp. 1926-1929. doi: 10.1126/science.1099745.

Buzsaki, G., Anastassiou, C. A. and Koch, C. (2012) 'The origin of extracellular fields and currents--EEG, ECoG, LFP and spikes’, Nat Rev Neurosci. 2012/05/19, 13(6), pp. 407-420. doi: $10.1038 / \mathrm{nrn} 3241$.

Chuhma, N. et al. (2011) 'Functional connectome of the striatal medium spiny neuron.', The Journal of neuroscience: the official journal of the Society for Neuroscience, 31(4), pp. 1183-92. doi: 10.1523/JNEUROSCI.3833-10.2011.

Clifford, C. W. G. and Ibbotson, M. R. (2002) 'Fundamental mechanisms of visual motion detection: Models, cells and functions', Progress in Neurobiology, 68(6), pp. 409-437. doi: 10.1016/S0301-0082(02)00154-5.

Coizet, V., Overton, P. G. and Redgrave, P. (2007) 'Collateralization of the Tectonigral Projection with Other Major Output Pathways of Superior Colliculus in the Rat', J Comp Neurol, 500, pp. 1034-1049. doi: 10.1002/cne.

Crapse, T. B. and Sommer, M. A. (2008) 'Corollary discharge across the animal kingdom', Nature Reviews Neuroscience, 9(8), pp. 587-600. doi: 10.1038/nrn2457.

Cuturi, L. F. and Macneilage, P. R. (2014) 'Optic flow induces nonvisual self-motion aftereffects', Current Biology. Elsevier Ltd, 24(23), pp. 2817-2821. doi:

10.1016/j.cub.2014.10.015. 
DeCoteau, W. E. et al. (2007) 'Learning-related coordination of striatal and hippocampal theta rhythms during acquisition of a procedural maze task.', Proceedings of the National Academy of Sciences of the United States of America, 104(13), pp. 5644-5649. doi: 10.1073/pnas.0700818104.

Delfs, J. M. et al. (1998) 'Origin of noradrenergic afferents to the shell subregion of the nucleus accumbens: anterograde and retrograde tract-tracing studies in the rat', Brain Research, 806(2), pp. 127-140. doi: 10.1016/S0006-8993(98)00672-6.

Devan, B. D. and White, N. M. (1999) 'Parallel Information Processing in the Dorsal Striatum : Relation to Hippocampal Function’, 19(7), pp. 2789-2798.

Du, J. et al. (2009) 'High-resolution three-dimensional extracellular recording of neuronal activity with microfabricated electrode arrays', J Neurophysiol. 2008/12/19, 101(3), pp. 1671-1678. doi: 10.1152/jn.90992.2008.

$\mathrm{Du}$, J. et al. (2011) 'Multiplexed, high density electrophysiology with nanofabricated neural probes', PLoS One. 2011/10/25, 6(10), p. e26204. doi: 10.1371/journal.pone.0026204.

Fries, P., Nikolić, D. and Singer, W. (2007) 'The gamma cycle.', Trends in neurosciences, 30(7), pp. 309-16. doi: 10.1016/j.tins.2007.05.005.

Fujisawa, S. et al. (2008) 'Behavior-dependent short-term assembly dynamics in the medial prefrontal cortex.', Nature neuroscience, 11(7), pp. 823-33. doi: 10.1038/nn.2134.

Geisler, C. et al. (2007) 'Hippocampal place cell assemblies are speed-controlled oscillators.', Proceedings of the National Academy of Sciences of the United States of America, 104(19), pp. 8149-54. doi: 10.1073/pnas.0610121104.

Gerfen, C. et al. (1990) 'D1 and D2 dopamine receptor-regulated gene expression of striatonigral and striatopallidal neurons', Science, 250(4986), pp. 1429-1432. doi: 10.1126/science. 2147780 .

Gizzi, M. S. et al. (1990) 'Selectivity for orientation and direction of motion of single neurons in cat striate and extrastriate visual cortex.', Journal of neurophysiology, 63(6), pp. 15291543.

Gombkötö, P. et al. (2011) 'Neuronal code of spatial visual information in the caudate nucleus.', Neuroscience, 182, pp. 225-31. doi: 10.1016/j.neuroscience.2011.02.048.

Goodale, M. A. and Milner, A. D. (1992) 'Separate visual pathways for perception and action', Trends in Neurosciences, 15(1), pp. 20-25. doi: 10.1016/0166-2236(92)90344-8.

Graybiel, A. M. (1990) 'Neurotransmitters and neuromodulators in the basal ganglia.', Trends in neurosciences, 13(7), pp. 244-54. Available at:

http://www.ncbi.nlm.nih.gov/pubmed/1695398.

Graziano, M. A. and Gross, C. (1993) 'A bimodal map of space: somatosensory receptive fields in the macaque putamen with corresponding visual receptive fields', Experimental Brain Research, 97(1). doi: 10.1007/BF00228820. 
Harrison, R. R. (2008) 'The design of integrated circuits to observe brain activity', Proceedings of the IEEE, 96(7), pp. 1203-1216. Available at:

http://www.ece.utah.edu/ harrison/ProcIEEE2008.pdf.

Hernandez, L. F. et al. (2013) 'Selective effects of dopamine depletion and L-DOPA therapy on learning-related firing dynamics of striatal neurons.', The Journal of neuroscience : the official journal of the Society for Neuroscience, 33(11), pp. 4782-95. doi: 10.1523/JNEUROSCI.3746-12.2013.

Hitier, M., Besnard, S. and Smith, P. F. (2014) 'Vestibular pathways involved in cognition.', Frontiers in integrative neuroscience, 8(July), p. 59. doi: 10.3389/fnint.2014.00059.

Hjorth, J., Blackwell, K. T. and Kotaleski, J. H. (2009) 'Gap junctions between striatal fastspiking interneurons regulate spiking activity and synchronization as a function of cortical activity.', The Journal of Neuroscience, 29(16), pp. 5276-86. doi:

10.1523/JNEUROSCI.6031-08.2009.

Horst, N. K. and Laubach, M. (2013) 'Reward-related activity in the medial prefrontal cortex is driven by consumption', Frontiers in Neuroscience, 7(7 APR), pp. 1-15. doi:

10.3389/fnins.2013.00056.

Horvitz, J. C. (2009) 'Stimulus-response and response-outcome learning mechanisms in the striatum', Behavioural Brain Research, 199(1), pp. 129-140. doi: 10.1016/j.bbr.2008.12.014.

Hughes, P. and Dragunow, M. (1995) 'Induction of immediate-early genes and the control of neurotransmitter-regulated gene expression within the nervous system', Pharmacological Rewiews.

Hunnicutt, B. J. et al. (2016) 'A comprehensive excitatory input map of the striatum reveals novel functional organization', eLife, 5, p. e19103. doi: 10.7554/eLife.19103.

Ibanez-Sandoval, O. et al. (2011) 'A Novel Functionally Distinct Subtype of Striatal Neuropeptide Y Interneuron', Journal of Neuroscience, 31(46), pp. 16757-16769. doi: 10.1523/JNEUROSCI.2628-11.2011.

Jones, M. W. and Wilson, M. A. (2005) 'Phase precession of medial prefrontal cortical activity relative to the hippocampal theta rhythm.', Hippocampus, 15(7), pp. 867-73. doi: 10.1002/hipo.20119.

Kadir, S. N., Goodman, D. F. M. and Harris, K. D. (2014) 'High-Dimensional Cluster Analysis with the Masked EM Algorithm', Neural Computation, 26(11), pp. 2379-2394. doi: 10.1162/NECO_a_00661.

Kamishina, H. et al. (2008) 'Striatal projections from the rat lateral posterior thalamic nucleus', Brain Research, 1204, pp. 24-39. doi: 10.1016/j.brainres.2008.01.094.

Kawaguchi, Y. (1993) 'Physiological, morphological, and histochemical characterization of three classes of interneurons in rat neostriatum.', The Journal of neuroscience : the official journal of the Society for Neuroscience, 13(11), pp. 4908-4923. doi: 10.1016/S09218696(05)81133-8. 
Khibnik, L. A., Tritsch, N. X. and Sabatini, B. L. (2014) 'A direct projection from mouse primary visual cortex to dorsomedial striatum', PloS one, 9(8), p. e104501. doi: 10.1371/journal.pone.0104501.

Klausberger, T. et al. (2003) 'Brain-state- and cell-type-specific firing of hippocampal interneurons in vivo.', Nature, 421(6925), pp. 844-8. doi: 10.1038/nature01374.

Kondabolu, K. et al. (2016) 'Striatal cholinergic interneurons generate beta and gamma oscillations in the corticostriatal circuit and produce motor deficits', Proceedings of the National Academy of Sciences, p. 201605658. doi: 10.1073/pnas.1605658113.

Koós, T. and Tepper, J. M. (1999) 'Inhibitory control of neostriatal projection neurons by GABAergic interneurons.', Nature neuroscience, 2(5), pp. 467-72. doi: 10.1038/8138.

Koos, T., Tepper, J. M. and Wilson, C. J. (2004) 'Comparison of IPSCs evoked by spiny and fast-spiking neurons in the neostriatum', J Neurosci, 24(36), pp. 7916-7922. doi:

10.1523/JNEUROSCI.2163-04.2004\r24/36/7916 [pii].

Kravitz, A. V, Tye, L. D. and Kreitzer, A. C. (2012) 'Distinct roles for direct and indirect pathway striatal neurons in reinforcement', Nature Neuroscience, 15(6), pp. 816-818. doi: 10.1038/nn.3100.

Lein, E. S. et al. (2007) 'Genome-wide atlas of gene expression in the adult mouse brain', Nature. 2006/12/08, 445(7124), pp. 168-176. doi: 10.1038/nature05453.

Licheri, V. et al. (2018) 'Complex Control of Striatal Neurotransmission by Nicotinic Acetylcholine Receptors via Excitatory Inputs onto Medium Spiny Neurons', The Journal of Neuroscience, 38(29), pp. 6597-6607. doi: 10.1523/JNEUROSCI.0071-18.2018.

Lichtman, J. W. and Denk, W. (2011) 'The big and the small: challenges of imaging the brain's circuits', Science. 2011/11/05, 334(6056), pp. 618-623. doi:

10.1126/science. 1209168 .

Logothetis, N. K. (2003) 'The underpinnings of the BOLD functional magnetic resonance imaging signal', J Neurosci. 2003/05/24, 23(10), pp. 3963-3971. Available at:

http://www.ncbi.nlm.nih.gov/pubmed/12764080.

Luo, R. et al. (2013) 'Direct and GABA-mediated indirect effects of nicotinic ACh receptor agonists on striatal neurones.', The Journal of physiology, 591(Pt 1), pp. 203-17. doi:

10.1113/jphysiol.2012.241786.

Madisen, L. et al. (2012) 'A toolbox of Cre-dependent optogenetic transgenic mice for lightinduced activation and silencing.', Nature neuroscience. Nature Publishing Group, 15(5), pp. 793-802. doi: 10.1038/nn.3078.

Mallet, N. et al. (2005) 'Feedforward inhibition of projection neurons by fast-spiking GABA interneurons in the rat striatum in vivo.', The Journal of neuroscience: the official journal of the Society for Neuroscience, 25(15), pp. 3857-69. doi: 10.1523/JNEUROSCI.5027-04.2005. 
McGinley, M. J. et al. (2015) 'Waking State: Rapid Variations Modulate Neural and Behavioral Responses', Neuron. Elsevier Inc., 87(6), pp. 1143-1161. doi:

10.1016/j.neuron.2015.09.012.

van der Meer, M. A. A. et al. (2010) 'Integrating early results on ventral striatal gamma oscillations in the rat', Frontiers in Neuroscience, 4(SEP), pp. 1-12. doi:

10.3389/fnins.2010.00300.

van der Meer, M. A. A. and Redish, A. D. (2009) 'Low and High Gamma Oscillations in Rat Ventral Striatum have Distinct Relationships to Behavior, Reward, and Spiking Activity on a Learned Spatial Decision Task.', Frontiers in integrative neuroscience, 3, p. 9. doi:

10.3389/neuro.07.009.2009.

van der Meer, M. A. A. and Redish, A. D. (2011) 'Theta Phase Precession in Rat Ventral Striatum Links Place and Reward Information', The Journal of Neuroscience, 31(8), pp. 2843-2854. doi: 10.1523/JNEUROSCI.4869-10.2011.

Montero, V. M. and Jian, S. (1995) 'Induction of c-los protein by patterned visual stimulation in central visual pathways of the rat', 690, pp. 189-199.

Nagy, A. et al. (2010) 'Spectral receptive field properties of visually active neurons in the caudate nucleus', Neuroscience Letters, 480(2), pp. 148-153. doi:

10.1016/j.neulet.2010.06.030.

Nagy, A. J. et al. (2011) 'Direct projection from the visual associative cortex to the caudate nucleus in the feline brain', Neuroscience Letters, 503(1), pp. 52-57. doi:

10.1016/j.neulet.2011.08.007.

Nagypál, T. et al. (2015) 'Activity of caudate nucleus neurons in a visual fixation paradigm in behaving cats', PLOS ONE, 10(11), pp. 1-18. doi: 10.1371/journal.pone.0142526.

Nasr, S. and Rosas, H. D. (2016) 'Impact of Visual Corticostriatal Loop Disruption on Neural Processing within the Parahippocampal Place Area', The Journal of Neuroscience, 36(40), pp. 10456-10471. doi: 10.1523/JNEUROSCI.0741-16.2016.

Nelson, A. B. et al. (2014) 'Striatal cholinergic interneurons drive GABA release from dopamine terminals', Neuron. Elsevier Inc., 82(1), pp. 63-70. doi:

10.1016/j.neuron.2014.01.023.

Nicolelis, M. A. et al. (1997) 'Reconstructing the engram: simultaneous, multisite, many single neuron recordings', Neuron. 1997/04/01, 18(4), pp. 529-537. Available at: http://www.ncbi.nlm.nih.gov/pubmed/9136763.

O'Keefe, J. and Dostrovsky, J. (1971) 'The hippocampus as a spatial map. Preliminary evidence from unit activity in the freely-moving rat.', Brain research, 34(1), pp. 171-5.

Oliva, A. et al. (2016) 'Spatial coding and physiological properties of hippocampal neurons in the Cornu Ammonis subregions.', Hippocampus, 26(12), pp. 1593-1607. doi: 10.1002/hipo.22659. 
Olsson 3rd, R. H. et al. (2005) 'Band-tunable and multiplexed integrated circuits for simultaneous recording and stimulation with microelectrode arrays', IEEE Trans Biomed Eng. 2005/07/27, 52(7), pp. 1303-1311. doi: 10.1109/TBME.2005.847540.

Oorschot, D. E. (2010) 'Cell Types in the Different Nuclei of the Basal Ganglia', in Steiner, H. and Tseng, K. (eds) Handbook of Basal Ganglia Structure and Function. London: Academic Press, pp. 63-74. doi: 10.1016/B978-0-12-374767-9.00003-2.

Paxinos, G. and Watson, C. (2013) The Rat Brain in Stereotaxic Coordinates. 7th ed. London: Academic Press.

Pennartz, C. M. A. et al. (2011) 'The hippocampal-striatal axis in learning, prediction and goal-directed behavior.', Trends in neurosciences, 34(10), pp. 548-59. doi: 10.1016/j.tins.2011.08.001.

Petruno, S. K., Clark, R. E. and Reinagel, P. (2013) 'Evidence That Primary Visual Cortex Is Required for Image, Orientation, and Motion Discrimination by Rats', PLoS ONE, 8(2), pp. 1-10. doi: 10.1371/journal.pone.0056543.

Prakash, R. et al. (2012) 'Two-photon optogenetic toolbox for fast inhibition, excitation and bistable modulation', Nat Methods. 2012/11/22, 9(12), pp. 1171-1179. doi:

10.1038/nmeth.2215.

Redgrave, P., Prescott, T. J. and Gurney, K. (1999) 'The basal ganglia: a vertebrate solution to the selection problem?', Neuroscience, 89(4), pp. 1009-23.

Reig, R. and Silberberg, G. (2014) 'Multisensory integration in the mouse striatum.', Neuron, 83(5), pp. 1200-12. doi: 10.1016/j.neuron.2014.07.033.

Rokszin, A. et al. (2010) 'Visual pathways serving motion detection in the mammalian brain', Sensors, 10(4), pp. 3218-3242. doi: 10.3390/s100403218.

Rossant, C. et al. (2016) 'Spike sorting for large, dense electrode arrays.', Nature neuroscience, 19(4), pp. 634-41. doi: 10.1038/nn.4268.

Roth, M. M. et al. (2015) 'Thalamic nuclei convey diverse contextual information to layer 1 of visual cortex’, Nature Neuroscience, 19(2), pp. 299-307. doi: 10.1038/nn.4197.

Rubio-Garrido, P. et al. (2009) 'Thalamic input to distal apical dendrites in neocortical layer 1 is massive and highly convergent', Cerebral Cortex, 19(10), pp. 2380-2395. doi: $10.1093 /$ cercor/bhn259.

Russo, G. et al. (2013) 'Dynamics of action potential firing in electrically connected striatal fast-spiking interneurons', Frontiers in Cellular Neuroscience, 7. doi:

10.3389/fncel.2013.00209.

Schmitzer-Torbert, N. C. and Redish, A. D. (2008) 'Task-dependent encoding of space and events by striatal neurons is dependent on neural subtype', Neuroscience, 153(2), pp. 349360. doi: 10.1016/j.neuroscience.2008.01.081. 
Schulz, J. M. et al. (2011) 'Enhanced high-frequency membrane potential fluctuations control spike output in striatal fast-spiking interneurones in vivo.', The Journal of physiology, 589(Pt 17), pp. 4365-81. doi: 10.1113/jphysiol.2011.212944.

Schwindel, C. D. et al. (2014) 'Long-Term Recordings Improve the Detection of Weak Excitatory-Excitatory Connections in Rat Prefrontal Cortex', The Journal of Neuroscience, 34(16), pp. 5454-5467. doi: 10.1523/JNEUROSCI.4350-13.2014.

Sereno, M. I. and Allmann, J. M. (1991) 'Cortical Visual Areas in Mammals', in Leventhal, A. G. (ed.) The Neural Basis of Visual Function. London: Macmillan, pp. 160-172.

Serizawa, M. et al. (1994) 'Corticostriatal and corticotectal projections from visual cortical areas 17, 18 and 18a in the pigmented rat.', Archives of histology and cytology, 57(5), pp. $493-507$.

Sharott, A. et al. (2009) 'Different Subtypes of Striatal Neurons Are Selectively Modulated by Cortical Oscillations', The Journal of Neuroscience, 29(14), pp. 4571-4585. doi: 10.1523/JNEUROSCI.5097-08.2009.

Siapas, A. G., Lubenov, E. V and Wilson, M. A. (2005) 'Prefrontal phase locking to hippocampal theta oscillations.', Neuron, 46(1), pp. 141-51. doi: 10.1016/j.neuron.2005.02.028.

Sommer, M. A. and Wurtz, R. H. (2002) 'A Pathway in Primate Brain for Internal Monitoring of Movements’, Science, 296(5572), pp. 1480-1482. doi: 10.1126/science.1069590.

Sperry, R. W. (1950) 'Neural basis of the spontaneous optokinetic response produced by visual inversion.', Journal of comparative and physiological psychology, 43(6), pp. 482-9. doi: $10.1037 / \mathrm{h} 0055479$.

Strecker, R. E. et al. (1985) 'Caudate unit activity in freely moving cats: effects of phasic auditory and visual stimuli', Brain Research, 329(1-2), pp. 350-353. doi: 10.1016/00068993(85)90548-7.

Szuts, T. A. et al. (2011) 'A wireless multi-channel neural amplifier for freely moving animals', Nat Neurosci. 2011/01/18, 14(2), pp. 263-269. doi: 10.1038/nn.2730.

Takeuchi, Y. et al. (2017) 'Afferent Fiber Remodeling in the Somatosensory Thalamus of Mice as a Neural Basis of Somatotopic Reorganization in the Brain and Ectopic Mechanical Hypersensitivity after Peripheral Sensory Nerve Injury', eneuro, 4(2), p. ENEURO.034516.2017. doi: 10.1523/ENEURO.0345-16.2017.

Taverna, S., Canciani, B. and Pennartz, C. M. A. (2007) 'Membrane properties and synaptic connectivity of fast-spiking interneurons in rat ventral striatum.', Brain research, 1152, pp. 49-56. doi: 10.1016/j.brainres.2007.03.053.

Tepper, J. M. et al. (2010) 'Heterogeneity and diversity of striatal GABAergic interneurons.', Frontiers in neuroanatomy, 4(December), p. 150. doi: 10.3389/fnana.2010.00150. 
Tepper, J. M. et al. (2018) 'Heterogeneity and Diversity of Striatal GABAergic Interneurons: Update 2018', Frontiers in Neuroanatomy, 12(November), pp. 1-14. doi: 10.3389/fnana.2018.00091.

Thorn, C. A. and Graybiel, A. M. (2014) 'Differential Entrainment and Learning-Related Dynamics of Spike and Local Field Potential Activity in the Sensorimotor and Associative Striatum', The Journal of Neuroscience, 34(8), pp. 2845-2859. doi:

10.1523/JNEUROSCI.1782-13.2014.

Tye, K. M. and Deisseroth, K. (2012) 'Optogenetic investigation of neural circuits underlying brain disease in animal models', Nat Rev Neurosci. 2012/03/21, 13(4), pp. 251-266. doi: $10.1038 / \mathrm{nrn} 3171$.

Vandecasteele, M. et al. (2007) 'Electrical synapses in basal ganglia.', Reviews in the neurosciences, 18(1), pp. 15-35. doi: 10.1515/REVNEURO.2007.18.1.15.

Vandecasteele, M. et al. (2012) 'Large-scale recording of neurons by movable silicon probes in behaving rodents.', Journal of visualized experiments : JoVE, (61).

Viventi, J. et al. (2011) 'Flexible, foldable, actively multiplexed, high-density electrode array for mapping brain activity in vivo', Nat Neurosci. 2011/11/15, 14(12), pp. 1599-1605. doi: 10.1038/nn.2973.

Wilson, C. J. (2014) 'The Sensory Striatum', Neuron, 83(5), pp. 999-1001. doi: 10.1016/j.neuron.2014.08.025.

Wilson, C. J. and Kawaguchi, Y. (1996) 'The origins of two-state spontaneous membrane potential fluctuations of neostriatal spiny neurons.', The Journal of neuroscience : the official journal of the Society for Neuroscience, 16(7), pp. 2397-410.

Wilson, M. A. and McNaughton, B. L. (1993) 'Dynamics of the hippocampal ensemble code for space’, Science. 1993/08/20, 261(5124), pp. 1055-1058. Available at:

http://www.ncbi.nlm.nih.gov/pubmed/8351520.

Winkel, J. et al. (2016) 'Focal striatum lesions impair cautiousness in humans', Cortex. Elsevier Ltd, 85, pp. 37-45. doi: 10.1016/j.cortex.2016.09.023.

Yamamoto, S. et al. (2012) 'What and Where Information in the Caudate Tail Guides Saccades to Visual Objects', Journal of Neuroscience, 32(32), pp. 11005-11016. doi: 10.1523/JNEUROSCI.0828-12.2012.

Yanike, M. and Ferrera, V. P. (2014) 'Interpretive monitoring in the caudate nucleus', eLife, 3(November), pp. 1-16. doi: 10.7554/eLife.03727.

Yizhar, O. et al. (2011) 'Optogenetics in neural systems', Neuron. 2011/07/13, 71(1), pp. 934. doi: 10.1016/j.neuron.2011.06.004.

Zhou, F.-M., Wilson, C. J. and Dani, J. A. (2002) 'Cholinergic interneuron characteristics and nicotinic properties in the striatum.', Journal of neurobiology, 53(4), pp. 590-605. doi:

10.1002/neu.10150. 
I. 


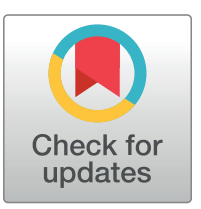

\section{openaccess}

Citation: Nagy AJ, Takeuchi Y, Berényi A (2018) Coding of self-motion-induced and selfindependent visual motion in the rat dorsomedial striatum. PLoS Biol 16(6): e2004712. https://doi. org/10.1371/journal.pbio.2004712

Academic Editor: Carl Petersen, EPFL, Switzerland

Received: November 3, 2017

Accepted: June 11, 2018

Published: June 25, 2018

Copyright: @ 2018 Nagy et al. This is an open access article distributed under the terms of the Creative Commons Attribution License, which permits unrestricted use, distribution, and reproduction in any medium, provided the original author and source are credited.

Data Availability Statement: All relevant data are within the paper and its Supporting Information files.

Funding: Momentum Grant of the Hungarian Academy of Sciences www.mta.hu. Received by $A B$. The funder had no role in study design, data collection and analysis, decision to publish, or preparation of the manuscript. European Research Council erc.europa.eu (grant number EU-FP7-ERC2013-Starting grant (No.337075)). Recieved by AB. The funder had no role in study design, data collection and analysis, decision to publish, or preparation of the manuscript. National Research,
RESEARCH ARTICLE

\section{Coding of self-motion-induced and self- independent visual motion in the rat dorsomedial striatum}

\author{
Anett J. Nagy ${ }^{1}$, Yuichi Takeuchi ${ }^{1}$, Antal Berényi ${ }^{1,2 *}$ \\ 1 MTA-SZTE "Momentum" Oscillatory Neuronal Networks Research Group, Department of Physiology, \\ University of Szeged, Szeged, Hungary, 2 Neuroscience Institute, New York University, New York, New \\ York, United States of America \\ *drberenyi@gmail.com
}

\section{Abstract}

Evolutionary development of vision has provided us with the capacity to detect moving objects. Concordant shifts of visual features suggest movements of the observer, whereas discordant changes are more likely to be indicating independently moving objects, such as predators or prey. Such distinction helps us to focus attention, adapt our behavior, and adjust our motor patterns to meet behavioral challenges. However, the neural basis of distinguishing self-induced and self-independent visual motions is not clarified in unrestrained animals yet. In this study, we investigated the presence and origin of motion-related visual information in the striatum of rats, a hub of action selection and procedural memory. We found that while almost half of the neurons in the dorsomedial striatum are sensitive to visual motion congruent with locomotion (and that many of them also code for spatial location), only a small subset of them are composed of fast-firing interneurons that could also perceive self-independent visual stimuli. These latter cells receive their visual input at least partially from the secondary visual cortex (V2). This differential visual sensitivity may be an important support in adjusting behavior to salient environmental events. It emphasizes the importance of investigating visual motion perception in unrestrained animals.

\section{Author summary}

Evolution of vision has provided us with the capacity to detect moving objects. However, visual motion perception may occur both because of the movement of an observer in an otherwise stationary environment and because of independently moving observed objects. Distinguishing these two sources or simply recognizing truly moving objects when the observer is also moving is essential for survival; it allows the reliable detection of predators or prey in real-world situations. In this study, we investigated the presence and origin of motion-related visual information in the striatum of rats, a hub of action selection and procedural memory. We found that while many of the striatal neurons responded to the apparent visual motion while the rat was running in an environment with striped walls, only a subset of them could also respond to moving stripes with similar properties if 
Development and Innovation Office www.nkfih.hu (grant number EFOP-3.6.1-16-2016-00008). Recieved by AB. The funder had no role in study design, data collection and analysis, decision to publish, or preparation of the manuscript. Uehara Memorial Fund www.taisho-holdings.co.jp. Recieved by YT. The funder had no role in study design, data collection and analysis, decision to publish, or preparation of the manuscript.

Competing interests: I have read the journal's policy and the authors of this manuscript have the following competing interests. $A B$ and AJN are owners of Amplipex Ltd and Neunos LLC., Szeged, Hungary, which manufacture signal-multiplexed headstages and neurostimulator devices.

Abbreviations: a.u., arbitrary unit; AP, anteroposterior; AuCx, auditory cortex; aVis, active visual; $B D A$, biotinylated dextran amine; $B S A$, bovine serum albumin; $\mathrm{CPu}$, caudate putamen; DAPI, 4',6-Diamidino-2-phenylindole dihydrochloride; $\mathrm{dB}$, decibel; DLG, dorsolateral geniculate nucleus; DV, dorsoventral; EPSP, excitatory postsynaptic potential; FFN, fast-firing neuron; FR, firing rate; IEG, immediate early gene; ISI, interspike interval; ISI2s, interspike interval exceeding $2 \mathrm{~s}$; ITCN, Image-based Tool for Counting Nuclei; LCD, liquid crystal display; LFP, local field potential; ML, mediolateral; NGS, normal goat serum; PB, phosphate buffer; PBS, phosphate-buffered saline; PBS-X, PBS containing $0.3 \%$ Triton X-100; PBS-XBSA, PBS-X containing $1 \%$ BSA; PFA, paraformaldehyde; PFN, phasically firing neuron; PSST, postspike suppression time; PV, parvalbumin; $p$ Vis, passive visual; SUA, singleunit activity; TFN, tonically firing neuron; $\mathrm{V} 1$, primary visual cortex; $\mathrm{V} 2$, secondary visual cortex; V2L, lateral secondary visual cortex; V2ML, mediolateral secondary visual cortex; V2MM, mediomedial secondary visual cortex. watched passively. This differential visual sensitivity may be an important mechanism of adjusting behavior to salient environmental events. The need for congruent locomotion to evoke neuronal responses in many of the recorded neurons emphasizes the importance of investigating visual motion perception in unrestrained animals.

\section{Introduction}

Besides the well-known motor functions of the basal ganglia [1], there is massive evidence for its role in higher-order integrative functions. While the ventral part of the caudate putamen $(\mathrm{CPu})$ is mainly associated with reward and motivation-related processes (e.g., reinforcement [2]), the dorsal part is involved in executive functions based on both stimulus response learning [3] and procedural memory [4-6]. Neurons of this dorsal sensorimotor striatum can perceive elementary visual cues representing luminance changes [7,8] and visual motion [9-12] and integrate them with other modalities $[13,14]$ to serve their function in multiple potential ways. On one hand, the self-motion-induced changes in the visual world (i.e., due to the dislocation of the observer) would serve as feedback for both corollary discharge [15-17] and for refining motor outputs [18]. On the other hand, the detection of the self-motion-independent object motion in an otherwise stationary environment would be very important for recognizing approaching predators and other threats or escaping prey. This latter process may be important for survival by facilitating appropriate action selection through perceptual decisionmaking [19].

Previous experiments that studied the motion sensitivity of the $\mathrm{CPu}$ at the single-neuron level employed anesthetized or restrained animals. Thus, they were not designed for deciphering if the $\mathrm{CPu}$ is equally sensitive for the self-motion-induced (i.e., dislocations of the visual scene congruent with self-motion) and the self-motion-independent object motion. More profoundly, there is no existing evidence that the $\mathrm{CPu}$ neurons can distinguish between these two conditions at all nor whether they can integrate the visual motion percepts with spatial representations or other aspects of striatal information processing.

To answer this question, we investigated the response profiles of the dorsal $\mathrm{CPu}$ neurons to self-independent and self-motion-induced visual motion stimuli in freely moving rats. We did this by conducting large-scale, high-density extracellular recordings. We also made efforts to show the role of the corticostriatal pathway in shaping these responses, as there is no consensus regarding the origin of the striatal visual information either [7,20-22].

\section{Results}

We conducted our experiments on male Long-Evans rats ( $N=32$, 285-655 g; 3-12 mo old). The intermediate part of the dorsomedial striatum has been reported to display visual stimulus-induced electrophysiological responsivity in many species [7,9-11]. This region has also been reported to receive axons from both the A18 and A18b secondary visual cortical areas and to be the exclusive striatal recipient of innervation from the primary visual cortex (V1) $[20,23]$. To confirm this finding, we injected FluoroGold to the dorsomedial $\mathrm{CPu}$ ( $N=4$ rats, Fig 1 A) and identified regions with retrogradely labeled neuronal somata. Similarly to previous results obtained in mice and rats [7,23], we found that neurons of the secondary visual cortex (V2, and also the most rostral part of the V1) send axonal projections to the injected area of the $\mathrm{CPu}$ (Fig 1B). Based on their large somata, their thick apical dendrites, and their restricted layer $\mathrm{V}$ locations, the labeled neurons are putative pyramidal neurons. We did not 
A

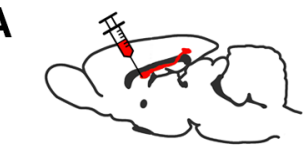

Fluorogold
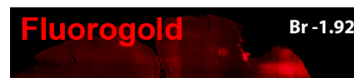

3

CPy

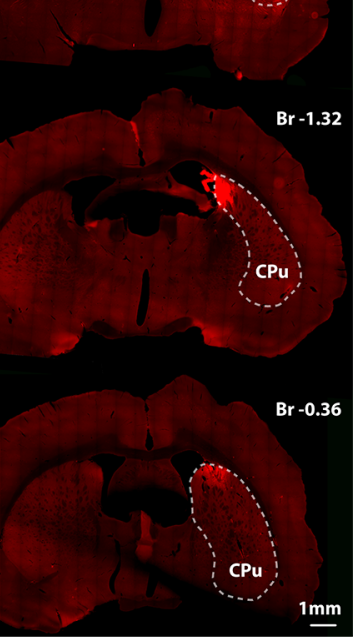

$1 \mathrm{~mm}$
B

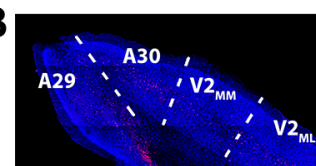

Fluorogold

Niss!

Br -4.68

V1
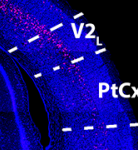

AuC
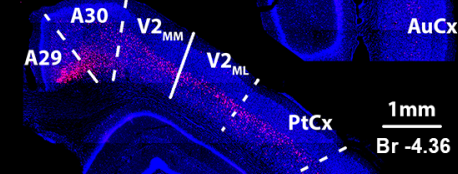

S1

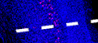

AuCx

$--$

$30 \mu \mathrm{m}$

V2

Fig 1. Anatomical connectivity of the dorsomedial striatum. (A) Injection schematic and representative micrographic images of the striatal injection site of the retrograde tracer Fluorogold. (B) Retrogradely labeled cell bodies were found in the V1 and V2 ipsilaterally, as well as in the posterior ("Po") and LPMR thalamus. Note the lack of labeling at the DLG. Bottom panel shows a magnified image of the area $\mathrm{V} 2_{\mathrm{ML}}$ of the middle panel. The thick apical dendrites and the location of the somata suggest that the labeled neurons are putative pyramidal neurons. AuCx, auditory cortex; $\mathrm{Br}$, bregma; $\mathrm{CPu}$, caudate putamen; DLG, dorsolateral geniculate nucleus; LPMR, lateralposteromedial; V1, primary visual cortex; V2, secondary visual cortex; $\mathrm{V} 2_{\mathrm{L}}$, lateral secondary visual cortex; $\mathrm{V} 2_{\mathrm{ML}}$, mediolateral secondary visual cortex; $\mathrm{V} 2_{\mathrm{MM}}$, mediomedial secondary visual cortex.

https://doi.org/10.1371/journal.pbio.2004712.g001

find any labeled neurons at the more caudal parts of the V1 or in the lateral geniculate nucleus, but the lateral-posteromedial and posterior nuclei of the thalamus were densely labeled.

To evaluate the colocalization of the axons originating from the visual cortex and the visually active $\mathrm{CPu}$ neurons on the fine scale, we injected biotinylated dextran amine (BDA) at the V2 locations identified in our retrograde tracing experiment (Fig 2A). After $1 \mathrm{wk}$ of survival, the animals were placed into a $60 \times 60 \mathrm{~cm}$ large box whose $35 \mathrm{~cm}$ tall walls featured 4 liquid crystal display (LCD) monitors to provide a self-motion-independent, passive visual ( $\mathrm{pVis}$ ) stimulation. For the initial $6 \mathrm{~h}$, the monitors were turned off, and the animals were kept in darkness. Then, a pseudorandom sequence of moving visual gratings and blinking lights was displayed on the monitors for $1 \mathrm{~h}$ while the animals were randomly exploring the box (Fig 2A, for details of the $\mathrm{pVis}$ stimulation, see the Materials and methods section). The control animals were kept in darkness for $7 \mathrm{~h}$ without any stimulation. The rats were overanesthetized and perfused. Coronal sections of their brains were developed to visualize BDA-positive axons and the expression of the cFos (Fig 2B). We also tested if the cFos+ neurons were parvalbumin positive $(\mathrm{PV}+)$. We found that the presence of the two markers were almost mutually exclusive (as demonstrated by the anti-cFos and anti-PV double immunostaining in 4 sections of 1 rat, 
A

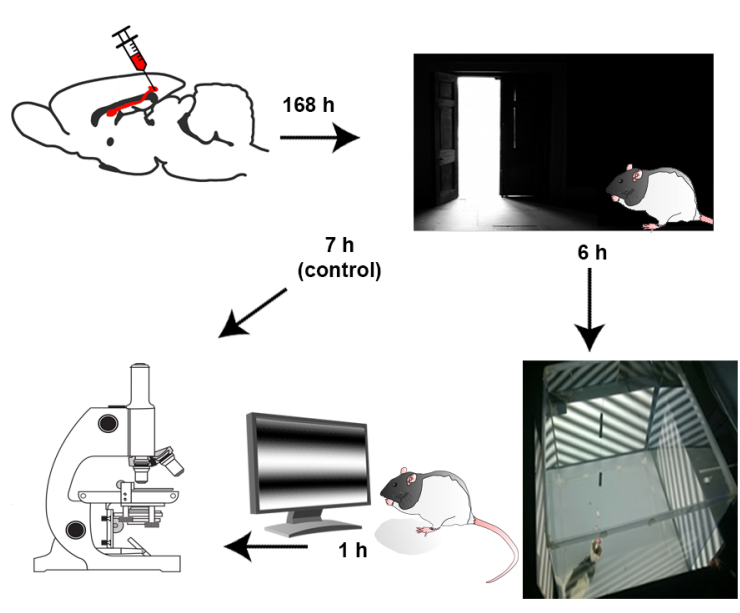

D

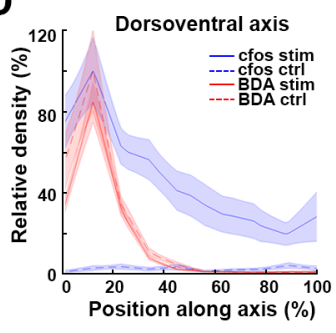

Mediolateral axis

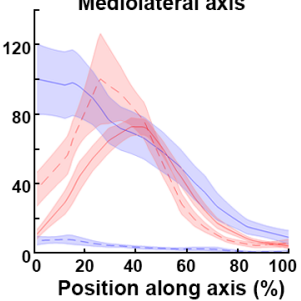

B

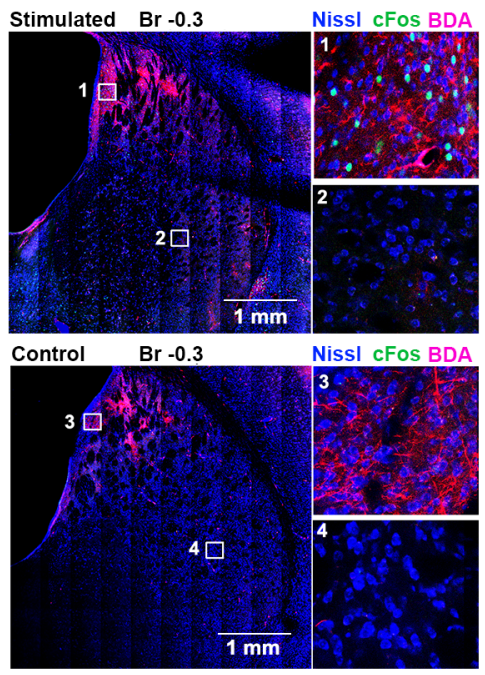

E

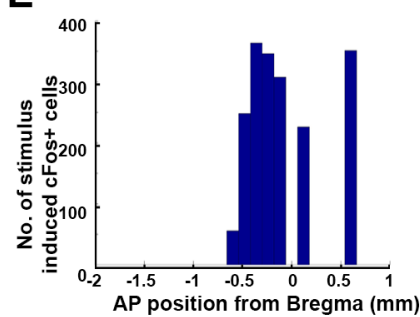

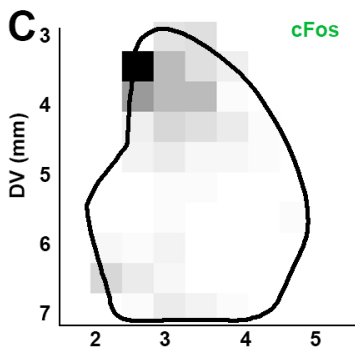
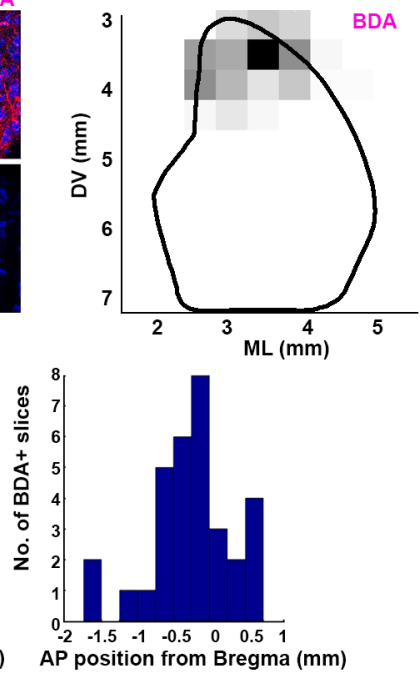

$\mathbf{F}$

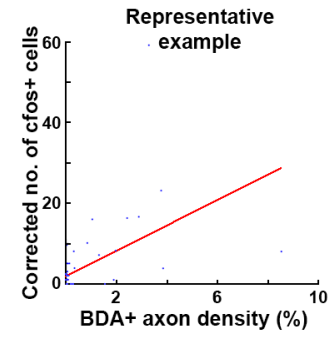

AP positi

G

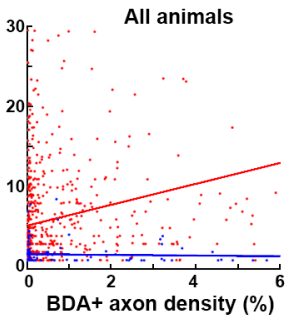

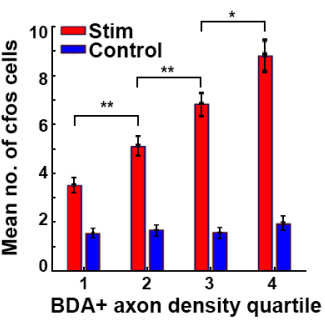

Fig 2. Colocalization of the stimulus-induced striatal cFos expression and the axon terminals from the visual cortex. (A) Experimental schematics. (B) Representative example sections of a visually stimulated (upper panels) and a control animal (lower panels). Blue, red, and green color channels are assigned to the respective fluorochromes of the Fluoro Nissl, BDA, and cFos immunohistochemistries. Insets show the magnified areas marked by rectangles on the left panels. Note the lack of cFos+ neurons on the lower (control) panels, while the anterograde BDA tract tracing from the visual cortex shows a similar axonal distribution pattern in both cases. (C) Unsupervised quantification of the striatal distribution of cFos+ neurons (top panel) and BDA+ axons (bottom panel) in a representative coronal section of a stimulated animal's brain. See also S1 Fig. (D) cFos (blue) and BDA (red) density distribution in stimulated (solid lines) and control animals (striped lines). Left and right panels show the distributions along the respective DV and ML axes of the striatum. (E) AP distribution of visual stimulation-induced cFos+ somata (left panel) and the distribution of slices with detectable FluoroGold-labeled axons ( $N=37$ slices in 9 animals). Note that the BDA+ axons target the similar AP segment of the $\mathrm{CPu}$ where the visually inducible cFos activity was present. (F) Correlation of the normalized cFos and BDA densities in a representative stimulated animal (left panel) and in all animals (right panel). The right and blue dots of the right panel and regression lines, respectively, represent data from the stimulated and sham animals. (G) Mean number of the cFos+ neurons in the striatum calculated for the 4 BDA axon density quartiles. Note that while the higher BDA density predicts higher cFos+ cell numbers in the stimulated animals (red), the cFos+ density is constant with no respect to the $\mathrm{BDA}$ distribution in the control animals (blue). AP, anteroposterior; $\mathrm{BDA}$, biotinylated dextran amine; $\mathrm{CPu}$, caudate putamen; $\mathrm{DV}$, dorsoventral; ML, mediolateral.

https://doi.org/10.1371/journal.pbio.2004712.g002

$N=490$ and 406 single positives for anti-cFos and anti-PV, respectively, and only 3 doublepositive neurons).

Automated, unsupervised algorithms were used to quantify the BDA+ axon density and the number of cFos+ neurons in $425 \times 425 \mu \mathrm{m}$ squares in each slice of the $\mathrm{CPu}$ (Fig $2 \mathrm{C}$ and 
S1 Fig). We found that axons originating from V2 neurons colocalized with the cFos+ neurons. Both the $\mathrm{BDA}+$ and cFos+ locations were restricted to the dorsal part of the $\mathrm{CPu}$. Regarding the mediolateral (ML) extent, cFos activity was present at the medial and intermediate locations, while BDA was mainly present at the intermediate (Fig 2D). The induced striatal cFos expression was present only between AP coordinates -0.7 and 0.7 from the bregma, and V2 axons targeted the same intermediate segment of the $\mathrm{CPu}$ between anteroposterior (AP) coordinates -1.7 and $+0.5 \mathrm{~mm}$ from the bregma (Fig $2 \mathrm{E}$ ).

The BDA+ axon densities in the coronal plane were spatially correlating with the cFos+ density maps of the stimulated animals (stimulated: $\mathrm{R}=0.20, p<0.005, N=696$ density pairs from 5 animals; control: $\mathrm{R}=-0.03, p=0.69, N=134$ density pairs from 4 animals, Pearson's linear correlation, Fig $2 \mathrm{~F}$ ). We concluded that the cFos expression was a result of the visual stimulation, as it was uniformly low at every location in the control animals (stimulated: $3.51 \pm 0.30,5.12 \pm 0.42,6.84 \pm 0.50,8.82 \pm 0.66 \mathrm{cFos}+$ neurons found at consecutive BDA density quartiles, $p=0.002,0.009,0.019$ for interquartile comparison, $N=4 \times 174$ density values; control: $1.55 \pm 0.19,1.69 \pm 0.19,1.59 \pm 0.22,1.94 \pm 0.28 \mathrm{cFos}+$ neurons found at consecutive BDA density quartiles, $p=0.61,0.72,0.33$ for interquartile comparison, $N=4 \times 34$ density values; two-sample $t$ test; Fig $2 \mathrm{G}$ ).

Next, to delineate the functional details of the visual responsivity of the CPu neurons, we conducted electrophysiological investigations on the rostrocaudal segment of the $\mathrm{CPu}$ that we previously identified. We recorded the activity of a total of 734 neurons using silicon electrodes ("silicon probes") in freely moving rats ( $N=42$ sessions in 6 animals) performing various behavioral and visual stimulation tasks. The recorded single units were isolated from the compound electrical activity based on their action potential waveforms, using a semiautomated clustering method $[24,25]$. Noisy units or multiunits were discarded, while clusters meeting our predefined isolation quality criteria [26] were classified as phasically firing neurons (PFNs, $N=467,63.62 \%$ of all isolated single units), fast-firing neurons (FFNs, $N=163,22.21 \%$ ), and tonically firing neurons (TFNs, $N=56,7.63 \%$ ). These classifications were based on the electrophysiological features of the corresponding waveforms and spike trains. These classes putatively match the morphological and neurochemical groups of medium spiny, $\mathrm{PV}+$ aspiny, and cholinergic aspiny neurons, respectively [4,27] (S2 Fig). In 6\% of the recorded single units, the classification was ambiguous because of their very low firing rate (FR). Those units were discarded from further analysis.

To test the responsivity of the $\mathrm{CPu}$ neurons to moving visual stimuli, animals were placed into the visual stimulation box used in the cFos expression experiments (Fig 3A). Each stimulation trial began with presenting a nonpatterned gray background for $500 \mathrm{~ms}$ (uniform screen), followed by $500 \mathrm{~ms}$ of sinusoidally modulated stationary grating pattern (stationary grating). Lastly, the pattern was gradually moved to be perpendicular to the grating's axis (moving grating) for another $500 \mathrm{~ms}$. The spatial density of the grating, the velocity, and the direction of the motion were all varied in a pseudorandom order until each parameter combination was repeated at least 150 times [9,28,29]. Neurons were considered responsive if they significantly altered their FR in response to either the stationary or the moving grating for at least 2 of the parameter combinations. Altogether, 289 neurons were tested using this paradigm (21 sessions of $\mathrm{pVis}$ stimulation), and only 25 of them were considered responsive to the self-motion-independent $\mathrm{pVis}$ stimulation (pVis+ neurons). In general, we found no systematic preference toward any of the tested directions of motion. Some neurons responded similarly to all directions, while others preferred only a few adjacent directions or two opposing ones (Fig 3B and 3C).

Regarding the spatial resolution of the grating and the velocity of the motion, neurons were clearly more sensitive to broader stripes sliding with a high velocity compared to the dense, 

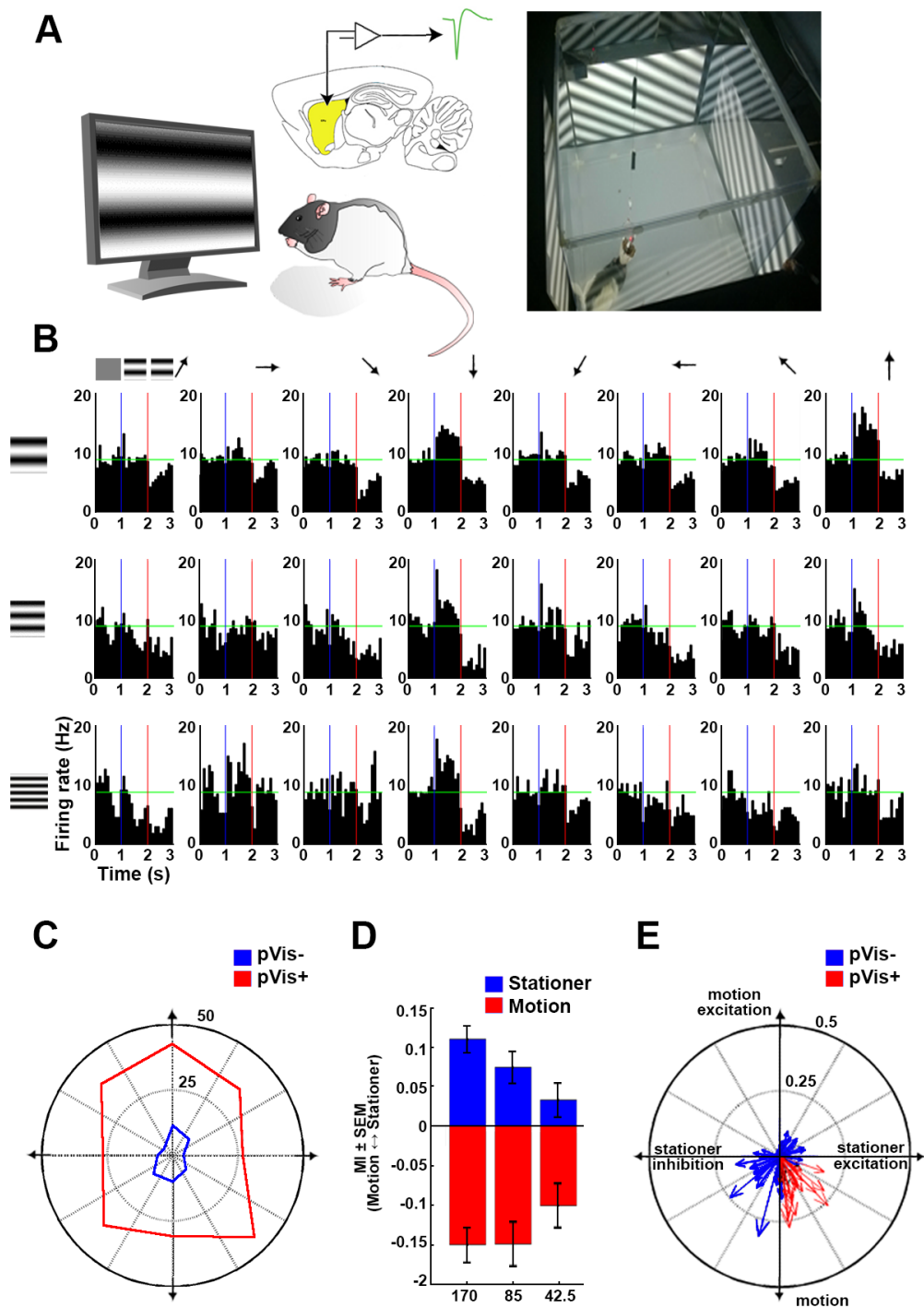

D

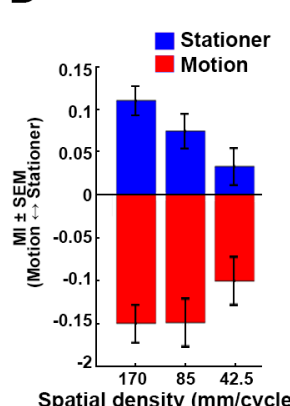

$\mathbf{E}$

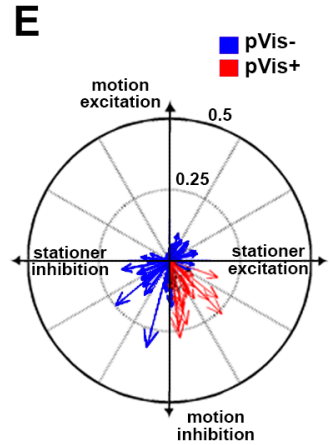

G
$\mathbf{F}$

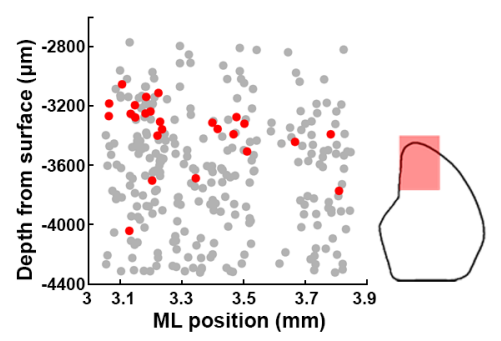

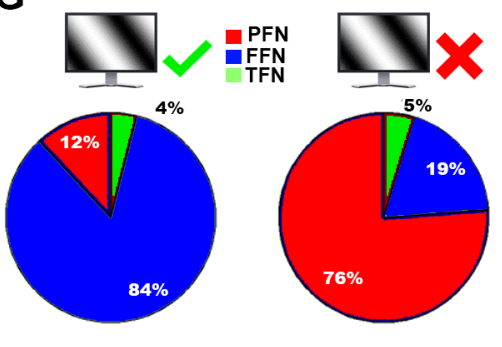

Fig 3. Self-motion-independent $p V$ is stimulation of the striatal neurons. (A) Schematics of the experimental arrangement. The striatum is marked by yellow shading. (B) Peristimulus time histograms of a pVis+ striatal neuron's responses during visual stimulation using $3 \times 8$ parameter combinations. The first third of each peristimulus time histogram represents the activity of the neuron while watching a uniform gray image. The middle and right thirds, respectively, reflect the responses during still and moving stripes. Horizontal green lines represent the spontaneous FR of the same neuron. Note that horizontally oriented still patterns evoked increased FRs, while moving stripes caused inhibition with no respect to the direction of the motion. (C) Rose plot showing the percent of pVis+ and pVisneurons responsive to the 8 different directions tested. The axes of the plot match the movement directions. Note that neither the pVis+ (red) nor the pVis- (blue) neurons expressed any obvious preference toward any motion directions. (D) Mean MIs of all pVis+ neurons during stationary (blue bars) and moving stimulation (red bars). The pVis+ 
neurons were more sensitive to low spatial and high temporal frequencies during both still and moving stimulation. (E) Response vectors of pVis+ (red) and pVis- (blue) neurons. The MIs of the 24 stimulus conditions are averaged for each cell. Still and moving MIs are assigned to the $\mathrm{X}$ and $\mathrm{Y}$ components of each response vector. Note that all $\mathrm{pVis}+$ neurons expressed stationary stimulus-induced excitation combined with motion-induced inhibition. (F) Location of the pVis+ neurons (red dots) in the coronal plane. Gray dots denote the location of the nonresponsive neurons. The red-shaded part of the inset on the right shows the area covered by the electrophysiological investigations. Note that the visually responsive cells were mainly present at the dorsomedial segment of the recorded area. (G) Distribution of cell types among the $\mathrm{pVis}+$ (left) and pVis- (right) neurons. The majority of the visually excitable neurons were FFNs, while the distribution of the other neurons followed histological proportions. FFN, fast-firing neuron; FR, firing rate; MI, modulation index; ML, mediolateral; PFN, phasically firing neuron; $\mathrm{pVis}$, passive visual; TFN, tonically firing neuron.

https://doi.org/10.1371/journal.pbio.2004712.g003

slowly shifting gratings ( $p<0.005$ for all comparisons except thick versus medium moving stripes, for which $p=0.92 ; N=200$ in each group; paired $t$ test; Fig 3D). To describe the general responsiveness of these neurons, we averaged the modulation indices (MIs) of the stationary and moving conditions (calculated against the uniform condition) across all stimulus conditions. Surprisingly, the response combinations occupied only a restricted subset of the available state space: the pVis+ neurons either increased their firing in the presence of the stationary grating or became inhibited by the moving gratings or from the combination of these two response types (Fig 3E). The pVis+ neurons were not uniformly distributed among the recorded spatial locations. They were mainly located at the mediodorsal part of the $\mathrm{CPu}$ (Fig 3F). The majority were FFNs (84\%; 12\% PFNs; and only 1 neuron was classified as a TFN). In contrast, the distribution of the putative cell types among pVis- neurons resembled the histological proportions of these classes (76\% PFN, 19\% FFN, and 5\% TFN) (Fig 3G).

The previously mentioned moving visual stimuli were presented while the animals were either standing still or when their movements were random compared to the perceived intensive visual motion (which resembled self-independent object motion). These $\mathrm{pVis}$ stimuli may be processed differently from the self-motion-induced optic flow of natural scenes, which is concordant with the other senses (e.g., the proprioception); thus, we decided to also investigate the visual responsivity of $\mathrm{CPu}$ neurons during self-motion. In contrast to experiments on head-fixed animals placed in a virtual environment, our aim was to generate a realistic visual experience that was congruent with the movements of the animals (active visual [aVis] stimulation), in order to preserve all the proprioceptive, vestibular, and exteroceptive aspects of the movements. We trained the animals to run in a linear maze back and forth for a water reward. To keep control of the visual environment seen by the rats during each run, we built the walls of the linear maze from translucent plexiglass. We placed it in a frame equipped with 2 perpendicular mirrors along the whole track (Fig 4A). Computer-generated visual scenes were projected by an LCD projector onto both the walls and the floor of the linear maze, with the help of mirrors to avoid shading by the rat. Before each trial, the projected scene was changed to either a uniformly gray ("uniform maze") or a grated pattern with similar spatial frequency and luminance as was used during the pVis stimulation ("striped maze") (Fig 4A). The "uniform" and "striped" patterns were isoluminant, and they were pseudorandomly alternated trial by trial to avoid the biasing effect of slow changes in internal brain dynamics.

In total, $685 \mathrm{CPu}$ neurons were tested in this task (in 41 sessions with 6 rats, including the neurons also tested in the $\mathrm{pVis}$ environment). In marked contrast to the small proportion of pVis+ neurons, 325 of them (62\% PFN, 26\% FFN, and 11\% TFN) displayed a significant change in their FRs in response to the striped environment (aVis+ neurons). Fig 4B shows a representative example of an aVis $+(\mathrm{FR}=0.80 \pm 0.10 \mathrm{~Hz}$ and $0.56 \pm 0.08 \mathrm{~Hz}$ in the respective uniform and striped trials, $p<0.01$, paired $t$ test, $N=20$ spatially binned FR pairs; more examples are shown on S3 Fig) and an aVis- neuron (FR $=0.53 \pm 0.08$ and $0.53 \pm 0.1 \mathrm{~Hz}$ in the 
A

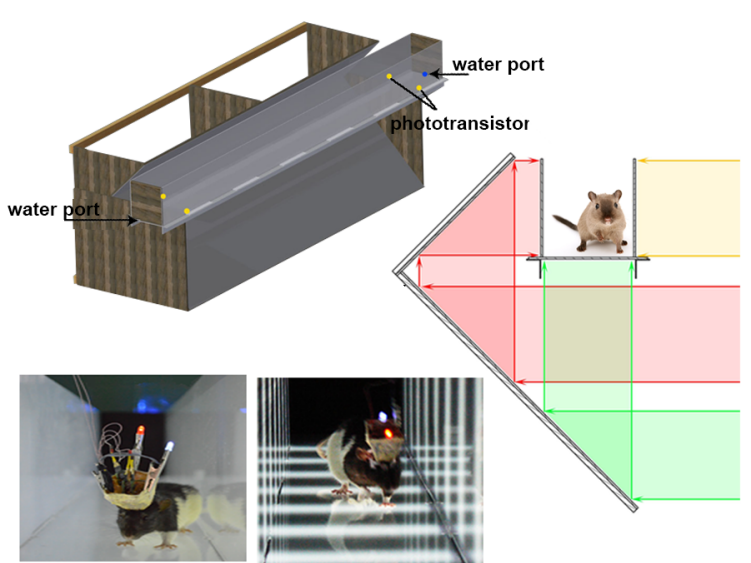

B
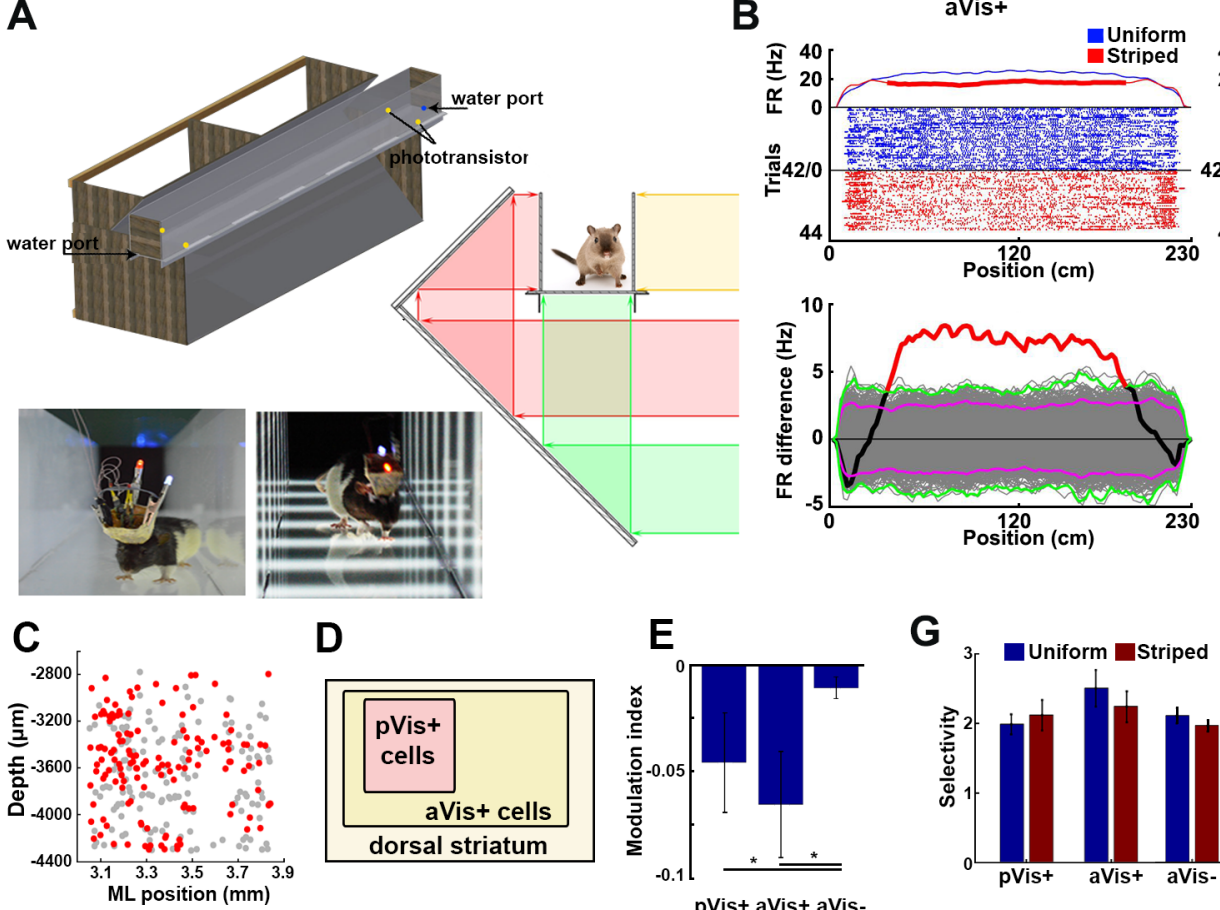

D

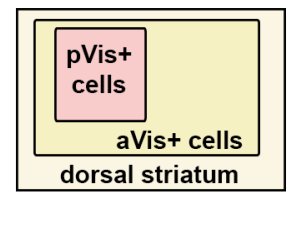

$\mathbf{F}$
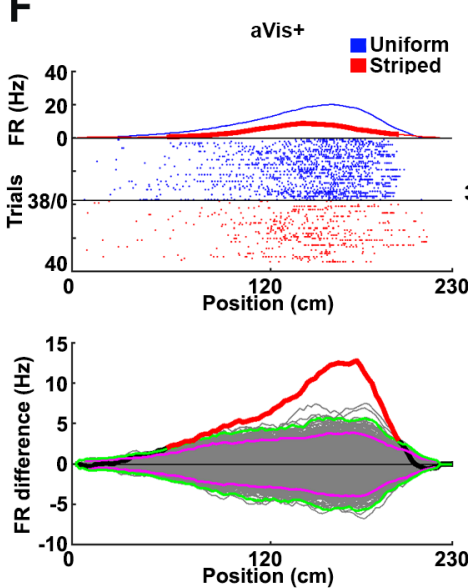

$E$

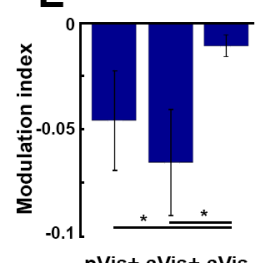

pVis+ aVis + aVis-
G

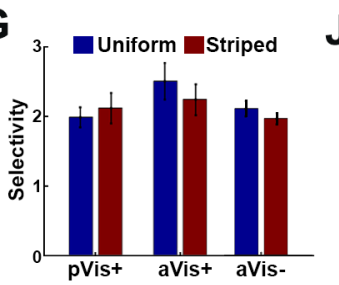

H
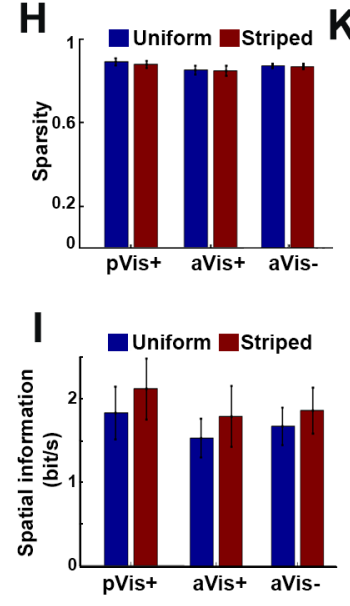

aVis-
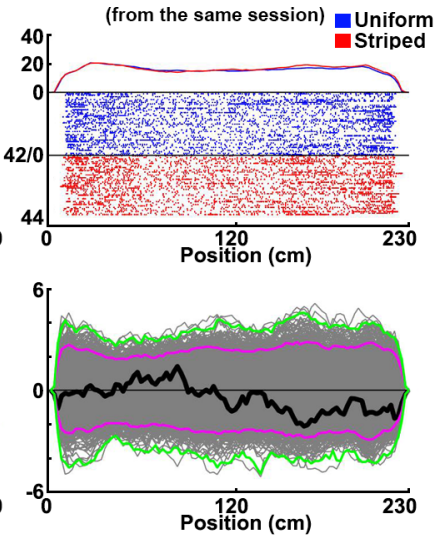

J

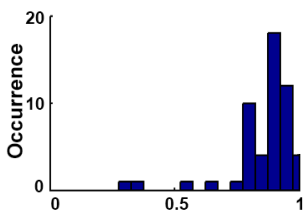

Correlation coefficient

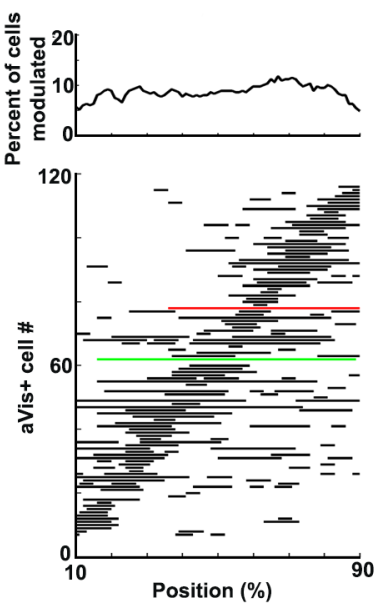

Fig 4. Self-motion-induced aVis stimulation of the striatal neurons. (A) Schematics (left) and cross section (middle) of the linear maze with a dynamically updated visual environment. The bottom panels show photographs of rats running in the uniform and the striped environment. (B) Raster plots and place-resolved FRs of a representative $\mathrm{pVis}+/ \mathrm{aVis}+$ (left, called as $\mathrm{pVis}+$ ) and an aVis-/pVis- (right, called as aVis-) neuron simultaneously recorded during the same session. The blue dots and curve represent trials during uniform maze running; the red ones show the firing patterns in the striped maze. Note that the two conditions were randomly alternated from trial to trial. For display purposes, trials are reordered by the stripedness. Lower panels show the details of the statistical tests of space-resolved visual modulation. The thick black line shows the real FR difference, while the gray curves denote the label-shuffled surrogate differences. Purple and green lines depict the "local" and "global" significance thresholds [30], respectively. Spatial locations breaking the global significance limits are marked with a thick red line. (C) Location of the $\mathrm{aVis}+$ neurons (red dots) in the coronal plane. The gray dots denote the location of the nonresponsive aVis- neurons. aVis+ neurons were distributed more uniformly in the recorded area than the pVis+ cells. (D) Venn diagram of the CPu neurons showing the set relationship of the $\mathrm{aVis}+$ and pVis+ neurons. Note that all recorded pVis+ neurons were also aVis+ at the same time. (E) Mean modulation indices of pVis+, aVis+, and $\mathrm{aVis}-$ neurons in the $\mathrm{aVis}$ environment. Note that $\mathrm{pVis}+$ neurons decreased their FRs when the rat was running in the striped maze, similar to their behavior in the pVis experiment. (F) Two representative striatal neurons with place field-like properties recorded simultaneously in an aVis session. Note that the activity of the left neuron was rate modulated by the stripedness (an aVis+ neuron), while the right example neuron was showing an almost identical spatial response profile in both the uniform and the striped conditions (aVis- neuron). Bottom panels show the details of the spaceresolved statistical tests as on panel B. (G, H, I) Spatial coding efficiency expressed as "spatial selectivity" (G), "sparsity" (H), and "spatial information" (I) of the recorded neurons in uniform (blue bars) and striped conditions (red bars). (J) Distribution of the correlation coefficients of the spatially binned FRs of neurons with place field-like activity during running in the uniform and the striped environments. The place fields were stable across both the uniform and the striped conditions, as expressed by the high spatial correlation values of the neurons with place field-like 
activity. See also S5 Fig. (K) Distribution of the spatial segments at which the FRs of the aVis+ neurons were significantly rate modulated by the perceived visual motion (bottom). Green and red lines represent the example aVis+ neurons shown on panels "B" and "F", respectively. Note that the significantly modulated segments uniformly tiled the whole extent of the maze (top plot). aVis, active visual; FR, firing rate; ML, mediolateral; pVis, passive visual.

https://doi.org/10.1371/journal.pbio.2004712.g004

respective uniform and striped trials, $p=0.76$ ) recorded simultaneously with its aVis+ peer. The aVis+ neurons were not restricted to the most superficial and medial part of the mediodorsal $\mathrm{CPu}$. Instead, they were distributed more uniformly (Fig 4C).

Importantly, all pVis+ neurons were also responsive in this task. Thus, the pVis+ cells can be considered as a subgroup of the aVis+ neurons (Fig 4D). For simplicity, from here on we will refer to the double responsive (i.e., $\mathrm{pVis}+\mathrm{aVis}+$ ) neurons as $\mathrm{pVis}+$ neurons, and those cells which were only responsive in the aVis environment but not in the pVis one (i.e., pVis-/aVis+ neurons) as aVis+ neurons.

The firing pattern of the $\mathrm{pVis}+$ neurons in the uniform maze was more deterministic with regard to their activity in the striped maze, compared to the $\mathrm{pVis}-$ neurons $(\mathrm{R}=0.77 \pm 0.02$ and $0.65 \pm 0.02$ for $\mathrm{pVis}+$ and $\mathrm{pVis}-$ neurons, respectively; Pearson's linear correlation between uniform and striped runs, $p=0.032$, two-sample $t$ test, S4 Fig). In agreement with their behavior during the $\mathrm{pVis}$ stimulation experiment, the pVis+ neurons decreased their FRs while the rats were running in the striped linear maze.

The visual modulation of the aVis+ neurons in this task was similar to the response profiles of the pVis+ neurons ( $p=0.047$ for $\mathrm{pVis}+$ versus aVis-, and 0.041 for aVis+ versus aVis- comparisons, $\mathrm{MI}=-4.7 \% \pm 2.3 \%,-6.9 \% \pm 2.7 \%$, and $-1.0 \% \pm 0.5 \% ; N=12,133$, and 50 , respectively, for the pVis+, aVis+, and aVis- neurons, two-sample Kolmogorov-Smirnov test; Fig $4 \mathrm{E})$. This result confirmed the visual motion-induced inhibition observed in the passive stimulation task.

The dorsomedial part of the intermediate striatum is known to integrate highly heterogeneous, multimodal inputs in mice [31]. We were therefore interested if pVis+ and/or aVis+ neurons also code context- or location-dependent features as suggested by earlier studies $[32,33]$. A significant number of neurons showed spatial location-selective activity, similar to the place cells [33,34] of the hippocampal formation (36\% of the pVis+, $20 \%$ of the aVis+, and $17 \%$ of the aVis- neurons). All of these neurons had one single place field similar to the proximal CA1 and CA $3 c$ regions of the hippocampus [35]. However, their "place fields" were relatively large, usually spanning over more than one-third of the track (Fig 4F and S3 Fig).

The FRs of these "place cell-like" neurons were rate modulated by the presence of striped patterns to a similar extent as the activity of those that were active at every location. The spatial coding ability of the aVis+ neurons was slightly (but not significantly) better than either the pVis + or the aVis- neurons and was not influenced by the presence of the striped pattern (selectivity of pVis + cells: $1.98 \pm 0.14$ and $2.11 \pm 0.21$; aVis + cells: $2.46 \pm 0.3$ and $2.33 \pm 0.28$; aVis- cells: $2.11 \pm 0.11$ and $1.96 \pm 0.09$; sparsity of pVis+ cells: $0.88 \pm 0.01$ and $0.87 \pm 0.01$; aVis + cells: $0.85 \pm 0.02$ and $0.85 \pm 0.02$; aVis- cells: $0.87 \pm 0.01$ and $0.87 \pm 0.01$; spatial Information of pVis + cells: $1.83 \pm 0.32$ and $2.12 \pm 0.36$; aVis+ cells: $1.63 \pm 0.25$ and $1.90 \pm 0.39$; aVis- cells: $1.78 \pm 0.24$ and $1.98 \pm 0.29$, in the uniform and striped mazes respectively; $p>0.05$ for all comparisons, paired $t$ test across conditions, and Kolmogorov-Smirnov test across neurons; $N=21$ pVis+, 45 aVis+, 31 aVis- FFNs [35]; Fig 4G-4I).

The locations and extent of the place fields were highly correlating in both the uniform and the striped trials. This suggests that the animals considered the two conditions spatially identical despite the different visual percepts (mean Rho $=0.86 \pm 0.13 ; p<0.05$ in 51 of 53 neurons, Pearson's linear correlation of location-resolved FRs in the two conditions, 20 spatially binned 
FR pairs per neuron, Fig 4J and S5 Fig), and the perceived visual motion caused only a rate remapping [36,37] but not a location remapping of the "place fields." The spatial locations where the stripedness significantly altered the neuronal activity were determined by comparing the induced firing pattern differences to surrogate datasets (Fig 4B and S3 Fig). Of the aVis+ cells, 124 met the spatially resolved significance criteria (see Materials and methods). The significantly modulated segments of the space-resolved firing patterns uniformly tiled the whole extent of the linear maze; $8.51 \% \pm 0.38 \%$ of the $124 \mathrm{aVis}+$ neurons were modulated at any spatial location ( $p=0.76, N=2,444$, chi-squared goodness-of-fit test against uniform distribution, Fig $4 \mathrm{~K})$.

The visual modulation of the firing patterns was stronger when faster visual motion was perceived (i.e., faster running speeds), similarly to the spatiotemporal tuning found in the $\mathrm{pVis}$ task (Rho $=-0.46, p<0.001$, Pearson's linear correlation of the instantaneous velocity and the visual MIs in $N=304$ spatial bins from 28 sessions, S6A Fig).

Only a small fraction of the visually responsive neurons modulated their FRs as a function of the instantaneous running speed ( $p<0.05$, Pearson's linear correlation; number of modulated neurons $=3$ of $25 \mathrm{pVis}+, 20$ of $221 \mathrm{aVis}+, 26$ of $233 \mathrm{aVis}-$ ). The velocity of the animals was identical in the two environments $(83.3 \pm 18.9 \mathrm{~cm} / \mathrm{s}$ versus $85.0 \pm 12.3 \mathrm{~cm} / \mathrm{s}, n=718$ and 649 for uniform and striped conditions, respectively; $p=0.15$, two-sample Kolmogorov-Smirnov test; S6B Fig).

Although the overall brightness of the "uniform" gray and the black and white "striped" conditions was identical, an alternative explanation of the FR modulation of the aVis+ cells may be that the striped pattern by itself could influence the neuronal activity, either by inducing a different contextual percept or by its local brightness fluctuations. To exclude these alternatives, we performed a set of complementary experiments and analyses.

The rats perceived a relatively stationary representation of the striped and the blank patterns while they stayed at the reward zones (i.e., the last $10 \mathrm{~cm}$ of the maze at each end). We compared the firing patterns of the aVis+ cells during these segments to decide if the two contexts induced the FR modulations. The animals spent comparable time in the reward zones $(23.11 \pm 1.88 \mathrm{~s}$ and $26.46 \pm 2.14 \mathrm{~s}, N=1,303$ and 1,343 trials for uniform and striped conditions, respectively, $p=0.24$, two-sample $t$ test, S6C Fig), and the mean FRs of the aVis+cells were almost identical (mean FRs $=9.01 \pm 0.89 \mathrm{~Hz}$ and $8.99 \pm 0.88 \mathrm{~Hz}$ for uniform and striped conditions, respectively, $p=0.83, N=325$ neurons, paired $t$ test, S6D Fig). By comparing the neuronal activity on a cell-by-cell and trial-by-trial basis, only 36 of the 325 aVis+ neurons showed significantly different FRs in the two conditions $(p<0.05$, two-sample $t$ test on the per-trial firing patterns for each neurons, S6E Fig).

We also tested if the aVis + neurons were sensitive to changes of brightness. First, to check their sensitivity for global changes, we exposed the rats to $0.5 \mathrm{~Hz}$ blinking light in the $\mathrm{pVis}$ environment. S6F Fig shows 2 representative neurons modulated by the global luminance change ( $p<0.001$ in both cases, $N=50$ trials). Eleven and 2 of the 20 tested pVis + cells, 5 and 11 of the $111 \mathrm{aVis}+$ cells, and 7 and 12 of the $124 \mathrm{aVis}-$ neurons, respectively, responded with excitation and inhibition for the luminance increase $(p<0.05$, paired $t$ test, $N=50$ trials for each neuron; S6F and S6G Fig).

Second, we analyzed FRs in the linear maze stripe cycle by stripe cycle to check if the aVis+ neurons display phasic responses to the gradually changing brightness of each stripe. The aVis+ neurons were not sensitive to the local luminance changes of the black and white stripes. The FRs were nearly uniform at locations with low and high local luminance in the striped condition $(p=0.23$ and $0.45, N=193$ and 358 spikes for the uniform and the striped conditions, respectively; Rayleigh test; $\mathrm{S} 6 \mathrm{H}$ Fig shows the stripe phase-resolved firing patterns of a representative example neuron). For all aVis+ neurons, the circular MIs of the FR patterns 
A

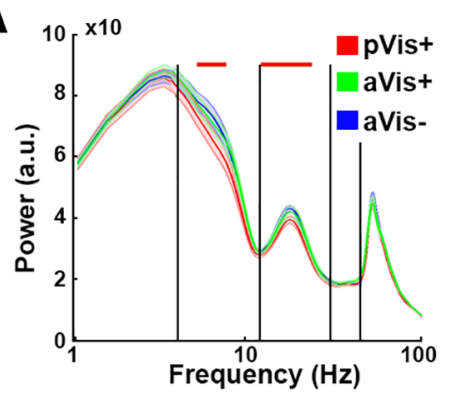

B
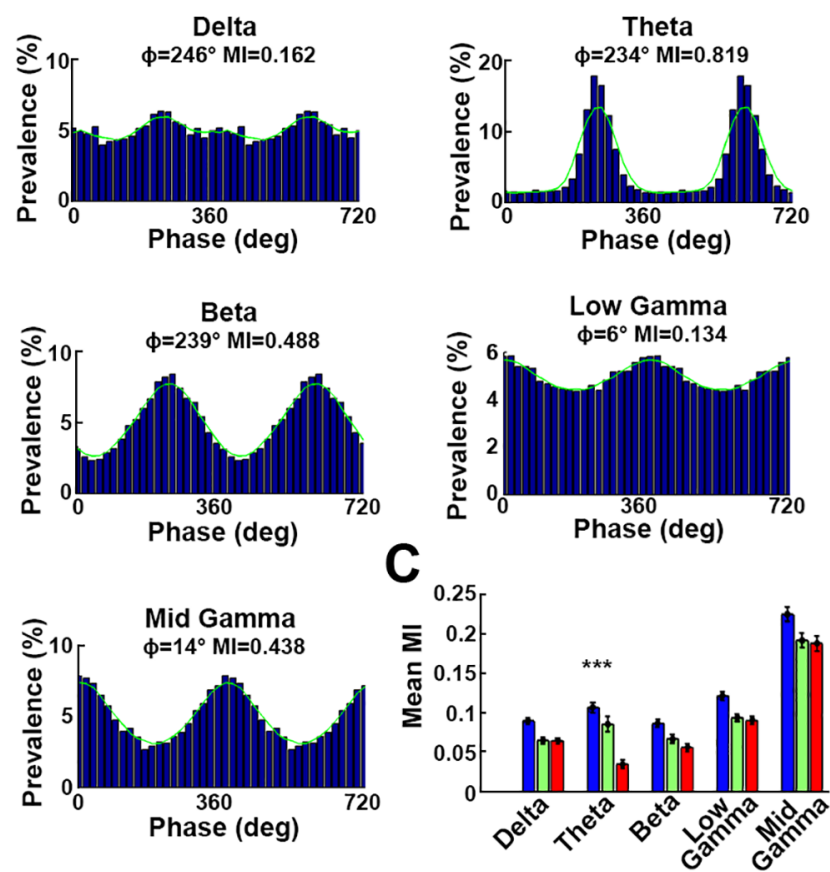

Fig 5. Phase modulation of the recorded CPu neurons by the characteristic frequency bands of the striatal LFP. (A) Mean LFP spectral power at locations of the pVis+ (red), aVis+ (green), and pVis-/aVis- somata (blue). The theta and beta powers were significantly weaker at locations where $\mathrm{pVis}+$ neurons were present (the red horizontal lines show the significant segments). (B) Respective phase coupling of a representative neuron to the delta, theta, beta, lowgamma, and high-gamma bands. The mean preferred phase values and MIs are shown above each plot. Green curves mark the smoothed distributions. (C) Mean MIs of the pVis-/aVis- (blue), aVis+ (green), and pVis+ (red) neurons. The pVis+ neurons were less coupled to the phase of the theta and beta band oscillations. The color codes are the same as on panel A. a.u., arbitrary unit; aVis, active visual; $\mathrm{CPu}$, caudate putamen; LFP, local field potential; $\mathrm{MI}$, modulation index; $p$ Vis, passive visual.

https://doi.org/10.1371/journal.pbio.2004712.g005

were similar in the "striped" and the "uniform" trials ( $p=0.86$, two-sample $t$ test, mean $\mathrm{MI}=13.22 \% \pm 1.50 \%$ and $13.61 \% \pm 1.82 \%, N=93$ and $78 \mathrm{aVis}+$ neurons with nonuniform circular distribution in the uniform and the striped conditions, respectively; S6I Fig).

These observations led us to conclude that the FR change of the aVis+ neurons between the two applied conditions was induced by the perceived visual motion, as all other aspects were kept and were perceived unchanged (i.e., trajectory, reward, global luminance, and other contextual cues).

The neurons of the $\mathrm{CPu}$ were generally phase locked to the characteristic frequency bands of the local field potential (LFP, Fig 5A and 5B, S7 Fig) recorded in the dorsomedial CPu. In general, the $\mathrm{pVis}+$ and aVis+ neurons were more weakly coupled to these oscillations than 
their pVis-/aVis- peers (mean MIs, delta: $10.84 \pm 0.50$ versus $7.89 \pm 0.50$ and $7.85 \pm 0.41$; theta: $12.89 \pm 0.76$ versus $10.37 \pm 1.16$ and $4.29 \pm 0.65$; beta: $10.48 \pm 0.58$ versus $8.12 \pm 0.65$ and $6.80 \pm 0.58$; low-gamma: $14.64 \pm 0.64$ versus $11.36 \pm 0.53$ and $10.93 \pm 0.59$; high-gamma: $26.96 \pm 1.10$ versus $23.02 \pm 1.12$ and $22.57 \pm 1.12$ for $\mathrm{pVis}-/ \mathrm{aVis}-$ versus aVis + and $\mathrm{pVis}+$ neurons, respectively). This difference in phase coupling was the most prominent in the theta band ( $p<0.001, n=145$ and 25, Kolmogorov-Smirnov test, Fig 5C).

To check if the anatomically identified cortical regions indeed play a role in shaping the activity of the visual motion-sensitive $\mathrm{CPu}$ cells, we lastly set out to investigate their functional connectivity. However, confirming the direct monosynaptic cell-to-cell interactions between the visual cortex and the $\mathrm{CPu}$ neurons in intact animals is like looking for a needle in a haystack. Cross-correlation-based analyses are very unreliable with sparsely firing neurons because of the low number of spikes, even if extensive recordings are done for many hours. Optogenetics-based transsynaptic excitation/inhibition is also problematic because of the unknown synaptic transmission efficacy. Moreover, $\mathrm{V} 1$ is known to project only to a very thin medial strip of the $\mathrm{CPu}$ along its ventricular wall [20]. This is extremely difficult to investigate using electrophysiological approaches in freely moving animals. Because of these constraints, we employed two alternative approaches.

First, we implanted bipolar-stimulating electrodes into area V2 (AP $-4.5 \mathrm{~mm}$; ML $2.8 \mathrm{~mm}$; dorsoventral [DV] 0.5 and $2 \mathrm{~mm}$ ) of 2 animals. In a subset of sessions, we delivered short electrical pulses ( $N=21$ sessions) to functionally verify the previously confirmed long-range anatomical connection (Fig 6A). The electrically evoked, complex LFP deflections had 3 distinct components whose latency and duration matched with the single unit-evoked responses: a narrow, precisely timed monosynaptic deflection was followed by a wider (presumably disynaptic) one and an even longer polysynaptic waveform. In contrast, at cortical

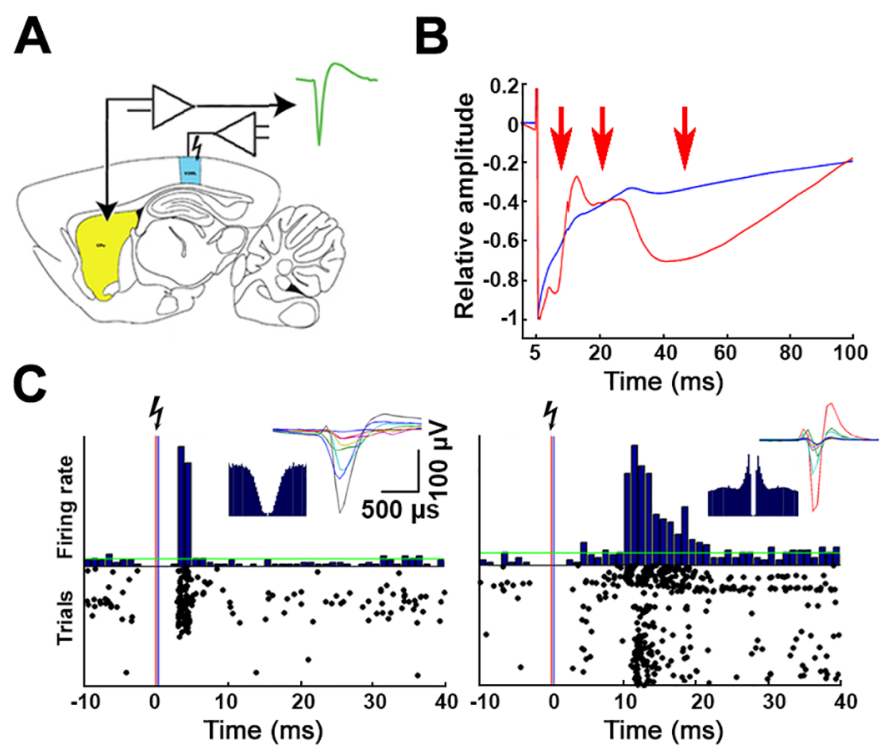

Fig 6. Entrainment of the CPu activity by electrical stimulation of the V2. (A) Schematics of the electrical stimulation. The sites of the cortical stimulation and the striatal recordings are respectively marked by blue and yellow colors. (B) Representative striatal (red) and a control cortical (blue) mean LFP traces as a response to V2 electrical stimulation. Note the 3 remarkable evoked deflections in the striatal LFP (red arrows), which represent the aggregate population activity of the mono-, di-, and polysynaptically activated CPu neurons. (C) Representative peristimulus time histograms and raster plots of a monosynaptically (left panel) and a polysynaptically (right panel) entrained $\mathrm{CPu}$ neuron during electrical stimulation of the V2. The insets show the waveforms and autocorrelograms of each example neuron. $\mathrm{CPu}$, caudate putamen; LFP, local field potential; V2, secondary visual cortex.

https://doi.org/10.1371/journal.pbio.2004712.g006 
locations above the $\mathrm{CPu}$, only a single monotonic deflection attributed to the volume-conducted stimulus artefact was present (Fig 6B). The brief electrical pulses evoked spiking in 32 out of $289 \mathrm{CPu}$ neurons, either with very short response latency (4-6 ms, representing a monosynaptic input) or through a longer multisynaptic pathway (Fig 6C). The group of neurons responsive to electrical stimulation of area $\mathrm{V} 2$ and the group of $\mathrm{pVis}+$ neurons were mainly overlapping; 20 of 25 (80\%) pVis+ neurons were also sensitive to electrical stimulation, while the same applied to only 12 of $252(6 \%)$ pVis- cells. Consequently, the intrastriatal distributions of these groups were similar; they were also mainly located at the mediodorsal part of the $\mathrm{CPu}$.

As a second approach, we implanted an additional 32-channel silicon probe into area V2 in 2 animals. We recorded both single units and LFPs while the animals were exposed to the aVis stimulation protocol in the linear maze (Fig 7A). The frequency spectra of the LFPs recorded at the $\mathrm{CPu}$ and at $\mathrm{V} 2$ were identical in the striped and uniform trials (except $80-90 \mathrm{~Hz}$ in V2, Fig $7 \mathrm{~B})$. This suggests that the visual environment does not alter the gross activity of these structures. Still, V2 activity has a role in shaping the activity of the striatum, and its influence is approximately $10 \%-15 \%$ weaker in the striped environment than in the uniform trials. This was shown by the Granger causality between the $\mathrm{V} 2$ and $\mathrm{CPu}$ broadband and the low-gamma filtered LFPs (V2 $\rightarrow$ CPU: $\mathrm{F}_{\text {broadband }}=15.58 \pm 0.22$ and $13.27 \pm 0.2 ; \mathrm{F}_{\text {low-gamma }}=3.63 \pm 0.06$ and $3.32 \pm 0.06$; CriticalValue broadband $_{1}=3.13 \pm 0.008$ and $3.25 \pm 0.008$; CriticalValue $_{\text {low-gamma }}=$ $3.63 \pm 0.008$ and $3.67 \pm 0.008 ; N=3,424$ and 4,096 trials in the uniform and the striped conditions, respectively; $p<0.001$ for both the broadband and low-gamma band comparison of the F-values of all trials; two-sample $t$ test; Fig $7 \mathrm{C}$ ). Regarding the information flow from the $\mathrm{CPu}$ to V2, the analysis could not reveal significant causality in the gamma band; however, the broadband causality was maintained. We did not find any difference between the uniform and striped conditions in this case $\left(\mathrm{CPu} \rightarrow \mathrm{V} 2: \mathrm{F}_{\text {broadband }}=15.71 \pm 0.24\right.$ and $14.85 \pm 0.4$; $\mathrm{F}_{\text {low-gamma }}=1.98 \pm 0.08$ and $1.81 \pm 0.25$; CriticalValue broadband $=3.01 \pm 0.008$ and $3.01 \pm 0.008$; CriticalValue $_{\text {low-gamma }}=3.84 \pm 0.008$ and $3.84 \pm 0.008 ; N=3,424$ and 4,096 trials in the uniform and the striped conditions, respectively; $p=0.21$ and 0.24 for the broadband and lowgamma band comparison of the F-values of all trials, respectively; two-sample $t$ test; Fig 7C).

The high level of causality of the broadband signals in the $\mathrm{CPu}-\mathrm{V} 2$ direction calls for the cautious interpretation of the Granger causality analysis [38]. Traditional correlation and coherence analyses cannot reveal sparse, package-like increases in the synchrony of discrete frequency bands, especially if they are lagging. This is because the asynchronous, uncorrelated majority of the signals hide it. To overcome this bottleneck, we employed a time and frequency-resolved cross-coherence estimation. This is particularly sensitive to lagging, intermittent changes in synchrony, such as "bursty" transmissions (S8 Fig). Using this method, we found that there are transient oscillations in the mid-gamma band that appear coherently in both V2 and the $\mathrm{CPu}$ with a constant time lag (V2 leading with approximately $30 \mathrm{~ms}$, Fig 7D). A similar but less coherent delayed pattern was present between $\mathrm{V} 2$ and the motor cortex (recorded above the $\mathrm{CPu}$ as control, Fig 7E).

In contrast to the V2-motor cortex coherence, which was similar both during running in the uniform and the striped mazes (correlation index $=0.058 \pm 0.004$ and $0.054 \pm 0.006$, respectively, for the uniform and striped conditions; $p=0.37 ; N=64$ recording-site pairs), the V2 coherence with the $\mathrm{CPu}$ became significantly weaker when the striped pattern was present (correlation index $=0.11 \pm 0.004$ and $0.07 \pm 0.003$, respectively, for the uniform and striped conditions; $p<0.001 ; N=96$ location pairs, Fig 7F). To confirm if the decreased gamma band coherence also has an effect in shaping the striatal unit activity, we tested the LFP phase coupling of the striatal spike trains of the aVis + neurons. The strength of the phase coupling to local LFP was identical in the uniform and the striped conditions. In contrast (as predicted by 
A
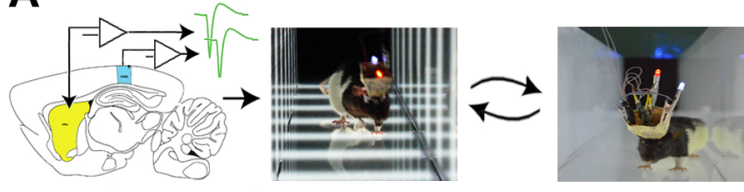

B

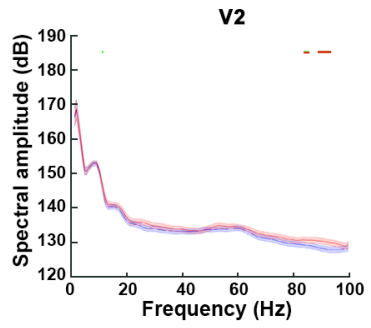

C

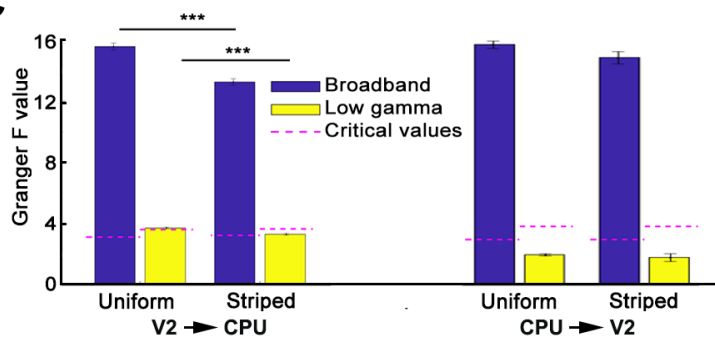

D Uniform V2 vs $\mathrm{CPu} \quad$ Striped
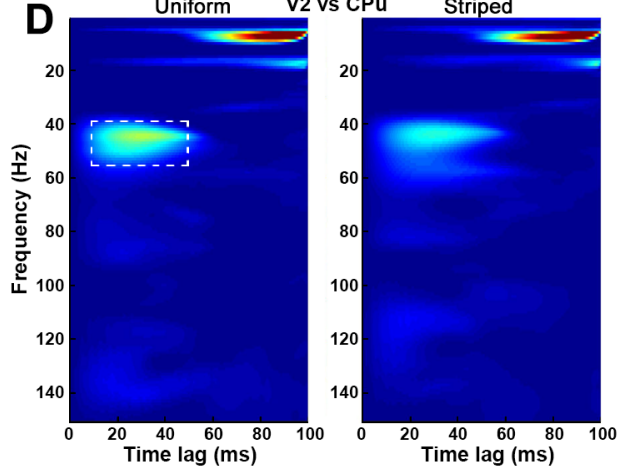

$\mathbf{E}$

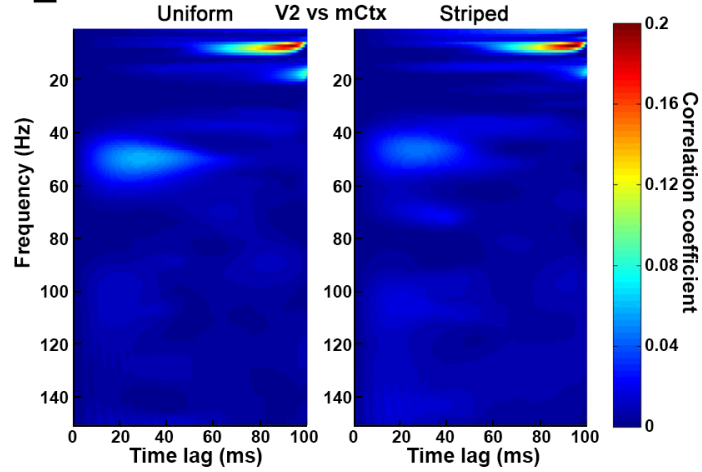

G

$\mathbf{F}$

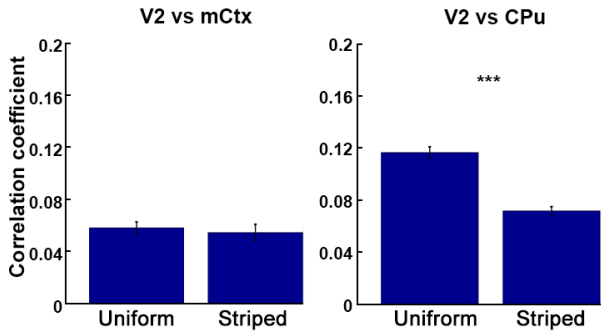

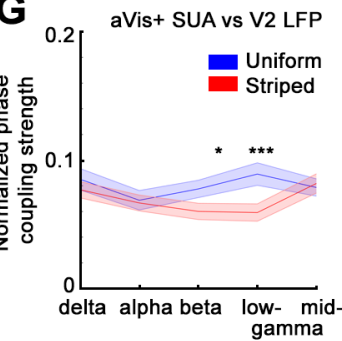

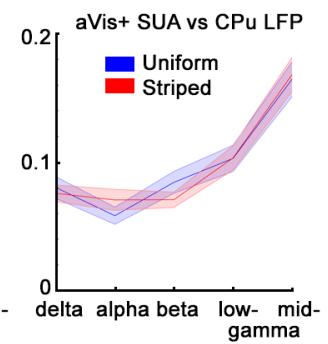

Fig 7. Activity coupling between V2 and the CPu. (A) Experimental schematics showing the simultaneous recording of the LFPs and SUA in both the V2 and the dorsomedial CPu. (B) Mean raw spectra of the V2 (left) and CPu (right) are almost identical when the animals are running in both the uniformly gray (blue lines) and the striped (red) environments (mean power spectra of $N=30$ and 175 LFP sessions, respectively). The only significant difference was detected in the high-gamma band of the V2 (red horizontal lines). (C) V2 LFP Granger causes striatal LFP oscillations both in the broadband (left panel, blue) and low-gamma filtered signals (left panel, yellow). Note the significant decrease of the causality strength in the striped environment compared to the uniform trials. Right panel denotes the results of the causality analysis in the striato-cortical direction. (D) Average time and frequency-resolved coherence maps across all sessions and animals for trials in the uniform (left) and striped (right) environments. Note the presence of the high-coherence domain between 40 and $50 \mathrm{~Hz}$ with a temporal lag of 20-40 ms (V2 leading). (E) Same as in (D) when comparing V2 LFP with the LFP of the mCtx serving as a control. (F) Comparison of the maximal coherence values between $40-55 \mathrm{~Hz}$ and $5-50$ $\mathrm{ms}$ (marked by a dashed white box on (D)). The left and right panels respectively compare the coherences of the mCtx versus $\mathrm{V} 2$ and $\mathrm{CPu}$ versus V2 during running in both striped and uniform environments. $(\mathrm{G})$ Phase coupling of aVis+ $\mathrm{CPu}$ neurons to various frequency bands of the V2 (left) and the striatal (right) LFP during uniform (blue) and striped (red) trials. Note the stripednessinduced decoupling of the aVis+ cells from the V2 beta and low-gamma bands. aVis, active visual cortex; $\mathrm{CPu}$, caudate putamen; LFP, local field potential; mCtx, motor cortex; SUA, single-unit activity; V2, secondary visual cortex.

https://doi.org/10.1371/journal.pbio.2004712.g007

the LFP coherence analysis), coupling to the distal V2 LFP was decreased in the beta and lowgamma bands when the animal was running in the striped environment (Fig 7G). These findings suggest the presence of a population-level communication "channel" between V2 and the dorsomedial $\mathrm{CPu}$, which is influenced by the visual environment. 


\section{Discussion}

We found a small, distinct group of the dorsomedial striatal neurons that are responsive to both self-motion-independent (passive) and self-motion-congruent (active) visual stimuli (pVis+ neurons). In addition, there is a larger subset of striatal cells that only sense visual motion, when congruent locomotion is simultaneously present (aVis+ neurons). These findings emphasize the importance of investigating visual motion-related sensory processing in freely moving animals instead of anesthetized or head-fixed preparations $[9,22,39]$. The perceptual capacity represented by these two cell groups may support survival. They allow for the recognition of incongruently moving objects (such as prey). At the same time, due to the selfmotion of the observer, they also provide reafferentation about the continuously changing visual environment. Even though the two functional groups we identified in this study shared many electrophysiological properties, our results suggest that $\mathrm{pVis}+$ neurons were overwhelmingly FFNs.

\section{Identification of striatal cell types from extracellular recordings}

Despite their extensive interconnections, the $\mathrm{CPu}$ has a very distinct cytoarchitecture from the cortex. The internal circuitry of the $\mathrm{CPu}$ is shaped almost exclusively by GABAergic inhibitory neurons, plus a few cholinergic neuromodulator cells. Of these GABAergic cells, $95 \%$ are medium spiny neurons forming the functional class of PFNs, while the cholinergic cells are acting as TFNs [40]. The remaining $5 \%$ of aspiny GABAergic cells were recently described as a mixture of at least eight morphologically, neurochemically, and electrophysiologically different classes of cells [41-43]. The FFN neurons in this study are most likely PV+, fast-spiking interneurons $[27,43,44]$. However, the functional classification of the CPu neurons can only putatively and cautiously be matched to the known neurochemical classes. We were able to isolate significantly fewer PFNs and more FFNs (similar to Thorn and Graybiel [45]), as was expected from the results of histological studies with unbiased sampling. PFNs are particularly sparsely firing neurons. Thus, they are the most vulnerable to forced exclusion due to numerical approximation issues or accidentally skipped detection.

\section{Immediate early gene (IEG) expression in the CPu by visual stimuli}

Immunohistochemistry allows for the exact identification of the histochemical cell types in the $\mathrm{CPu}$. However, conclusions about their visual responsivity based on their cFos expression alone should be interpreted with caution. Although the $\mathrm{CPu}$ is known to express IEGs in response to cortical stimulation [46], they are not uniformly expressed among different cell types [47]. They can only report increases of FRs reliably [47]. Lastly, it is not possible to distinguish between mono- and polysynaptic activation. We chose to detect cFos over JunB, FosB, or other IEGs for two reasons: first because of its very low constitutive expression in the $\mathrm{CPu}$ and second because it is expressed by most cell lines upon stimulation [46]. pVis+ neurons were inhibited by visual motion, which cannot explain the induced cFos expression. We speculate that it was either induced by the blinking light or the stationary grating parts of the stimulation sequence (i.e., more than half of the pVis+ neurons were excited by the change of brightness, and they were also typically excited by the stationary gratings). An alternative explanation is that these neurons were activated through a disinhibition, as the $\mathrm{pVis}+$ neurons were mainly FFNs, but the cFos+ cells were PV-. In this latter case, the cFos+ cells could be mainly medium spiny neurons, which are known to receive inhibitory innervation from the FFNs (putative PV+ fast-spiking interneurons [43]). 


\section{Origin and processing of the visual information in the dorsomedial striatum}

Hunnicutt and colleagues [31] reported that neurons of the dorsomedial part of the mouse striatum respond to multiple sensory modalities and that they integrate auditory and visual inputs with emotional and contextual information. The exact drivers of the visual features of the $\mathrm{CPu}$ are not clear. We found that aVis+ neurons were sensitive to global (but not local) luminance changes. This finding is in agreement with earlier reports on the large receptive fields of striatal cells spanning almost the whole visual field in cats [29]. These results suggest that the striatum receives a preprocessed "global percept" of the visual environment, instead of a partial pixelated representation.

The cortical discrimination of self-motion-induced visual motion and object motion is generally linked to higher-order visual areas along the dorsal stream of the visual cortex, such as the middle temporal and dorsal medial superior temporal area $[39,48,49]$. V6 also seems to be able to subtract self-motion-induced dislocations from the visual percept to enhance object motion detectability $[48,50]$. However, earlier stages of the visual pathway (including V1) can already detect visual motion [51], process features of the motion [52], and even integrate visual motion and locomotion percepts [53]. Upstream stages (such as V2 or V3) inherit these capacities [54] and can serve as a common source for motion-related information to both V5 and V6 pathways [48]. The anterolateral part of area 18a (the lateral extrastriate cortex) of the mouse (which is the homologue of the lateral secondary visual cortex [V2L] and mediolateral secondary visual cortex [V2ML] in the rat [55]) is also linked to the processing of self-motion cues [56]. These V2 neurons prefer lower spatial frequencies compared to V1 and have relatively large receptive fields [54].

Visual motion-sensitive $\mathrm{CPu}$ neurons have similar preferences. An earlier report [9] found that caudate nucleus neurons of the cat prefer similar low spatial and high temporal frequencies to what we report here regarding the $\mathrm{pVis}+$ neurons of rats. A direct connection between the visual cortex and dorsocaudal striatum was also confirmed by a few tracing studies in rats and monkeys $[20,23,57]$. The inhibition of the FFNs by the visual motion in this study suggests an indirect cortical effect through other striatal interneurons (signal inversion). However, this contradicts the direct electrical excitability of the pVis+ neurons. An alternative explanation may be provided by the already negative coding scheme of the corticostriatal afferents from the complex neurons of the V1 [58-60] or, for example, the so-called D cells of the feline extrastriate cortex. These neurons maintain high spontaneous FRs that are decreased when visual motion is perceived [61]. Such decrease in the cortical drive may explain the motion-induced decrease of spiking in the pVis+ neurons, as well as the monosynaptic cortical entrainment that we report here.

Our findings regarding the selective cortical excitability of the pVis+ (but not the aVis+ cells) and their different intrastriatal distribution offer the idea of multiple parallel visual streams as drivers. However, our results only partially support this concept. The more ventral and lateral parts of the dorsomedial $\mathrm{CPu}$ (where the aVis+ neurons are also located) did not receive direct $\mathrm{V} 2$ input, but the similar response profile in the striped maze indicates that the pVis+ neurons are more likely to be a subtype of the aVis+ group. Nevertheless, as one candidate for the alternative innervation, the lateral-posteromedial nucleus of the thalamus projects to the same striatal and cortical subregions we investigated here $[62,63]$. This thalamic region may play an important supplemental role in the visual representation of context [64]. However, its effect on the $\mathrm{CPu}$ is not yet known. An alternative candidate is the retina-superior colliculus-intralaminar thalamus-CPu pathway [65-67], which converges with tectonigral dopaminergic signals to enhance the detection of unpredictable salient signals [68]. 
Together, the electrical excitability of pVis + neurons from the V2 and the corticostriatal gamma band coherence modulated by the visual percept during self-motion suggest a cortical origin for some aspects of the striatal visual information. However, the lack of electrical excitability of aVis+ neurons also predicts the presence of a complementary subcortical input.

The significance of the visual information processing in the striatum has been shown both in visuomotor associations [69,70] and oculomotor functions [8,71]. The exact role of the various striatal aspects of the visual information in shaping the behavior is less clear because studies perturbing selective visual inputs of the striatum are missing. Striatal responses to nonmoving visual stimuli (mainly flashes and other static stimuli) have been unambiguously described in multiple species $[7,14,72,73]$. However, visual motion is applied mostly in relation to a guided oculomotor or choice task, whose protocols cannot differentiate if $\mathrm{CPu}$ neurons respond to the pure visual features of the motion or the abstract, task-associated meaning of the "cue." The latter explanation is more likely, as responses were reported to enhance over trials of associative learning [74].

Several studies have made attempts to estimate the visual motion-related sensitivity of the striatum $[9,10,29]$. However, none of them have been performed during natural self-motion. Further experiments are needed to understand the role of striatal representations of the passively observed and self-motion-congruent visual motion percepts in neuronal computations and behavior. Speculatively (as projections of the intralaminar thalamic nuclei converge with visual cortical inputs at the striatum), it is possible that one role of this system is to map the external reflections of self-behavior with salient environmental changes during reinforcement learning [73,75]. In addition to the sensory and motor information, the integration of complex representations such as space, memory, reward, and motivations [76] can serve as a high-utility support to a system like that.

\section{Multimodal integration in the dorsomedial striatum-Processing nonsensory modalities}

Despite the remarkable topographic organization of the striatal afferentations, their functionality is not strictly separated by sharp borders but rather form overlapping gradients [77]. The dorsal part is considered to be an associative zone serving multisensory integration [45]. This segment of the $\mathrm{CPu}$ receives a broad repertoire of sensory modalities [7], contextual cues [33], and kinetic and positional information [78], as well as other sources reflecting the internal dynamics of the brain $[7,79]$.

As the animals were running in the linear track, we found a substantial number of neurons possessing place field-like activity [32-34,80,81]. A few of them showed theta phase precession as well [82]. Despite their singular "place fields," the recorded CPu neurons were relatively weak space coders. Their selectivity and sparsity were approximately half and twice as large, respectively, as what we measured earlier in the case of CA1 place cells [35]. These features may be a projection of the hippocampal activity and not a purely de novo-generated feature, since in an earlier study, they have been found to be more strongly coupled to the hippocampal theta phases than to striatal theta [82]. Nonetheless, besides its possible volume-conducted component, striatal theta is also modulated, for example, by reward signals to some extent [82]. In the case of many neurons, the experimental approach of the current study could not reliably differentiate if the recorded spatial firing pattern was purely related to a coding of the location or if it was also shaped by a reward prediction as the animal was approaching the water ports [83]. The place fields were conserved in both running directions in the maze. Thus, the head direction activity of the striatum [80] might not play a key role in forming 
these place fields, although we did not investigate the head direction—related patterns in this study.

Besides the spatial information, striatal neurons are also capable of coding various behaviorally relevant environmental contexts $[4,40]$. To avoid any associations of valence or other behavioral meaning to the "stripedness" of the maze, we designed our aVis stimulation experiment to employ a model-free reinforcement-learning paradigm [84]. The activity of the aVis+ neurons was only partially modulated by the visual stimuli in a similar way to rate remapping $[85,86]$ (i.e., the presence of the striped pattern did not alter the spatial location of the place fields but could modulate the intraplace field FRs [36,37]). This rate modulation was stronger at high temporal frequencies of the perceived visual motion (i.e., at faster running speeds), similarly to the spatiotemporal tuning of the $\mathrm{pVis}+$ neurons in the $\mathrm{pVis}$ experiment. Moreover, we found no difference in the FRs of the aVis+ neurons while the animals were not running and were observing the same but steady patterns. The identical running velocities and trajectories in the two conditions ruled out further that the FR difference between the two conditions was induced by the different motor (i.e., locomotion) patterns. Our additional argument against the contextual but not visual motion modulation of the aVis neurons in the linear maze is that the appearance of the motion of identical striped patterns in the pVis environment did not alter the activity of the aVis+ neurons. These observations suggest that the animals did not consider the two conditions as two independent contexts and highlight the integrative nature of the neuronal activity in the striatum.

Traditionally, the hippocampus and the striatum were considered as two independent but complementary memory systems, respectively responsible for episodic and procedural memory [87]. An alternative concept is that the $\mathrm{CPu}$ should be better considered as an integrator that has the chance to adjust procedural motor functions based on the internal representations of "past experiences" (i.e., through hippocampal episodic and spatial memory [5]) and the "present circumstances" (i.e., through the contribution of sensory systems). Our finding regarding the coexistence and simultaneous, conjunctive expression of sensory-related and contextual/spatial (e.g., hippocampal) features in the CPu may support this latter concept.

\section{Network oscillations in the dorsomedial striatum}

During their striatal integration, the proper timing of the converging inputs of various modalities is orchestrated by local and global oscillations. The origin and significance of the various LFP oscillations in the $\mathrm{CPu}$ are unclear due to the lack of any ordered geometrical alignment of the striatal neurons [88-90]. LFP oscillations can be (1) locally generated by the local reverberating excitatory-inhibitory connections, (2) passively and instantaneously volume-conducted to the site of observation, or (3) evoked locally, driven by rhythmic inputs from distal structures when the local neuronal activity resembles the original oscillation with a synaptic delay. In this third case, the current source density analysis of structures with unordered neurons (e.g., the striatum) will not reveal any characteristic source and sink patterns. Still, as most extracellular recordings in intact animals are referenced to a distal "electroneutral" location (e.g., the cerebellum), even the randomly arranged striatal neurons can give rise to largeamplitude LFP oscillations. This is because they rhythmically sink and release ionic charges from the extracellular space during their synchronized discharges $[91,92]$. In the striatum, all three ways most likely contribute to the generation of LFP oscillations.

Although theta phase coupling is present in a good number of striatal neurons, in vitro studies have not revealed any cellular properties that may support the local generation of the theta rhythms [93]. Since phase precession is more constrained to hippocampal theta than to the local rhythm [82], it is likely that the CPu only resembles the theta-modulated activity 
from the hippocampus or connected structures (e.g., prefrontal cortex [94,95]). Similarly, delta oscillations are imposed by corticostriatal neurons driving striatal interneurons and medium spiny neurons $[44,96]$. However, beta band oscillation can be generated by the $\mathrm{CPu}$ on its own through the interplay between PV+ fast-spiking interneurons and medium spiny neurons under the control of dopamine neuromodulation [97].

The picture is not so clear in the case of gamma oscillations. Van der Meer and colleagues [77] found that at least part of the ventral striatal gamma activity is locally generated. However, the $\mathrm{CPu}$ network lacks the necessary inhibitory-excitatory loop that could generate these rhythms alone $[98,99]$. Along these lines, it is likely that rhythmicity is generated elsewhere (e.g., the hippocampal formation, the piriform, or the visual cortex) and that the striatal neurons only resonate with these inputs $[88,89]$. However, the synchronized, sudden switches between 50 and $80 \mathrm{~Hz}$ gamma rhythms along the whole extent of the ventral striatum also suggest the presence of some coherent internal mechanisms [83].

The distal and local generation of the respective lower- and higher-frequency oscillations is reinforced by the observation that the activation of TFNs selectively increases the power of both the beta and gamma bands but not the lower-frequency bands [97]. We strongly hypothesize that the LFP oscillations of the dorsal striatum are at least partially generated by the coordinated discharges of the striatal neurons that are governed by their distal inputs (including the visual cortex). This idea is supported by the lagging gamma band coherence of the striatal and V2 LFP oscillations, as well as the differential phase locking of the recorded neurons to local and distal gamma oscillations.

\section{The possible role of the corticostriatal LFP oscillations}

We found that the neurons of V2 express oscillations in the low- and mid-gamma band. Such patterns also appear in the $\mathrm{CPu}$ with approximately $20-30$ ms delay (called gamma-50 by van der Meer and Redish [83]). We interpret this delay as a sign of information transfer between the visual cortex and the striatum. This delayed synchrony is not present at other frequency bands (except for the theta band), and it quickly decays, even if the signals are further shifted with an integer number of cycles. This suggests that the detected events are discrete packages of a few gamma cycles but not a persistent gamma oscillation (see S8 Fig for interpretation). The lag of these gamma epochs is in agreement with the latency of the striatal excitatory postsynaptic potentials (EPSPs) compared to the V1-evoked potentials after visual stimulation, as reported by Reig and Silberberg [7]. We assume that these striatal gamma events are likely to be generated by the evoked gamma frequency bursts of PV+ fast-spiking interneurons [100], since similar PV+ interneurons are responsible for gamma oscillations in the hippocampus and cortex [101]. This hypothesis is compatible with our finding that the majority of pVis+ neurons are FFNs, underpinning that their modulation through sensory inputs may have an effect on the generated gamma events. We found that visual stimulation decreases the FR of the FFNs and presumably their cortical afferents as well. Thus, we assume that this decreased cortical "constrain" is the cause of the weaker gamma coherence in the striped maze.

Coherent LFP oscillations serve as "semaphores" for interactions between many circuits of the brain and are efficient filters for information transfer [102]. The detected oscillatory delay of the mid-gamma band matches the delay of the monosynaptic responses recorded in our electrical stimulation experiment. It is therefore possible that its purpose is to offer highly excitable time slots when the $\mathrm{CPu}$ neurons become more sensitive to cortical information. It is doubtful whether these gamma patterns are related only to the visual cortical interactions or that multiple inputs are converging to the $\mathrm{CPu}$ during these short temporal windows, given that the importance of the striatal gamma patterns has already been shown in signal response- 
association tasks [103]. On the other hand, the weak theta phase coupling of the pVis+ neurons reflects their relatively steady responsivity. This is a prerequisite for the reliable quick recognition of moving objects at any moment. Further experiments are needed to gain evidence to verify these hypotheses.

Differential innervation conclusively distinguishes $p V i s+$ cells from the larger pool of perceptors detecting visual dislocations induced by self-motion (i.e., aVis+ neurons) and establishes them as a separate functional group. We propose that the aVis+ neurons may provide robust feedback on the dislocation of the gait caused by the movements of the animal. In addition, successive comparison of the activity of aVis+ and pVis+ neurons can extract the presence of independently moving third-party objects such as prey or predators in a nonstationary (but otherwise neutral) visual environment. This process may be important in detecting selfindependent moving objects and for selecting the appropriate action to challenge them. Moreover, the aVis+ and pVis+ neurons also integrate spatial (and possibly contextual and kinetic) modalities. They are modulated by visual motion co-occurring with self-motion whose features may have a utility in sensorimotor integration while performing the chosen action. Further experiments may confirm these hypotheses by revealing the origin of the visual information that drives the aVis+ neurons and testing the behavioral consequence of the selective manipulation of these two pathways. Optogenetic silencing of the specific axon terminals in the dorsomedial $\mathrm{CPu}$ may be a good approach to achieve this in the future.

\section{Materials and methods}

\section{Ethics statement}

All experiments were approved by the Ethical Committee for Animal Research at the Albert Szent-Györgyi Medical and Pharmaceutical Center of the University of Szeged (No. XIV/218/ 2016 and XIV/471/2012). Thirty-two male rats (Long-Evans, 3-12 mo old) were used in this study.

\section{Awake electrophysiological experiments}

Surgery and recordings. Electrode fabrication and implantation surgery were described in details earlier [26,104]. Briefly, animals were anesthetized with isoflurane, and 1 or several craniotomies were performed under stereotaxic guidance. Sixty-four channel silicon probes (NeuroNexus, Ann-Arbor) were implanted in the dorsomedial striatum. In 2 animals, an additional 32 channel silicon probe was implanted in the visual cortex of the same hemisphere. In 2 other animals, a bipolar stimulation electrode made of two $50 \mu \mathrm{m}$ thick parylene-insulated tungsten wires was implanted in the visual cortex (AP $4.5 \mathrm{~mm}$ posterior from the bregma; ML $2.8 \mathrm{~mm}$; DV 0.5 and $2 \mathrm{~mm}, 1,500 \mu \mathrm{m}$ distance between the tips of the wires, $500 \mu \mathrm{m}$ uninsulated part at each tip). Silicon probes were mounted on custom-made micro-drives to allow their precise vertical movement after implantation. The probes were inserted above the target region, and the micro-drives were attached to the skull with dental cement. The craniotomies were sealed with sterile silicone. Two stainless steel screws were drilled bilaterally over the cerebellum, serving as ground and reference for the recordings. Several additional screws were driven into the skull and covered with dental cement to strengthen the implant. Finally, a copper mesh was attached to the skull with dental cement and connected to the ground screw to act as a Faraday cage, preventing the contamination of the recordings by environmental electric noise. After postsurgery recovery, the probes were moved gradually in 75 to $150 \mu \mathrm{m}$ steps per day until the desired position was reached [104]. The $\mathrm{CPu}$ was identified physiologically by increased unit activity. The exact electrode locations were identified post hoc from histological sections. 
Data acquisition. The operated animals were housed in individual cages and recorded during different navigational tasks. One animal was also recorded during sleep in his home cage. Neuronal recordings were performed daily by connecting the probes to a signal multiplexing headstage attached to a thin and light cable pending from the room ceiling on a trolley system that allowed free movement of the animal. The spatial position of the rats during behavioral sessions was monitored using video tracking of 2 LEDs fixed to the headstage at 30 frames per second, with approximately $3 \mathrm{~mm}$ resolution. The wide-band signal $(0.2-10 \mathrm{kHz})$ acquired at 20K Sample/s (KJE-1001, Amplipex Ltd, Hungary) was low-pass filtered and downsampled to $1,250 \mathrm{~Hz}$ for LFP analysis and high-pass filtered $(>0.5 \mathrm{kHz})$ for spike detection.

Electrical and passive (non-self-motion-induced or self-independent) visual stimulations. A home cage-like visual stimulating environment was created to test the neuronal responses to visual stimuli while the animal was performing nonguided behavior. A $60 \times 60$ $\mathrm{cm}$ rectangular wooden platform was surrounded by four 24" LED monitors (24M45H-B, LG, Seoul, South Korea) serving as approximately $30 \mathrm{~cm}$ tall walls, and the rats were placed in this stimulation box. One stimulus trial consisted of an initial $1 \mathrm{~s}$ uniform gray screen, $1 \mathrm{~s}$ of fullscreen stationary grating with sinusoidally modulated luminance in a given orientation (8 orientations in $45^{\circ}$ steps) and with a given spatial density (170, 85 , or $42.5 \mathrm{~mm} /$ cycle), and finally $1 \mathrm{~s}$ of moving grating, during which the aforementioned grating was sliding orthogonal to its orientation with a given temporal velocity $(1,200,600$, or $300 \mathrm{~mm} / \mathrm{s})$. The overall brightness of the three conditions was adjusted to be isoluminant (i.e., gray screen versus black-to-white-toblack gratings). The spatial densities and temporal velocities of the gratings were chosen to match the spectral preference of the caudate neurons reported earlier, when viewed from the center of the cage, and were combined in a way that gave a constant sliding speed of approximately 7 cycles/s [9]. The frame rate of the monitors was set to $60 \mathrm{~Hz}$; thus, the grating was sled with approximately one-ninth of a cycle in each consecutive frame, which gave the percept of a smooth motion. A pseudorandom sequence of visual stimulus trials was generated by random combinations of direction/orientation (8) and spatial/temporal properties (3), interleaved with $3 \mathrm{~s}$ long trials of blinking full-screen light stimulation at $0.5 \mathrm{~Hz}$ (i.e., a full-screen $1 \mathrm{~s}$ black-1 s white- $1 \mathrm{~s}$ black sequence). The final sequence of two thousand five hundred $3 \mathrm{~s}$ long trials was played on all 4 monitors simultaneously. Each parameter combination was repeated 100 times. The displayed frames were always identical on the 4 monitors, and the room was otherwise completely dark. The visual stimulation was synchronized with the neuronal data by simultaneously recording a digital code displayed at the top-left corner of the screens by a photodetector. This $1 \times 1 \mathrm{~cm}$ large visual code was not visible to the animals, as these parts of the monitors were covered. Animals' behavior was monitored using a web camera, and trials in which they were sleeping instead of random exploration or quite wakefulness were excluded. The same stimulation sequence was used for the initial screening of intrastriatal cFos distribution. To test the sensitivity of the neurons to luminance change, only the trials of the fullscreen flash patterns were analyzed. All stimulation protocol was performed during the dark cycle of the animals.

In animals with stimulating electrodes in the visual cortex, we also tested single-unit and evoked-potential responses in the $\mathrm{CPu}$ for electrical stimulation. Bipolar electrical pulses ( $300 \mu$ s long $500 \mu \mathrm{A}$ pulses repeated every $300 \mathrm{~ms}$ for 5 min during each session) were delivered, and $\mathrm{CPu}$ recordings were performed while the animals were kept in the same stimulating cage as above, with the monitors displaying the uniform gray screens.

Active (self-motion-induced) visual stimulation in the linear maze with dynamically updatable visual environment. In order to investigate the responses of $\mathrm{CPu}$ neurons to visual motion induced by the self-motion of the animals, we constructed a linear maze in 
which the visual environment could be randomly and instantaneously swapped between uniform grayness and sinusoidally modulated gratings while the animal was running for water rewards in the maze. The overall brightness of the two conditions was isoluminant. The visual scene was projected onto the walls and floor of the maze using a video projector (MW663, BenQ, Taipei, Taiwan) in an otherwise dark room. To prevent the animals from casting a shadow and blocking the projection on parts of the environment, which is a bottleneck of projecting from above, the maze was constructed of translucent acrylic glass, and the scene was projected on these walls from outside, using a dual mirror system (see Fig 4A). The rats were trained to run back and forth in this maze to collect small water rewards. These droplets were automatically delivered at water ports located at the two ends of the maze when the animal passed through the infrared gates at the opposite side. These gates were located $10 \mathrm{~cm}$ away from the water ports. After each run, the projected scene was randomly swapped between a uniform gray image and a stationary grating. To match the preferred spatial density of the passive stimulation, 16 grating cycles were projected along the full length $(230 \mathrm{~cm})$ of the maze. As the rats were running at about $85 \mathrm{~cm} / \mathrm{s}$ speed, the temporal velocity of the apparent visual motion (approximately 6 cycles/s) was comparable to that used in the passive stimulation environment.

Postmortem verification of the electrode locations. Following the termination of the experiments, animals were deeply anesthetized. All animals were transcardially perfused first with $0.9 \%$ saline solution followed by $4 \%$ paraformaldehyde (PFA) solution. Brains were sectioned in $60 \mu \mathrm{m}$ thick slices (VT1000S Vibratome, Leica, Wetzlar, Germany) in the coronal plane and stained using 4',6-Diamidino-2-phenylindole dihydrochloride (DAPI; D8417, Sigma-Aldrich). Locations of the electrode tracks were identified using epifluorescent microscopy (AxioImager, Carl Zeiss, Oberkochen, Germany).

\section{Anatomical tract-tracing experiments}

Long-Evans rats (260-580 g) of both sexes were anesthetized with $1 \%-3 \%$ isoflurane and then mounted on a stereotaxic apparatus. Atropine $(0.1 \mathrm{mg} / \mathrm{kg}$, s.c. $)$ was administered immediately after the anesthesia induction. Stages of anesthesia were maintained by confirming the lack of vibrissal movements and nociceptive reflex. The rectal temperature was maintained at 35$37^{\circ} \mathrm{C}$ with a DC temperature controller (TMP-5b; Supertech, Pécs, Hungary). A small incision on the skin was then made for craniotomy after s.c. lidocaine injections.

Anterograde tract tracing of corticostriatal neurons. A craniotomy above the right V2 was made $4.0-5.0 \mathrm{~mm}$ posterior from the bregma, $2.0-3.0 \mathrm{~mm}$ lateral of the midline [105]. A glass capillary (tip 10-20 $\mu \mathrm{m}$ ) filled with 10\% BDA (D1956; Thermo Fisher Scientific, Waltham, MA) in $0.1 \mathrm{M}$ phosphate-buffered saline (PBS) was installed with an auto-nanoliter injector (Nanoject II; Drummond Scientific, Broomall, PA) and then inserted into the V2 according to the following 4 stereotaxic coordinates: (in mm) AP -4.50, ML 2.25; AP -4.50, ML 2.75; AP -5.00, ML 2.25; AP -5.5, ML 2.25. For each coordinate, $0.3 \mu \mathrm{l}$ of the BDA solution was ejected at $0.7,0.85$, and $1 \mathrm{~mm}$ below the pia at a rate of $0.09 \mathrm{nl} / \mathrm{s}$. After ejections, the capillary tip was held in the position for at least $5 \mathrm{~min}$ and then gently retracted. The craniotomy was covered with sterile bone wax, and the skin wound was sutured. After $1 \mathrm{wk}$ of survival, the rats were deeply anesthetized and perfused, and the brains were processed (see section Tissue processing and immunohistochemistry).

Retrograde labeling of striatal afferents. Four Long-Evans rats were anesthetized and then mounted on a stereotaxic apparatus as described in section Anatomical tract-tracing experiments. A craniotomy above the striatum $(\mathrm{CPu})$ was made. A glass capillary filled with 
4\% fluorogold (Fluoro-Gold; Fluorochrome, Denver, $\mathrm{CO}$ ) in $0.9 \%$ saline was installed with an auto-nanoliter injector (Nanoject II) and then inserted into the $\mathrm{CPu}$ according to the following coordinates: (in mm) AP -1.25-1.75, ML 3.6-4.0, and DV 3-4 from the bregma, midline, and pia, respectively. For each coordinate, $0.15-0.30 \mu \mathrm{l}$ of the solution was gently ejected. After $1 \mathrm{wk}$ of survival, the rats were transcardially perfused and fixed; $60 \mu \mathrm{m}$ thick coronal brain sections were prepared and processed as described in section Tissue processing and immunohistochemistry.

\section{Tracing experiments in combination with visual stimulation}

Nine rats underwent the BDA injection of the anterograde tracing as described in section Anatomical tract-tracing experiments. After 1 wk of survival, the rats were reared in the cage for $\mathrm{pVis}$ stimulation (see above) in complete darkness overnight, and then 5 of them were stimulated by the same $\mathrm{pVis}$ stimulation used in the freely moving electrophysiological experiments. The 4 other animals were left in the darkness for the same duration (see Fig 2A). After that, the rats were immediately anesthetized and perfused, and their brains were processed for $\mathrm{cFos}$ and BDA immunohistochemistry as described in section Tissue processing and immunohistochemistry. In 1 animal, we also tested the identity of cFos+ cells with immunohistochemistry against PV.

\section{Tissue processing and immunohistochemistry}

Following the termination of the experiments, animals were deeply anesthetized. All animals were transcardially perfused first with $0.9 \%$ saline solution followed by $4 \%$ PFA solution. Brains were sectioned in 50-70 $\mu \mathrm{m}$ thick coronal slices (VT1000S Vibratome). The following staining procedures were performed at room temperature.

Immunohistochemistry for retrograde tracing. Sections were incubated successively with 10\% NGS in PBS containing 0.3\% Triton X-100 (PBS-X) for $30 \mathrm{~min}, 1: 2,000$ diluted antifluorogold polyclonal antibody (AB153-I; EMD Millipore, Billerica MA) in PBS-XG for $48 \mathrm{~h}$, and $4 \mu \mathrm{g} / \mathrm{ml}$ Alexa Fluor 633-conjugated goat anti-rabbit IgG (A-21071; Thermo Fisher Scientific) in PBS-XG for $2 \mathrm{~h}$. Sections were counterstained with a fluoro-Nissl solution for $60 \mathrm{~min}$, washed with PBS, mounted, and coverslipped.

Immunohistochemistry for the anterograde tracing experiments combined with visual stimulation. Animals were transcardially perfused with physiological saline followed by $4 \%$ $\mathrm{PFA}$ and $0.2 \%$ picric acid in $0.1 \mathrm{M}$ phosphate buffer (PB; $\mathrm{pH}$ 7.2-7.3). After removal, brains were postfixed overnight, embedded in $4 \%$ agarose, coronally sectioned at $50 \mu \mathrm{m}$ thick using a vibrating blade microtome, and harvested in PBS. All incubations were followed by washing with PBS-X. Sections were incubated successively with $10 \%$ normal goat serum (NGS) in PBS-X for $30 \mathrm{~min}, 1 \mu \mathrm{g} / \mathrm{ml}$ rabbit anti-cFos polyclonal antibody (ABE457; EMD Millipore; Kim et al., 2014) in PBS-X containing $1 \%$ NGS and $0.02 \%$ sodium azide (PBS-XG) overnight, and a mixture solution containing $4 \mu \mathrm{g} / \mathrm{ml}$ Alexa Fluor 488-conjugated goat anti-rabbit IgG (A-11034; Thermo Fisher Scientific) and $2 \mu \mathrm{g} / \mathrm{ml}$ Alexa Fluor 555-conjugated streptavidin (S21381; Thermo Fisher Scientific) in PBS-XG for $2 \mathrm{~h}$. Sections were then mounted on glass slides, counterstained with a fluoro-Nissl solution (N-21479; Thermo Fisher Scientific) for 40 min, washed with PBS, and finally coverslipped with $50 \%$ glycerol and $2.5 \%$ triethylene diamine in PBS [106].

cFos and PV double immunohistochemistry. Sections were incubated successively with $3 \%$ bovine serum albumin (BSA) in PBS-X for $30 \mathrm{~min}$, a mixture of first antibodies (rabbit anti-cFos at 1:000: ABE457; EMD Millipore and mouse anti-PV at 1:3,000: PV235; Swant, Marly, Switzerland) in PBS-X containing 1\% BSA (PBS-XBSA) overnight, and a mixture 
solution containing $4 \mu \mathrm{g} / \mathrm{ml}$ Alexa Fluor 488 -conjugated goat anti-rabbit IgG and $4 \mu \mathrm{g} / \mathrm{ml}$ Alexa Fluor 594-donkey anti-mouse IgG (A-21203; Thermo Fisher Scientific) in PBS-XBSA for $2 \mathrm{~h}$. Sections were then mounted, counterstained, and coverslipped.

Confocal microscopy. Images of fluorescently stained sections were captured using a laser scanning confocal microscopy (LSM880; Carl Zeiss). Tile images of $5 \times 5$ and $10 \times 10$ for the visual cortex and the striatum were acquired, respectively. Each tile of $10 \mu \mathrm{m}$ optical thicknesses was taken using a Plan-Apochromat 20×/0.8 M27 objective lens (Carl Zeiss), $1.03 \mu$ s pixel time, and 4 times frame average at $512 \times 512$ resolution. The power of the lasers was $0.4-$ $0.7 \mathrm{~mW}$, which did not induce obvious fading.

\section{Data analysis}

Statistics and data availability. All data analyses were performed in MATLAB (Mathworks, Natick, MA), unless otherwise noted. No specific analysis to estimate minimal population sample was used, but the number of animals, trials, and recorded cells was larger or similar to those employed in previous works, e.g., $[4,83,107]$. No randomization or blinding was employed. For statistical testing, Student $t$ test, Wilcoxon rank-sum test, or KolmogorovSmirnov tests were used, depending on the distribution of the data and the sample size (twotailed). To compensate the cumulative error due to multiple comparisons, Bonferroni correction was employed. One, two, or three asterisks on the figures denote significance levels $<0.05$, $<0.01$, and $<0.005$, respectively. MIs were always calculated as the ratio of the difference and the sum of two measures. The data that support the findings of this study are available from the corresponding author upon reasonable request, and the numerical values used to generate figure plots can be found in S1 Data.

\section{Automated processing of the microscopic images}

Separate color channels of the raw data were exported as 8-bit tiff files. For cFos and PV double immunostaining, cFos-positive, PV-positive, and cFos- and PV-double-positive neurons were manually counted using the Cell counter plugin of ImageJ software (NIH, Bethesda, MD). For the estimation of BDA-labeled axon density and cFos density, the following automated procedure has been employed (see also S1 Fig): For each slice, a binary mask layer was created in Photoshop (Adobe systems, San Jose, $\mathrm{CA}$ ) to void the extra-CPu regions (non-CPu mask) and the obvious artefacts of the BDA channel (intra-CPu mask, e.g. blood vessels). The BDA, the cFos, and the mask channels were saved as separate grayscale image files. The cFos image was preprocessed in ImageJ (v1.50) using the Image-based Tool for Counting Nuclei plugin (ITCN, Kuo and Byun, Center for Bio-image Informatics at UC Santa Barbara, https://imagej. nih.gov/ij/plugins/itcn.html) after image inversion. ITCN (settings: width: $14 \mathrm{px}$, minimum distance $7 \mathrm{px}$, threshold 1, detect dark peaks) marked the cFos+ somata as red pixels on the grayscale image. All three image files were loaded in MATLAB and divided into $512 \times 512$ pixel subregions $(10 \times 10$ pieces $)$. cFos density was measured at the corresponding image as the number of red (somata) pixels in each subregion that were not masked in the mask map, normalized by the nonmasked area. BDA image was thresholded using the mean +4 SD luminosity level of the overall nonmasked image. Pixels brighter than this threshold were considered as BDA-labeled axons. BDA density was calculated the same way as for the cFos density. Intra-CPu masks were covering less than $2 \%$ of the total $\mathrm{CPu}$ area at all slices and were uniformly distributed both along the DV and ML axes and thus did not bias the measured densities. Data of subregions smaller than 2,600 pixels (due to the non-CPu mask) were discarded to avoid numeric approximation errors. ML and DV extent of each CPU slice was normalized when analyzing multiple slices together. 
Spike sorting and unit classification. Neuronal spikes were detected from the digitally high-pass-filtered raw signal $(0.5-5 \mathrm{kHz})$ by a threshold crossing-based algorithm (Spikedetekt2; https://github.com/klusta-team/spikedetekt2). Detected spikes were automatically sorted using the masked EM algorithm for Gaussian mixtures implemented in KlustaKwik2 [24] (https://github.com/klusta-team/klustakwik/), followed by manual adjustment of the clusters using KlustaViewa software [25] (https://github.com/klusta-team/klustaviewa/) to obtain well-isolated single units. Multiunit or noise clusters were discarded for the analysis. Cluster isolation quality was estimated by calculating the isolation distance and interspike interval (ISI) index for each cluster [26]; poor quality clusters were discarded. Striatal neurons were classified as reported by Schmitzer-Torbert and Redish [107], assisted by monosynaptic excitatory and inhibitory interactions between simultaneously recorded, well-isolated units [35]. Briefly, 5 parameters were calculated: spike width and spike trough-to-peak time [26], FR, proportion of interspike intervals exceeding $2 \mathrm{~s}$ (ISI2s), and postspike suppression time (PSST, [107]). Neurons with more than 2\% ISI2s, broad spikes, and low FR and PSST were classified as TFNs; those with less than 2\% ISI2s, narrow spikes, high FR, and low PSST were considered as FFNs; and finally, those with less than 2\% ISI2s, low FR, and high PSST were classified as TFNs. Spike waveforms were extracted $([-1,+1] \mathrm{ms}$ around the threshold crossings) from the raw unfiltered recordings, and their linear trends were removed.

Effect of the $\mathbf{p V i s}$ stimulation on the FRs. Peristimulus time histograms were constructed from the action potential time series, triggered by the visual stimulation sequence. FRs were calculated for the periods during uniformly gray, stationary grating, and moving grating stimulation separately, for each stimulus condition. FR MIs were calculated for each condition for uniform versus stationary and uniform versus moving grating segments as $\mathrm{MI}_{\text {stationer }}=\left(\mathrm{FR}_{\text {stationer }}-\mathrm{FR}_{\text {uniform }}\right) /\left(\mathrm{FR}_{\text {stationer }}+\mathrm{FR}_{\text {uniform }}\right)$, and $\mathrm{MI}_{\text {motion }}=\left(\mathrm{FR}_{\text {moving }}-\right.$ $\left.\mathrm{FR}_{\text {uniform }}\right) /\left(\mathrm{FR}_{\text {moving }}+\mathrm{FR}_{\text {uniform }}\right)$. The same approach was used to compare the neuronal activity in dark and brightness during the luminance test. The effect of the blinking light was judged by the trial-by-trial comparison of the FRs during the black and the white periods of the stimulation.

Spiking activity in the linear maze. Coordinates of the detected LEDs were linearized by projecting them onto the axis of the linear maze, smoothed using a 10 frames wide Gaussian kernel, and rounded to $1 \%$ precision. Successful running trials were considered as periods in the recordings when the animals were traversing from $10 \%$ to $90 \%$ of the maze or vice versa. Automated trial extraction was performed by detecting $50 \%$ crossings in the time series of the linearized positions, and each of the crossing detections were tracked backward and forward in time until the velocity dropped below $5 \mathrm{~cm} / \mathrm{s}$. Trials in which either of these automatically detected start and stop positions were between $10 \%$ and $90 \%$ (i.e., the rat stopped in the middle of the maze) were discarded. Running directions were arbitrarily named "forward" and "backward" runs. Spatially resolved FR histograms and raster plots were compensated by the occupancy frequency of each spatial bin (20 bins) [30]. To determine the responsivity of the neurons in the aVis task, the mean compensated FRs within these spatial bins were compared between the uniform and striped trials using a paired $t$ test. MIs were calculated to express the strength of the overall effect of perceived visual motion by comparing the FRs during "striped" and "uniform" trials as $\mathrm{MI}=\left(\mathrm{FR}_{\text {striped }}-\mathrm{FR}_{\text {uniform }}\right) /\left(\mathrm{FR}_{\text {striped }}+\mathrm{FR}_{\text {uniform }}\right)$.

The spatial extent of the significant modulation of the neuronal FRs by the visual motion was tested by a label-shuffling approach described in [30]. Briefly, the uniform/striped labels of the trials in each session were shuffled, and the difference of spatially resolved firing patterns during the shuffled "uniform" and the shuffled "striped" trials were calculated. This was repeated 1,000 times to create a surrogate dataset of shuffled differences. Local confidence boundaries of the shuffled datasets were determined at the $2.5 \%-2.5 \%$ tails of the distributions 
of the shuffled difference values at each spatial bin ("local significance levels"). To compensate the cumulative error of multiple comparison, a "global significance level" was subsequently determined in an iterative way by increasing the local significance levels until only $5 \%$ of the shuffled surrogate dataset broke the global threshold. This global significance level was used then to determine the significance of difference of the real, unshuffled firing patterns, smoothed by a 5 bin wide (around $15 \mathrm{~cm}$ ) moving average.

To correlate the modulation of the FRs with the temporal frequency of the perceived visual motion (i.e., the running speed), the instantaneous running speeds were binned and averaged in 10-cm-wide spatial bins, and the median of the corresponding MIs of the simultaneously recorded aVis+ neurons were calculated in each bin. Sessions with fewer than $3 \mathrm{aVis}+$ neurons and bins with nonsense MIs (NaNs and Infs due to undersampling errors) were excluded. Because of the low number of spikes in the spatial bins, we calculated the median of the binwise MIs of the aVis+ cells within each trial. Extreme (nonsense) median MIs were further excluded by taking the median of the binned MIs with similar corresponding velocities across trials of a given session. These cleaned MIs were correlated then with the corresponding instantaneous running speeds.

Space-resolved neuronal activity patterns were also analyzed with respect to the local luminance at the instantaneous positions of the rats: each location was assigned with the local luminance value of the "striped" pattern (expressed as the phase of the sinusoidally modulated luminance grating). Circular histogram of the "stripe phase"-resolved activity pattern was generated by averaging the activity patterns across all 14 cycles and all trials of the given condition. For the "uniform" condition with nonmodulated gray pattern, the same cycle phase values were used in the analysis as determined in the "striped" condition. Activity patterns were tested for circular uniformity using the Rayleigh test, and only those neurons were included in the population analysis that showed a significant deviation from the von Mises distribution either in the "striped" or in the "uniform" condition. This deviation was quantified as the MI of circular nonuniformity $\left(\mathrm{MI}=\left[\mathrm{FR}_{\text {peak }}-\mathrm{FR}_{\text {peak-180 }}\right] /\left[\mathrm{FR}_{\text {peak }}+\mathrm{FR}_{\text {peak-180 }}{ }^{\circ}\right]\right)$.

LFP spectral analysis and spike-LFP coupling. Power spectrum and time-resolved spectral analysis were performed on the low-pass-filtered and downsampled (1,250 S/s) LFP signals using multitaper spectral analysis. Spectra were whitened by multiplying with the corresponding frequencies and expressed as decibels (dB). For all local spike-LFP coupling analyses, the median of the local LFP signals recorded on the same shank has been used. For phase coupling of the CPu neurons to V2 LFP, the median LFP of all V2 recording sites was used instead. Subjectively noisy LFP channels due to recording sites with too-high impedance were discarded. LFP signals were filtered in 5 frequency bands (delta: $[1-4 \mathrm{~Hz}]$, theta: $[4-12 \mathrm{~Hz}$, beta: [12-30 Hz], low-gamma: [30-45 Hz], mid-gamma: [45-80 Hz]) with an eighth-order zero-phase-lag Butterworth filter, and phase values were obtained using Hilbert transform. No $50 \mathrm{~Hz}$ notch filtering was used in the data acquisition or during the data analysis. LFP phase histograms were constructed by taking the corresponding instantaneous LFP phase values corresponding to the isolated single-unit spikes, and their deviation from the circular uniform distribution was tested using the Rayleigh test. The bin size of the histograms was $18^{\circ}$ and was smoothed by calculating a 5 bin wide moving average of the histograms. The phase values corresponding to the peaks and troughs of the smoothed distributions were taken as preferred and unpreferred phases, respectively. Phase MIs were calculated from smoothed occurrence frequencies of spikes at the preferred and unpreferred phases to account for possible asymmetric distributions $\left(\mathrm{MI}=\left[\mathrm{FR}_{\max }-\mathrm{FR}_{\min }\right] /\left[\mathrm{FR}_{\max }+\mathrm{FR}_{\min }\right]\right)$.

Spatial location-related activity in the linear maze. Rate map, spatial information, selectivity, and sparsity [35] were calculated for each direction separately. A Gaussian kernel $(\mathrm{SD}=5 \mathrm{~cm})$ was applied to both raw maps of spike and occupancy, and a smoothed rate map 
was constructed by dividing the smoothed spike map by the smoothed occupancy map. A place field was defined as a continuous region of at least $15 \mathrm{~cm}$ where the $\mathrm{FR}$ was above $10 \%$ of the peak rate in the maze, the peak FR was $>2 \mathrm{~Hz}$, and the spatial coherence was $>0.7$. Place fields with fewer than 50 spikes, those that included the turning position of the track, and truncated or overlapping fields were discarded $[35,104]$. Place fields in both running directions in the linear track were treated independently. Stability of place fields across conditions was tested by calculating the spatial correlation of the binned mean firing patterns in the striped and uniform trials.

Cross-region LFP activity analysis. Median LFP signals were calculated for each recording shank (8 recording sites per shank, $8 \mathrm{CPu}$ shanks, and 4 cortical shanks). Granger causality was estimated for each V2 and $\mathrm{CPu}$ signal pairs. Only time lags in the range of $[2,40] \mathrm{ms}$ were considered, and the lag with the highest causality measure was displayed. F-statistics were compared with paired $t$ test between the uniform and striped conditions. Time and frequencyresolved cross-correlations were calculated for each shank pairs (32 in total) the following way: Both signals were iteratively filtered between 1 and $100 \mathrm{~Hz}$ in $1 \mathrm{~Hz}$ steps, with an eighth-order, zero-phase-lag passband filters, and their cross-correlations were calculated for $[-100,+100]$ $\mathrm{ms}$ lags, in $2 \mathrm{~ms}$ steps. The magnitudes of the cross-correlograms were taken as the absolute values of its Hilbert transforms for each frequency. To remove the additive distortion caused by the symmetric correlation of the synchronous events (e.g., common noise), the minima of each symmetric time bin pairs have been subtracted from the same bins. The principle of this approach is demonstrated by analyzing 2 artificial LFP signals and explained on S8 Fig.

\section{Supporting information}

S1 Data.

(XLSX)

S1 Fig. Steps of the automated detection of cFos+ cell and BDA-labeled axon density and cortical cFos expression. (A) Original tile-scan image of the BDA-labeled axons on a representative example slice (left). Middle panel shows the intensity-thresholded image, while right panel denotes the calculated binned axon density. (B) Original tile-scan of the cFos+ neuronal somata on the same slice (left) and its inverted version (middle). Inset of the middle panel shows a magnified image of a cFos+ neuron. Note the red dot placed at the center of the soma by the automated detection algorithm. Right panel shows the binned density of the automatically detected cFos+ neurons. (C) Manually generated image mask to avoid artefact detection. Black mask covers the non-CPu structures, while red masks cover the intra- $\mathrm{CPu}$ artefacts (e.g., noise, vessels, etc.). (D) Distribution of intra-CPU masks at all slices along the vertical (blue) and mediolateral (red) axes. Note that the masks were distributed uniformly and covered only approximately $1 \%$ of the total $\mathrm{CPu}$ area. (E) Correlation of the binned BDA and cFos density values of the representative slice shown on panels $\mathrm{A}-\mathrm{C}$. The correlation coefficient of the value pairs and the significance level of the correlation are displayed above the plot. (F,G) Small (left) and large (right) magnification photomicrographs of the V2 injection site of the BDA tracer (F) and the V1 (G) of a stimulated animal. Color channels are identical to those on Fig 2. Note the large number of cFos+ neurons in both regions as a result of the visual stimulation. $\mathrm{BDA}$, biotinylated dextran amine; $\mathrm{CPu}$, caudate putamen; $\mathrm{V} 1$, primary visual cortex; $\mathrm{V} 2$, secondary visual cortex.

(TIF)

S2 Fig. Basic electrophysiological properties and intrastriatal connectivity of the recorded CPu neurons. $(A-C)$ Waveform and spike train characteristics of a representative PFN (A), 
FFN (B) and TFN (C). Top left panels: mean extracellular spike waveforms on the 8 recording sites of the corresponding electrode shank. Top-right panels: distribution of ISIs, ISI2s are aggregated. Note the high proportion of long ISIs for PFNs (red arrow). Bottom-left and right panels display the postspike suppression histograms and autocorrelograms, respectively. (D) Classification of the recorded neurons based on the features of their spike waveforms (left panel) and spike trains (right panel). (E) Proportion of the recorded PFNs (64\%), FFNs (22\%), TFNs (8\%), and unclassified neurons (6\%) among all recorded single units. $\mathrm{CPu}$, caudate putamen; FFN, fast-firing neuron; ISI, interspike interval; ISI2s, interspike interval exceeding $2 \mathrm{~s}$; PFN, phasically firing neuron; TFN, tonically firing neuron.

(TIF)

S3 Fig. (A-D) Rate remapping of 4 example aVis+ neurons. The left panels of each example show the spatially resolved firing patterns in the uniform and striped environments (blue and red, respectively). Right panels show the details of statistical testing of the space-resolved visual modulation. The thick black lines show the real FR differences in the two environments, while the gray curves denote the label-shuffled surrogate firing pattern differences. Purple and green lines depict the "local" and "global" significance thresholds, respectively. Spatial locations breaking the global significance limits are marked with thick red lines in both the left and right panels. aVis, active visual; $\mathrm{FR}$, firing rate.

(TIF)

S4 Fig. FRs of the pVis+ cells in the "striped" versus "uniform" maze. The plots are showing the comparison of the normalized FRs of each pVis+ neurons in the uniform (abscissa) and in the striped maze (ordinate). Identity and regression lines are shown in black and red, respectively. Correlation coefficients and significance levels are shown above each plot. To form the FR, data points of only those consecutive trials were included in which the condition was changing from "uniform" to "striped" or vice versa. FR values were normalized by using the maximum rate during the "uniform" trials. FR, firing rate; $p V i s$, passive visual.

S5 Fig. Comparison of place preference of the neurons expressing place field-like activity in the "uniform" and the "striped" environment. The plots show the comparison of the normalized FRs of each place cell-like neurons in the uniform (abscissa) and in the striped maze (ordinate). Identity and regression lines are shown in black and red, respectively. Correlation coefficients, significance levels, and the coefficients of the regression lines ("a" and "b," in which $y=a \times x+b$ ) are shown above each plot. To form the FR, data points of only those consecutive trials were included in which the condition was changing from "uniform" to "striped" or vice versa. FR values were normalized by using the maximum rate during the "uniform" trials. FR, firing rate.

S6 Fig. Response profiles of the aVis+ neurons in relation to space, context, velocity, and luminance. (A) Modulation indices of the rate remapping depend on the temporal velocity of the perceived visual motion, determined by the running velocity. Slower speeds elicited weaker decrease (i.e., weaker modulation) of the FRs. (B) Comparison of the mean running speeds in the striped and uniform environments. (C-E) Analysis of aVis+ neurons' activity patterns in the reward zones, where the rats were not running-thus, no visual motion was perceivedbut they still observed the stationary patterns of the walls. (C) The average time spent at the reward zones in trials when uniform or striped pattern were presented was similar. (D) The FRs of the aVis+ neurons were almost identical at the reward zones, with no respect to the pattern or context. (E) The distribution of the significance levels of the cell-by-cell comparison of 
the firing patterns depict that only a small minority of the aVis+ neurons perceived the stationary patterns as different. (F) Two representative neurons excited (left) or inhibited (right) by the global brightness increase. (G) Percent of neurons responsive to global luminance change. Note that in contrast to the aVis+ neurons, more than half of the pVis+ neurons were sensitive to brightness. $(\mathrm{H})$ Response profile of a representative neuron triggered by the individual sinusoidal cycles of the grated pattern. Local luminance levels are shown above the plot; 2 full stripe cycles are shown for better visibility. Note that the stripe phase-resolved firing pattern (red) is similarly uniform as the pattern in the uniform trials (blue). (I) Mean stripe phase-resolved circular modulation indices of the aVis+ neurons were similar in the two conditions. aVis, active visual; $\mathrm{FR}$, firing rate.

(TIF)

S7 Fig. LFP phase coupling of the recorded neurons. Preferred LFP phases are shown as histograms for $\mathrm{pVis-/aVis-} \mathrm{(top} \mathrm{row),} \mathrm{pVis}+$ (second row), and aVis+ (third row) neurons separately. Bottom row shows the comparison of phase coupling strength expressed as the distribution of the phase modulation indices for the three groups of neurons (for quantification, see Fig 5C). Columns from left to right show the phase preferences for band pass-filtered LFP signals in the delta, theta, beta, low-gamma, and mid-gamma bands. For better visibility, the circular distributions are plotted twice $\left(-180-540^{\circ}\right)$ on the phase preference distribution histograms. aVis, active visual; LFP, local field potential; $p$ Vis, passive visual. (TIF)

S8 Fig. Demonstration of the concept of the novel time lag and frequency-resolved crosscorrelation estimation method. (A) A demonstrative example of 2 artificial LFP signals with a phase-delayed continuous theta oscillation, 2 time-delayed discrete gamma bursts in the low- and high-gamma band, and a coincident square wave resembling a recording artefact. (B) Raw time and frequency-resolved cross-correlograms constructed from individual cross-correlograms calculated after iterative filtering for each frequency band. (C) The same time and frequency-resolved cross-correlogram after the suppression of the zero-lag correlation components. Note the emergence of 3 domains (red arrows), representing the phase-shifted theta oscillations and the gamma bursts with the appropriate time lags. Note also the lack of the artefact's spectral profile, which dominated the raw spectrogram on panel B. LFP, local field potential.

\section{Acknowledgments}

We thank Gábor Kozák, Rodrigo Sierra, Lizeth Pedraza, Qun Li, Ferenc Mátyás, Magor L. Lörincz, and Darren Clark for insightful comments, Mari Takeuchi for technical assistance, and Péter Hegyi and József Maléth for providing access to a confocal microscopy.

\section{Author Contributions}

Conceptualization: Anett J. Nagy, Antal Berényi.

Data curation: Anett J. Nagy, Yuichi Takeuchi, Antal Berényi.

Formal analysis: Anett J. Nagy, Yuichi Takeuchi, Antal Berényi.

Funding acquisition: Antal Berényi.

Investigation: Anett J. Nagy, Yuichi Takeuchi, Antal Berényi. 
Methodology: Anett J. Nagy, Yuichi Takeuchi, Antal Berényi.

Project administration: Anett J. Nagy, Antal Berényi.

Resources: Anett J. Nagy, Antal Berényi.

Software: Yuichi Takeuchi, Antal Berényi.

Supervision: Antal Berényi.

Validation: Antal Berényi.

Visualization: Anett J. Nagy, Antal Berényi.

Writing - original draft: Anett J. Nagy, Yuichi Takeuchi, Antal Berényi.

Writing - review \& editing: Anett J. Nagy, Yuichi Takeuchi, Antal Berényi.

\section{References}

1. Nelson AB, Kreitzer AC. Reassessing Models of Basal Ganglia Function and Dysfunction. Annu Rev Neurosci. 2014; 37: 117-135. https://doi.org/10.1146/annurev-neuro-071013-013916 PMID: 25032493

2. Kravitz A V, Tye LD, Kreitzer AC. Distinct roles for direct and indirect pathway striatal neurons in reinforcement. Nat Neurosci. 2012; 15: 816-818. https://doi.org/10.1038/nn.3100 PMID: 22544310

3. Horvitz JC. Stimulus-response and response-outcome learning mechanisms in the striatum. Behav Brain Res. 2009; 199: 129-140. https://doi.org/10.1016/j.bbr.2008.12.014 PMID: 19135093

4. Barnes TD, Kubota Y, Hu D, Jin DZ, Graybiel AM. Activity of striatal neurons reflects dynamic encoding and recoding of procedural memories. Nature. 2005/10/21. 2005; 437: 1158-1161. https://doi.org/ 10.1038/nature04053 PMID: 16237445

5. Akhlaghpour H, Wiskerke J, Choi JY, Taliaferro JP, Au J, Witten IB. Dissociated sequential activity and stimulus encoding in the dorsomedial striatum during spatial working memory. Elife. 2016; 5. https://doi.org/10.7554/eLife.19507 PMID: 27636864

6. Pennartz CMA, Ito R, Verschure PFMJ, Battaglia FP, Robbins TW. The hippocampal-striatal axis in learning, prediction and goal-directed behavior. Trends Neurosci. 2011; 34: 548-59. https://doi.org/10. 1016/j.tins.2011.08.001 PMID: 21889806

7. Reig R, Silberberg G. Multisensory integration in the mouse striatum. Neuron. 2014; 83: 1200-12. https://doi.org/10.1016/j.neuron.2014.07.033 PMID: 25155959

8. Hikosaka O, Sakamoto M, Usui S. Functional properties of monkey caudate neurons. II. Visual and auditory responses. J Neurophysiol. 1989; 61: 799-813. https://doi.org/10.1152/jn.1989.61.4.799 PMID: 2723721

9. Nagy A, Berényi A, Wypych M, Waleszczyk WJ, Benedek G. Spectral receptive field properties of visually active neurons in the caudate nucleus. Neurosci Lett. 2010; 480: 148-153. https://doi.org/10. 1016/j.neulet.2010.06.030 PMID: 20561561

10. Nagypál T, Gombköto P, Barkóczi B, Benedek G, Nagy A. Activity of caudate nucleus neurons in a visual fixation paradigm in behaving cats. PLoS ONE. 2015; 10: 1-18. https://doi.org/10.1371/journal. pone.0142526 PMID: 26544604

11. Yanike M, Ferrera VP. Interpretive monitoring in the caudate nucleus. Elife. 2014; 3: 1-16. https://doi. org/10.7554/eLife.03727 PMID: 25415238

12. Buxbaum-Conradi $\mathrm{H}$, Ewert JP. Responses of single neurons in the toad's caudal ventral striatum to moving visual stimuli and test of their efferent projection by extracellular antidromic stimulation/recording techniques. Brain Behav Evol. 1999; 54: 338-54. https://doi.org/10.1159/000006633 PMID: 10681604

13. Hitier M, Besnard S, Smith PF. Vestibular pathways involved in cognition. Front Integr Neurosci. 2014; 8: 59. https://doi.org/10.3389/fnint.2014.00059 PMID: 25100954

14. Nagy A, Paróczy Z, Norita M, Benedek G. Multisensory responses and receptive field properties of neurons in the substantia nigra and in the caudate nucleus. Eur J Neurosci. 2005; 22: 419-424. https://doi.org/10.1111/j.1460-9568.2005.04211.x PMID: 16045495

15. Crapse TB, Sommer MA. Corollary discharge across the animal kingdom. Nat Rev Neurosci. 2008; 9 : 587-600. https://doi.org/10.1038/nrn2457 PMID: 18641666 
16. Sperry RW. Neural basis of the spontaneous optokinetic response produced by visual inversion. $J$ Comp Physiol Psychol. 1950; 43: 482-9. PMID: 14794830

17. Miall RC, Wolpert DM. Forward Models for Physiological Motor Control. Neural Networks. 1996; 9 : 1265-1279. https://doi.org/10.1016/S0893-6080(96)00035-4 PMID: 12662535

18. Penney JB, Young AB. Striatal inhomogeneities and basal ganglia function. Mov Disord. 1986; 1: 315. https://doi.org/10.1002/mds.870010102 PMID: 2848190

19. Ding L, Gold J. The basal ganglia's contributions to perceptual decision making. Neuron. 2013; 79: 640-649. https://doi.org/10.1016/j.neuron.2013.07.042 PMID: 23972593

20. Khibnik LA, Tritsch NX, Sabatini BL. A direct projection from mouse primary visual cortex to dorsomedial striatum. PLoS ONE. 2014; 9: e104501. https://doi.org/10.1371/journal.pone.0104501 PMID: 25141172

21. Nagy AJ, Berényi A, Gulya K, Norita M, Benedek G, Nagy A. Direct projection from the visual associative cortex to the caudate nucleus in the feline brain. Neurosci Lett. 2011; 503: 52-57. https://doi.org/ 10.1016/j.neulet.2011.08.007 PMID: 21864648

22. Rokszin A, Márkus Z, Braunitzer G, Berényi A, Benedek G, Nagy A. Visual pathways serving motion detection in the mammalian brain. Sensors. 2010; 10: 3218-3242. https://doi.org/10.3390/ s100403218 PMID: 22319295

23. Serizawa M, McHaffie JG, Hoshino K, Norita M. Corticostriatal and corticotectal projections from visual cortical areas 17, 18 and 18a in the pigmented rat. Arch Histol Cytol. 1994; 57: 493-507. PMID 7537509

24. Kadir SN, Goodman DFM, Harris KD. High-Dimensional Cluster Analysis with the Masked EM Algorithm. Neural Comput. 2014; 26: 2379-2394. https://doi.org/10.1162/NECO_a_00661 PMID: 25149694

25. Rossant C, Kadir SN, Goodman DFM, Schulman J, Hunter MLD, Saleem AB, et al. Spike sorting for large, dense electrode arrays. Nat Neurosci. 2016; 19: 634-41. https://doi.org/10.1038/nn.4268 PMID: 26974951

26. Berényi A, Somogyvári Z, Nagy AJ, Roux L, Long JD, Fujisawa S, et al. Large-scale, high-density (up to 512 channels) recording of local circuits in behaving animals. J Neurophysiol. 2014; 111: 1132-49. https://doi.org/10.1152/jn.00785.2013 PMID: 24353300

27. Berke J. Uncoordinated firing rate changes of striatal fast-spiking interneurons during behavioral task performance. J Neurosci. 2008; 28: 10075-80. https://doi.org/10.1523/JNEUROSCI.2192-08.2008 PMID: 18829965

28. Clifford CWG, Ibbotson MR. Fundamental mechanisms of visual motion detection: Models, cells and functions. Prog Neurobiol. 2002; 68: 409-437. https://doi.org/10.1016/S0301-0082(02)00154-5 PMID: 12576294

29. Gombkötő P, Rokszin A, Berényi A, Braunitzer G, Utassy G, Benedek G, et al. Neuronal code of spatial visual information in the caudate nucleus. Neuroscience. 2011; 182: 225-31. https://doi.org/10. 1016/j.neuroscience.2011.02.048 PMID: 21376107

30. Fujisawa S, Amarasingham A, Harrison MT, Buzsáki G. Behavior-dependent short-term assembly dynamics in the medial prefrontal cortex. Nat Neurosci. 2008; 11: 823-33. https://doi.org/10.1038/nn. 2134 PMID: 18516033

31. Hunnicutt BJ, Jongbloets BC, Birdsong WT, Gertz KJ, Zhong H, Mao T. A comprehensive excitatory input map of the striatum reveals novel functional organization. Elife. 2016; 5: e19103. https://doi.org/ 10.7554/eLife.19103 PMID: 27892854

32. Wiener SI. Spatial and behavioral correlates of striatal neurons in rats performing a self-initiated navigation task. J Neurosci. 1993; 13: 3802-3817. PMID: 8366346

33. Berke JD, Breck JT, Eichenbaum H. Striatal Versus Hippocampal Representations During Win-Stay Maze Performance. J Neurophysiol. 2009; 101: 1575-1587. https://doi.org/10.1152/jn.91106.2008 PMID: 19144741

34. O'Keefe J, Dostrovsky J. The hippocampus as a spatial map. Preliminary evidence from unit activity in the freely-moving rat. Brain Res. 1971; 34: 171-5. PMID: 5124915

35. Oliva A, Fernández-Ruiz A, Buzsáki G, Berényi A. Spatial coding and physiological properties of hippocampal neurons in the Cornu Ammonis subregions. Hippocampus. 2016; 26: 1593-1607. https:// doi.org/10.1002/hipo.22659 PMID: 27650887

36. Leutgeb S, Leutgeb JK, Barnes CA, Moser EI, McNaughton BL, Moser M-B. Independent codes for spatial and episodic memory in hippocampal neuronal ensembles. Science. 2005; 309: 619-23. https://doi.org/10.1126/science.1114037 PMID: 16040709

37. Leutgeb JK, Leutgeb S, Moser M-B, Moser El. Pattern separation in the dentate gyrus and CA3 of the hippocampus. Science. 2007; 315: 961-6. https://doi.org/10.1126/science.1135801 PMID: 17303747 
38. Trongnetrpunya A, Nandi B, Kang D, Kocsis B, Schroeder CE, Ding M. Assessing Granger Causality in Electrophysiological Data: Removing the Adverse Effects of Common Signals via Bipolar Derivations. Front Syst Neurosci. 2015; 9: 189. https://doi.org/10.3389/fnsys.2015.00189 PMID: 26834583

39. Sasaki R, Angelaki DE, DeAngelis GC. Dissociation of self-motion and object motion by linear population decoding that approximates marginalization. J Neurosci. 2017; 1177-17. https://doi.org/10.1523/ JNEUROSCI.1177-17.2017 PMID: 29030435

40. Aosaki T, Tsubokawa H, Ishida A, Watanabe K, Graybiel AM, Kimura M. Responses of tonically active neurons in the primate's striatum undergo systematic changes during behavioral sensorimotor conditioning. J Neurosci. 1994; 14: 3969-3984. PMID: 8207500

41. Tepper JM, Tecuapetla F, Koós T, Ibáñez-Sandoval O. Heterogeneity and diversity of striatal GABAergic interneurons. Front Neuroanat. 2010; 4: 150. https://doi.org/10.3389/fnana.2010.00150 PMID: 21228905

42. Ibanez-Sandoval O, Tecuapetla F, Unal B, Shah F, Koos T, Tepper JM. A Novel Functionally Distinct Subtype of Striatal Neuropeptide Y Interneuron. J Neurosci. 2011; 31: 16757-16769. https://doi.org/ 10.1523/JNEUROSCI.2628-11.2011 PMID: 22090502

43. Sharott A, Moll CKE, Engler G, Denker M, Grün S, Engel AK. Different Subtypes of Striatal Neurons Are Selectively Modulated by Cortical Oscillations. J Neurosci. 2009; 29: 4571-4585. https://doi.org/ 10.1523/JNEUROSCI.5097-08.2009 PMID: 19357282

44. Mallet N, Le Moine C, Charpier S, Gonon F. Feedforward inhibition of projection neurons by fast-spiking GABA interneurons in the rat striatum in vivo. J Neurosci. 2005; 25: 3857-69. https://doi.org/10. 1523/JNEUROSCI.5027-04.2005 PMID: 15829638

45. Thorn CA, Graybiel AM. Differential Entrainment and Learning-Related Dynamics of Spike and Local Field Potential Activity in the Sensorimotor and Associative Striatum. J Neurosci. 2014; 34: 28452859. https://doi.org/10.1523/JNEUROSCI.1782-13.2014 PMID: 24553926

46. Berretta S, Parthasarathy HB, Graybiel AM. Local release of GABAergic inhibition in the motor cortex induces immediate-early gene expression in indirect pathway neurons of the striatum. J Neurosci. 1997; 17: 4752-4763. PMID: 9169535

47. Hughes $P$, Dragunow $M$. Induction of immediate-early genes and the control of neurotransmitter-regulated gene expression within the nervous system. Pharmacol Rewiews. 1995;

48. Galletti $C$, Fattori $P$. The dorsal visual stream revisited: Stable circuits or dynamic pathways? Cortex. 2018; 98: 203-217. https://doi.org/10.1016/j.cortex.2017.01.009 PMID: 28196647

49. Zeki S. Area V5-a microcosm of the visual brain. Front Integr Neurosci. 2015; 9: 1-18.

50. Warren PA, Rushton SK. Optic Flow Processing for the Assessment of Object Movement during Ego Movement. Curr Biol. 2009; 19: 1555-1560. https://doi.org/10.1016/j.cub.2009.07.057 PMID: 19699091

51. Palagina G, Meyer JF, Smirnakis SM. Complex Visual Motion Representation in Mouse Area V1. J Neurosci. 2017; 37: 164-183. https://doi.org/10.1523/JNEUROSCI.0997-16.2017 PMID: 28053039

52. Muir DR, Roth MM, Helmchen F, Kampa BM. Model-based analysis of pattern motion processing in mouse primary visual cortex. Front Neural Circuits. 2015; 9: 1-14.

53. Saleem AB, Ayaz A, Jeffery KJ, Harris KD, Carandini M. Integration of visual motion and locomotion in mouse visual cortex. Nat Neurosci. 2013; 16: 1864-1869. https://doi.org/10.1038/nn.3567 PMID: 24185423

54. Furlan M, Smith AT. Global Motion Processing in Human Visual Cortical Areas V2 and V3. J Neurosci. 2016; 36: 7314-7324. https://doi.org/10.1523/JNEUROSCI.0025-16.2016 PMID: 27383603

55. Sereno MI, Allmann JM. Cortical Visual Areas in Mammals. In: Leventhal AG, editor. The Neural Basis of Visual Function. London: Macmillan; 1991. pp. 160-172.

56. Andermann ML, Kerlin AM, Roumis DK, Glickfeld LL, Reid RC. Functional specialization of mouse higher visual cortical areas. Neuron. 2011; 72: 1025-1039. https://doi.org/10.1016/j.neuron.2011.11. 013 PMID: 22196337

57. Saint-Cyr JA, Ungerleider LG, Desimone R. Organization of visual cortical inputs to the striatum and subsequent outputs to the pallido-nigral complex in the monkey. J Comp Neurol. 1990; 298: 129-156. https://doi.org/10.1002/cne.902980202 PMID: 1698830

58. Mikami A, Newsome WT, Wurtz RH. Motion selectivity in macaque visual cortex. I. Mechanisms of direction and speed selectivity in extrastriate area MT. J Neurophysiol. 1986; 55: 1308-1327. https:// doi.org/10.1152/jn.1986.55.6.1308 PMID: 3016210

59. Bair W, Cavanaugh JR, Smith MA, Movshon JA. The Timing of Response Onset and Offset in Macaque Visual Neurons. J Neurosci. 2002; 22: 3189-3205. http://doi.org/20026257 PMID: 11943820 
60. Hammond $\mathrm{P}, \mathrm{Kim} \mathrm{JN}$. Role of suppression in shaping orientation and direction selectivity of complex neurons in cat striate cortex. J Neurophysiol. 1996; 75: 1163-76. Available: http://www.ncbi.nlm.nih. gov/pubmed/8867126 https://doi.org/10.1152/jn.1996.75.3.1163 PMID: 8867126

61. Gizzi MS, Katz E, Schumer RA, Movshon JA. Selectivity for orientation and direction of motion of single neurons in cat striate and extrastriate visual cortex. J Neurophysiol. 1990; 63: 1529-1543. https:// doi.org/10.1152/jn.1990.63.6.1529 PMID: 2358891

62. Rubio-Garrido P, Pérez-De-Manzo F, Porrero C, Galazo MJ, Clascá F. Thalamic input to distal apical dendrites in neocortical layer 1 is massive and highly convergent. Cereb Cortex. 2009; 19: 23802395. https://doi.org/10.1093/cercor/bhn259 PMID: 19188274

63. Kamishina H, Yurcisin GH, Corwin J V., Reep RL. Striatal projections from the rat lateral posterior thalamic nucleus. Brain Res. 2008; 1204: 24-39. https://doi.org/10.1016/j.brainres.2008.01.094 PMID: 18342841

64. Roth MM, Dahmen JC, Muir DR, Imhof F, Martini FJ, Hofer SB. Thalamic nuclei convey diverse contextual information to layer 1 of visual cortex. Nat Neurosci. 2015; 19: 299-307. https://doi.org/10. 1038/nn.4197 PMID: 26691828

65. Coizet V, Overton PG, Redgrave P. Collateralization of the Tectonigral Projection with Other Major Output Pathways of Superior Colliculus in the Rat. J Comp Neurol. 2007; 500: 1034-1049. https://doi. org/10.1002/cne.21202 PMID: 17183537

66. Harting JK, Updyke B V, Van Lieshout DP. The visual-oculomotor striatum of the cat: functional relationship to the superior colliculus. Exp brain Res. 2001; 136: 138-42. PMID: 11204409

67. Schulz JM, Redgrave P, Mehring C, Aertsen A, Clements KM, Wickens JR, et al. Short-Latency Activation of Striatal Spiny Neurons via Subcortical Visual Pathways. J Neurosci. 2009; 29: 6336-6347. https://doi.org/10.1523/JNEUROSCI.4815-08.2009 PMID: 19439610

68. Redgrave P, Prescott TJ, Gurney K. The basal ganglia: a vertebrate solution to the selection problem? Neuroscience. 1999; 89: 1009-23. PMID: 10362291

69. Hoshi E. Cortico-basal ganglia networks subserving goal-directed behavior mediated by conditional visuo-goal association. Front Neural Circuits. 2013; 7: 158. https://doi.org/10.3389/fncir.2013.00158 PMID: 24155692

70. Hadj-Bouziane F, Boussaoud D. Neuronal activity in the monkey striatum during conditional visuomotor learning. Exp brain Res. 2003; 153: 190-6. https://doi.org/10.1007/s00221-003-1592-4 PMID: 14610634

71. Yamamoto S, Monosov IE, Yasuda M, Hikosaka O. What and Where Information in the Caudate Tail Guides Saccades to Visual Objects. J Neurosci. 2012; 32: 11005-11016. https://doi.org/10.1523/ JNEUROSCI.0828-12.2012 PMID: 22875934

72. Strecker RE, Steinfels GF, Abercrombie ED, Jacobs BL. Caudate unit activity in freely moving cats: effects of phasic auditory and visual stimuli. Brain Res. 1985; 329: 350-353. https://doi.org/10.1016/ 0006-8993(85)90548-7 PMID: 3978457

73. Caan W, Perrett DI, Rolls ET. Responses of striatal neurons in the behaving monkey. 2. Visual processing in the caudal neostriatum. Brain Res. 1984; 290: 53-65. https://doi.org/10.1016/0006-8993 (84)90735-2 PMID: 6692139

74. Ding L, Gold Jl. Caudate Encodes Multiple Computations for Perceptual Decisions. J Neurosci. 2010; 30: 15747-15759. https://doi.org/10.1523/JNEUROSCI.2894-10.2010 PMID: 21106814

75. Fisher SD, Reynolds JNJ. The intralaminar thalamus-an expressway linking visual stimuli to circuits determining agency and action selection. Front Behav Neurosci. 2014; 8: 1-7.

76. Kimura $\mathrm{M}$, Yamada $\mathrm{H}$, Matsumoto $\mathrm{N}$. Tonically active neurons in the striatum encode motivational contexts of action. Brain Dev. 2003; 25 Suppl 1: S20-3.

77. van der Meer MAA, Kalenscher T, Lansink CS, Pennartz CMA, Berke JD, Redish AD. Integrating early results on ventral striatal gamma oscillations in the rat. Front Neurosci. 2010; 4: 1-12.

78. Rueda-Orozco PE, Robbe D. The striatum multiplexes contextual and kinematic information to constrain motor habits execution. Nat Neurosci. 2015; 18: 453-462. https://doi.org/10.1038/nn.3924 PMID: 25622144

79. Wilson CJ. The Sensory Striatum. Neuron. 2014; 83: 999-1001. https://doi.org/10.1016/j.neuron. 2014.08.025 PMID: 25189207

80. Ragozzino KE, Leutgeb S, Mizumori SJ. Dorsal striatal head direction and hippocampal place representations during spatial navigation. Exp Brain Res. 2001; 139: 372-376. https://doi.org/10.1007/ s002210100795 PMID: 11545476

81. Mizumori SJY, Ragozzino KE, Cooper BG. Location and head direction representation in the dorsal striatum of rats. Psychobiology. 2000; 28: 441-462. 
82. van der Meer MAA, Redish AD. Theta Phase Precession in Rat Ventral Striatum Links Place and Reward Information. J Neurosci. 2011; 31: 2843-2854. https://doi.org/10.1523/JNEUROSCI.4869-10. 2011 PMID: 21414906

83. van der Meer MAA, Redish AD. Low and High Gamma Oscillations in Rat Ventral Striatum have Distinct Relationships to Behavior, Reward, and Spiking Activity on a Learned Spatial Decision Task. Front Integr Neurosci. 2009; 3: 9. https://doi.org/10.3389/neuro.07.009.2009 PMID: 19562092

84. Doll BB, Duncan KD, Simon DA, Shohamy D, Daw ND. Model-based choices involve prospective neural activity. Nat Neurosci. 2015; 18: 767-772. https://doi.org/10.1038/nn.3981 PMID: 25799041

85. Rennó-Costa C, Lisman JE, Verschure PFMJ. The mechanism of rate remapping in the dentate gyrus. Neuron. 2010; 68: 1051-8. https://doi.org/10.1016/j.neuron.2010.11.024 PMID: 21172608

86. Dupret $D$, Pleydell-Bouverie B, Csicsvari J. Rate remapping: when the code goes beyond space. Neuron. 2010; 68: 1015-6. https://doi.org/10.1016/j.neuron.2010.12.011 PMID: 21172603

87. DeCoteau WE, Thorn C, Gibson DJ, Courtemanche R, Mitra P, Kubota Y, et al. Learning-related coordination of striatal and hippocampal theta rhythms during acquisition of a procedural maze task. Proc Natl Acad Sci U S A. 2007; 104: 5644-5649. https://doi.org/10.1073/pnas.0700818104 PMID: 17372196

88. Lalla L, Rueda-Orozco P, Jurado-Parras M-T, Brovelli A, Robbe D. Local or Not Local: investigating the Nature of Striatal Theta Oscillations in Behaving Rats. Eneuro. 2017; 4: ENEURO.0128-17.2017. https://doi.org/10.1523/ENEURO.0128-17.2017 PMID: 28966971

89. Carmichael JE, Gmaz JM, van der Meer MAA. Gamma Oscillations in the Rat Ventral Striatum Originate in the Piriform Cortex. J Neurosci. 2017; 37: 7962-7974. https://doi.org/10.1523/JNEUROSCl. 2944-15.2017 PMID: 28716962

90. Bernard C. Editorial: Experimental Bias in Electrophysiological Studies. Eneuro. 2017; 4: ENEURO.0432-17.2017. https://doi.org/10.1523/ENEURO.0432-17.2017 PMID: 29279862

91. Halnes G, Mäki-Marttunen T, Pettersen KH, Andreassen OA, Einevoll GT. Ion diffusion may introduce spurious current sources in current-source density (CSD) analysis. J Neurophysiol. 2017; 118: 114120. https://doi.org/10.1152/jn.00976.2016 PMID: 28298307

92. Bédard C, Destexhe A. Generalized cable theory for neurons in complex and heterogeneous media. Phys Rev E-Stat Nonlinear, Soft Matter Phys. 2011; 88: 1-15. https://doi.org/10.1103/PhysRevE.88. 022709 PMID: 24032866

93. Taverna S, Canciani B, Pennartz CMA. Membrane properties and synaptic connectivity of fast-spiking interneurons in rat ventral striatum. Brain Res. 2007; 1152: 49-56. https://doi.org/10.1016/j.brainres. 2007.03.053 PMID: 17459351

94. Jones MW, Wilson MA. Phase precession of medial prefrontal cortical activity relative to the hippocampal theta rhythm. Hippocampus. 2005; 15: 867-73. https://doi.org/10.1002/hipo.20119 PMID: 16149084

95. Siapas AG, Lubenov EV, Wilson MA. Prefrontal phase locking to hippocampal theta oscillations. Neuron. 2005; 46: 141-51. https://doi.org/10.1016/j.neuron.2005.02.028 PMID: 15820700

96. Wilson CJ, Kawaguchi Y. The origins of two-state spontaneous membrane potential fluctuations of neostriatal spiny neurons. J Neurosci. 1996; 16: 2397-410. PMID: 8601819

97. Kondabolu K, Roberts EA, Bucklin M, McCarthy MM, Kopell N, Han X. Striatal cholinergic interneurons generate beta and gamma oscillations in the corticostriatal circuit and produce motor deficits. Proc Natl Acad Sci. 2016; 201605658. https://doi.org/10.1073/pnas.1605658113 PMID: 27185924

98. Bartos $\mathrm{M}$, Vida I, Jonas $\mathrm{P}$. Synaptic mechanisms of synchronized gamma oscillations in inhibitory interneuron networks. Nat Rev Neurosci. 2007; 8: 45-56. https://doi.org/10.1038/nrn2044 PMID: 17180162

99. Fries P, Nikolić D, Singer W. The gamma cycle. Trends Neurosci. 2007; 30: 309-16. https://doi.org/ 10.1016/j.tins.2007.05.005 PMID: 17555828

100. Schulz JM, Pitcher TL, Savanthrapadian S, Wickens JR, Oswald MJ, Reynolds JNJ. Enhanced highfrequency membrane potential fluctuations control spike output in striatal fast-spiking interneurones in vivo. J Physiol. 2011; 589: 4365-81. https://doi.org/10.1113/jphysiol.2011.212944 PMID: 21746788

101. Klausberger T, Magill PJ, Márton LF, Roberts JDB, Cobden PM, Buzsáki G, et al. Brain-state- and cell-type-specific firing of hippocampal interneurons in vivo. Nature. 2003; 421: 844-8. https://doi.org/ 10.1038/nature01374 PMID: 12594513

102. Buzsaki G. Neuronal Oscillations in Cortical Networks. Science (80-). 2004; 304: 1926-1929. https:// doi.org/10.1126/science.1099745 PMID: 15218136

103. Delcasso S, Huh N, Byeon JS, Lee J, Jung MW, Lee I. Functional Relationships between the Hippocampus and Dorsomedial Striatum in Learning a Visual Scene-Based Memory Task in Rats. J Neurosci. 2014; 34: 15534-15547. https://doi.org/10.1523/JNEUROSCI.0622-14.2014 PMID: 25411483 
104. Fernández-Ruiz A, Oliva A, Nagy GA, Maurer AP, Berényi A, Buzsáki G. Entorhinal-CA3 Dual-Input Control of Spike Timing in the Hippocampus by Theta-Gamma Coupling. Neuron. 2017; 93. https:// doi.org/10.1016/j.neuron.2017.02.017 PMID: 28279355

105. Paxinos G, Watson C. The Rat Brain in Stereotaxic Coordinates. 7th ed. London: Academic Press; 2013

106. Takeuchi Y, Osaki H, Yagasaki Y, Katayama Y, Miyata M. Afferent Fiber Remodeling in the Somatosensory Thalamus of Mice as a Neural Basis of Somatotopic Reorganization in the Brain and Ectopic Mechanical Hypersensitivity after Peripheral Sensory Nerve Injury. eneuro. 2017; 4: ENEURO.034516.2017. https://doi.org/10.1523/ENEURO.0345-16.2017 PMID: 28396882

107. Schmitzer-Torbert NC, Redish AD. Task-dependent encoding of space and events by striatal neurons is dependent on neural subtype. Neuroscience. 2008; 153: 349-360. https://doi.org/10.1016/j. neuroscience.2008.01.081 PMID: 18406064 
A

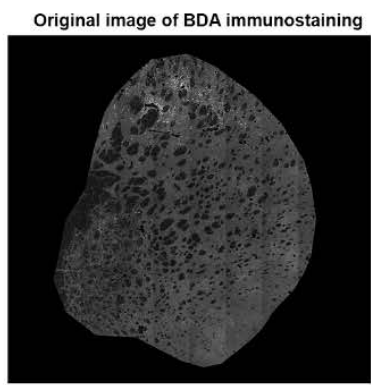

Original image of cFos immunostaining

B

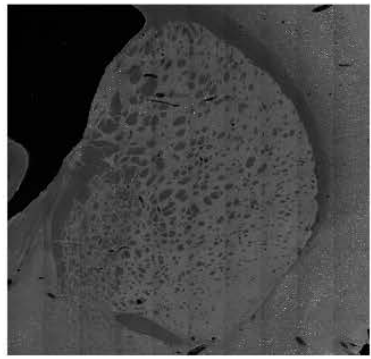

C

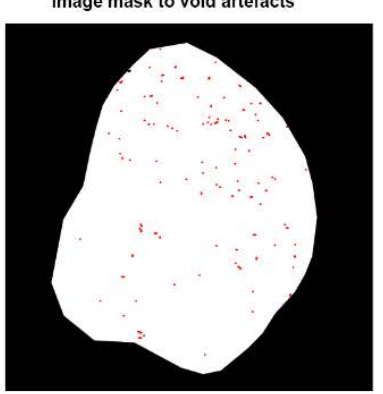

$\mathbf{F}$

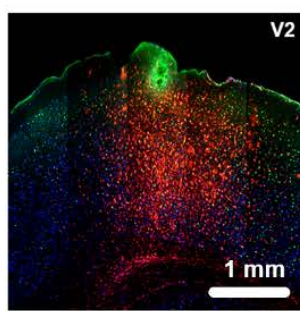

Thresholded image of BDA immunostaining
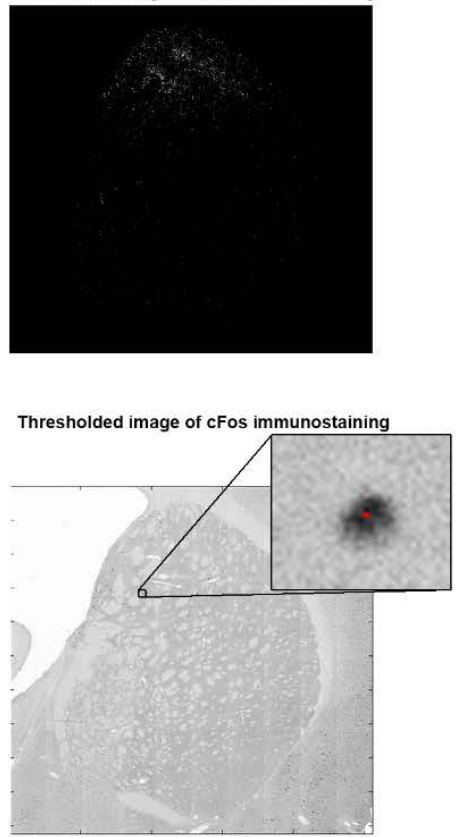

D

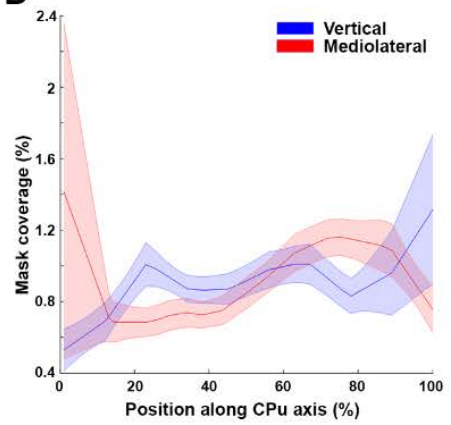

G

G

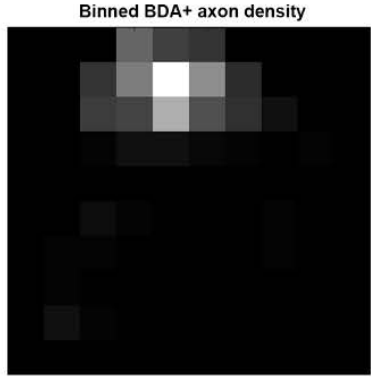

Binned cFos+ soma density

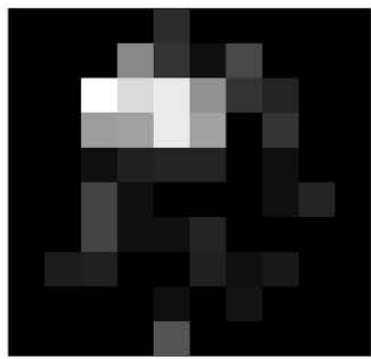

E

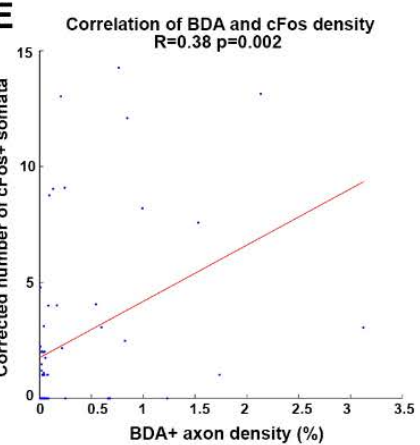

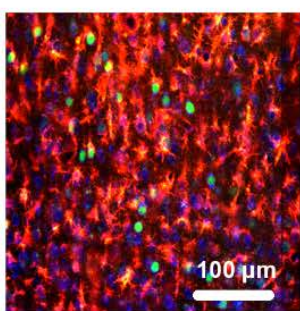
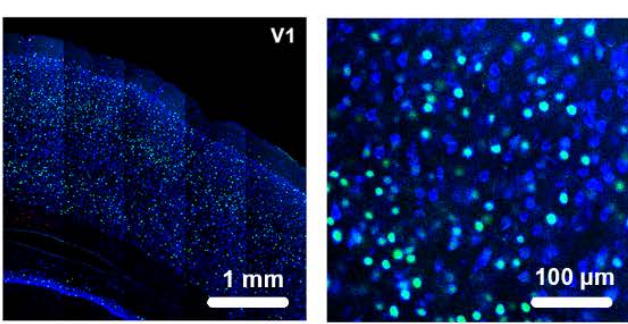

Fig S1. Steps of the automated detection of cFos+ cell and BDA labelled axon density and cortical cFos expression.

(A) Original tile-scan image of the BDA labelled axons on a representative example slice (left). Middle panel shows the intensity thresholded image, while right panel denotes the calculated binned axon density. (B) Original tile-scan of the cFos+ neuronal somata on the same slice (left), and its inverted version (middle). Inset of the middle panel shows a magnified image of a cFos+ neuron. Note the red dot placed at the center of the soma by the automated detection algorithm. Right panel shows the binned density of the automatically detected cFos+ neurons. (C) Manually generated image mask to avoid artefact detection. Black mask covers the non-CPu structures, while red masks cover the intra-CPu artefacts (e.g. noise, vessels, etc.). (D) Distribution of intra-CPU masks at all slices along the vertical (blue) and mediolateral (red) axes. Note, that the masks were distributed uniformly, and covered only approx. $1 \%$ of the total CPu area. (E) Correlation of the binned BDA and cFos density values of the representative slice shown on panels A-C. The correlation coefficient of the value pairs and the significance level of the correlation are displayed above the plot. (F,G) Small (left) and large (right) magnification photomicrographs of the V2 injection site of the BDA tracer (F) and the V1 (G) of a stimulated animal. Color channels are identical to those on Figure 2. Note the large number of cFos+ neurons in both regions as a result of the visual stimulation. 

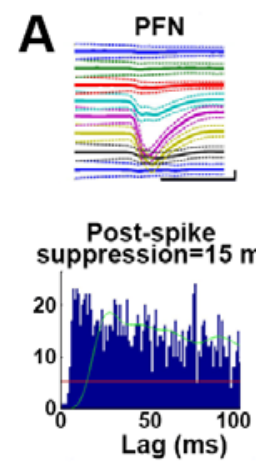
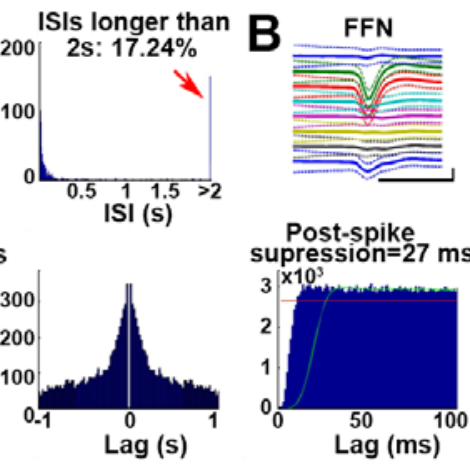

D

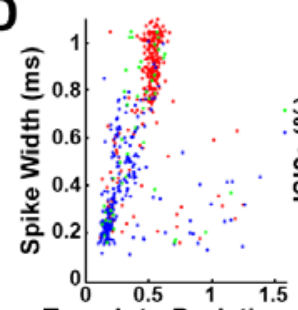

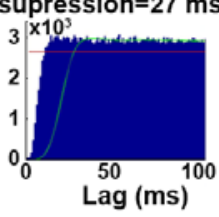

Lag (ms)

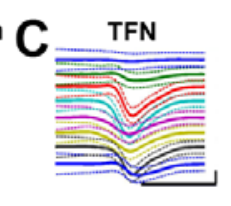

Post-spike Post-spike
supression $=55 \mathrm{~ms}$

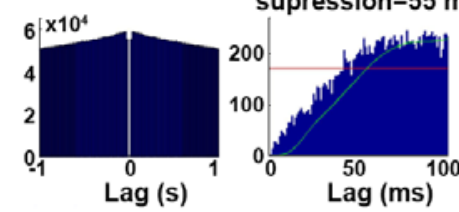

E

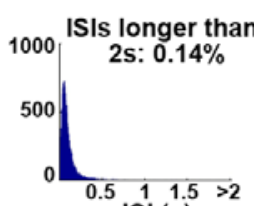

ISI (s)

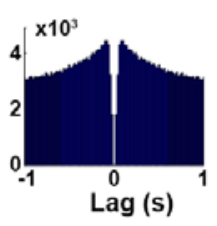

Fig S2. Basic electrophysiological properties and intrastriatal connectivity of the recorded CPu neurons.

(A - C) Waveform and spike train characteristics of a representative PFN (A), FFN (B) and TFN (C). Top left panels: Mean extracellular spike waveforms on the eight recording sites of the corresponding electrode shank. Top right panels: Distribution of interspike intervals, ISIs longer than two seconds (ISI2s) are aggregated. Note the high proportion of long ISIs for PFNs (red arrow). Bottom left and right panels display the post-spike suppression histograms and autocorrelograms, respectively. (D) Classification of the recorded neurons based on the features of their spike waveforms (left panel) and spike trains (right panel). (E) Proportion of the recorded PFNs (64\%), FFNs (22\%), TFNs (8\%) and unclassified neurons (6\%) among all recorded single units. 
A

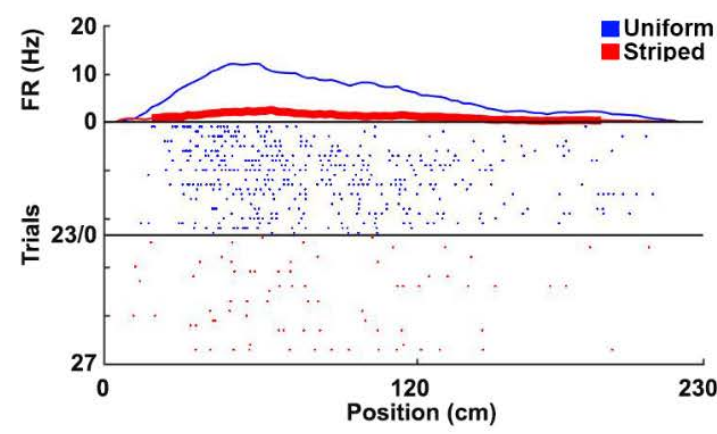

B
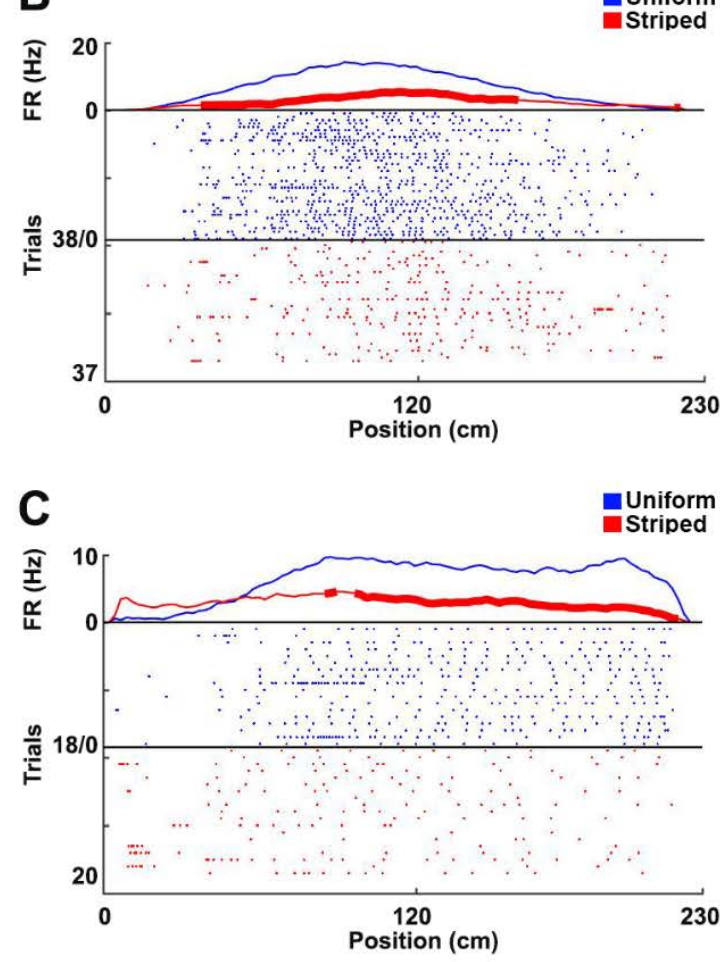

D

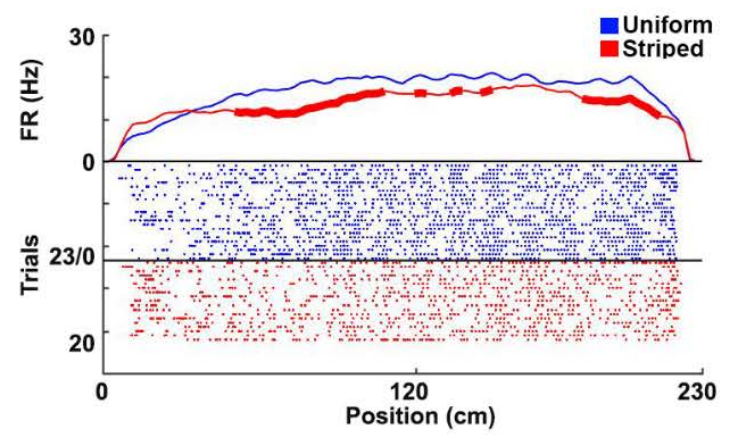

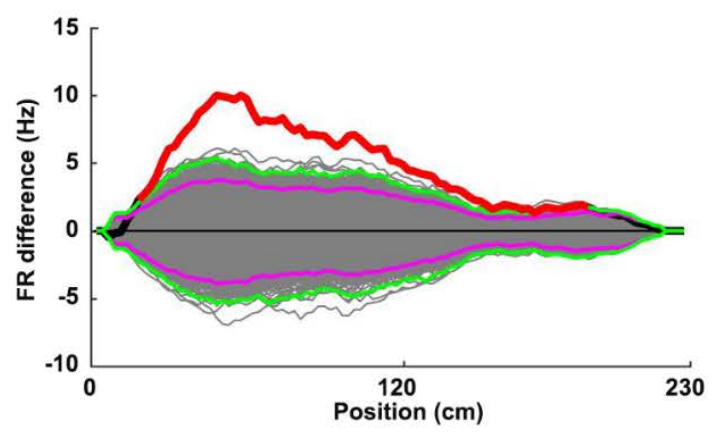
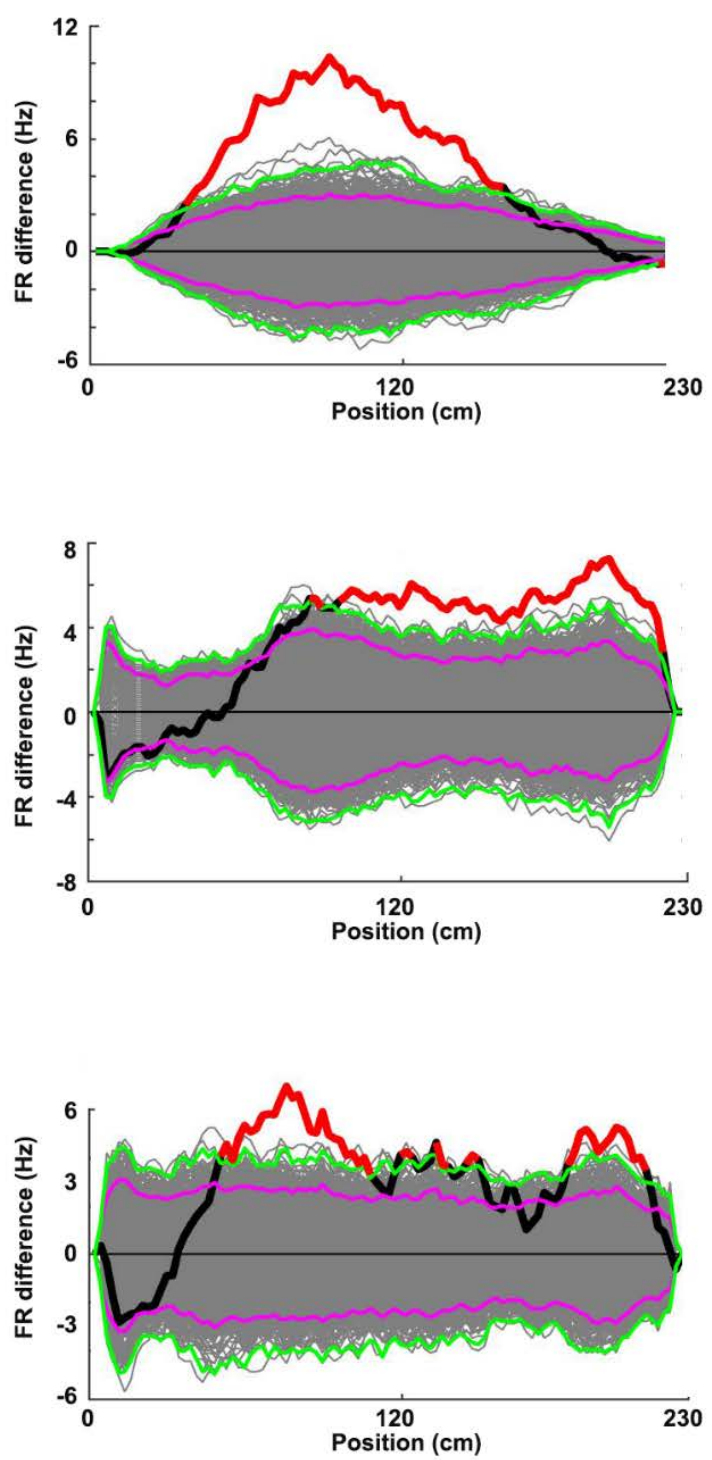

Fig S3. (A-D) Rate remapping of four example aVis+ neurons.

The left panels of each example shows the spatially resolved firing patterns in the uniform and striped environments (blue and red, respectively). Right panels show the details of statistical testing of the space-resolved visual modulation. The thick black lines show the real firing rate differences in the two environments, while the grey curves denote the label shuffled surrogate firing pattern differences. Purple and green lines depict the 'local' and 'global' significance thresholds, respectively. Spatial locations braking the global significance limits are marked with thick red lines in both the left and right panels. 


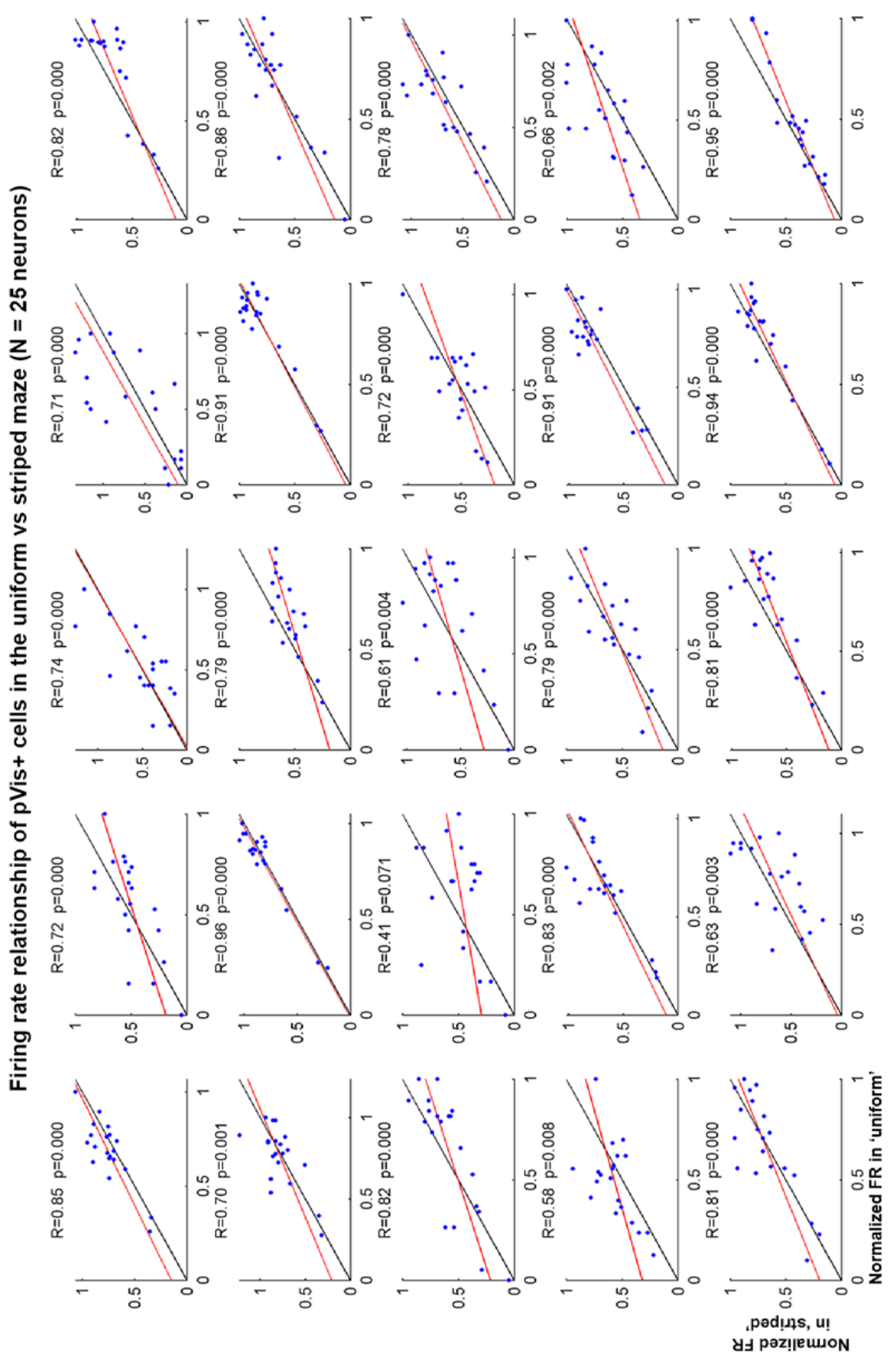

Fig S4. Firing rates of the pVis+ cells in the 'striped' vs 'uniform' maze.

The plots are showing the comparison of the normalized firing rates of each pVis+ neurons in the uniform (abscissa) and in the striped maze (ordinate). Identity and regression lines are shown in black and red, respectively. Correlation coefficients and significance levels are shown above each plot. To form the firing rate data points only those consecutive trials were included, where the condition was changing from 'uniform' to 'striped' or vice versa. Firing rate values were normalized by using the maximum rate during the 'uniform' trials. 


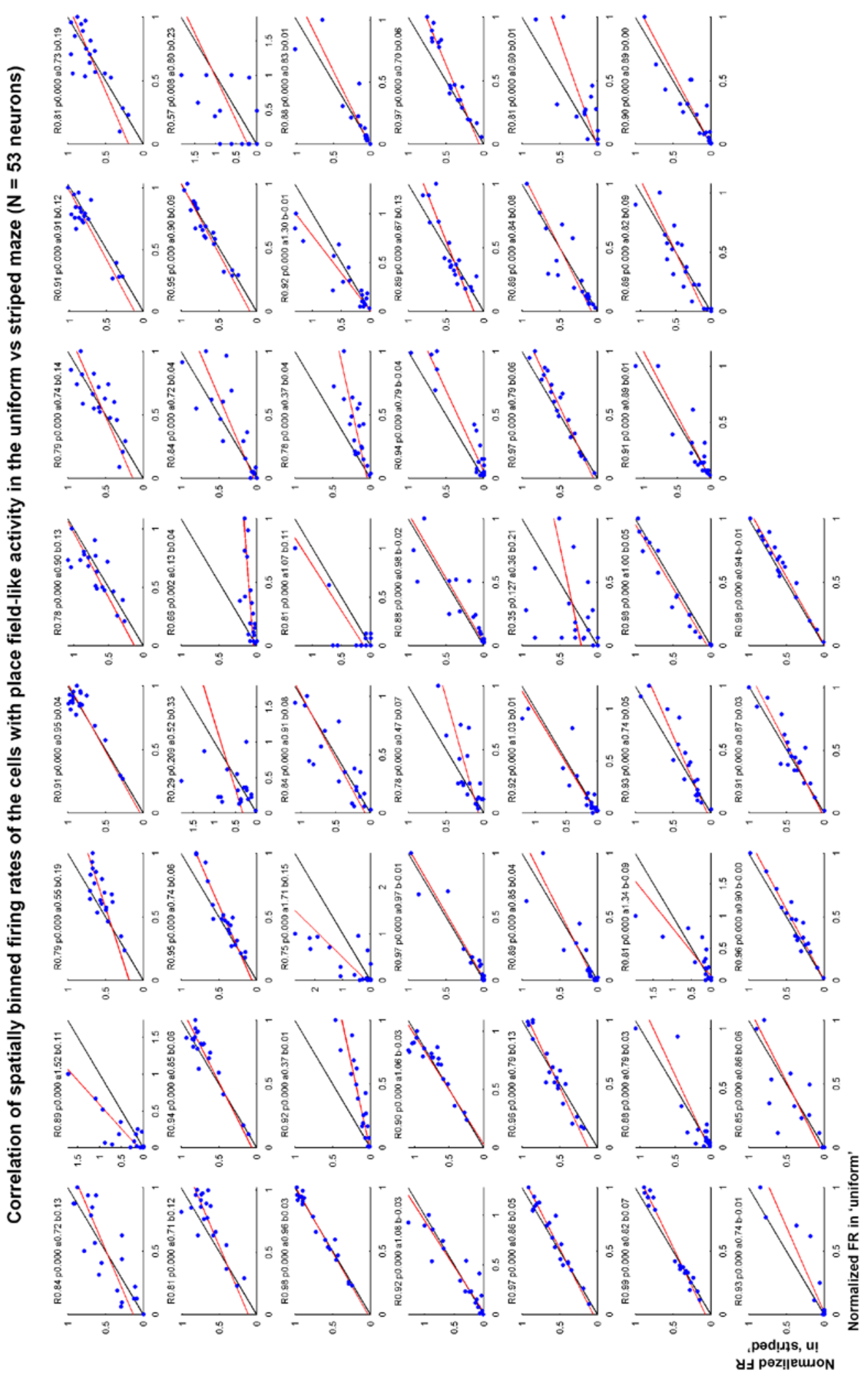

Fig S5. Comparison of place preference of the neurons expressing place field-like activity in the 'uniform' and the 'striped' environment. The plots show the comparison of the normalized firing rates of each place cell-like neurons in the uniform (abscissa) and in the striped maze (ordinate). Identity and regression lines are shown in black and red, respectively. Correlation coefficients, significance levels and the coefficients of the regression lines (' $a$ ' and ' $b$ ', where $y=a \cdot x+b$ ) are shown above each plot. To form the firing rate data points only those consecutive trials were included, where the condition was changing from 'uniform' to 'striped' or vice versa. Firing rate values were normalized by using the maximum rate during the 'uniform' trials. 
A

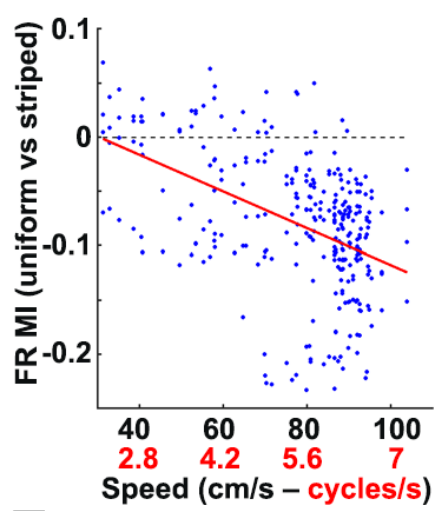

E

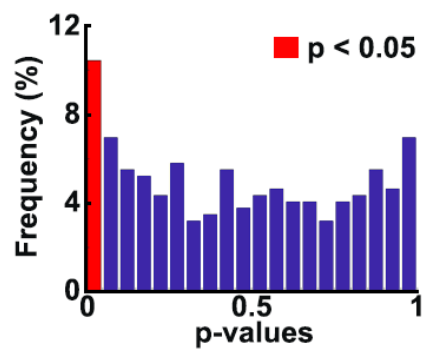

G

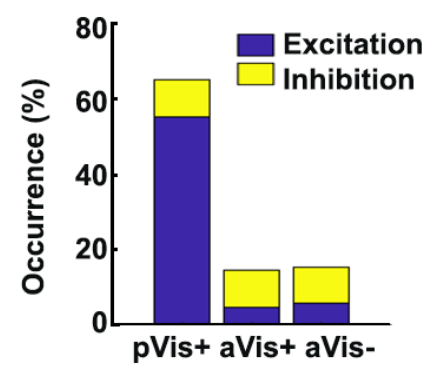

B

C
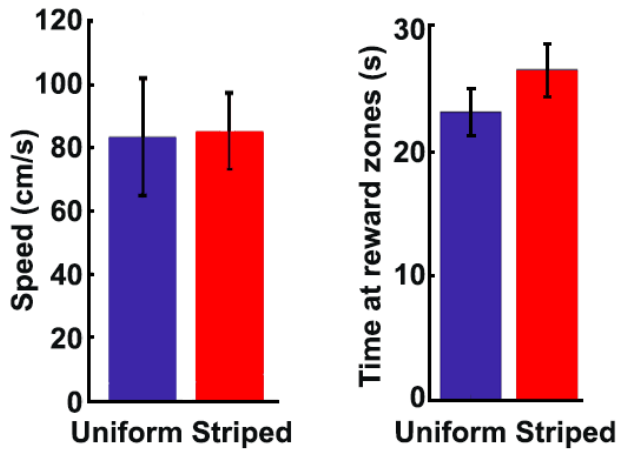

F

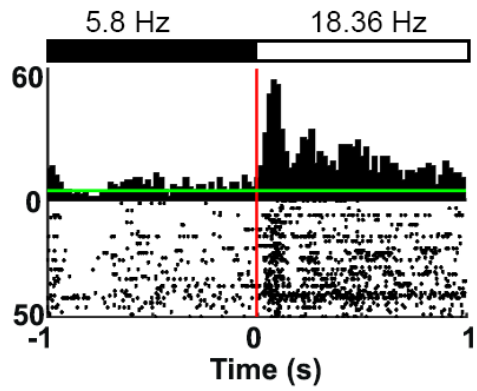

H

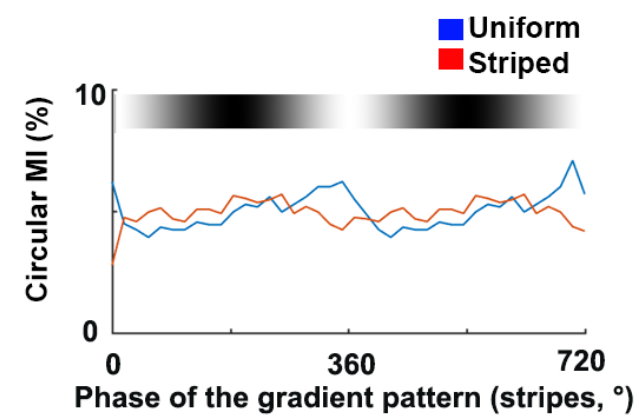

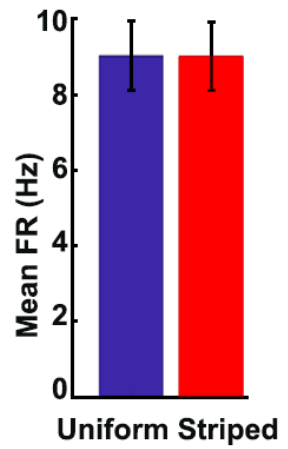

D
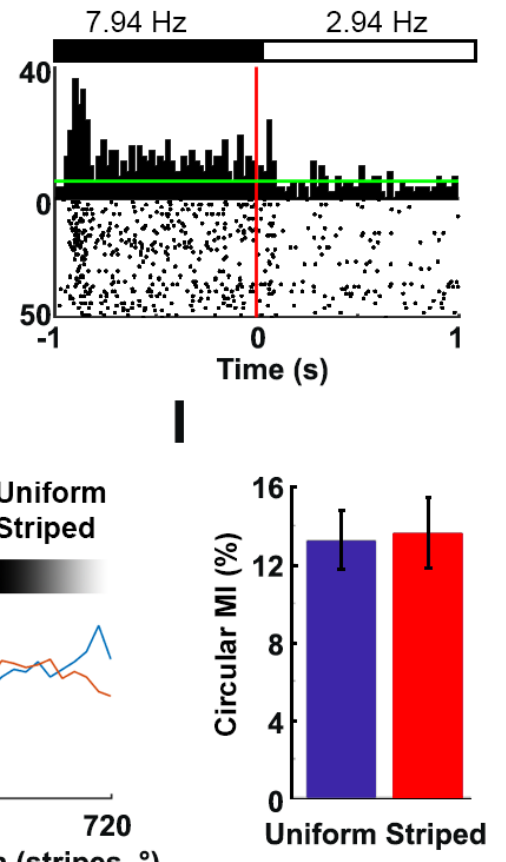

Fig S6. Response profiles of the aVis+ neurons in relation to space, context, velocity and luminance.

(A) Modulation indices of the rate remapping depend on the temporal velocity of the perceived visual motion, determined by the running velocity. Slower speeds elicited weaker decrease (i.e. weaker modulation) of the firing rates. (B) Comparison of the mean running speeds in the striped and uniform environments. (C-E) Analysis of aVis+ neurons' activity patterns in the reward zones, where the rats were not running, thus no visual motion was perceived, but they still observed the stationary patterns of the walls. (C) The average time spent at the reward zones in trials when uniform or striped pattern were presented were similar. (D) The firing rates of the aVis+ neurons were almost identical at the reward zones, with no respect to the pattern or context. (E) The distribution of the significance levels of the cell-by-cell comparison of the firing patterns depict that only a small minority of the aVis + neurons perceived the stationary patters different. (F) Two representative neurons excited (left) or inhibited (right) by the global brightness increase. (G) Percent of neurons responsive to global luminance change. Note that in contrast to the aVis+ neurons, more than half of the pVis + neurons were sensitive to brightness. (H) Response profile of a representative neuron triggered by the individual sinusoidal cycles of the grated pattern. Local luminance levels are shown above the plot; two full stripe cycles are shown for better visibility. Note that the stripe-phaseresolved firing pattern (red) is similarly uniform as the pattern in the uniform trials (blue). (I) Mean stripe-phase-resolved circular modulation indices of the aVis+ neurons were similar in the two conditions. 

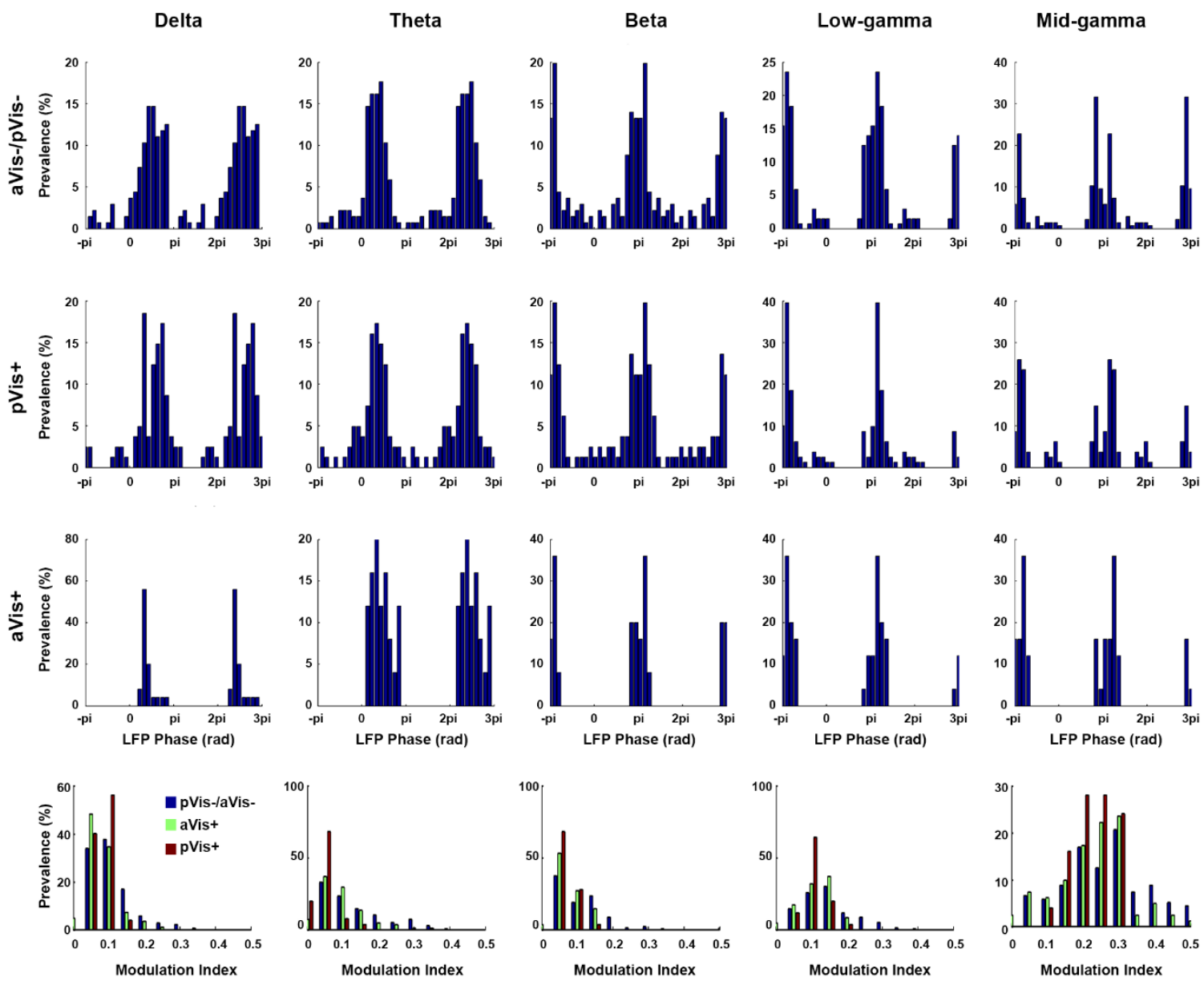

Fig S7. LFP phase coupling of the recorded neurons.

Preferred LFP phases are shown as histograms for pVis-/aVis- (top row), pVis+ (second row) and aVis+ (third row) neurons separately. Bottom row shows the comparison of phase coupling strength expressed as the distribution of the phase modulation indices for the three groups of neurons (for quantification, see Figure $5 \mathrm{C}$ ). Columns from left to right show the phase preferences for band pass filtered LFP signals in the delta, theta, beta, low-gamma and mid-gamma bands. For better visibility, the circular distributions are plotted twice (-180-540 deg) on the phase preference distribution histograms. 
A

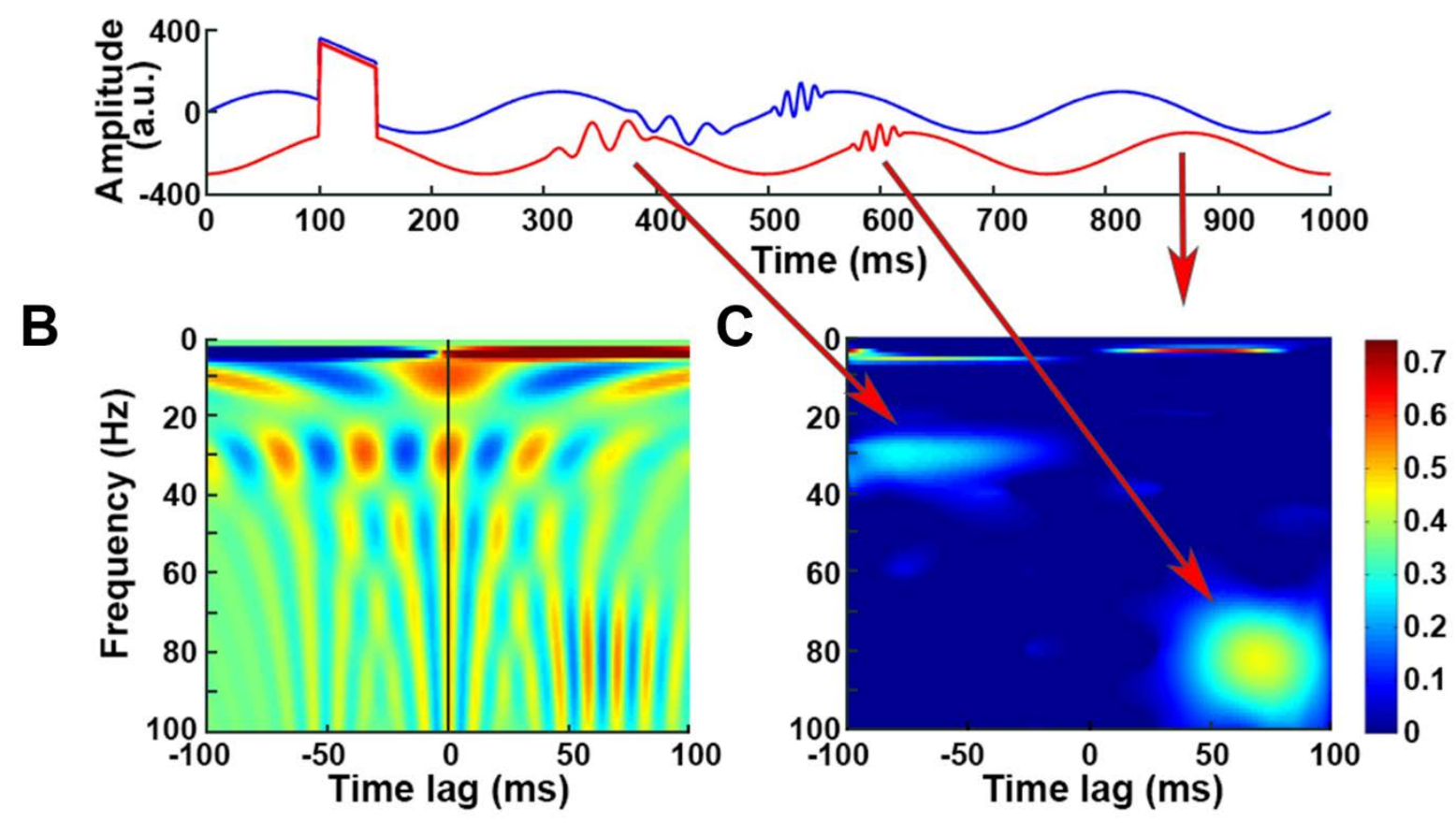

Fig S8. Demonstration of the concept of the novel time-lag and frequency resolved cross-correlation estimation method. (A) A demonstrative example of two artificial LFP signals with a phase delayed continuous theta oscillation, two time-delayed, discrete gamma bursts in the low and high gamma band, and a coincident square-wave resembling a recording artefact. (B) Raw time and frequency resolved cross-correlograms constructed from individual cross-correlograms calculated after iterative filtering for each frequency band. (C) The same time and frequency resolved cross-correlogram after the suppression of the zero-lag correlation components. Note the emergence of three domains (red arrows), representing the phase shifted theta oscillations and the gamma bursts with the appropriate time lags. Note also the lack of the artefact's spectral profile, which dominated the raw spectrogram on panel $B$. 
II. 


\title{
Large-scale, high-density (up to 512 channels) recording of local circuits in behaving animals
}

\author{
Antal Berényi, ${ }^{1,2,3}$ Zoltán Somogyvári ${ }^{2,4}$ Anett J. Nagy, ${ }^{3}$ Lisa Roux ${ }^{1}$ John D. Long, ${ }^{1}$ \\ Shigeyoshi Fujisawa, ${ }^{1,2,5}$ Eran Stark, ${ }^{1}$ Anthony Leonardo, ${ }^{6}$ Timothy D. Harris, ${ }^{6}$ \\ and György Buzsáki ${ }^{1,2,6}$ \\ ${ }^{1}$ New York University Neuroscience Institute, School of Medicine, New York University, New York, New York; ${ }^{2}$ Center \\ for Molecular and Behavioral Neuroscience, Rutgers, The State University of New Jersey, Newark, New Jersey; ${ }^{3}$ MTA-SZTE \\ “Momentum” Oscillatory Neuronal Networks Research Group, Department of Physiology, University of Szeged, Szeged, \\ Hungary; ${ }^{4}$ Wigner Research Center for Physics, Department of Theory, the Hungarian Academy of Sciences, Budapest, \\ Hungary; ${ }^{5}$ Laboratory for Systems Neurophysiology, RIKEN Brain Science Institute, Wako, Saitama, Japan; and ${ }^{6}$ Janelia \\ Farm Research Campus, Howard Hughes Medical Institute, Ashburn, Virginia
}

Submitted 4 November 2013; accepted in final form 10 December 2013

\begin{abstract}
Berényi A, Somogyvári Z, Nagy AJ, Roux L, Long JD, Fujisawa S, Stark E, Leonardo A, Harris TD, Buzsáki G. Large-scale, high-density (up to 512 channels) recording of local circuits in behaving animals. J Neurophysiol 111: 1132-1149, 2014. First published December 18, 2013; doi:10.1152/jn.00785.2013.-Monitoring representative fractions of neurons from multiple brain circuits in behaving animals is necessary for understanding neuronal computation. Here, we describe a system that allows high-channel-count recordings from a small volume of neuronal tissue using a lightweight signal multiplexing headstage that permits free behavior of small rodents. The system integrates multishank, high-density recording silicon probes, ultraflexible interconnects, and a miniaturized microdrive. These improvements allowed for simultaneous recordings of local field potentials and unit activity from hundreds of sites without confining free movements of the animal. The advantages of large-scale recordings are illustrated by determining the electroanatomic boundaries of layers and regions in the hippocampus and neocortex and constructing a circuit diagram of functional connections among neurons in real anatomic space. These methods will allow the investigation of circuit operations and behavior-dependent interregional interactions for testing hypotheses of neural networks and brain function.
\end{abstract}

local field potential; unit firing; monosynaptic connections; behaving rats and mice

ALTHOUGH THE EXPLORATION OF THE wiring diagram of brain networks is moving forward at an unprecedented scale (Lichtman and Denk 2011), and steady innovations in optogenetics provide a tool set for identification and manipulation of circuit components (Boyden et al. 2005; Lein et al. 2007; Madisen et al. 2012; Prakash et al. 2012; Tye and Deisseroth 2012; Yizhar et al. 2011), complementary methods for monitoring the activity of large numbers of neurons in multiple local circuits in the behaving animal are lagging (Alivisatos et al. 2013; Buzsáki 2004; Nicolelis et al. 1997). Yet, monitoring a statistically representative fraction of neurons of the investigated circuits in behaving animals is a prerequisite for understanding neuronal computation (Alivisatos et al. 2012, 2013; Buzsáki 2004; Carandini 2012; Nicolelis et al. 1997). Currently, recordings of individual neurons and local field potentials (LFPs) in local

Address for reprint requests and other correspondence: G. Buzsáki, NYU Neuroscience Institute, New York Univ., Langone Medical Center, East River Science Park, 450 East 29th St., 9th Fl., New York, NY 10016 (e-mail: gyorgy.buzsaki@nyumc.org). circuits at high temporal resolution are possible with wire or nanomachined microelectrodes ("silicon probes"; Blanche et al. 2005; Buzsáki 2004; Buzsáki et al. 2012; Du et al. 2011; Logothetis 2003; Wilson and McNaughton 1993). High-density probes can record from multiple cortical and subcortical structures in the freely behaving animal at the spatial resolution of single neurons (Blanche et al. 2005; Buzsáki 2004; Csicsvari et al. 2003; Du et al. 2011; Fujisawa et al. 2008; Montgomery et al. 2008). Furthermore, silicon probe recordings can be combined with optogenetic methods for the identification of neuron types and selective manipulation of local circuits (Anikeeva et al. 2012; Boyden et al. 2005; Royer et al. 2010; Stark et al. 2012).

Whereas silicon probe technology is poised to offer everlarger site numbers and smaller volume probes (Du et al. 2009, 2011), significant improvements and miniaturization are needed at the level of headstage interconnects, signal multiplexing, ultraflexible connection between the animal and the recording equipment, and signal processing (Du et al. 2011; Szuts et al. 2011; Vandecasteele et al. 2012). One critical aspect of miniaturization is the deployment of signal multiplexers. Previously used multiplexers have either low channel counts or limited high- or low-pass frequency characteristics for simultaneous recordings of both unit and LFP signals in the physiological range (Du et al. 2011; Harrison 2008; Olsson et al. 2005; Szuts et al. 2011; Viventi et al. 2011). Below, we present the development of a high-throughput integrated microelectronic system, including high-density, multiple-shank recordings of unit activity and LFP from multiple brain regions, using on-stage signal multiplexing methods. Applications are demonstrated in freely moving rats and mice, including extension to optogenetic manipulations.

\section{MATERIALS AND METHODS}

\section{Animal Surgery, Recording, and Behavioral Paradigms}

All experiments were approved by the Institutional Animal Care and Use Committee of New York University Medical Center and the Ethical Committee for Animal Research at the Albert Szent-Györgyi Medical and Pharmaceutical Center of the University of Szeged. The experimental protocol was in agreement with the European Communities Council Directive of November 24, 1986 (86/609/EEC) and the National Institutes of Health Guide for the Care and Use of Laboratory Animals. Five 
male Long-Evans rats (400-640 g; 3-10 mo old) were implanted with high-density, 256-site silicon probes under isoflurane anesthesia as described earlier (Vandecasteele et al. 2012). The probes and the polyimide interconnect cable were manufactured by NeuroNexus (http://www.neuronexus.com). In two rats, two probes were implanted; in one rat the probes were placed in the same hemisphere, whereas in the other they were placed symmetrically in the two hemispheres (Fig. 1). During surgery, the tips of the shanks are inserted into the superficial cortical layers or $\sim 1 \mathrm{~mm}$ above the intended subcortical target. After recovery, the probe is moved gradually until the target layer is reached using 70 - to $150-\mu \mathrm{m}$ rotations per day until most or all shanks record units and the probe has reached the desired target. The operated animals were housed in individual cages.

Neuronal activity in the neocortex and/or the hippocampus was recorded while the animals were running in a $240-\mathrm{cm}$ long linear maze, performing a delayed alternation task in a similar-sized T-maze for water reward, or freely exploring an open-field platform. The neuronal activity during rest/sleep in the home cage preceding and following the waking sessions was also recorded. The wide-band signal was low-pass filtered and downsampled to $1,250 \mathrm{~Hz}$ to generate the LFP and high-pass filtered $(>0.8 \mathrm{kHz})$ for spike detection. Malfunctioning recording sites (due to high impedance, cross talk, and short circuit) were removed from the analysis. Operated mice (male, 8-20 wk) were also housed individually after surgery. Details of surgery, optogenetic methods, and behavioral tests are available in Stark et al. (2012).

\section{Histology}

Following the termination of the experiments, the animals were deeply anesthetized and transcardially perfused first with $0.9 \%$ saline solution

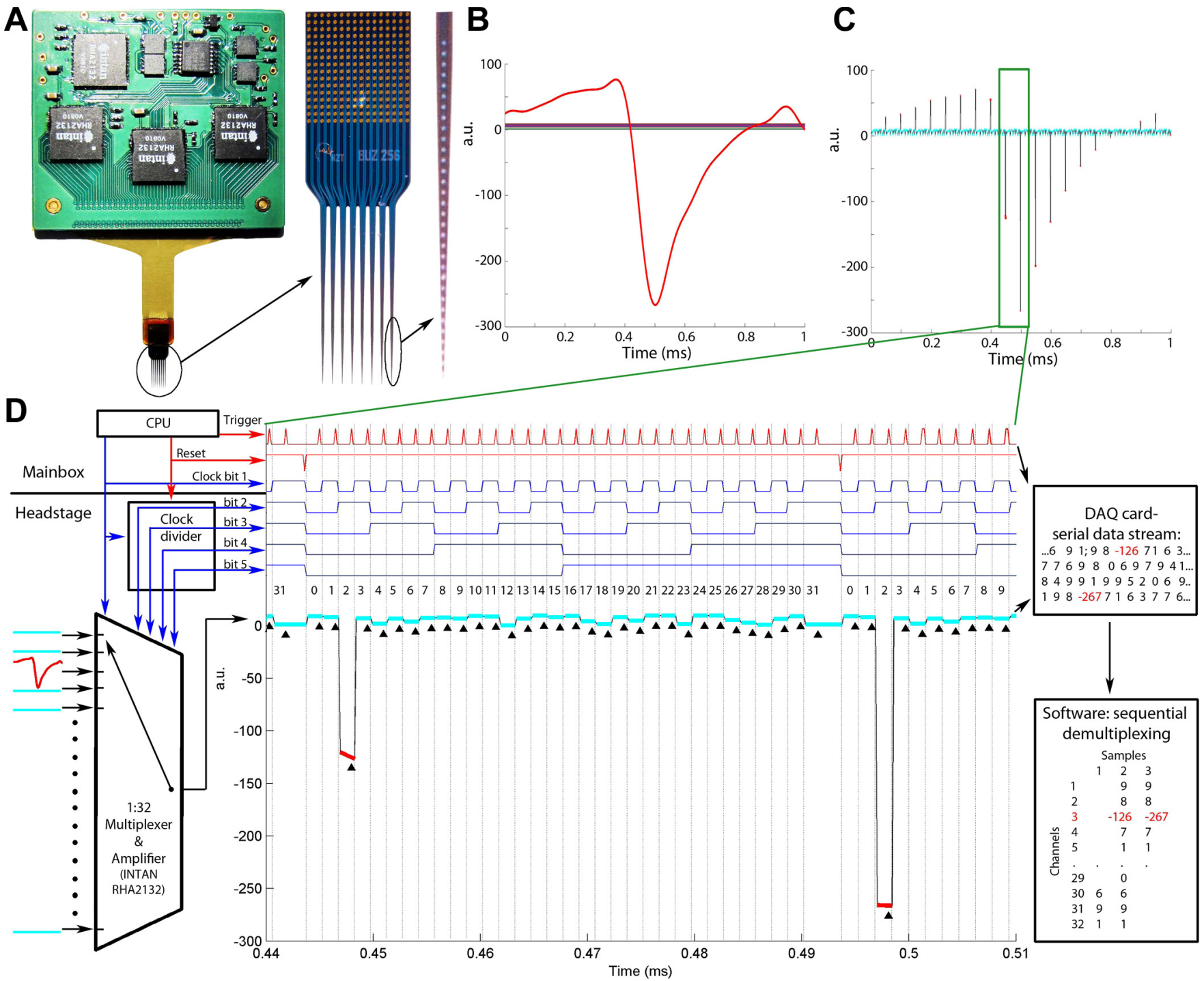

Fig. 1. System overview. A: high-channel-count ( 256 sites) silicon probe connected to a printed circuit board via a flexible polyimide ribbon cable. The printed circuit board contains 8 separate 32 -channel signal multiplexers ( 4 on each side) and accessory circuit elements. $B$ : a simulated headstage input signal illustrating a spike waveform on 1 of the 32 channels and various levels of direct current (DC) on the remaining 31 channels (1 ms). a.u., Arbitrary units. $C$ : time-shared multiplexed signal transmitting the 32 channels shown in $B$. D : circuit schematics and working principle of the multiplexer and a zoomed segment of the time epoch shown by rectangle in $C$. The multiplexer chip receives the input signals and is driven by both the clock signal of the main microcontroller and the complementary clock bits generated by the clock divider. The horizontal line separates circuits contained in the headstage and the main box. Middle part: zoomed segment of the multiplexed signal shown in $B$. The reset line resets the clock bits to " 00000 " after every 32 steps to ensure the proper channel order. The trigger signal is timed to sample the "tail" of each transmitted signal snippet (marked by black triangles). The 2 large steps (red) correspond to the 2 digital samples at the trough of the spike waveform on input channel 3. Right part: numerical representation of the analog-to-digital (A/D)-converted multiplexed line (readout from the A/D card) and its demultiplexed form after the software reconstruction of the digitized samples. CPU, central processing unit. 
followed by $4 \%$ formaldehyde solution. The brains were sectioned by a Vibratome (Leica) at $100 \mu \mathrm{m}$, parallel with the plane of the implanted silicon probes. Sections were DAPI-stained and mounted in Fluoromount (both Sigma-Aldrich). Some sections were immunostained against calbindin to determine the border between CA3 and CA2. The tracks were typically reconstructed from a few adjacent sections.

\section{Single-Unit Analyses}

Extracellular representations of action potentials were extracted from the recorded broad-band signal after high-pass filtering $(>800$ $\mathrm{Hz}$ ) by a threshold crossing-based algorithm. The dimensionality of the spike waveform representations on 32 contact sites of a given shank were reduced using principal component analysis, and the individual spikes were automatically clustered into groups with the lowest possible internal variance (i.e., representing action potentials generated by single neurons) using KlustaKwik (Harris et al. 2000). The generated cluster groups were manually refined by discarding multiunit clusters showing corrupted autocorrelograms. Groups with unstable firing patterns over time were also deleted. To compare the quality of single-unit cluster isolation under various conditions, cluster qualities were estimated using the following two conjunctive measures (Harris et al. 2000; Stark et al. 2012). 1) Cluster overlap in the high-dimensional feature space was estimated by the Mahalanobis distance (isolation distance, ID). 2) The ratio between the frequency of spikes present within 0- to 2-ms interspike interval (ISI) and those
A

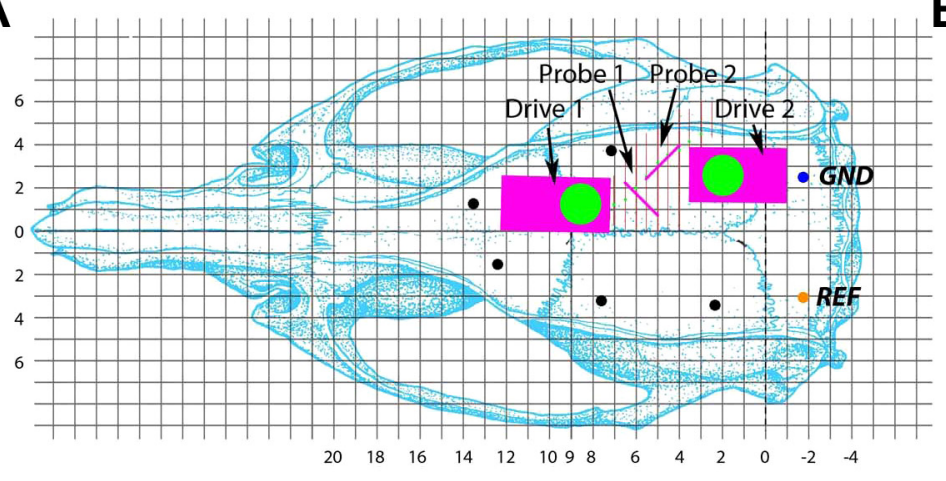

B

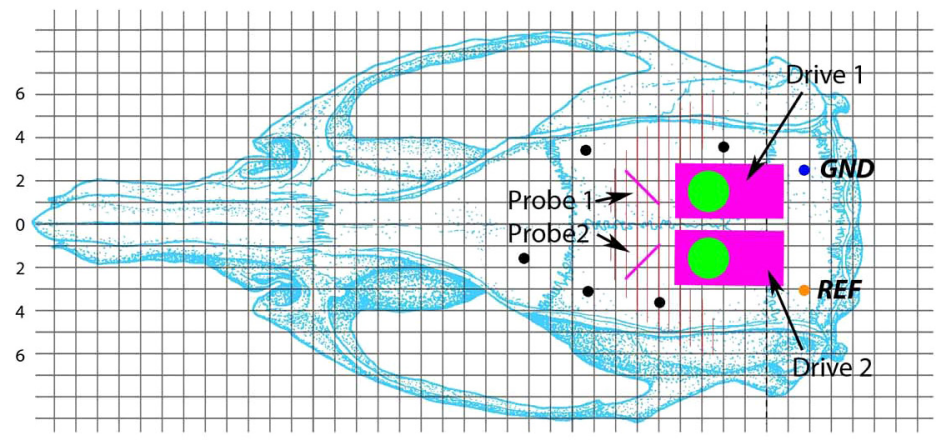

C
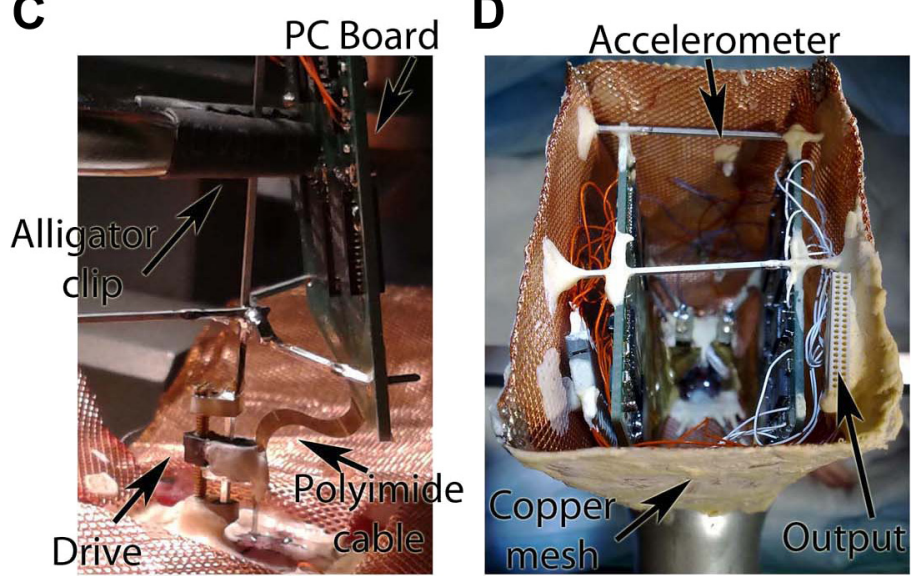

E
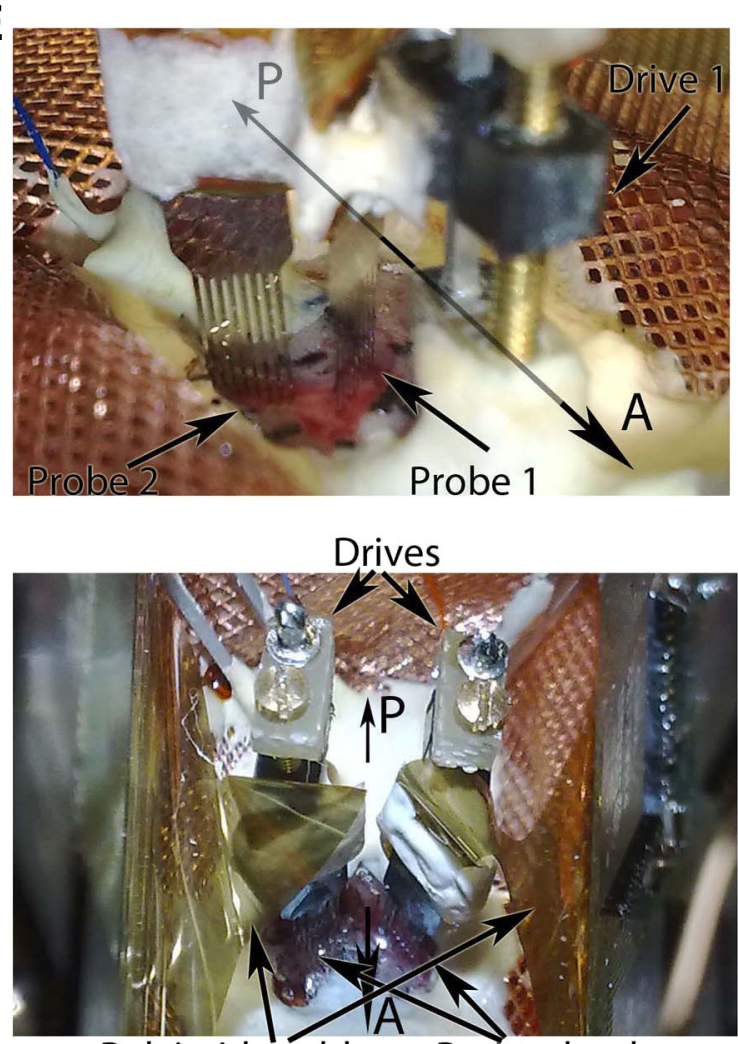

F
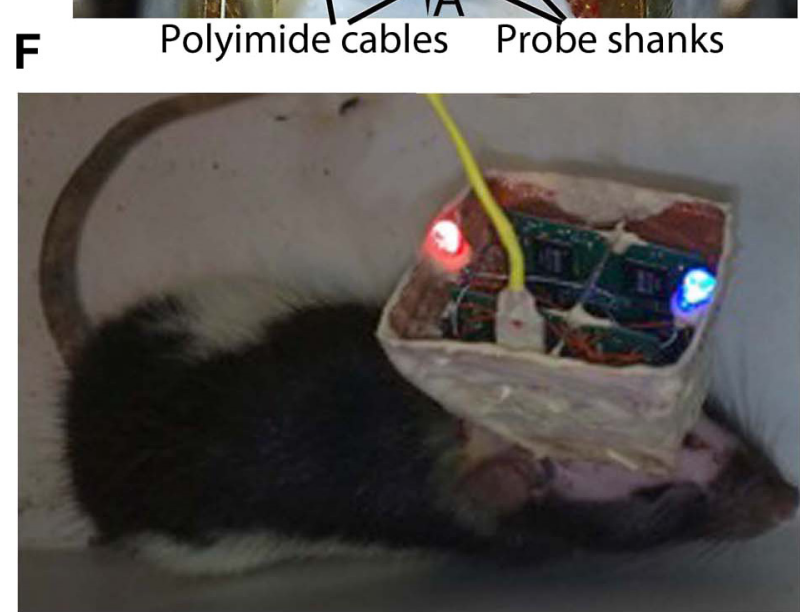

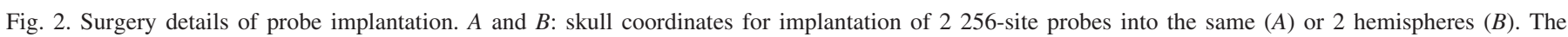

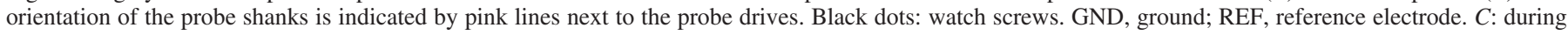

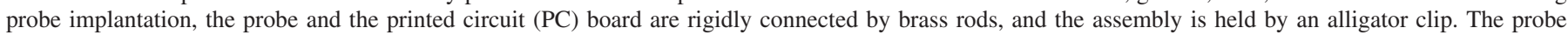

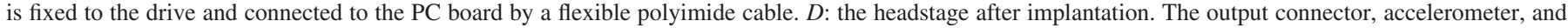

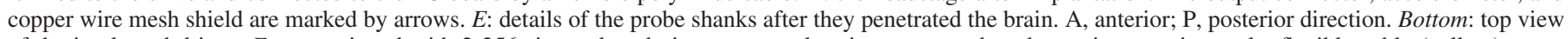
of the implanted drives. $F$ : rat equipped with 2 256-site probes during maze exploration connected to the equipment via an ultraflexible cable (yellow). 
at 0 - to 20-ms interval was defined as the ISI ratio and used as a measure of possible contamination of an isolated single unit by spikes of another unit. We used two levels of criteria to exclude contaminated clusters from our analyses. In the "permissive" approach clusters with either ISI ratio $<0.4$ or ID $>30$ were included, whereas in the "conservative" analyses clusters only with ISI ratio $<0.2$ and ID $>$ 40 were included.

The number of simultaneously recorded units (unit yield) was quantified as an average number of neurons per session per recording site overlapping with cellular layers.

For behavior-related analyses, the spatial position of the animal was sampled every $30 \mathrm{~ms}$ with $3-\mathrm{mm}$ resolution. For LFP phase-dependent circular metrics, the phase of each LFP frequency component was determined by Hilbert-transforming the 0-phase shift, digital filtered LFP $(2 \times 4$ th-order Butterworth infinite impulse response filter).

\section{Cross-Correlation Analysis}

Monosynaptic interactions of single units were determined by the examination of the cross-correlograms. Short-latency (1-5 ms), narrow (1-2 ms) peaks or troughs (monosynaptic excitation or inhibition, respectively) were deemed significant if they crossed a global threshold band determined from a surrogate data set of 1,000 jittered spike trains (99\% confidence interval, \pm 0 - to 4 -ms jittering with uniform distribution; Fujisawa et al. 2008). For monosynaptic interactionbased network mapping (Fig. 11), the permissive data set of single units was used since a moderate contamination of the unit clusters does not introduce spurious short-latency peaks. Spike contamination increases "noise" in the cross-correlogram and, in fact, decreases the probability of finding monosynaptically connected neuron pairs.

\section{Spike-Triggered LFP Maps}

Sixty-millisecond long, low-pass filtered $(<600 \mathrm{~Hz})$, perispike LFP traces centered at the spike occurrences of the spike train of selected neurons were extracted. The LFP segments for each recording site were high-pass filtered $(20$ and $5 \mathrm{~Hz}$ for run and sleep sessions, respectively; 0-phase shift $2 \times 4$ th-order Butterworth infinite impulse response filter). The purpose of the higher cutoff frequency for run sessions was to eliminate the large-amplitude theta fluctuation. The filtered perispike segments were averaged across multiple spikes (usually a few thousand occurrences for run sessions and a few hundred for sharp wave-ripple segments). The mean perispike LFPs of the 256 or 512 recording channels were reordered in 2 dimensions (2-D) to match with the anatomic layout of the recording sites on the silicon probe, forming an activity map of the recorded structures. Consecutive data points in each segment were visualized as multiple frames of a movie using the following equation:

$\operatorname{MAP}_{i j}=\frac{\tan ^{-1}\left(\frac{\mathrm{LFP}_{i j}}{\operatorname{maxLFP}} \times z f\right)}{\tan ^{-1}(z f)}, i \in N\{1, \ldots, 8\}, j \in N\{1, \ldots, 32\}$

D

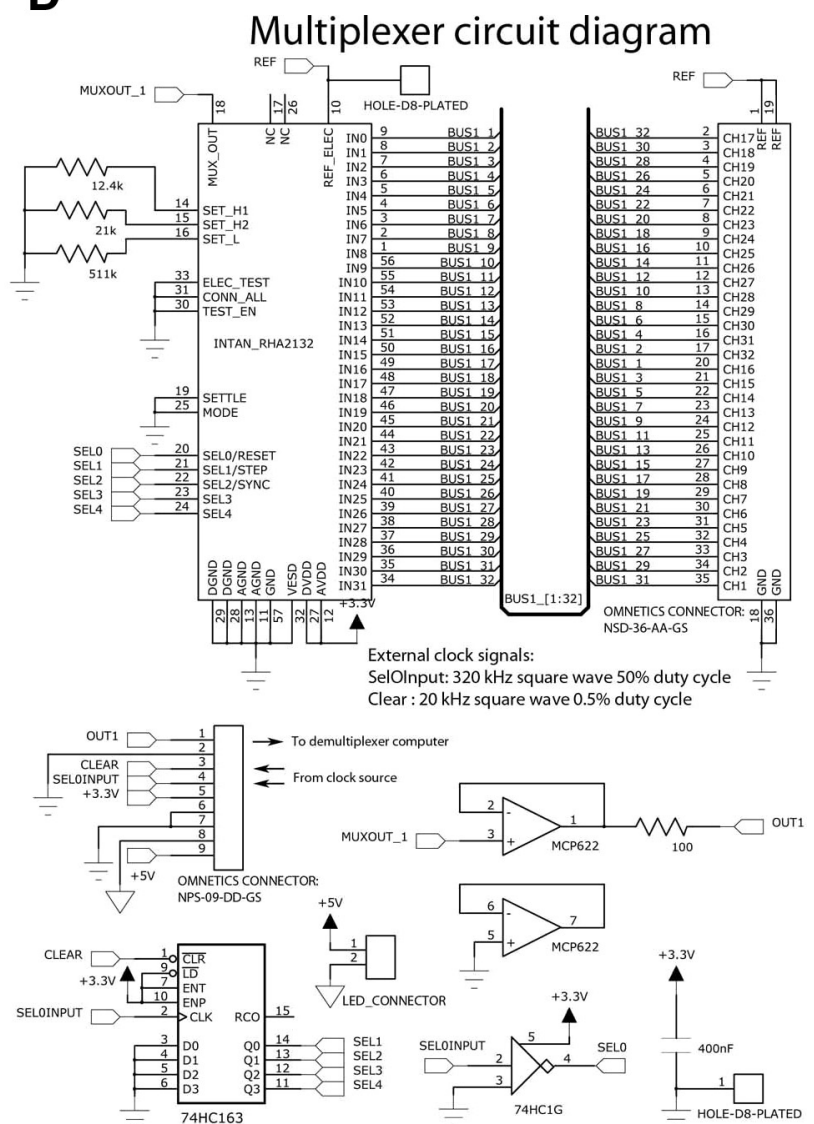

Fig. 3. Headstage multiplexers. $A$ and $B$ : 32- and 64-channel multiplexers with high-density Omnetics connectors. $C$ : 64-site probe bonded to a $64-$ channel multiplexer. $D$ : circuit schematics representing the electrical components and wiring scheme of the 32-channel multiplexer. Top: wiring diagram of the Intan RHA-2132 multiplexer chips and the high-density Omnetics connector for electrode interfacing. The cutoff frequencies of the low- and high-pass filters are set with the 3 resistors on the left. Bottom: supplementary electronics to provide clock bits and buffer the signal. Top row, left to right: 9-pin Omnetics connector interfaces with the main box; high-speed, dual-buffer operational amplifier. Bottom row: synchronous clock divider chip; external light-emitting diode (LED) power port for position tracking; clock inverter chip with Schmitt trigger; decoupling capacitor. 

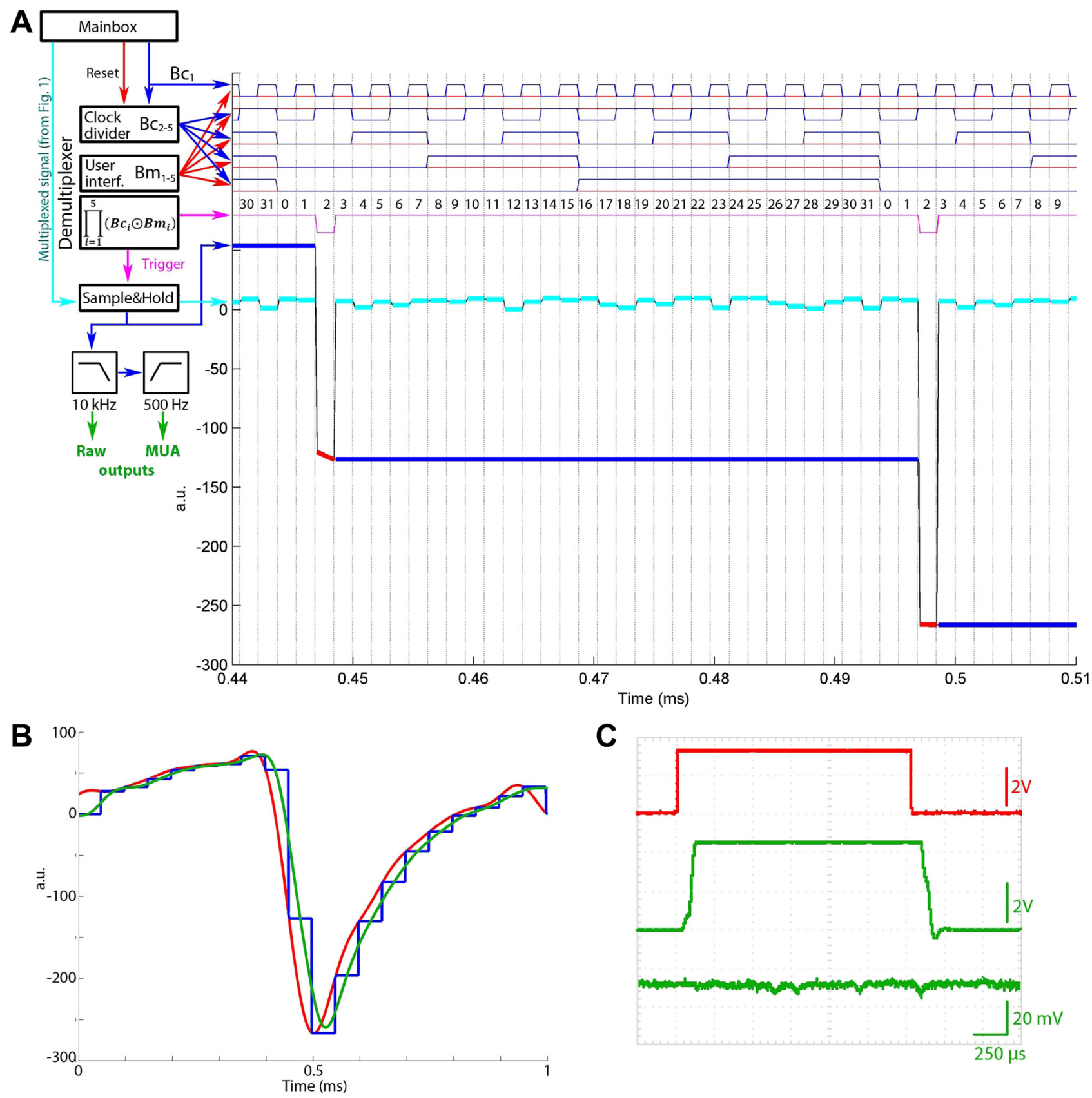

C

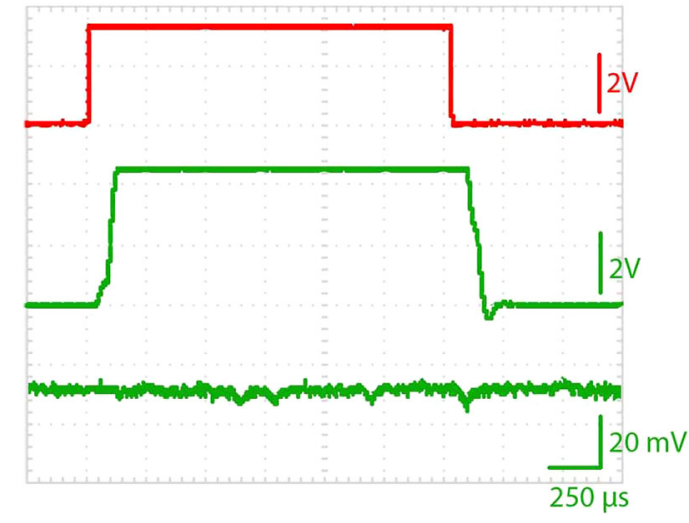

D
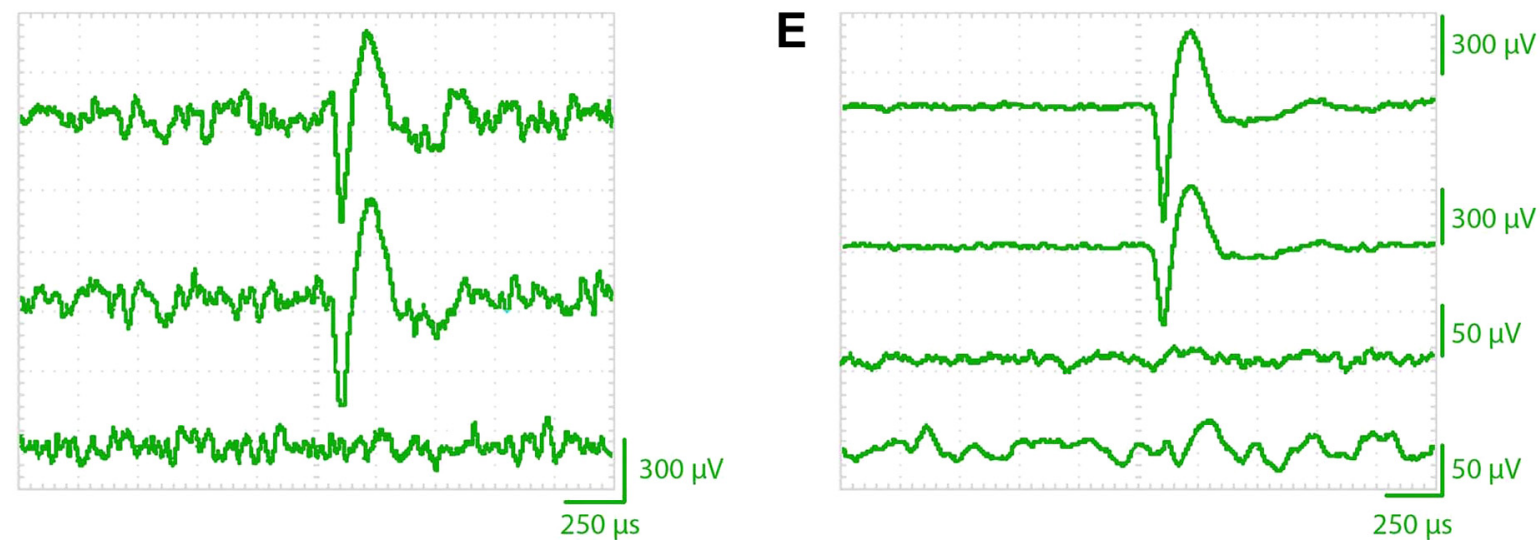
where $\mathrm{MAP}_{i j}$ is the transformed activity value of the $i$ th shank and $j$ th contact site to be displayed, $\mathrm{LFP}_{i j}$ is the filtered mean LFP value of a given channel, and $z f$ is an arbitrary zooming factor. This transformation helped to visualize the smooth activity patterns while relatively suppressing the gross changes. The generated activity map (heat map) frames were further smoothed in space by cubic spline interpolation (10× upsampling) and in time by a $150-\mu$ s (3-sample) wide-moving average filter. A schematic of the anatomic layers was overlaid on each frame based on the histological verification of electrode location.

\section{Mapping High-Frequency Power}

The LFP signals were filtered by a narrow Gaussian band-pass filter (peaks at $300 \mathrm{~Hz}, \mathrm{SD}=10 \mathrm{~Hz}$ ), and the power was summed up for 1 -s-long periods. Several other high-passed signal bands $(>300 \mathrm{~Hz})$ provided similar results. In the hippocampus, the selected periods typically contained a sharp wave-ripple event to maximize the presence of unit firing since the goal was to identify the somatic layers. Several other frequency bands and bandwidths were tested, and the $300-\mathrm{Hz}$ band was found empirically the most effective to outline the cell body layers (Ray and Maunsell 2011). This band was also less sensitive than higher frequencies due to the wide amplitude range of the isolated units that vary from site to site. The high-frequency power distribution effectively outlined the CA1 and CA 3 pyramidal layers and the dentate area. In addition, the high-frequency power map agreed well with the locations of the clustered neurons. The predicted positions of the cell body layers were in good agreement with the anatomically reconstructed tracks of the probe shanks.

\section{Coherence Analysis}

Using coherence as similarity measure, an interaction energy-based clustering was implemented to identify cell layers. Every site served as a reference against all of the other referred sites. The resulting values were clustered using a gradient-descent algorithm so that each site was merged with that cluster for which the resulting coherence gain after merging was the largest. Starting from random initial assignments, the clustering algorithm formed stable but fewer clusters corresponding to a local energy minimum. Energy of cluster $A$ is defined as:

$$
E^{A}=\frac{-1}{N^{A}} \sum_{i, j \in A} \mathrm{C}_{i j}
$$

where $C_{i j}$ is the coherence between $i$ th and $j$ th sites and $N^{A}$ is the number of recording sites in cluster $A$. The energy gap between two different assignments to clusters $A$ and $B$ of site $i$ is:

$$
\Delta E_{i}^{A B}=\frac{1}{N^{B}} \sum_{j \in B} C_{i j}-\frac{1}{N^{A}} \sum_{k \in A} C_{i k}
$$

If the energy gap is positive, site $i$ moves into cluster $B$, otherwise it remains in cluster $A$. Since the method results only local minima of energy and stochastic components, such as the random initial condition and update order affects, clustering consistency was verified by repeating the process several times. Small $(<3$ sites in a cluster) and scattered clusters (typically representing bad channels) were deleted, and their sites were included into the majority cluster of their immediate neighborhood. This clarification was done either before or after merging the coherence clusters and multiunit activity (MUA) map. The resulting clusters effectively differentiated the different anatomic layers of the hippocampus (Fig. 5). The same coherence similarity method was used in the neocortex to differentiate the superficial, middle (layer IV), and deep layers of the neocortex (Fig. 8).

\section{Merging the MUA and Coherence Cluster Maps}

MUA map was thresholded at $70 \%$ of its maximum to define the cellular layers (CA1 and CA3 pyramidal and dentate gyrus granular). These sites were deleted from the coherence clusters to define new clusters. As a result, the CA1 and CA3 pyramidal layer and the granular layer became different clusters, designated as cell body layer clusters.

\section{Coastline Display}

In a different display, each site was connected to the most coherent sites on the neighboring shanks, which connected forward to the most coherent site of the next shank and so on. The recording positions were slightly and randomly scattered to assist visualizing the different lines crossing the same points. The connecting lines were colored on the basis of the cluster identity of their seed.

\section{Estimation of Recording Quality and Comparison with a Commercially Available Nonmultiplexing Recording System}

To obtain an objective measure of the recording quality of our multiplexing system, we performed the following analyses. The inputreferred noise of the recording channels was measured by shortcircuiting all inputs of the Intan chip (http://www.intantech.com) to the reference channel. The power spectrum of a 10-s-long recording was whitened after performing Fourier transformation. To evaluate the quality of recorded LFP and units, several consecutive, 10-minlong sessions (typically during immobility and sleep) were recorded from the hippocampus with a Buzsaki32 four-shank silicon probe (NeuroNexus). The recordings were made with either our multiplexed system (test) or a commercially available nonmultiplexed system (control) in an alternating sequence. The control system consisted of a headstage (gain $=20 \times$; HST/32V-G20; Plexon, Dallas, TX) connected by a 40-wire Litz cable (same length as in the cable of the multiplexer) to a Digital Lynx 16SX analog-to-digital (A/D) converter (Neuralynx, Bozeman, MT). The multiplexed signals were sampled at $20 \mathrm{kS} / \mathrm{s}$, whereas the Digital Lynx used $32 \mathrm{kS} / \mathrm{s}$ sampling. Pearson linear correlation was calculated across all combinations of channels (496 pairs) and visualized as a correlation matrix. A mean correlation

Fig. 4. Demultiplexer circuit. A: working principle of the real-time demultiplexer. The clock signal from the main box (Fig. 1) is successively halved 4 times to produce 4 subsequent clock bits (blue trace, $\mathrm{Bc}_{1 . .5}$ ). A clock-bit-mask pattern set by the user interface (interf.; $\mathrm{Bm}_{1 . .5}$, red traces) is pairwise-compared (XNORed) with the clock bits, and the results are logically ANDed. The output of this logical operation is the trigger (pink trace), which switches the sample-and-hold circuit so that when the clock bits match the preset mask the circuit works as a relay (1/32nd of the running cycle); otherwise, it holds the last sampled voltage (31/32nd of the running cycle). The output of the sample-and-hold circuit is shown as red and blue lines for the sampling and holding periods, respectively. The example demultiplexes channel 2. The demultiplexed signal trace is low-pass filtered to remove the step functions and optionally high-pass filtered at $500 \mathrm{~Hz}$ to separate unit firing from the local field potential (LFP). MUA, multiunit activity. $B$ : temporal delay of the demultiplexing process. Red trace: original input waveform; blue trace: output of the sample-and-hold circuit; green trace: low-pass filtered signal at the output of the demultiplexer. $C$ : signal transmission characteristics of the demultiplexer for a large-amplitude step function. Red trace: original input signal; upper green trace: demultiplexed waveform; lower green trace: demultiplexed signal on an adjacent channel in the multiplexed sequence. Note the different amplitude scales for the traces. $D$ : single trace examples of a demultiplexed unit. The top 2 traces represent 2 waveforms representing 2 distinct projections of the spike onto 2 adjacent recording sites of the probe. The bottom trace shows the signal recorded on neighboring channel in the multiplexed stream. $E$ : spike-triggered average waveforms of the neuron shown on $D$. Note the lack of cross talk in both the temporal (incomplete signal level settling during multiplexing or demultiplexing, 3rd trace) and spatial (cross talk across leads, 4th trace) domain. 
value of the entire 10-min-long recording was calculated for channels on the same shank (within-shank correlation) and for channels located on different shanks (across-shank correlation). To estimate the contribution of higher-frequency components in the correlation, we repeated the analysis twice after passing the signals through a low-pass fourth-order Butterworth zero-phase lag digital filter (arbitrarily chosen $10-$ and $100-\mathrm{Hz}$ cutoff frequencies, respectively). The raw recorded signals were processed to extract single-unit spike trains and clustered automatically by the KlustaKwik program (Harris et al. 2000). To avoid subjectivity, the manual cluster refinement step was limited to eliminate the obvious noise clusters (waveforms with same amplitude and shape on every recording site of the given shank). The number and quality of the isolated single units were assessed using the following three criteria. In the first analysis, the number of automatically isolated nonnoise clusters was compared disregarding their isolation quality. In the second comparison, we measured the ratio of the number of isolated spikes and the number of noise waveforms to estimate the signal-to-noise ratio. Third, we used two levels of cluster quality measures (permissive and conservative; see above). The recorded waveforms of identical spike clusters recorded by the two systems were also compared after amplitude normalization and offline interpolation (upsampling) to $50 \mathrm{kS} / \mathrm{s}$.

\section{RESULTS}

\section{System Description}

Large-scale recordings from multiple single units, large spatial coverage, and limited tissue displacement/damage by the electrodes are competing conditions (Buzsáki 2004; Du et al. 2011). Our goal was to monitor single-unit and LFP activity patterns of neighboring neocortical regions and interconnected hippocampal subregions. To this end, we designed an 8-shank probe with 32 recording sites on each shank (Fig. 1A). The recording sites are arranged vertically at $50-\mu \mathrm{m}$ steps, providing sufficient spatial resolution for unit clustering (Csicsvari et al. 2003; Montgomery et al. 2008; Wilson and McNaughton $1993)$ yet large vertical coverage $(1,550 \mu \mathrm{m})$. Each recording site is $165 \mu \mathrm{m}^{2}$ and has an impedance between 1.3 and $3 \mathrm{M} \Omega$. The shanks are placed $300 \mu \mathrm{m}$ apart to eliminate simultaneous recording of neurons by adjacent shanks (Henze et al. 2000) and provide spatial coverage of adjacent neocortical modules or hippocampal regions (Csicsvari et al. 2003). Each shank is $15 \mu \mathrm{m}$ thick and tapered from a sharp tip to $96 \mu \mathrm{m}$ at the uppermost recording site to minimize tissue damage yet rigid enough to allow smooth penetration through brain tissue. The total volume of the probe shank, containing the recording sites, is comparable with traditional wire tetrodes (Wilson and McNaughton 1993). In contrast to the blunt tetrode, which often tears intracortical blood vessels during penetration, the tapered profile of the silicon probe shanks allows it to be moved up and down in the brain with continued yield of units. The probe is connected to a microdrive so that the probe sites can be advanced to the vicinity of the desired neurons (Vandecasteele et al. 2012; Fig. 2).

Signal multiplexing and processing. A major challenge of the effective use of silicon probes in small animals is to reduce the volume and weight of the instrumentation between the probe and the recording equipment. Multiple connectors, traditional preamplifiers and multistrand cables for each recording site are not viable for high-density probes; the large volume and weight of these components and the increased tension of the connecting cable significantly limit the behavior of the small rodent. In addition, using large numbers of individual external amplifiers is prohibitively costly and involves complex cabling. A logical solution to these problems is the deployment of time-division multiplexing (Harrison 2008; O1sson et al. 2005; Szuts et al. 2011; Viventi et al. 2011). To this end, we used 32-channel VLSI analog signal multiplexers developed specifically for brain recording applications (Harrison 2008; RHA2132; Intan). Eight multiplexers are soldered to a custom-designed printed circuit board, four on each side (Fig. $1 A$ ), to transmit a total number of 256 channels. To allow movement of the probe and preserve its small inertia, an ultraflexible polyimide-based cable was designed and served as an interconnect between the probe and the multiplexing headstage (Fig. 1A).

Each of the 256 electrical signals acquired by the silicon probe is amplified and band-pass filtered (gain $=200 \times$, cutoff frequencies of the high-pass and low-pass filters are $0.3 \mathrm{~Hz},-6$ $\mathrm{dB} /$ octave and $10 \mathrm{kHz},-18 \mathrm{~dB} /$ octave, respectively; Fig. $1 B$ ). The broadly tuned cutoff frequencies allow the recording of broad-band signals (LFP and unit activity simultaneously) from all recording sites. The signal multiplexer chips were programmed to switch sequentially between channels every 1.5 $\mu$ s so that a sweep of 32 channels corresponds to $48 \mu$ s (Fig. $1, C$ and $D$ ). To accomplish a $50-\mu$ s cycle length (20 complete scan cycles in a millisecond), the last channel of the 32-channel block was transmitted for $2 \mathrm{~ms}$ more before looping back to the 1 st channel of the subsequent cycle. The postloop 2- $\mu$ s time was used to protect against desynchronization of the multiplexing sequence. Potential desynchronization of the multiplexing and demultiplexing sequence may occur if the clock signal accidentally advances the sequence of the multiplexed signal. Such accidental "jumps" are avoided by resetting the binary counters to zero (binary code 00000 ) during the 2 - $\mu$ s postloop time. This clock supervision mechanism ensured that even if the clock code is corrupted for any reason, only 1 sample is misplaced in the sequence of the parallel-decoded channels. To reduce the wiring complexity of the printed circuit board interconnects, the 32 recording sites of a given shank did not correspond to a single multiplexer but were distributed among at least 4 chips. This had the added value that in case of a chip failure some recordings from all shanks remained available.

We found that the relatively high output impedance of the RHA2132 multiplexer chip was not sufficient to transmit signals reliably over long data cables $(>5 \mathrm{~m})$, typically needed in behaving experiments. To improve signal settling time and to circumvent the capacitive load of the cable, high-precision operation amplifiers (MCP622; Microchip; slew rate: $27 \mathrm{~V} / \mu \mathrm{s}$; Fig. $3 D$ ) were added in series to buffer the multiplexed signals. With the added buffering, the signal settled in $<100 \mathrm{~ns}$ to $1 \%$ precision after each switch of the multiplexer chip even when up to 15 -m-long cable, consisting of 36-gauge $(110-\mu \mathrm{m}) \mathrm{Litz}$ wires, was used. The ultralight and ultraflexible cable allowed for a free movement of the animal (Vandecasteele et al. 2012; Fig. 2F).

To achieve synchronous channel advancement on every multiplexer chip without adding further weight and surface area to the headstage, the common clock signal $(640 \mathrm{kHz}, 50 \%$ duty cycle) is generated by a programmable integrated circuit in the external controller box ("main box"). A 5-bit binary clock code was generated by a synchronous binary counter (74HC163; Texas Instruments) on the headstage to address the channels of the multiplexers within blocks of 32 . In the main 
box, the multiplexed signals were further amplified (gain $=2$, total gain $=400)$, and their baseline was corrected by the subtraction of a preset voltage value. The multiplexed signals (Fig. 1, $C$ and $D$ ) were sampled by a high-speed digital-toanalog converter (PCI-6133; National Instruments). Sampling

A

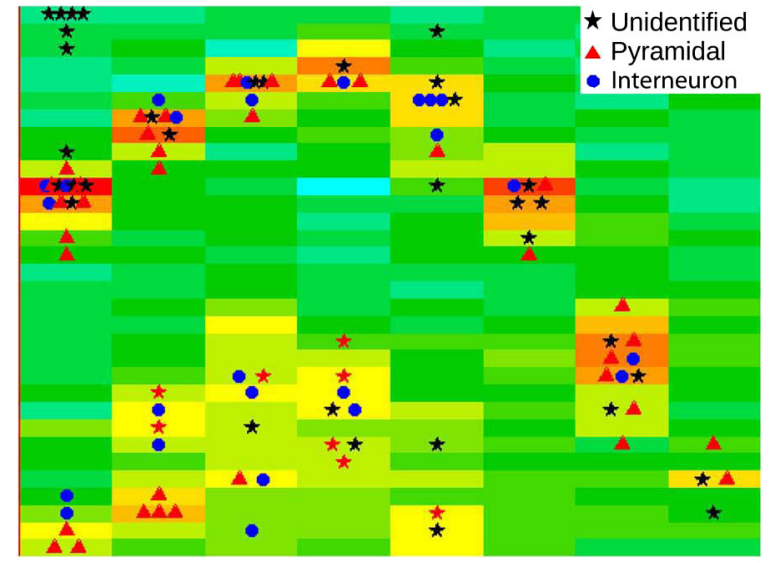

C

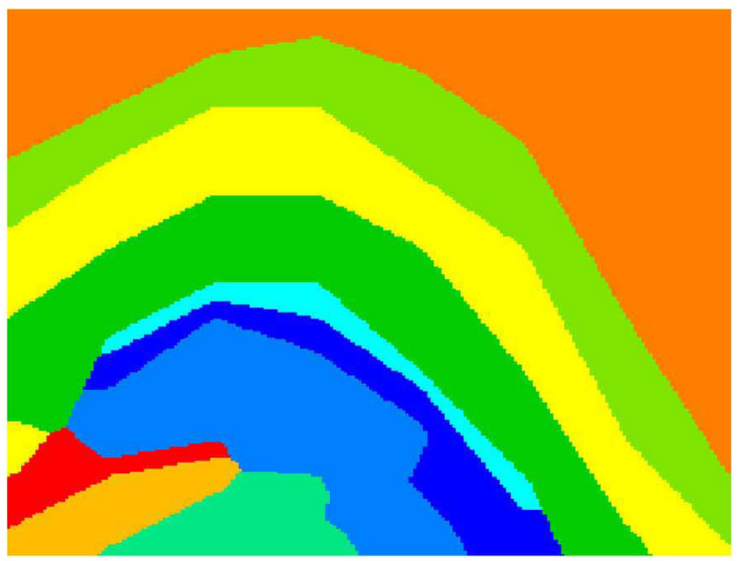

D

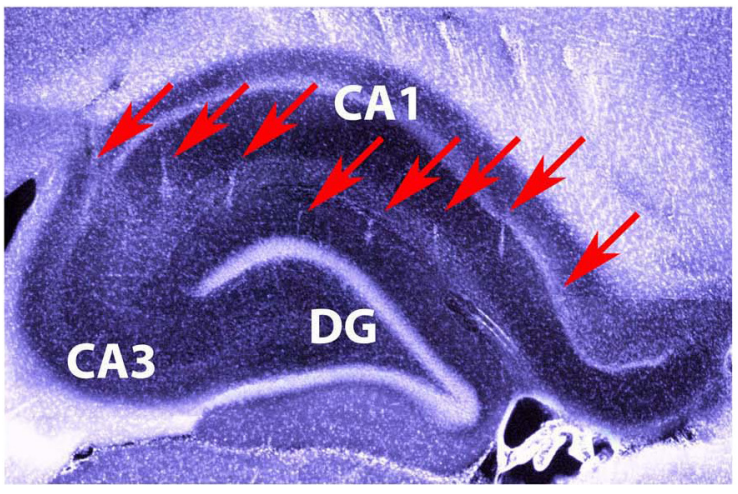

was initiated by the clock generator microchip of the main box 125 ns before the subsequent channel switch to exploit the available signal settling time maximally.

Software control. To generate the clock and trigger signals, the programmable integrated circuit was activated by the re-

B
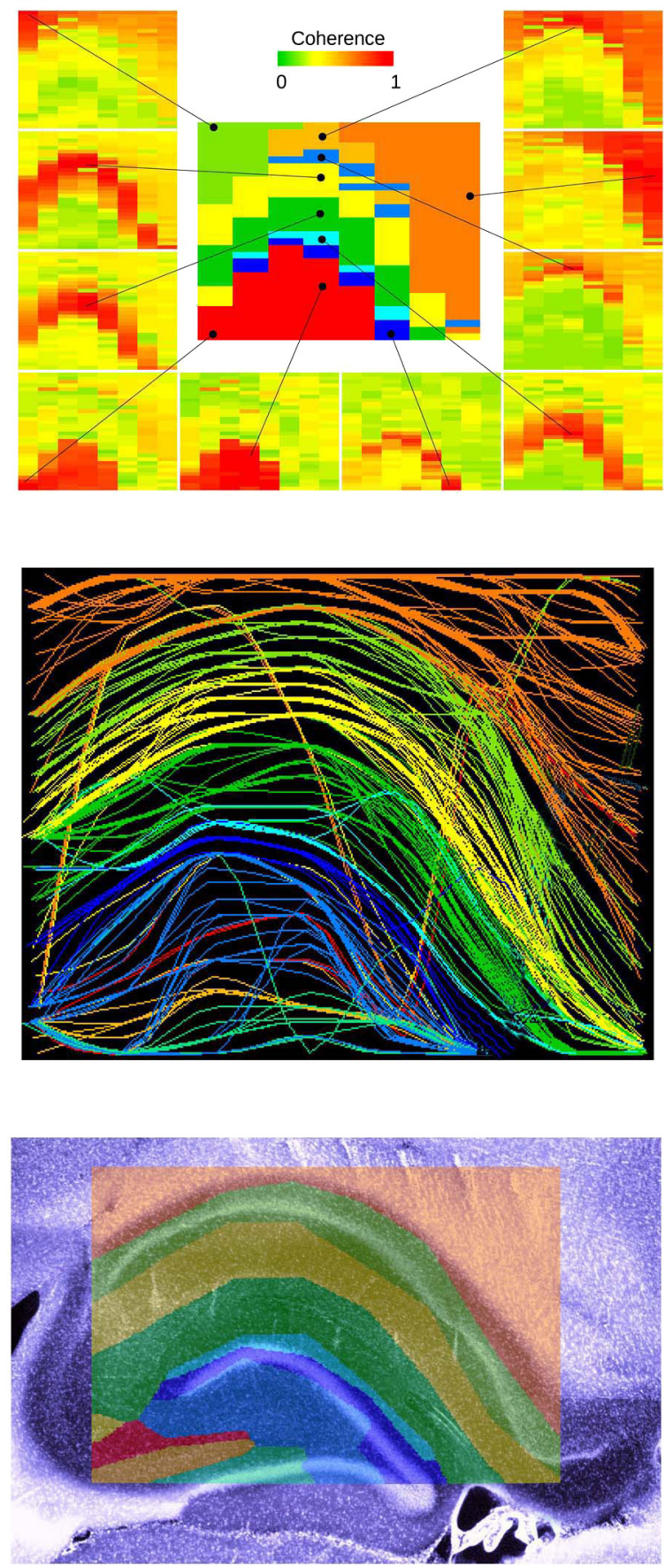

Fig. 5. Electroanatomy of the hippocampus. A: distribution of high-frequency power $(300 \pm 10 \mathrm{~Hz})$ on each of the 256 sites of the silicon probe. The $32 \times 8$ color matrix is a representation of the 256-site probe shown in Fig. $1 A$. Each rectangle represents a 300- $\mu \mathrm{m}$ (intershank distance) by $50-\mu \mathrm{m}$ (vertical intersite distance) area to mimic the 2-dimensional geometry of the probe coverage. Clustered neurons, assigned to the largest amplitude recording sites, are superimposed on the power map. $B$ : coherence maps of gamma activity $(30-90 \mathrm{~Hz})$. The 10 example sites (black dots) served as reference sites, and coherence was calculated between the reference site and the remaining 255 locations for a 1-s long recording segment (Fig. 7). C: composite figure of the combined coherence maps (see also Fig. 7). Left: 2-dimensional combined map of gamma coherence and high-frequency power distribution. Right: coastline map of layer-specific coherence contours. D: histological reconstruction of the recording tracks (arrows). The shifting of the tracks in the neocortex is due to a slight displacement of the neocortex/corpus callosum relative to the hippocampus during the tissue sectioning process. DG, dentate gyrus. Right: physiology-based map superimposed on the recording tracks. 
cording software only after the digital-analog data acquisition (DAQ) task was properly set up and was idle for the external trigger to start sampling. The recording software program acquired samples from the buffer in chunks of $100 \mathrm{~ms}(100$ $\mathrm{ms} \times 20$ samples per millisecond $\times 32$ channels $=64,000$ samples per multiplexed line). This $100-\mathrm{ms}(10-\mathrm{Hz})$ readout frequency offered a practical compromise between minimizing the time requirement introduced by the frequent updates of the processing code headers yet provided a smooth, real-time-like data display on the computer screen. After the buffer was filled, the acquired data were read out by the recording software and reordered to a $32 \times 2,000$ size matrix. The synchronous initiation of the sampling and clock advancement ensured that the data set of each buffer readout started with a sample corresponding to the first channel on the input side of the multiplexer chip (Fig. 1D). Although the actual clock code of the software and the hardware were not synchronized after the initiation, we never experienced any misalignment of the channel order even after extended testing of the entire system up to $40 \mathrm{kS} / \mathrm{s}$ sampling speed per channel. The electrophysiological recording setup was combined with a universal serial bus (USB) Web camera recording system for the continuous monitoring of the animal's position and behavior (Figs. 2 and 3).

Real-time hardware demultiplexing. Many applications need not only recording of the neuronal signal, but also its real-time availability, e.g., to interact with brain circuits in close-loop experiments (Berényi et al. 2012; Stark et al. 2012). However, the content of the buffer of the analog-digital card is read only once every $100 \mathrm{~ms}$, and the software routines introduced between an analog-digital and digital-analog conversion per se provide a suboptimal time precision for brain feedback stimulation. To achieve real-time readout ( $<50 \mu$ s per sample), we designed a mixed analog/digital demultiplexing device (Fig. 4). The demultiplexer, in addition to receiving analog data streams, was timed by the same clock signal that drove the headstages, and the rest of the clock bits were generated by the principle as described above for the multiplexers. Two separate bit-masks (2- and 3-bit) were generated by a microcontroller-driven user interface that allowed the selection of any one of the 8 multiplexed streams (using an analog switch circuit) and any of the desired channels of the selected stream (1 of 32). Demultiplexing was established by a pattern-matching algorithm: the real 5-bit clock signal was compared with the user-preset clock bit mask by a series of XNOR and AND logic gates. The digital output of the circuit generated a digital high level only if the clock mask matched the real clock signal (i.e., approximately the duration of the segment of the desired channel within the multiplexed stream). In principle, this digital pulse was suitable to trigger the sampling mode (i.e., a voltage follower) of a sample-and-hold integrated circuit, which worked as a voltage follower. The falling edge of the trigger pulse switched the sample-and-hold circuit to hold the last voltage value until the next sampling cycle, thus bridging the time gap, whereas the consecutive channels were transmitted in the multiplexer line (Fig. 4A). Because the propagation time of the digital gates introduced a significant delay in the digital trigger pulse, it could not be used directly to trigger the sample-and-hold circuit. To correct for the delay, a monostable multivibrator ("one shot") was used to generate a shorter pulse $(1.3 \mu \mathrm{s})$ so that the sampling was terminated before switching to the subsequent sample in the multiplexer line. The output of the sample-and-hold circuit was then low-pass filtered (at $10 \mathrm{kHz}$ ) to eliminate the

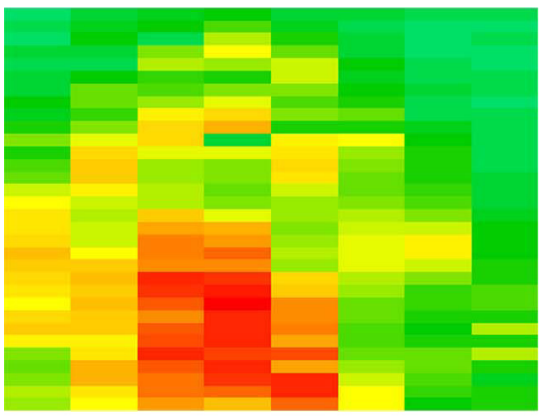

$90-140 \mathrm{~Hz}$

$\operatorname{Min}=118 \mu \mathrm{V} / \mathrm{V} \mathrm{Hz} ; \mathrm{Max}=690 \mu \mathrm{V} / \mathrm{VHz}$

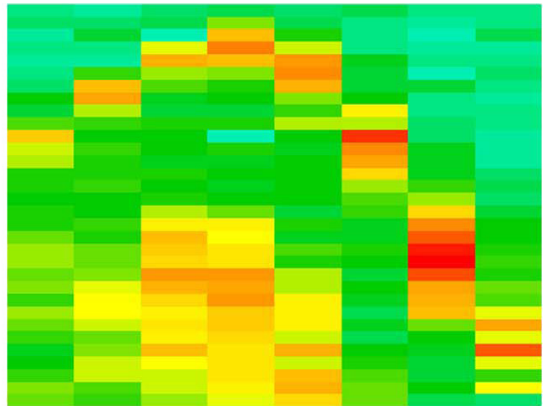

$140-300 \mathrm{~Hz}$

Min $=77 \mu \mathrm{V} / \sqrt{ } \mathrm{Hz} ; \mathrm{Max}=298 \mu \mathrm{V} / \mathrm{VHz}$

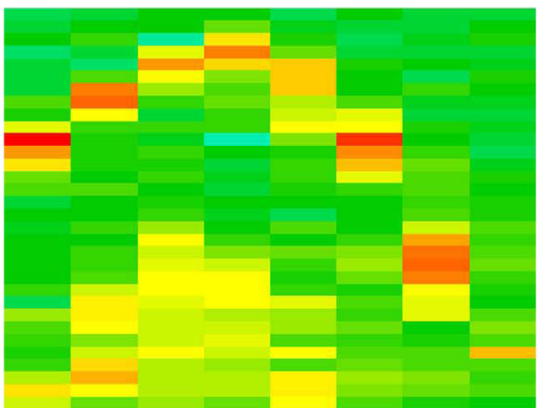

$300 \mathrm{~Hz}$

$\operatorname{Min}=44 \mu \mathrm{V} / \sqrt{ } \mathrm{Hz} ; \operatorname{Max}=187 \mu \mathrm{V} / \sqrt{\mathrm{Hz}}$

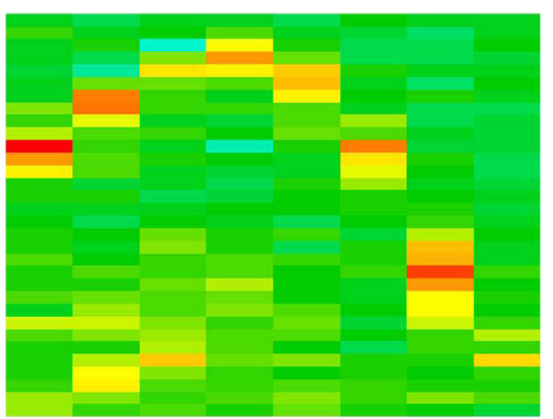

$300-600 \mathrm{~Hz}$

$\operatorname{Min}=32 \mu \mathrm{V} / \sqrt{ } \mathrm{Hz} ; \operatorname{Max}=122 \mu \mathrm{V} / \sqrt{\mathrm{Hz}}$
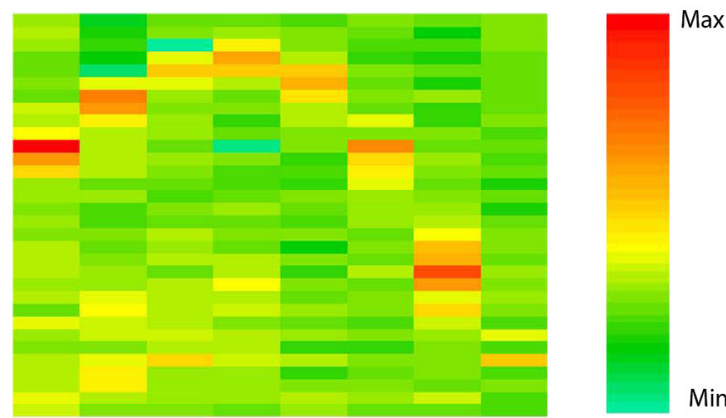

$300-6000 \mathrm{~Hz}$

$\operatorname{Min}=8 \mu \mathrm{V} / \sqrt{ } \mathrm{Hz} ; \operatorname{Max}=41 \mu \mathrm{V} / \sqrt{\mathrm{Hz}}$

Fig. 6. Layer-specific LFP power distribution of various frequency bands in the hippocampus. The arrangement of each panel is the same as Fig. 3A. Each panel is showing the power map of the same representative, 1-s-long recording segment containing sharp wave-ripples (SWR). For details on filtering, see MATERIALS AND METHODS. Min, minimum; Max, maximum. 
step responses (Fig. $4 B$ ). The reconstructed analog signal was amplified or filtered further as required for audio-monitoring the recorded signal or viewing the waveform on an oscilloscope $(0.5 \mathrm{~Hz}$ to $5 \mathrm{kHz}$; Fig. $4, D$ and $E)$.

Physical dimensions. To eliminate complex cabling and connections of high-channel counts of the probe, the probe was permanently connected to the 256-channel multiplexer headstage $(4.7 \mathrm{~g} ; 34 \times 39 \times 2.5 \mathrm{~mm})$ and is worn chronically by the rat. During recording, the animal was connected to the recording system through an ultraflexible cable (Vandecasteele et al. 2012; $12127-\mu \mathrm{m}$-diameter insulated copper wires, 455$\mu \mathrm{m}$-total-diameter cable; Fig. $2 F$ ). For recording from smallersized silicon probes or tetrodes targeting multiple brain structures, we constructed 32- and 64-channel versions of the multiplexer headstage (32-channel: $0.9 \mathrm{~g} ; 13 \times 15 \times 3 \mathrm{~mm}$; 64-channel: $1.2 \mathrm{~g} ; 13 \times 19 \times 3 \mathrm{~mm}$, respectively; Fig. 3). In contrast to the 256-channel version, the front ends of these headstages were connected to the probe output by high-density connectors (Omnetics) during the experiments.

Validation of the recording system and estimation of the signal quality. We estimated the mean input referred noise spectrum for all channels of the 32-channel multiplexer headstage (the results presented here apply to the 64- and 256channel headstages as well since they consist of parallelconnected 32-channel blocks). When the input channels were short-circuited with the reference pin of the headstage, the mean root-mean-square amplitude of $60-\mathrm{Hz}$ noise was $7.2 \mu \mathrm{V}$ (range $=2-8.5 \mu \mathrm{V})$, which is approximately one order of magnitude higher than the half least significant bit of the A/D conversion. We also compared the signal quality with a commercially available recording system (HST/32V-G20 preamplifier; Plexon) and Digital Lynx 16SX A/D converter (NeuraLynx). The amplitude of the signal was $\sim 20 \%$ smaller using the Intan amplifier chips than that of the nonmultiplexing control system, which can be explained by the lower input impedance of these chips. However, the signal waveforms and the wave shapes of the extracellular spikes were virtually identical. The mean difference between the normalized spike waveforms of a well-isolated test single unit, recorded by the current and the control system, was $10 \%$ of the standard deviation of the waveform recorded by the control system. The common noise component across channels in the recorded LFPs was significantly lower, especially in the lower frequency ranges, which is presumably a result of the serial transmission of the channels in the multiplexed line instead of the nonmultiplexed parallel transmission. The intrashank correlation of the recorded signals (i.e., the mean of the correlation values calculated between pairs located on the same shanks; see MATERIALS AND METHODS) was similar in both systems for every investigated frequency range. However, the mean correlation across the shanks gradually decreased at higher frequencies in our system $(r=0.8,0.72$, and 0.23 for $>1,>10$, and $>100$ $\mathrm{Hz}$, respectively) while slightly increased for the control system $(r=0.55,0.72$, and 0.75$)$. The single-unit yield was larger in our system despite the slightly lower signal amplitude and lower sampling rate (20 vs. $34 \mathrm{kS} / \mathrm{s})$. After the automatic spike

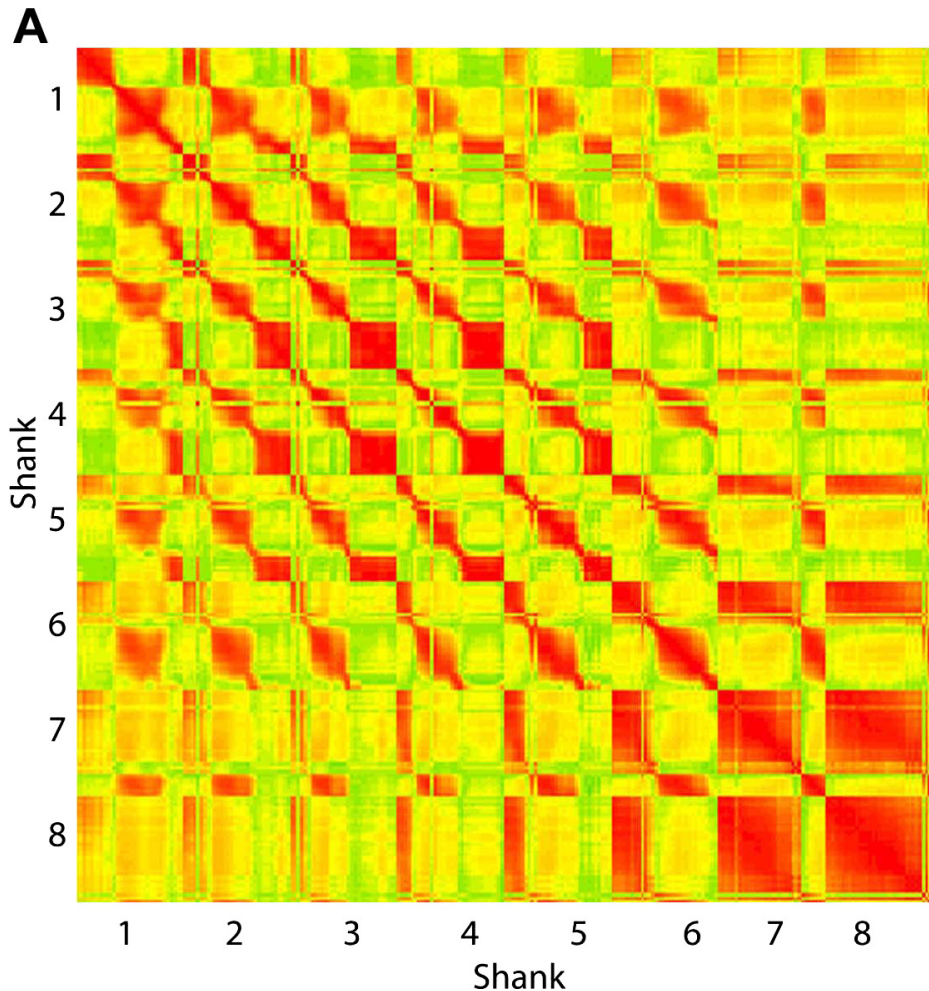

B
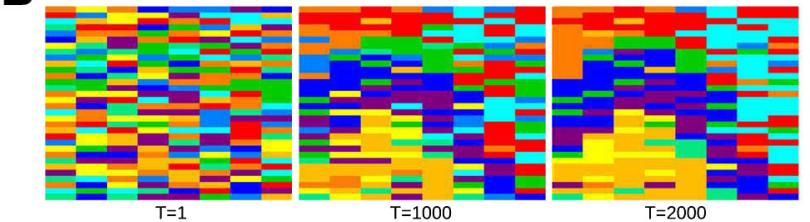

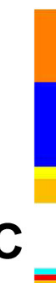

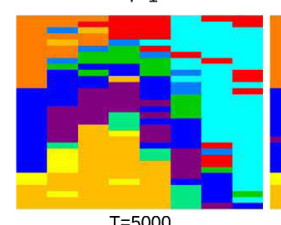
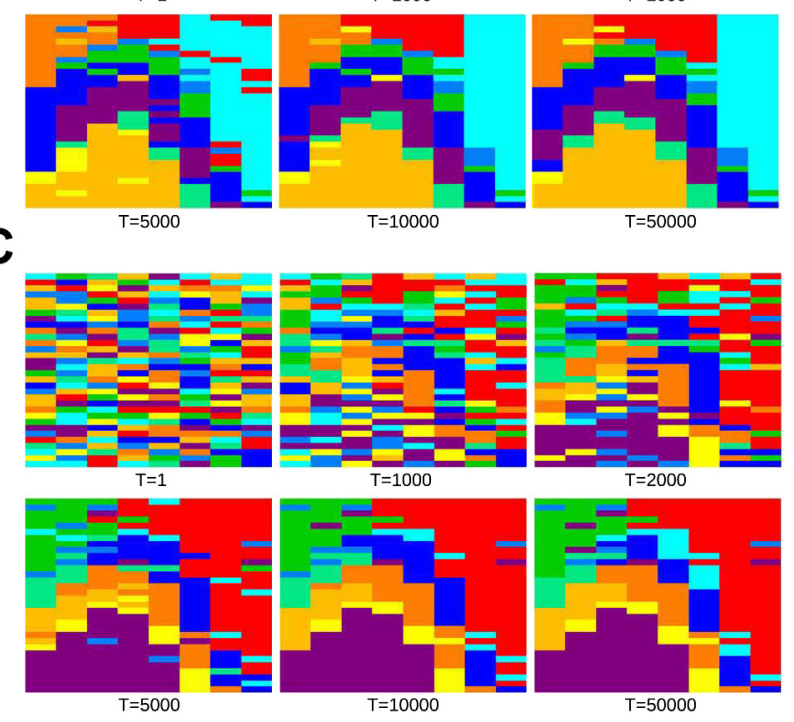

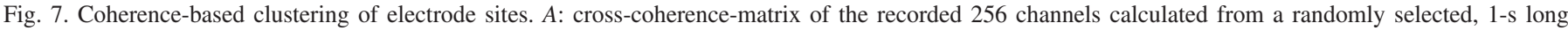

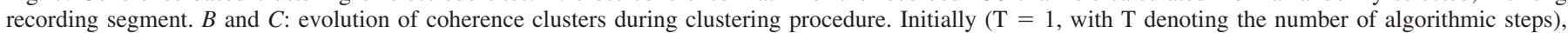

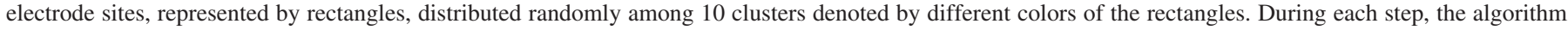

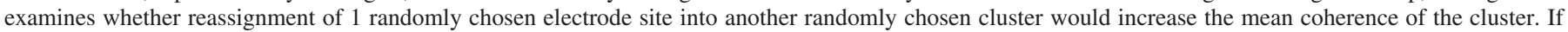

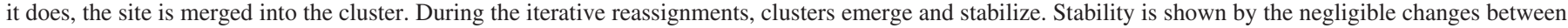

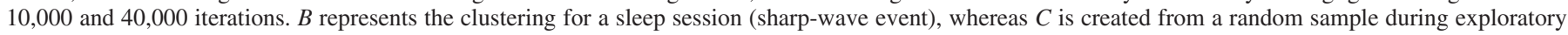
behavior (theta). 
extraction and clustering of the sample session recorded by both systems (see MATERIALS AND METHODS), 26 and 7 single units were detected on a single shank using our (test) and the control systems, respectively. After taking account of cluster quality criteria, nine vs. five clusters, respectively, satisfied our conservative criteria (see MATERIALS AND METHODS).

\section{Physiological Recordings}

Electroanatomy of cortical layers. We have implanted the high-density, 256-site probes in 5 rats. In 2 rats, 2 probes were implanted (512 channels). In 1 rat the probes were placed in the same hemisphere, whereas in the other animal they were placed symmetrically in the 2 hemispheres (Fig. 2).

The relationship between laminar arrangement of afferents and the characteristic depth profiles of various oscillatory and irregular LFP patterns can offer means for an identification of the various cortical layers and their transitions (Buzsáki et al. 2012; Montgomery et al. 2008). Here, we illustrate our strategy in the hippocampus, for which laminar structures and wellunderstood LFP patterns allow for an online quantitative identification of each of the recording electrode sites (Montgomery et al. 2008).

First, each of the 256 LFP signals was filtered by a narrow Gaussian band-pass filter (peak $=300 \mathrm{~Hz}, \mathrm{SD}=10 \mathrm{~Hz}$ ), and power values, determined from a randomly sampled 1 -s epoch, are displayed for each of the 256 sites as a 2-D map (Fig. 5A). This frequency band was empirically determined by selecting a frequency band with the largest ratio of power between cell body layers and dendritic layers (Fig. 6). The band we selected (300-Hz Gaussian filter, SD $=10 \mathrm{~Hz}$ ) presumably represents various aspects of MUA (Ray and Maunsell 2011) since its spatial distribution clearly marked the pyramidal layer and the dentate-hilar area, as reflected by the density of the recorded units. The soma location of the recorded neurons was estimated by the largest amplitude waveform representing the firing of each unit (Csicsvari et al. 2003; Fujisawa et al. 2008) and assigned to the recording site of the relevant shank in the electroanatomic map (Fig. 5A). In the next step, coherence maps in the gamma frequency band (40-90 Hz) were constructed. Each site served as a reference, and coherence distribution with each of the remaining 255 sites was determined iteratively (Montgomery et al. 2008; Figs. 5B and 7A). Using a gradient-descent algorithm (Fig. 7), the recording sites were clustered based on the resulting coherence matrix, and the resulting coherent channels were grouped together. Next, a 2-D gamma coherence map was generated (based on the spatial locations of the recording sites and their coherence cluster identities) and combined with the power map (Fig. 5C) to identify the cellular layer among the emerging clusters. The boundaries of the channel clusters happened to be nicely overlapping with the anatomic layers without any a priori knowledge of the histological control. In a different display, each recording site was connected to its most coherent neighbor on the adjacent shanks. The connecting lines were colorcoded on the basis of cluster identity of their leftmost member site. The resulting coastline display provided a smooth, layerspecific map of the recorded space by the silicon probe (Fig. $5 C$, right). The electroanatomic map constructed from unit firing and LFP signals corresponded faithfully to the histological reconstruction of the electrode tracks and the anatomic layers of the hippocampus (Fig. 5D).

High-density silicon probe recordings in the neocortex were similarly advantageous for cortical layer identification. Gamma coherence reliably identified three separate layers, corresponding to the superficial (II/III), middle (IV), and deep (V/VI) layers (Fig. 8A).

Large-scale recording of unit activity. Figure $8 B$ shows wide-band $(0.5 \mathrm{~Hz}$ to $10 \mathrm{kHz})$ traces from a single shank in the somatosensory cortex. Neurons were recorded from all shanks of the probes in both hemispheres for several days before advancing the probes into the hippocampus. In conjunction with LFP, population patterns of unit firing allowed for a clear classification of brain states (Fig. 8C).
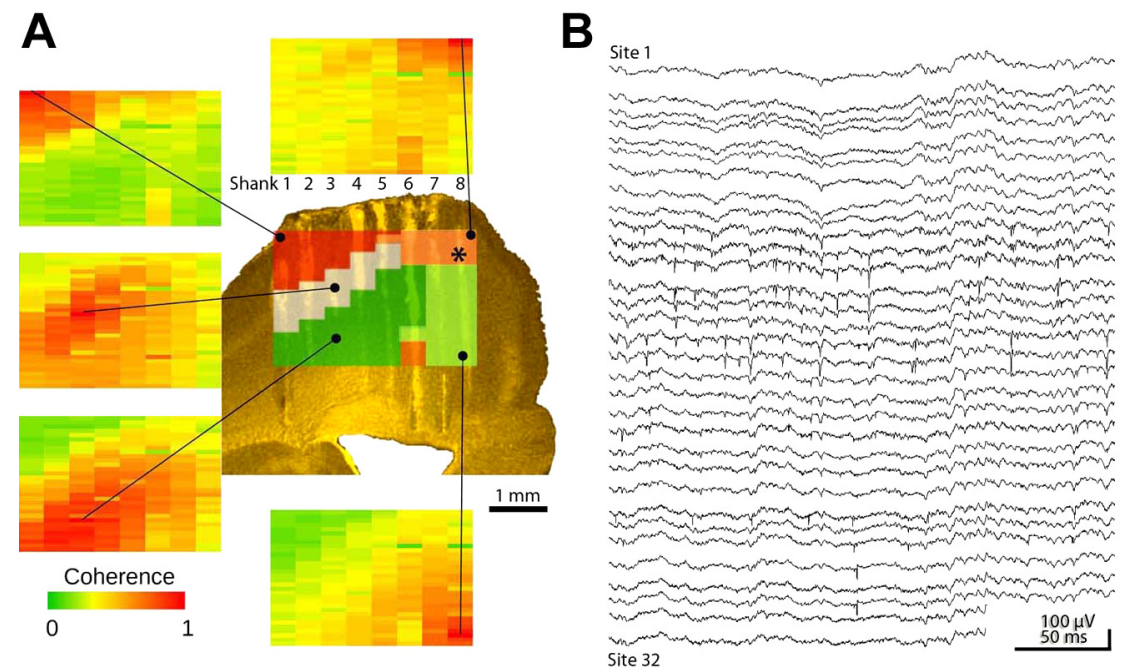

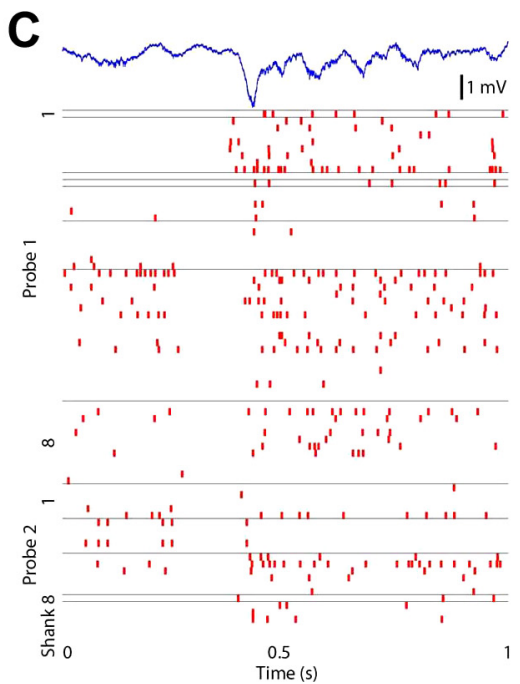

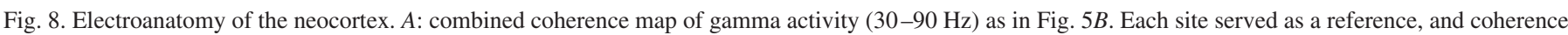

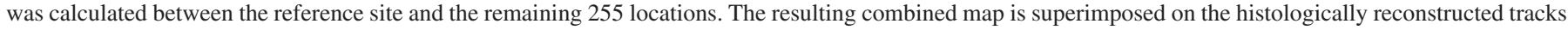

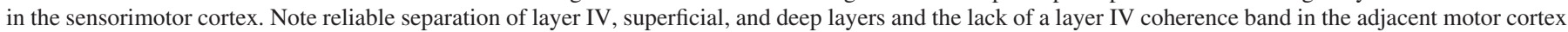

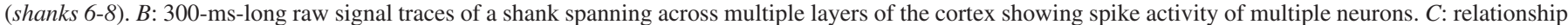

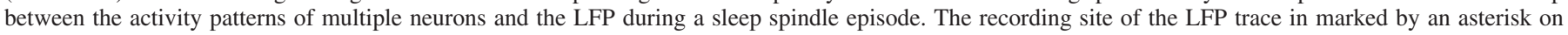
the top of the panel in $A$. The figure is a representative sample for illustration purposes only. 
Neocortical and hippocampal units were clustered semiautomatically (Harris et al. 2000) followed by physiological classification of the units into putative principal cells and interneurons (Barthó et al. 2004; Fujisawa et al. 2008). To obtain "at glance" information about the recorded neurons, an algorithm was designed to summarize the main physiological and behavioral correlates of each unit (Fig. 9). First, the mean waveform of the unit recorded at multiple sites is displayed together with wave-shape classification of the unit and its autocorrelogram and ISI histogram. The next level of analysis characterizes the spike-LFP relationships. In case of hippocampal neurons, the preferred firing phase in relation to theta, gamma, and ripple oscillations of the unit are displayed together with sharpwave-related firing (Fig. 9). The third level of analysis shows the behavioral correlates of the neuron such as its place-related firing in the maze and spike-phase precession relative to the theta rhythm (Fig. 9). The display panels can be flexibly replaced with other analyses such as displaying the anatomic location of the neuron in 2-D space, its cluster space relative to surrounding clusters, brain-state dependence of firing rates, and cross-correlation with selected other neurons.

The 256-site probe was designed for large spatial coverage to allow monitoring the activity in multiple hippocampal layers. This inevitably compromised the density of the recording sites $(50 \mu \mathrm{m})$ and, consequently, reduced the unit clusters with high isolation quality (MATERIALS AND METHODS) compared with higher-density (20- $\mu \mathrm{m}$ spacing) probes. However, the lower unit yield per site was offset by the ability of the 256-site probe to record from multiple layers (Fig. 10).
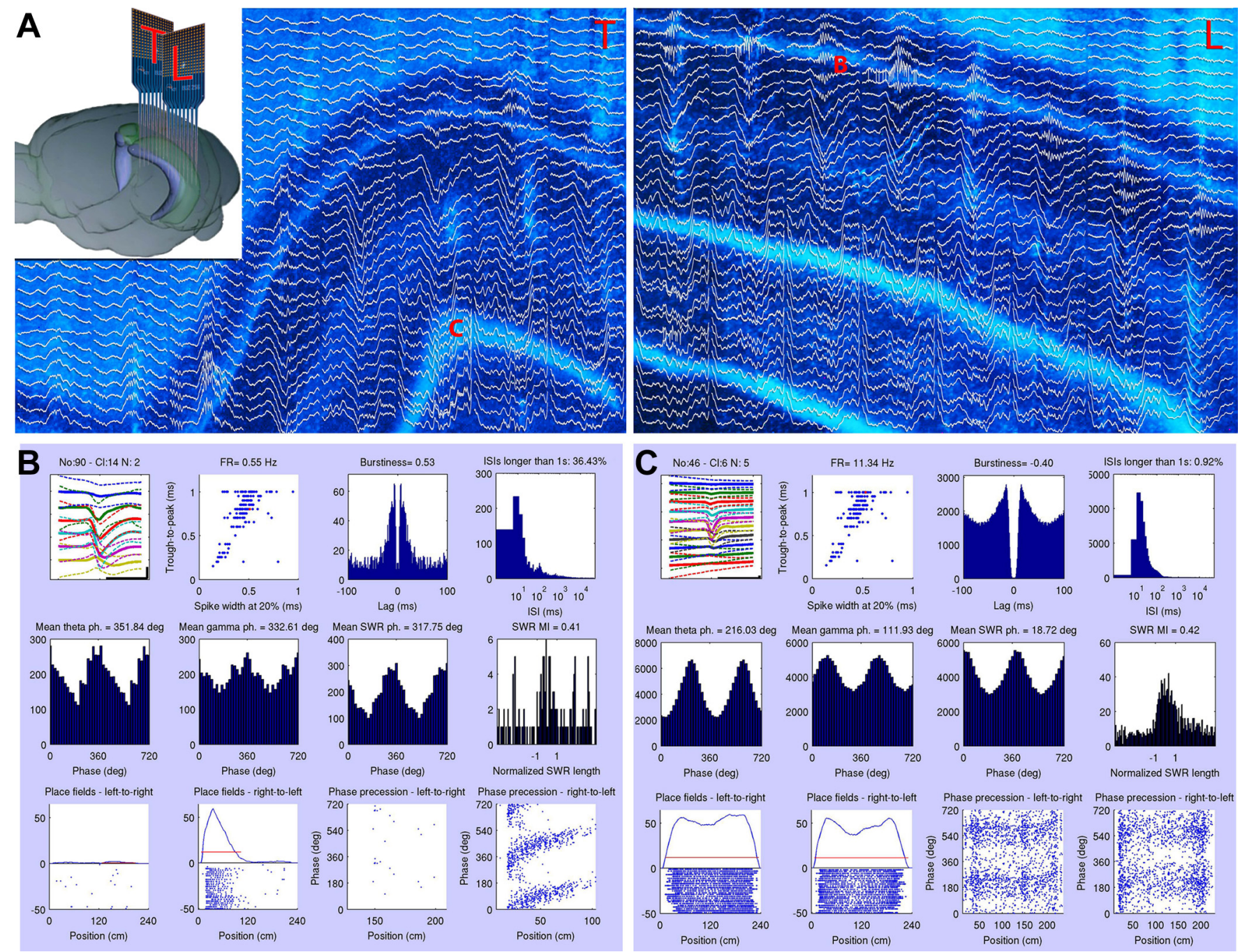

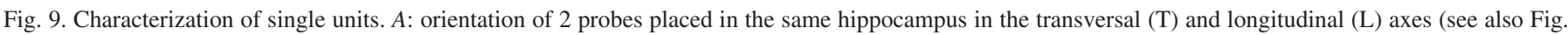

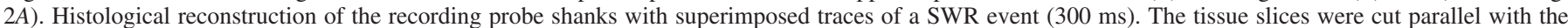

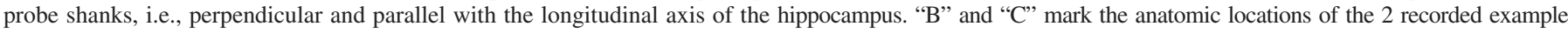

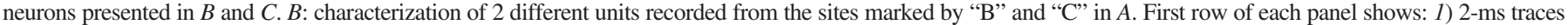

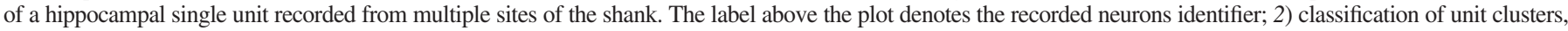

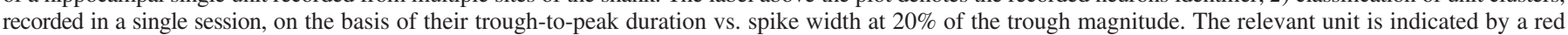

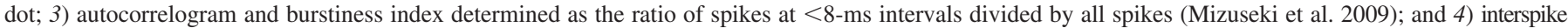

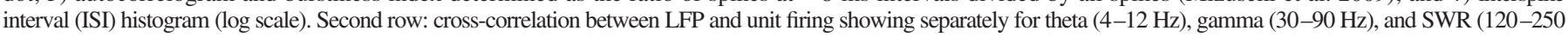

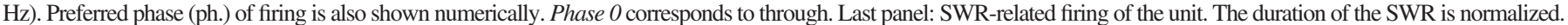

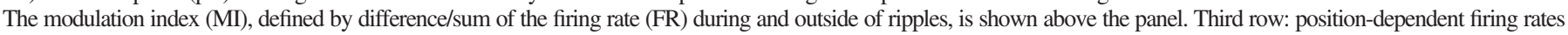

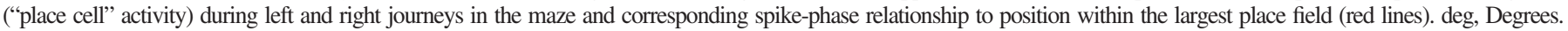


Large-scale recording from local circuits has the power of identifying monosynaptic connections at least between principal cells and interneurons (Fujisawa et al. 2008). This is typically done by examining counts of cooccurrences of spiking in the putative pre- and postsynaptic neurons as exemplified by the large peaks at short-latency time lags (Fig. 11, $A$ and $B$ ). Our analysis is limited only to pyramidal-interneuron interactions since the validation of the monosynaptic peaks on the cross-correlograms of the pyramidal-pyramidal neuron pairs was not reliable due to their low firing rates. However, with extended recordings and increased number of spikes, the circuit analysis can be extended to pyramidal-pyramidal connections as well (Hirabayashi et al. 2013). Of the 26,406 possible connections of a total of 163 simultaneously recorded neurons (counting each literal pair twice, corresponding to the 2 directions) recorded by 2 probes (512 sites) in the same hippocampus (Fig. 11, $A$ and $B), 137$ pairs $(0.005 \%$; Fig. $11 C$ ) had short latency $(<5$-ms onset) and narrow significant peaks $(<2 \mathrm{~ms})$ or troughs in their cross-correlograms, indicating that the presynaptic partner neuron was an excitatory or inhibitory neuron, respectively. In most cases, the postsynaptic targets were interneurons, as judged by their high firing rates and waveforms (Sirota et al. 2008). Most functionally connected pairs were detected locally in the same hippocampal region, but several connections were detected between neurons in the CA3 and CA1 regions as well (Fig. 11). Several pyramidal cells of both local and distant origin could converge on the same putative interneuron.

Increasingly larger numbers of simultaneously recorded neurons also facilitate the search for spatiotemporal patterns of neuron interactions in local circuits because the likelihood of finding connected pairs increases quadratically with the number of the recorded cells (Barthó et al. 2004; Carandini 2012). Since the spike transmission probability (i.e., the excess numbers of postsynaptic spikes divided by the number of presynaptic spikes, reflecting the efficacy of spike transfer function) can be used as an indirect measure of synaptic strengths between neurons (Fujisawa et al. 2008), the magnitude of spike transfer can be used in future studies to estimate the state- and task-related circuit reconfigurations (Fujisawa et al. 2008).

State-dependent activation of single neurons by multiple inputs. In the intact brain, neurons are embedded in interconnected networks and respond with spikes to a single input or a combination of inputs. Since afferents to hippocampal neurons target specific layers, high-density recordings from multiple layers and regions can, in principle, track the activity of the afferents giving rise to each spike. To illustrate the principle, we sampled LFP multiple times at all recording sites in 20-ms windows, centered on the spikes of a CA1 pyramidal neuron during sharp wave-ripple events and ambulation while the rat was walking through the place field of the neuron (O'Keefe and Nadel 1978; Fig. 12; Supplemental Video S1, available in the data supplement online at the Journal of Neurophysiology
Fig. 10. Comparison of single-unit isolation metrics. Each measure is shown for 6 different recording conditions (probe, structure, and species). $A$ and $C$ : Mahalanobis distance of isolated spike clusters in high-dimensional feature space. $B$ and $D$ : contamination of isolated single-unit clusters calculated as the ratio of spikes occurring within $(2 \mathrm{~ms})$ and after $(20 \mathrm{~ms})$ the refractory period of the given neuron. Medians and the interquartile ranges are shown. $\mathrm{N}$ denotes the number of neurons fulfilling the inclusion criteria. $A-D$ display the same data set using permissive and conservative inclusion criteria, respectively. ID, isolation distance; Hip, hippocampus; $\mathrm{Cx}$, cortex; $2 \times 256,2$ 256-channel silicon probes with multiplexing headstages; 64, Buz64-type silicon probe with $20-\mu \mathrm{m}$ intersite distance with multiplexing headstage; 64Sp, Buz64Sp-type, 6-shank silicon probe with $20-\mu \mathrm{m}$ intersite distance equipped with an optical fiber on each shank recorded by a nonmultiplexing headstage and amplifier.

Permissive (ID>30 or ISlindex<0.4):

A

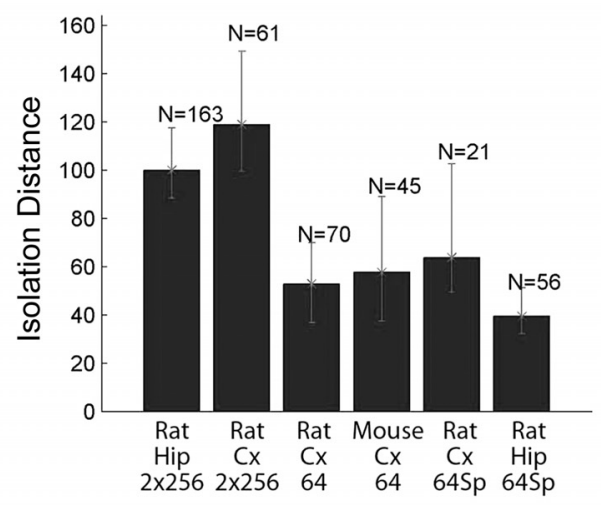

Conservative (ID $>40$ and ISlindex $<0.2)$ :

C

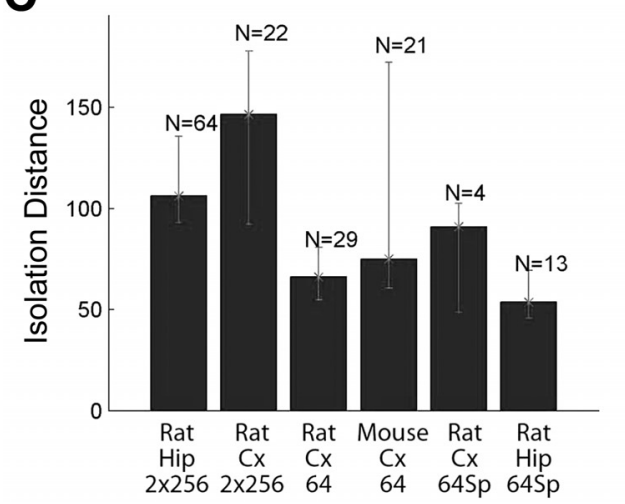

B
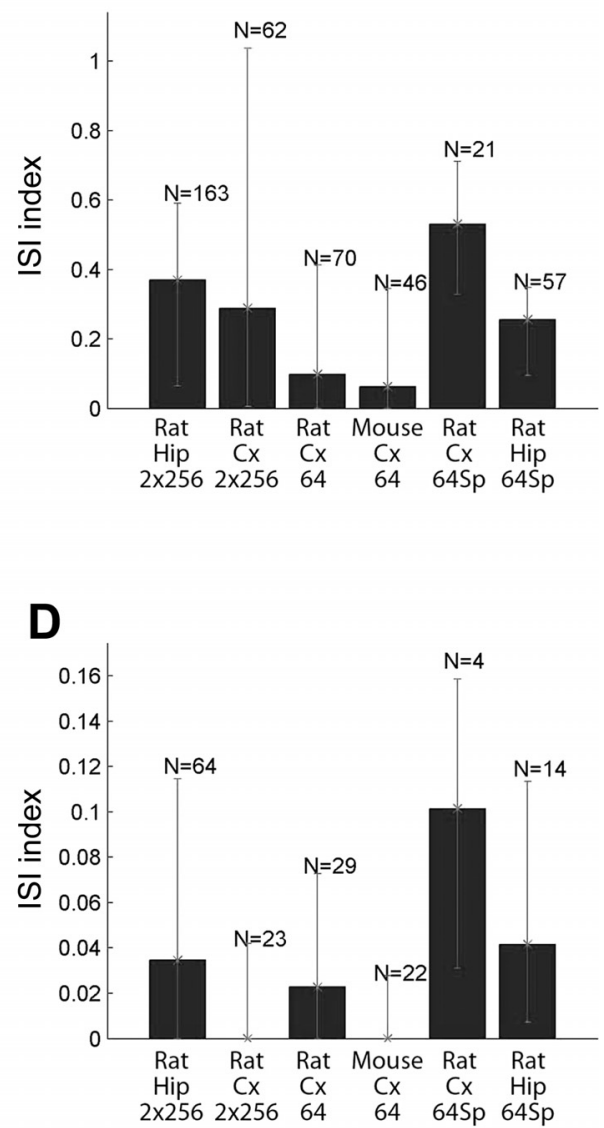

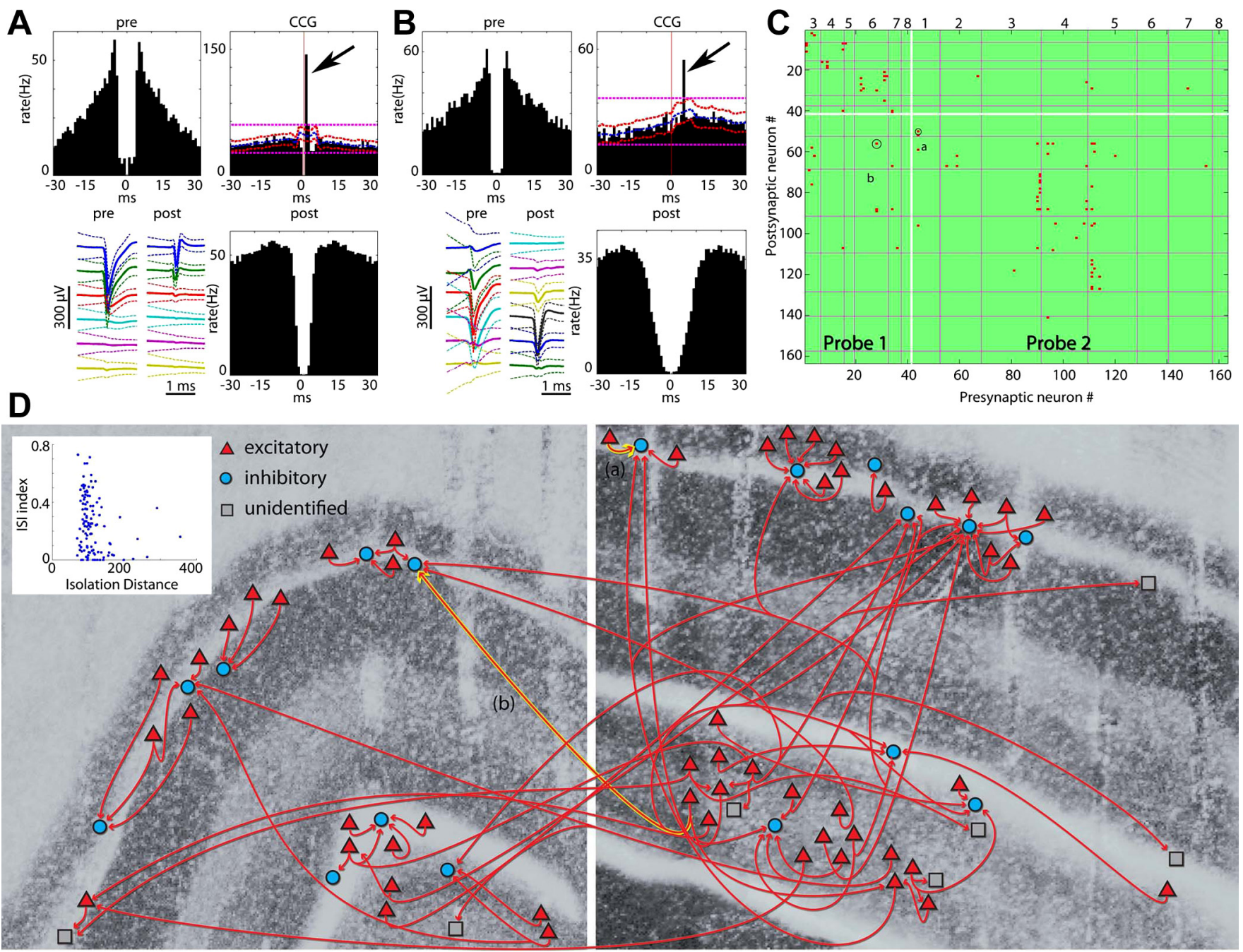

Fig. 11. Partial circuit reconstruction from physiological interactions. A: identification of monosynaptic connections. Only pyramidal-interneuron connections are shown. Autocorrelogram of the reference (presynaptic) neuron (pre), referred (postsynaptic) neuron (post), and cross-correlogram (CCG) between the neuron pair. Short-latency $(<1 \mathrm{~ms})$ narrow peak (arrow) identifies the reference cell as a putative excitatory (pyramidal) neuron. Blue line, mean of time-jittered spikes; red line, pointwise comparison $(P<0.01)$; magenta line, global comparison $(P<0.01$; for explanation, see MATERIALS AND METHODS; Fujisawa et al. 2008). $B$ : same as in $A$ from another pair with members recorded from the CA3 and CA1 regions. $C$ : CCG matrix based on 26,406 simultaneously recorded neuron pairs $(n=$ 163 neurons) in a single session. Red pixel: monosynaptic connection (based on significant short-latency peaks) with reference neuron as putative pyramidal cell $(n=127)$. White lines: separation of neurons recorded by probe 1 and probe 2 . Numbers identify the recording shanks. CCG shown in $A$ and $B$ are circled. $D$ : circuit diagram reconstructed from monosynaptic connections (for shank orientation, see Fig. 2). Red triangles: excitatory neurons. Blue circles: putative inhibitory interneurons. Gray squares: unidentified neurons. Local and CA3-CA1 connections, marked with (a) and (b) (as shown in $A$ and $B$ ), are highlighted by yellow. Note convergence of multiple pyramidal cells on target interneurons. The figure is a representative sample for illustration purposes only.

web site). Sharp wave-ripples are self-organized population patterns that arise in the $\mathrm{CA} 3$ region and depolarize the midapical dendrites of CA1 neurons as seen extracellularly by a large-amplitude negative wave in stratum radiatum (Ylinen et al. 1995). As expected, LFP activity before the occurrence of spikes during sharp wave-ripple events was most prominent in the CA1 s. radiatum (Fig. 12A; Supplemental Video S1). In contrast, during place-related activity, pyramidal cells are believed to be discharged by a combination of the direct entorhinal input terminating in s. lacunosum-moleculare and the CA3 input (Moser et al. 2008; Fig. 12B). During ambulation, LFP activity before spike occurrence was first observed in the s. lacunosum-moleculare and dentate molecular layer followed by the CA3 activation in CA1 s. radiatum before the spikes of the place cell. These findings illustrate the exceptional power of the combination of recording spikes and high spatial density monitoring of LFP for understanding the input-output transformation of single neurons.

Large-scale recordings from the mouse brain. Multiplexing neuronal signals is even more critical for recordings from mice because the size and weight of the preamplifiers and the connecting cable can seriously affect the behavioral performance of the animal. Either 32- or 64-channel headstages and their combinations are deployed for high-density recordings from 2 or 3 brain structures, including various neocortical areas, hippocampus, thalamus, nucleus accumbens, and ventral tegmental area. In addition to the multiplexed outputs, the recording headstage contains 2 detachable light-emitting diodes (LEDs) for tracking the animal's position with a video camera and a 3-dimensional accelerometer to monitor fast head movements. Figure $13 \mathrm{~A}$ shows illustrative neocortical traces from a mouse exploring an open maze. The silicon probe 


\section{A sWR}
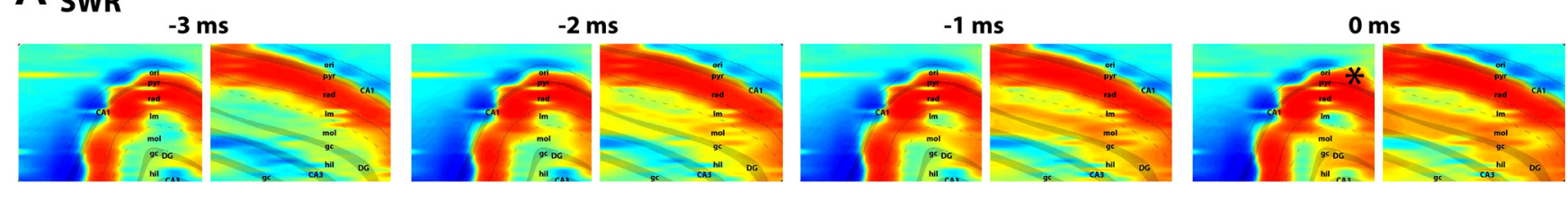

\section{B Theta}
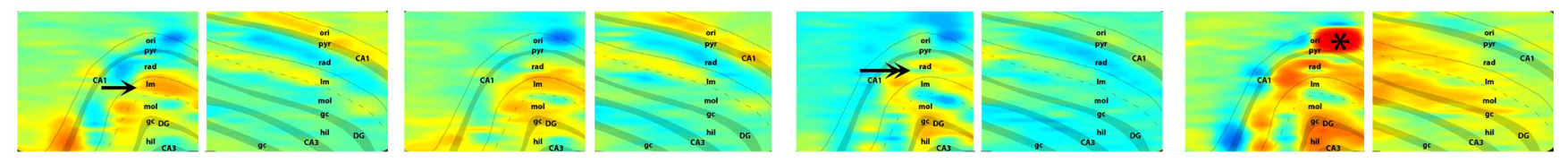

Fig. 12. Spikes are embedded in unique and spatially distributed LFP. Spike-triggered averages of the LFP in the hippocampus during slow-wave sleep (A) and exploration $(B)$. During sleep, spikes were sampled during SWR; during exploration (theta), spikes of the same neuron were sampled while the rat ran on a linear track for a water reward. Recordings were made by 28 -shank (300- $\mu \mathrm{m}$ intershank distance), 256-site silicon probes. The LFP was smoothed and interpolated both within and across shanks. The LFP was triggered by the spikes of a pyramidal neuron in CA1 pyramidal layer (pyr; shown by an asterisk). Both frame sequences show $450-\mu \mathrm{s}$-resolution snapshots of the LFP map before ( -3 to $-1 \mathrm{~ms})$ and at the time of the spike occurrence $(0 \mathrm{~ms})$. The 2 images of each frame are showing the activities on the 2 probes (as shown in Fig. 9). Note that during sleep (A), activity arises (negative wave, hot colors) in CA3 and invades the CA1 stratum radiatum $(\mathrm{rad})$. During exploration $(B)$, the spike is associated with synaptic activity mainly in the s. lacunosum-moleculare (lm; shown by an arrow) followed by the radiatum layer (double arrow), indicating a combination of entorhinal cortex and CA3 input activation. The LFP map changes characteristically with time (see Supplemental Video S1). ori, Oriens layer; gc, granule cell layer; hil, hilus.

recordings are most often combined with optogenetic manipulation of the recorded neurons, using laser or LED-powered sharpened optical fibers that deliver light to the tips of the probe shanks (Berényi et al. 2012; Royer et al. 2010; Stark et al. 2012; Fig. 13B).

\section{DISCUSSION}

We have described a complete system that allows highchannel-count recordings from a small volume of neuronal tissue using a lightweight signal multiplexing headstage that permits free behavior such as exploration in mazes. This was achieved by multiple stages of development, including multishank high-density recording silicon probes, ultraflexible interconnect, miniaturized microdrive (Vandecasteele et al. 2012), and, most importantly, high-throughput on-stage signal multiplexing, remote-site digitization, and demultiplexing, which allow to link the animal to the recording/processing hardware by an ultralight cable. The system has been extensively tested for large-scale recordings of spike outputs and LFP signals in both rats and mice. The 2-D distribution of the recording sites distributed across several layers and regions can provide important information about the distribution of LFP together with large-scale unit recordings.

Sensing of electrical activity is complementary to opticsbased imaging methods (Alivisatos et al. 2013). Silicon probes can access deep brain structures and offer sampling speed at the level of neuronal communication. They provide neuronscale resolution in local circuits and permit the study of interactions of multiple brain regions, currently not practical with imaging methods (Ghosh et al. 2011). Electrodes are invasive, a feature that can be improved with further size reduction, refinement of tip configuration, and tissue-compatible coating ( Du et al. 2011; Kipke et al. 2008; Wise et al. 2004). In the current configuration, the combined volume of the 8 recording shanks of the 256 -site probe is comparable with the volume of 8 wire tetrodes (Wilson and McNaughton 1993) but can record from significantly larger numbers of units in addition to providing high-spatial-resolution LFP signals. Even the 8-shank, 64-site probe can record from $>100$ neurons (Fujisawa et al. 2008). In addition, linear probes have the advantage of online identification of the recording layers, the spatial localization of the main current sources and sinks, and the positions of the recorded neurons (Csicsvari et al. 2003). We present here a method to determine the position of the electrodes during experiments, exploiting the high within-layer gamma band coherence of the LFP. Polarity reversal of sleep spindles, K-complexes, and other LFP patterns can also be used to increase the precision of layer segregation. Since the layer identification we described is done in situ, it allows for the determination of the recordings sites in each session. This is a major advantage in experiments when the probe is moved gradually in the course of multiple sessions and for layerrestricted optical stimulation in future experiments.

The 50- $\mu \mathrm{m}$ spacing of the recording sites on the 256-site probe (Figs. $1 A$ and 10 ) is suboptimal for unit recordings and cell clustering (Harris et al. 2000). Denser recording sites without increasing the shank volume is desirable. On the other hand, the additional 2-D recording of the LFP from multiple layers and regions is an additional benefit. Because of the rapid development of silicon technology, probes with $>1,000$ site counts and $20-\mu \mathrm{m}$ site spacing, yet without further size increase, are expected in the near future. Ultimately, a dynamically reshapeable probe design (by selecting recording sites within the cellular layers with high-quality unit activity) would overcome the tradeoff of the current probe design by significantly improving the single-unit yield and still keeping the amount of recorded data reasonable.

In addition to significantly increasing the number of recording sites, the size of the multiplexers should also be considerably reduced. Although the size and weight of the extracranial devices is less of a problem in head-fixed animals, currently they are the most limiting factors in experiments that require free movement of small rodents. In the current configuration, silicon probes are connected to the multiplexers via an ultraflexible polyimide cable, but the multiplexers have to be manually soldered onto the printed circuit board. In addition to size 
Innovative Methodology

512-CHANNEL NEURONAL RECORDING IN BEHAVING RODENTS

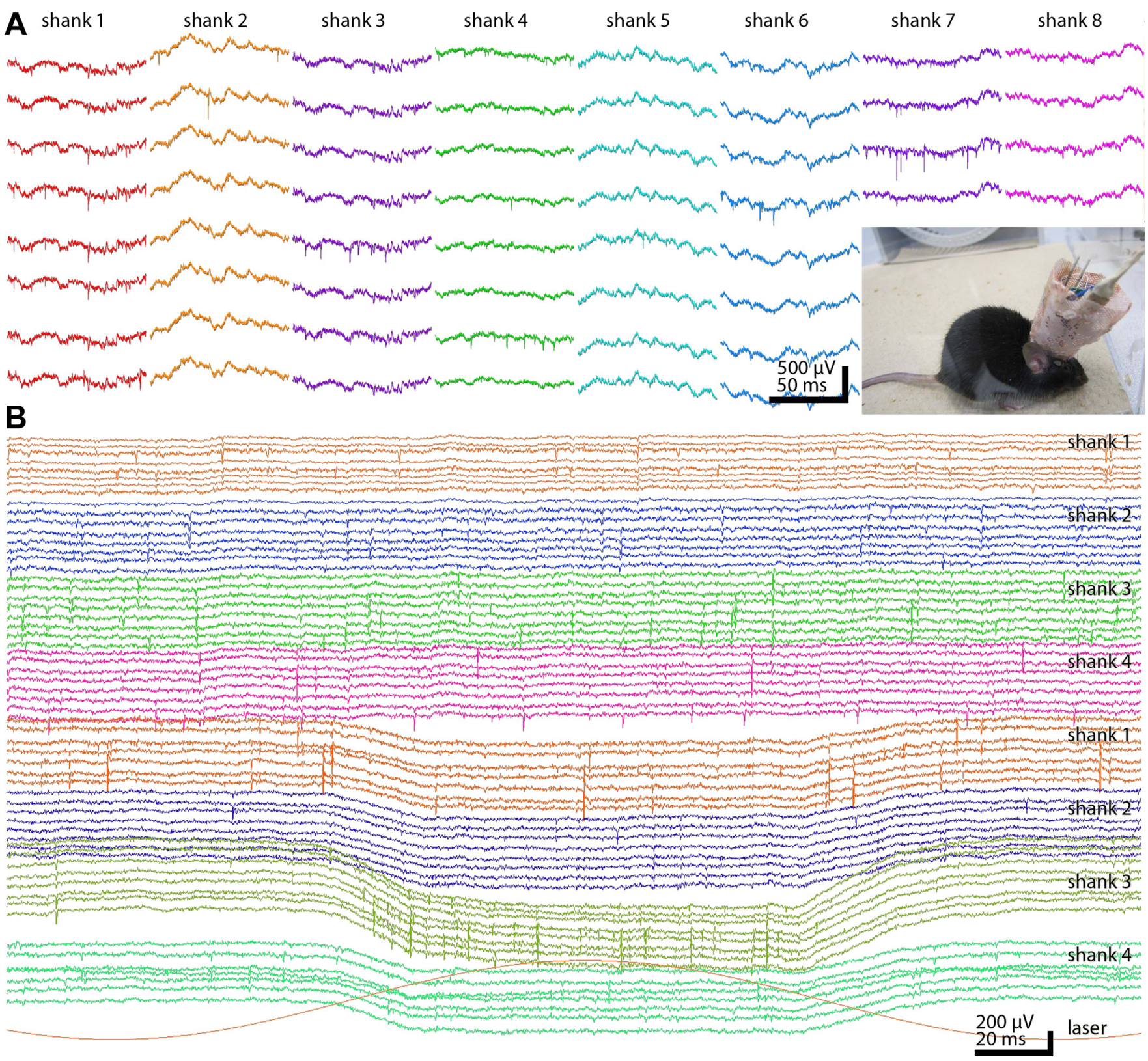

Fig. 13. Unit and LFP recordings from the mouse. A: chronic recordings from a mouse using an 8-shank 64-site silicon probe. One hundred-millisecond epochs from each shank are shown. Inset: headstage with silicon probe, microdrive, and 64-channel signal multiplexer surrounded by copper mesh shielding. The freely moving mouse is connected to the equipment by an ultraflexible cable. $B: 24$-shank, 32 -site probes were placed in the nucleus accumbens (top shanks 1-4) and ventral tegmental area (VTA; bottom shanks 1-4) in a TH-Cre;Ai32 mouse, expressing ChR2 in tyrosine hydroxylaseexpressing neurons. One of the shanks in the VTA also contained an optical fiber for light delivery (Stark et al. 2012). Note VTA neuronal responses to 472-nm (bottom red trace) laser light stimulation.

limitation, the numerous mechanical connections among the probe, interconnect, multiplexers, and the offsite demultiplexer increase failure rate.

In terms of the cluster quality of units, our system outperformed a benchmark commercial recording system. The relatively low input impedance of the Intan RHA-2132 multiplexer chip (13 $\mathrm{M} \Omega$ at 1 $\mathrm{kHz})$ and its relatively high-input capacitance $(12 \mathrm{pF})$ attenuated the recorded signal by $\sim 20 \%$, comparing with another nonmultiplexed headstage. However, the serial, temporally noncoincident transmission of the multiple channel segments makes the multiplexer more resistant to mechanical cable artifacts. Signal attenuation of the multiplexer could be eliminated by adding buffer operational ampli- fiers before multiplexing. However, such modification may double the current size of the multiplexer, adding unwanted additional weight and volume to the headstage.

New generation probes may have on-probe signal amplification and multiplexing capabilities (Csicsvari et al. 2003; Olsson et al. 2005). Alternatively, the required signal multiplexing circuits can become an integral part of the flexible interconnect cable (Viventi et al. 2011). The use of flexible and active interface electronics may offer an alternative approach that may be advantageous for several applications. The digital output of the multiplexers (Harrison 2008) allows direct streaming of the neurophysiological data to the computer 
without the extra step of A/D conversion. Digital multiplexers also offer noise reduction since potential noise sources of cable transmission, further amplification, and offsite digital conversion can be eliminated (Harrison 2008). In principle, amplification and multiplexing can be placed on the probe shanks, effectively reducing the required large numbers of interconnects between the recording sites and electronics outside of the brain. Unfortunately, current CMOS circuits require $\sim 1-\mathrm{V}$ supply voltage and may generate both excess heat and local electric fields that may modify the activity of nearby neurons if placed on the shank itself (Berényi et al. 2012). Thus, without dramatic reduction of the power consumption, brain-embedded electronics remain a major challenge (Alivisatos et al. 2013).

In principle, cables interconnecting the headstage and the recording system can be completely eliminated by telemetry. Although up to 64-channel telemetry systems have been successfully used in small animals (Greenwald et al. 2011; Harrison et al. 2011; Sodagar et al. 2007; Szuts et al. 2011) and may be the only solution for specific applications (Yartsev and Ulanovsky 2013), multiplexing and ultraflexible cables allow much higher bandwidth, higher channel counts, and lower noise. The power source required for telemetry adds weight and limits the duration of the experiments, especially in mice.

Computation in brain circuits is performed by numerous specialized neurons. Identification and manipulation of specific neuron types in the behaving animal has recently become possible with optogenetics (Boyden et al. 2005) combined with ever-increasing specificity of neuron labeling, mostly in mice (Madisen et al. 2012; Taniguchi et al. 2011). Therefore, perhaps the most important new direction should be the development of combined techniques that allow precise delivery of light locally to the simultaneously recorded neurons. Experiments have shown that as low as $5-15 \mu \mathrm{W}$ of light is sufficient to activate ChR2-expressing neurons in vivo (Stark et al. 2012). However, construction and use of silicon probe devices with etched optical fibers that allow precise local delivery of light energy are currently done manually, and only few laboratories have the capability to use such methods routinely (Anikeeva et al. 2012; Royer et al. 2010, 2012; Stark et al. 2012). To bridge the gap between optogenetics and large-scale recording of neurons, there are at least two possible viable solutions. The first one is adding optical waveguides integrated into the shanks of the silicon probe and coupling the back end of the waveguides to lasers, LEDs, or laser diodes (Stark et al. 2012; Wu et al. 2013; Zorzos et al. 2010). The second option is integrating neuron-sized LED sources mixed with the recording sites on the silicon probe (Kim et al. 2013). Either configuration will offer unmatched spatial precision and capability of targeted perturbation and recording from specified neuron types. Integration of optical stimulation, large-scale recording, and on-stage multiplexing will facilitate the dissemination and use such tools in a large number of laboratories for the investigation of multiple circuits and their behavior-dependent interactions in freely moving small rodents for testing hypotheses of neural networks and brain function.

\section{GRANTS}

This study was supported by National Institutes of Health Grants NS34994, MH-54671, and NS-074015, the Human Frontier Science Program, and the J. D. McDonnell Foundation. A. Berényi was supported by a Marie Curie FP7-PEOPLE-2009-IOF Grant (no. 254780), EU-FP7-ERC-2013-Starting
Grant (no. 337075), the Momentum Program of the Hungarian Academy of Sciences, and the Rosztóczy Foundation.

\section{DISCLOSURES}

A. Berényi is the founder of Amplipex Ltd., Szeged, Hungary, which manufactures signal-multiplexed headstages and demultiplexing systems. The other authors declare the absence of any commercial or financial relationships that could be construed as a potential conflict of interest.

\section{AUTHOR CONTRIBUTIONS}

A.B., A.L., T.D.H., and G.B. conception and design of research; A.B., A.J.N., L.R., J.D.L., S.F., and E.S. performed experiments; A.B., Z.S., L.R., S.F., E.S., and G.B. analyzed data; A.B., Z.S., L.R., J.D.L., S.F., E.S., and G.B. interpreted results of experiments; A.B., Z.S., and G.B. prepared figures; A.B. and G.B. drafted manuscript; A.B., Z.S., A.J.N., L.R., J.D.L., S.F., E.S., A.L., T.D.H., and G.B. edited and revised manuscript; A.B., Z.S., A.J.N., L.R., J.D.L., S.F., E.S., A.L., T.D.H., and G.B. approved final version of manuscript.

\section{REFERENCES}

Alivisatos AP, Chun M, Church GM, Deisseroth K, Donoghue JP, Greenspan RJ, McEuen PL, Roukes ML, Sejnowski TJ, Weiss PS, Yuste R. Neuroscience. The brain activity map. Science 339: 1284-1285, 2013.

Alivisatos AP, Chun M, Church GM, Greenspan RJ, Roukes ML, Yuste R. The brain activity map project and the challenge of functional connectomics. Neuron 74: 970-974, 2012

Anikeeva P, Andalman AS, Witten I, Warden M, Goshen I, Grosenick L, Gunaydin LA, Frank LM, Deisseroth K. Optetrode: a multichannel readout for optogenetic control in freely moving mice. Nat Neurosci 15: 163-170, 2012.

Barthó P, Hirase H, Monconduit L, Zugaro M, Harris KD, Buzsáki G. Characterization of neocortical principal cells and interneurons by network interactions and extracellular features. J Neurophysiol 92: 600-608, 2004.

Berényi A, Belluscio M, Mao D, Buzsáki G. Closed-loop control of epilepsy by transcranial electrical stimulation. Science 337: 735-737, 2012.

Blanche TJ, Spacek MA, Hetke JF, Swindale NV. Polytrodes: high-density silicon electrode arrays for large-scale multiunit recording. J Neurophysiol 93: 2987-3000, 2005.

Boyden ES, Zhang F, Bamberg E, Nagel G, Deisseroth K. Millisecondtimescale, genetically targeted optical control of neural activity. Nat Neurosci 8: 1263-1268, 2005.

Buzsáki G. Large-scale recording of neuronal ensembles. Nat Neurosci 7: 446-451, 2004.

Buzsáki G, Anastassiou CA, Koch C. The origin of extracellular fields and currents-EEG, ECoG, LFP and spikes. Nat Rev Neurosci 13: 407-420, 2012.

Carandini M. From circuits to behavior: a bridge too far? Nat Neurosci 15: 507-509, 2012.

Csicsvari J, Henze DA, Jamieson B, Harris KD, Sirota A, Barthó P, Wise KD, Buzsáki G. Massively parallel recording of unit and local field potentials with silicon-based electrodes. J Neurophysiol 90: 1314-1323, 2003

Du J, Blanche TJ, Harrison RR, Lester HA, Masmanidis SC. Multiplexed, high density electrophysiology with nanofabricated neural probes. PLoS One 6: e26204, 2011.

Du J, Riedel-Kruse IH, Nawroth JC, Roukes ML, Laurent G, Masmanidis SC. High-resolution three-dimensional extracellular recording of neuronal activity with microfabricated electrode arrays. J Neurophysiol 101: 16711678, 2009.

Fujisawa S, Amarasingham A, Harrison MT, Buzsáki G. Behavior-dependent short-term assembly dynamics in the medial prefrontal cortex. Nat Neurosci 11: 823-833, 2008.

Ghosh KK, Burns LD, Cocker ED, Nimmerjahn A, Ziv Y, Gamal AE, Schnitzer MJ. Miniaturized integration of a fluorescence microscope. Nat Methods 8: 871-878, 2011.

Greenwald E, Mollazadeh M, Hu C, Wei T, Culurciello E, Thakor V. A VLSI neural monitoring system with ultra-wideband telemetry for awake behaving subjects. IEEE Trans Biomed Circuits Syst 5: 112-119, 2011.

Harris KD, Henze DA, Csicsvari J, Hirase H, Buzsáki G. Accuracy of tetrode spike separation as determined by simultaneous intracellular and extracellular measurements. J Neurophysiol 84: 401-414, 2000. 
Harrison RR. The design of integrated circuits to observe brain activity. Proc IEEE 96: 1203-1216, 2008.

Harrison RR, Fotowat H, Chan R, Kier RJ, Olberg R, Leonardo A, Gabbiani F. Wireless neural/EMG telemetry systems for small freely moving animals. IEEE Trans Biomed Circuits Syst 5: 103-111, 2011.

Henze DA, Borhegyi Z, Csicsvari J, Mamiya A, Harris KD, Buzsáki G. Intracellular features predicted by extracellular recordings in the hippocampus in vivo. J Neurophysiol 84: 390-400, 2000.

Hirabayashi T, Takeuchi D, Tamura K, Miyashita Y. Microcircuits for hierarchical elaboration of object coding across primate temporal areas. Science 341: 191-195, 2013.

Kim TI, McCall JG, Jung YH, Huang X, Siuda ER, Li Y, Song J, Song YM, Pao HA, Kim RH, Lu C, Lee SD, Song IS, Shin G, Al-Hasani R, Kim S, Tan MP, Huang Y, Omenetto FG, Rogers JA, Bruchas MR. Injectable, cellular-scale optoelectronics with applications for wireless optogenetics. Science 340: 211-216, 2013.

Kipke DR, Shain W, Buzsáki G, Fetz E, Henderson JM, Hetke JF, Schalk G. Advanced neurotechnologies for chronic neural interfaces: new horizons and clinical opportunities. J Neurosci 28: 11830-11838, 2008.

Lein ES, Hawrylycz MJ, Ao N, Ayres M, Bensinger A, Bernard A, Boe AF, Boguski MS, Brockway KS, Byrnes EJ, Chen L, Chen L, Chen TM, Chin MC, Chong J, Crook BE, Czaplinska A, Dang CN, Datta S, Dee NR, Desaki AL, Desta T, Diep E, Dolbeare TA, Donelan MJ, Dong HW, Dougherty JG, Duncan BJ, Ebbert AJ, Eichele G, Estin LK, Faber C, Facer BA, Fields R, Fischer SR, Fliss TP, Frensley C, Gates SN, Glattfelder KJ, Halverson KR, Hart MR, Hohmann JG, Howell MP, Jeung DP, Johnson RA, Karr PT, Kawal R, Kidney JM, Knapik RH, Kuan CL, Lake JH, Laramee AR, Larsen KD, Lau C, Lemon TA, Liang AJ, Liu Y, Luong LT, Michaels J, Morgan JJ, Morgan RJ, Mortrud MT, Mosqueda NF, Ng LL, Ng R, Orta GJ, Overly CC, Pak TH, Parry SE, Pathak SD, Pearson OC, Puchalski RB, Riley ZL, Rockett HR, Rowland SA, Royall JJ, Ruiz MJ, Sarno NR, Schaffnit K, Shapovalova NV, Sivisay T, Slaughterbeck CR, Smith SC, Smith KA, Smith BI, Sodt AJ, Stewart NN, Stumpf KR, Sunkin SM, Sutram M, Tam A, Teemer CD, Thaller C, Thompson CL, Varnam LR, Visel A, Whitlock RM, Wohnoutka PE, Wolkey CK, Wong VY, Wood M, Yaylaoglu MB, Young RC, Youngstrom BL, Yuan XF, Zhang B, Zwingman TA, Jones AR. Genome-wide atlas of gene expression in the adult mouse brain. Nature 445: 168-176, 2007.

Lichtman JW, Denk W. The big and the small: challenges of imaging the brain's circuits. Science 334: 618-623, 2011.

Logothetis NK. The underpinnings of the BOLD functional magnetic resonance imaging signal. J Neurosci 23: 3963-3971, 2003.

Madisen L, Mao T, Koch H, Zhuo JM, Berenyi A, Fujisawa S, Hsu YW, Garcia AJ 3rd, Gu X, Zanella S, Kidney J, Gu H, Mao Y, Hooks BM, Boyden ES, Buzsáki G, Ramirez JM, Jones AR, Svoboda K, Han X, Turner EE, Zeng H. A toolbox of Cre-dependent optogenetic transgenic mice for light-induced activation and silencing. Nat Neurosci 15: 793-802, 2012.

Mizuseki K, Sirota A, Pastalkova E, Buzsáki G. Theta oscillations provide temporal windows for local circuit computation in the entorhinal-hippocampal loop. Neuron 64: 267-280, 2009.

Montgomery SM, Sirota A, Buzsáki G. Theta and gamma coordination of hippocampal networks during waking and rapid eye movement sleep. $J$ Neurosci 28: 6731-6741, 2008.

Moser EI, Kropff E, Moser MB. Place cells, grid cells, and the brain's spatial representation system. Anпи Rev Neurosci 31: 69-89, 2008.

Nicolelis MA, Ghazanfar AA, Faggin BM, Votaw S, Oliveira LM. Reconstructing the engram: simultaneous, multisite, many single neuron recordings. Neuron 18: 529-537, 1997.

O'Keefe J, Nadel L. The Hippocampus as a Cognitive Map. Oxford, UK: Clarendon Press, 1978.

Olsson RH 3rd, Buhl DL, Sirota AM, Buzsaki G, Wise KD. Band-tunable and multiplexed integrated circuits for simultaneous recording and stimula- tion with microelectrode arrays. IEEE Trans Biomed Eng 52: 1303-1311, 2005.

Prakash R, Yizhar O, Grewe B, Ramakrishnan C, Wang N, Goshen I, Packer AM, Peterka DS, Yuste R, Schnitzer MJ, Deisseroth K. Twophoton optogenetic toolbox for fast inhibition, excitation and bistable modulation. Nat Methods 9: 1171-1179, 2012.

Ray S, Maunsell JH. Different origins of gamma rhythm and high-gamma activity in macaque visual cortex. PLoS Biol 9: e1000610, 2011.

Royer S, Zemelman BV, Barbic M, Losonczy A, Buzsáki G, Magee JC. Multi-array silicon probes with integrated optical fibers: light-assisted perturbation and recording of local neural circuits in the behaving animal. Eur J Neurosci 31: 2279-2291, 2010.

Royer S, Zemelman BV, Losonczy A, Kim J, Chance F, Magee JC, Buzsáki G. Control of timing, rate and bursts of hippocampal place cells by dendritic and somatic inhibition. Nat Neurosci 15: 769-775, 2012.

Sirota A, Montgomery S, Fujisawa S, Isomura Y, Zugaro M, Buzsáki G. Entrainment of neocortical neurons and gamma oscillations by the hippocampal theta rhythm. Neuron 60: 683-697, 2008.

Sodagar AM, Wise KD, Najafi K. A fully integrated mixed-signal neural processor for implantable multichannel cortical recording. IEEE Trans Biomed Eng 54: 1075-1088, 2007.

Stark E, Koos T, Buzsáki G. Diode probes for spatiotemporal optical control of multiple neurons in freely moving animals. J Neurophysiol 108: 349 363, 2012.

Szuts TA, Fadeyev V, Kachiguine S, Sher A, Grivich MV, Agrochao M, Hottowy P, Dabrowski W, Lubenov EV, Siapas AG, Uchida N, Litke AM, Meister M. A wireless multi-channel neural amplifier for freely moving animals. Nat Neurosci 14: 263-269, 2011.

Taniguchi H, He M, Wu P, Kim S, Paik R, Sugino K, Kvitsiani D, Fu Y, Lu J, Lin Y, Miyoshi G, Shima Y, Fishell G, Nelson SB, Huang ZJ. A resource of Cre driver lines for genetic targeting of GABAergic neurons in cerebral cortex. Neuron 71: 995-1013, 2011.

Tye KM, Deisseroth K. Optogenetic investigation of neural circuits underlying brain disease in animal models. Nat Rev Neurosci 13: 251-266, 2012.

Vandecasteele M, M S, Royer S, Belluscio M, Berényi A, Diba K, Fujisawa S, Grosmark A, Mao D, Mizuseki K, Patel J, Stark E, Sullivan D, Watson B, Buzsáki G. Large-scale recording of neurons by movable silicon probes in behaving rodents. $J$ Vis Exp 61: e3568, 2012.

Viventi J, Kim DH, Vigeland L, Frechette ES, Blanco JA, Kim YS, Avrin AE, Tiruvadi VR, Hwang SW, Vanleer AC, Wulsin DF, Davis K, Gelber CE, Palmer L, Van der Spiegel J, Wu J, Xiao J, Huang Y, Contreras D, Rogers JA, Litt B. Flexible, foldable, actively multiplexed, high-density electrode array for mapping brain activity in vivo. Nat Neurosci 14: 1599-1605, 2011.

Wilson MA, McNaughton BL. Dynamics of the hippocampal ensemble code for space. Science 261: 1055-1058, 1993.

Wise KD, Anderson DJ, Hetke JF, Kipke DR, Najafi K. Wireless implantable microsystems: high-density electronic interfaces to the nervous system. Proc IEEE 92: 76-97, 2004.

Wu F, Stark E, Im M, Cho IJ, Yoon ES, Buzsáki G, Wise KD, Yoon E. An implantable neural probe with monolithically integrated dielectric waveguide and recording electrodes for optogenetics applications. J Neural Eng 10: 056012, 2013.

Yartsev MM, Ulanovsky N. Representation of three-dimensional space in the hippocampus of flying bats. Science 340: 367-372, 2013.

Yizhar O, Fenno LE, Davidson TJ, Mogri M, Deisseroth K. Optogenetics in neural systems. Neuron 71: 9-34, 2011.

Ylinen A, Bragin A, Nádasdy Z, Jandó G, Szabó I, Sik A, Buzsáki G. Sharp wave-associated high-frequency oscillation $(200 \mathrm{~Hz})$ in the intact hippocampus: network and intracellular mechanisms. J Neurosci 15: 30-46, 1995.

Zorzos AN, Boyden ES, Fonstad CG. Multiwaveguide implantable probe for light delivery to sets of distributed brain targets. Opt Lett 35: 4133-4135, 2010 